\title{
EVOLUTION IN THE GENUS RHINELLA: A TOTAL EVIDENCE PHYLOGENETIC ANALYSIS OF NEOTROPICAL TRUE TOADS (ANURA: BUFONIDAE)
}

MARTÍN O. PEREYRA, BORIS L. BLOTTO, DIEGO BALDO, JUAN C. CHAPARRO, SANTIAGO R. RON, AGUSTÍN J. ELIAS-COSTA, PATRICIA P. IGLESIAS, PABLO J. VENEGAS, MARIA TEREZA C. THOMÉ, JHON JAIRO OSPINA-SARRIA, NATAN M. MACIEL, MARCO RADA, FRANCISCO KOLENC, CLAUDIO BORTEIRO, MAURICIO RIVERA-CORREA, FERNANDO J.M. ROJAS-RUNJAIC, JIŘÍ MORAVEC, IGNACIO DE LA RIVA, WARD C. WHEELER, SANTIAGO CASTROVIEJO-FISHER, TARAN GRANT, CÉLIO F.B. HADDAD, AND JULIÁN FAIVOVICH

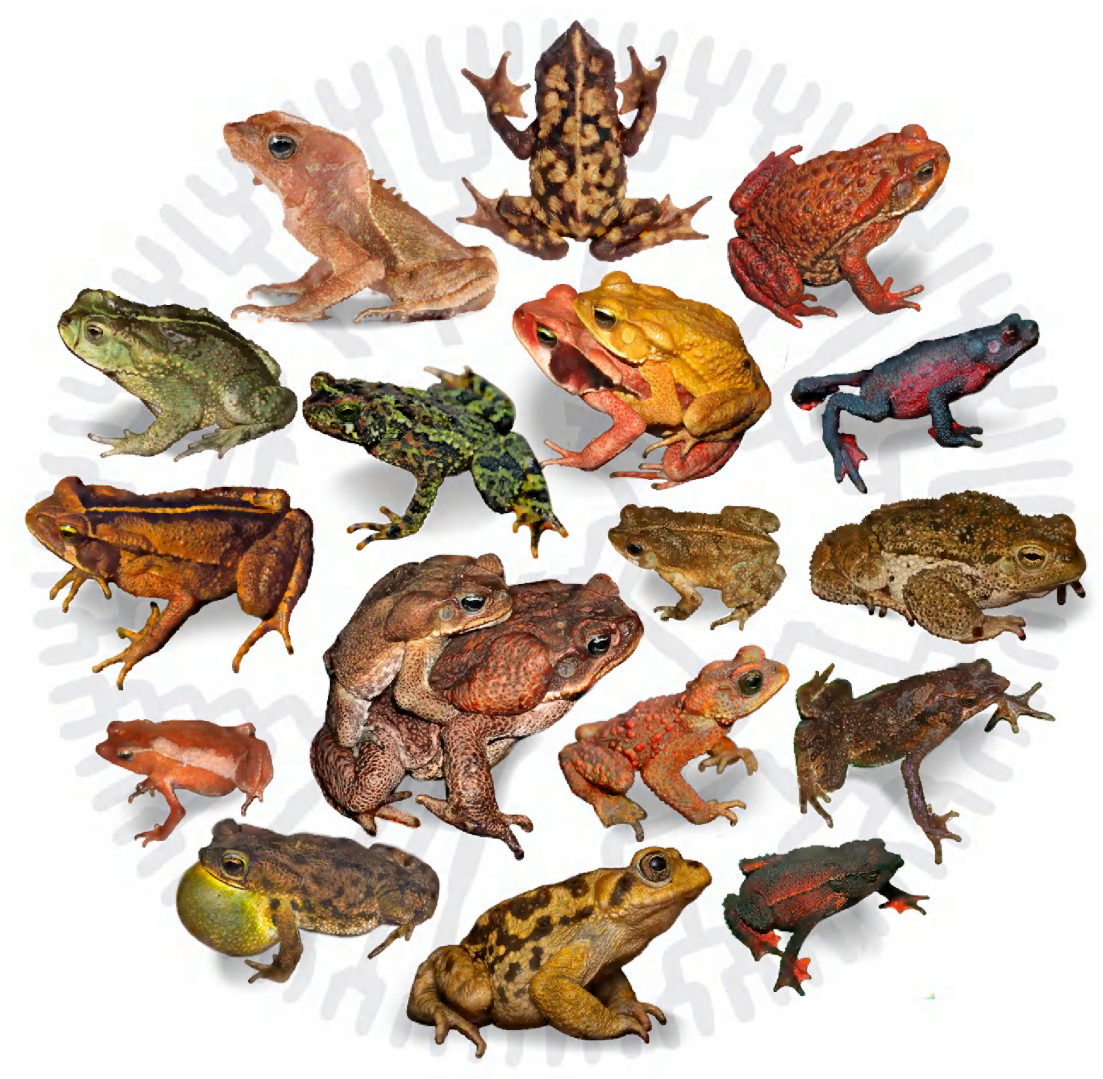

BULLETIN OF THE AMERICAN MUSEUM OF NATURAL HISTORY 


\section{EVOLUTION IN THE GENUS RHINELLA: A TOTAL EVIDENCE PHYLOGENETIC ANALYSIS OF NEOTROPICAL TRUE TOADS (ANURA: BUFONIDAE)}

MARTÍN O. PEREYRA, BORIS L. BLOTTO, DIEGO BALDO, JUAN C. CHAPARRO, SANTIAGO R. RON, AGUSTÍN J. ELIAS-COSTA, PATRICIA P. IGLESIAS, PABLO J. VENEGAS, MARIA TEREZA C. THOMÉ, JHON JAIRO OSPINA-SARRIA, NATAN M. MACIEL, MARCO RADA, FRANCISCO KOLENC, CLAUDIO BORTEIRO, MAURICIO RIVERA-CORREA, FERNANDO J.M. ROJAS-RUNJAIC, JIŘÍ MORAVEC, IGNACIO DE LA RIVA, WARD C. WHEELER, SANTIAGO CASTROVIEJO-FISHER, TARAN GRANT, CÉLIO F.B. HADDAD, JULIÁN FAIVOVICH

BULLETIN OF THE AMERICAN MUSEUM OF NATURAL HISTORY

Number 447, 155 pp., 15 figures, 11 tables

Issued March 31, 2021 


\section{CONTENTS}

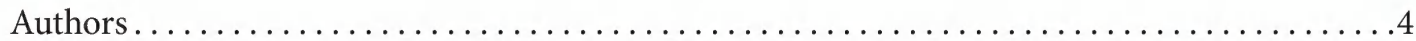

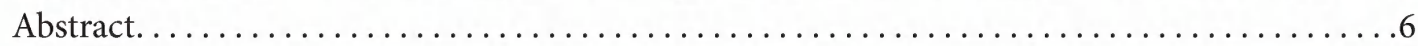

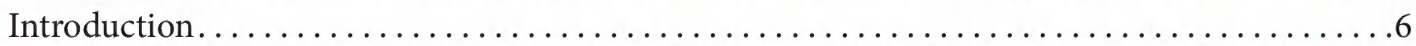

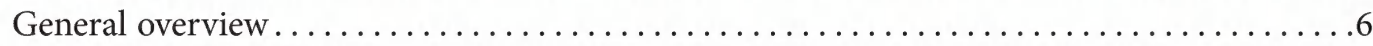

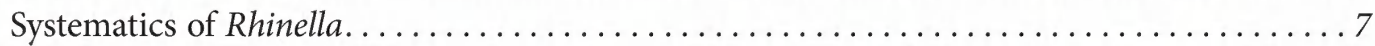

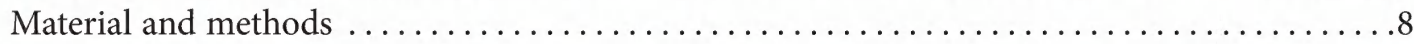

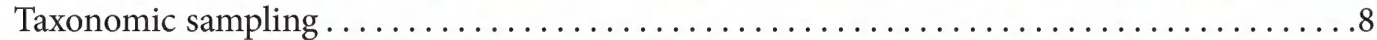

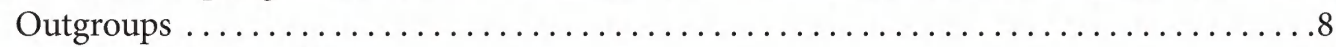

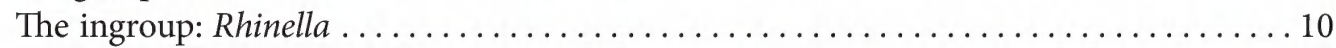

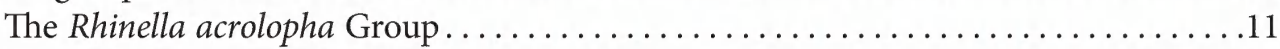

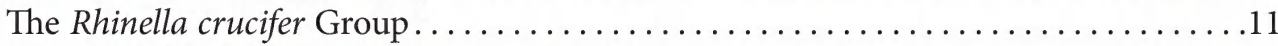

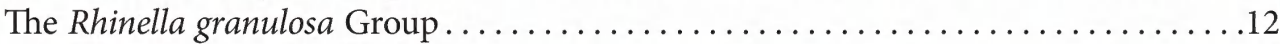

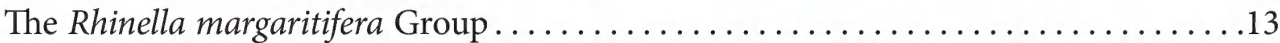

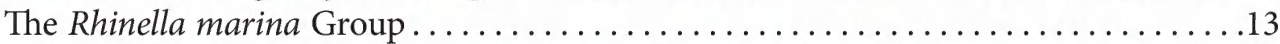

The Rhinella spinulosa Group .........................................

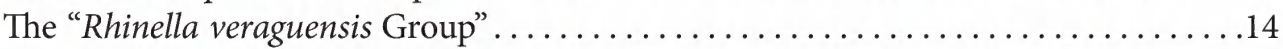

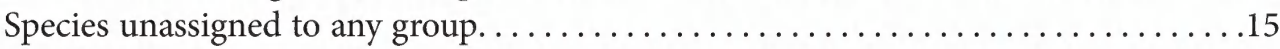

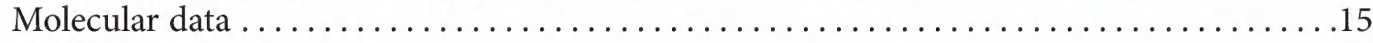

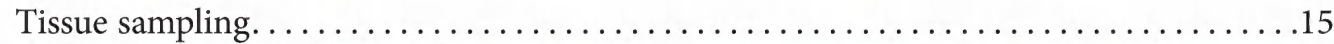

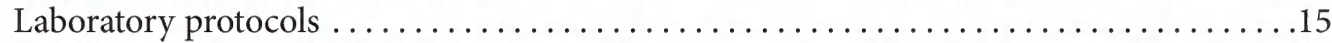

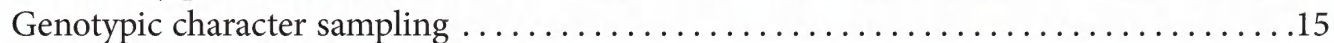

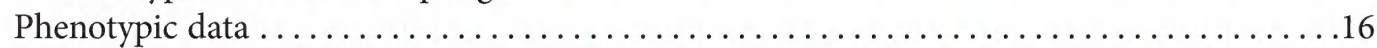

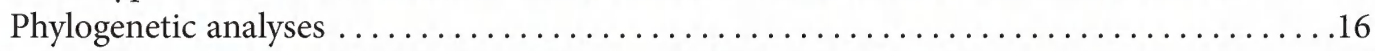

Separate phylogenetic analyses of nuclear and mitochondrial sequences............17

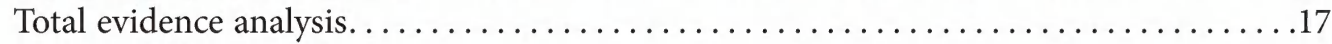

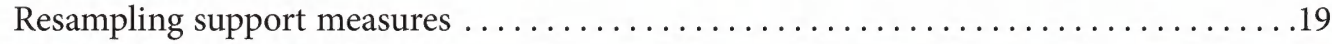

Maximum likelihood analysis. ....................................

Taxonomic evaluation . . . . . . . . . . . . . . . . . . . . . . . . . . . . . . . . 19

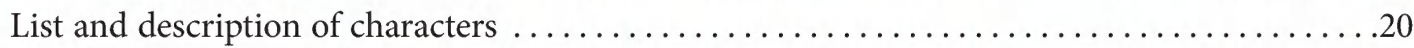

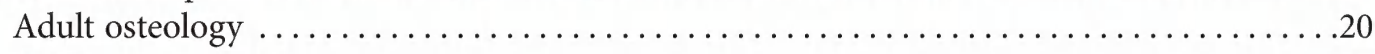

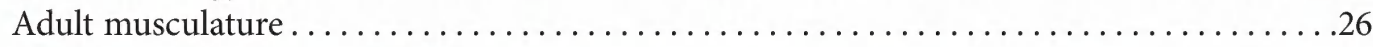

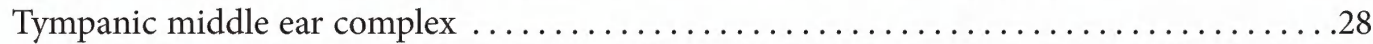

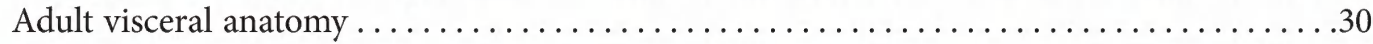

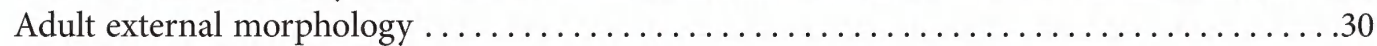

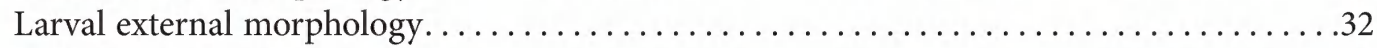

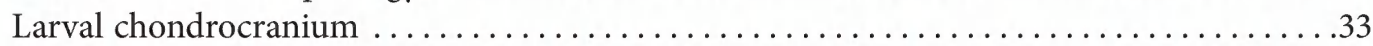

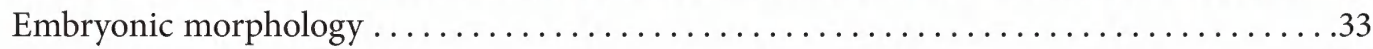

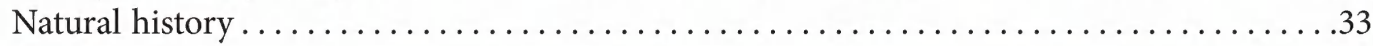

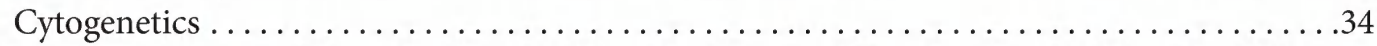

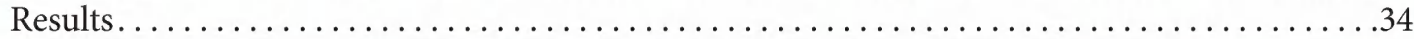

Separate analyses of restricted nuclear (rND) and mitochondrial (rMD) datasets .......34

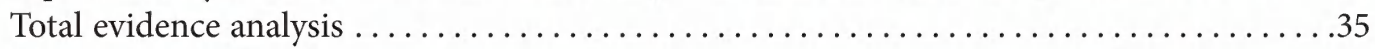

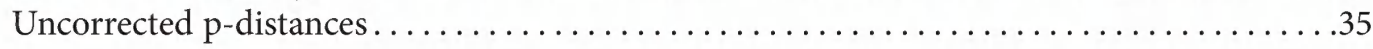




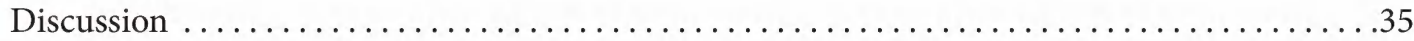

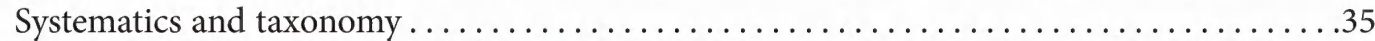

Relationships among outgroups and Rhinella ........................ 35

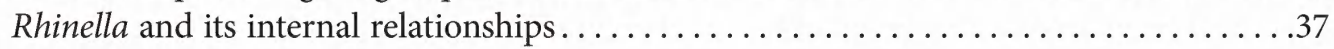

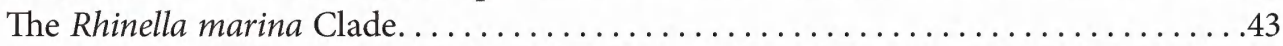

The Rhinella arunco Group....................................... 44

The Rhinella spinulosa Group.................................45

The Rhinella granulosa Group .................................47

The mitochondrial lineage of Rhinella horribilis................... 51

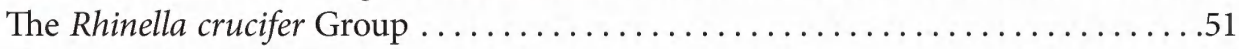

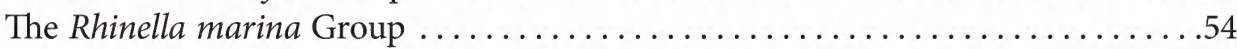

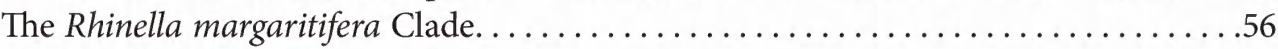

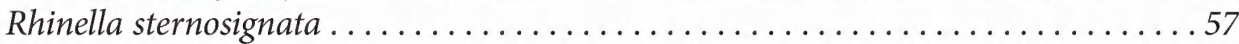

The Rhinella veraguensis Group...............................57

The Rhinella festae Group .................................60

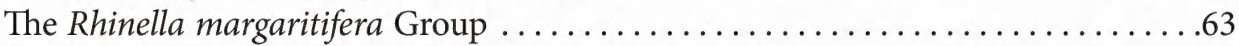

Hybridization, deep mitochondrial divergence, and "ghost introgression" in Rhinella. . . . 69

Comments on the phenotypic evidence considered for Rhinella ............... 73

The fossil record of Rhinella and calibration points.......................... 73

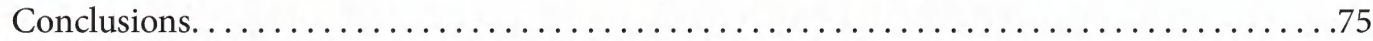

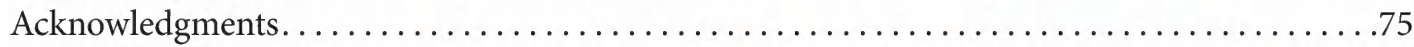

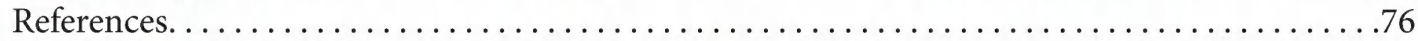

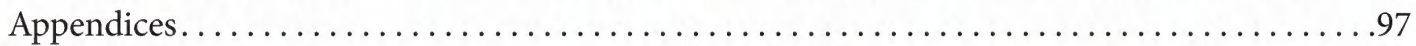

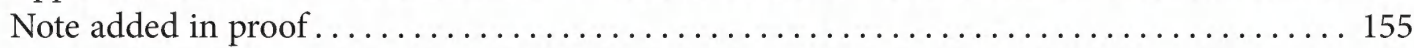




\section{AUTHORS}

Martín O. Pereyra: División Herpetología, Museo Argentino de Ciencias Naturales "Bernardino Rivadavia"-CONICET, Buenos Aires; and Laboratorio de Genética Evolutiva "Claudio J. Bidau," Instituto de Biología Subtropical (IBS, CONICET), Universidad Nacional de Misiones (UNaM), Posadas, Misiones, Argentina.

Boris L. Blotto: División Herpetología, Museo Argentino de Ciencias Naturales "Bernardino Rivadavia"-CONICET, Buenos Aires; Universidade Estadual Paulista (UNESP), Instituto de Biociências, Departamento de Biodiversidade e Centro de Aquicultura (CAUNESP), Rio Claro, São Paulo; and Departamento de Zoologia, Instituto de Biociências, Universidade de São Paulo, São Paulo.

Diego BAldo: Laboratorio de Genética Evolutiva “Claudio J. Bidau," Instituto de Biología Subtropical (IBS, CONICET), Universidad Nacional de Misiones (UNaM), Posadas, Misiones, Argentina.

Juan C. Chaparro: Museo de Biodiversidad del Perú, Cusco, Perú; and Museo de Historia Natural de la Universidad Nacional de San Antonio Abad del Cusco, Paraninfo Universitario, Cusco.

Santiago R. Ron: Museo de Zoología, Escuela de Biología, Pontificia Universidad Católica del Ecuador, Quito.

Agustín J. Elias-Costa: División Herpetología, Museo Argentino de Ciencias Naturales "Bernardino Rivadavia"-CONICET, Buenos Aires.

Patricia P. Iglesias: Laboratorio de Genética Evolutiva “Claudio J. Bidau”, Instituto de Biología Subtropical (IBS, CONICET), Universidad Nacional de Misiones (UNaM), Posadas, Misiones, Argentina.

Pablo J. Venegas: División de Herpetología-Centro de Ornitología y Biodiversidad (CORBIDI), Surco, Lima.

Maria Tereza C. Thomé: Universidade Estadual Paulista (UNESP), Instituto de Biociências, Departamento de Biodiversidade e Centro de Aquicultura (CAUNESP), Rio Claro, São Paulo.

Jhon Jairo Ospina-Sarria: Departamento de Zoologia, Instituto de Biociências, Universidade de São Paulo, São Paulo, Brazil; and Calima, Fundación para la Investigación de la Biodiversidad y Conservación en el Trópico, Cali.

Natan M. Maciel: Laboratório de Herpetologia e Comportamento Animal, Departamento de Ecologia, Instituto de Ciências Biológicas, Universidade Federal de Goiás, Goiânia, Brazil.

Marco RAda: Departamento de Zoologia, Instituto de Biociências, Universidade de São Paulo, São Paulo.

Francisco Kolenc: Sección Herpetología, Museo Nacional de Historia Natural, Montevideo.

Claudio Borteiro: Sección Herpetología, Museo Nacional de Historia Natural, Montevideo.

Mauricio Rivera-Correa: Grupo Herpetológico de Antioquia, Instituto de Biología, Universidad de Antioquia, Medellín. 
Fernando J.M. Rojas-Runjaic: Fundación La Salle de Ciencias Naturales, Museo de Historia Natural La Salle (MHNLS), Venezuela; and Pontifícia Universidade Católica do Rio Grande do Sul (PUCRS), Porto Alegre, Rio Grande do Sul, Brazil.

Jiří Moravec: Department of Zoology, National Museum, Prague, Czech Republic.

IgnaCio de la Riva: Museo Nacional de Ciencias Naturales (MNCN-CSIC), Madrid.

WARD C. WheELER: Division of Invertebrate Zoology, American Museum of Natural History, New York.

Santiago Castroviejo-Fisher: Pontifícia Universidade Católica do Rio Grande do Sul (PUCRS), Porto Alegre, Rio Grande do Sul, Brazil; and Research Associate, Herpetology, Division of Vertebrate Zoology, American Museum of Natural History, New York.

Taran Grant: Departamento de Zoologia, Instituto de Biociências, Universidade de São Paulo, São Paulo; and Research Associate, Herpetology, Division of Vertebrate Zoology, American Museum of Natural History, New York.

CÉLio F.B. Haddad: Universidade Estadual Paulista (UNESP), Instituto de Biociências, Departamento de Biodiversidade e Centro de Aquicultura (CAUNESP), Rio Claro, São Paulo.

Julián Faivovich: División Herpetología, Museo Argentino de Ciencias Naturales "Bernardino Rivadavia"-CONICET, Buenos Aires; Departamento de Biodiversidad y Biología Experimental, Facultad de Ciencias Exactas y Naturales, Universidad de Buenos Aires, Buenos Aires; and Research Associate, Herpetology, Division of Vertebrate Zoology, American Museum of Natural History, New York.

This publication has been registered in ZooBank (urn:lsid:zoobank.org:pub:56ABAF36-1B0A-4AE3-A3B4-1283545A675F). 


\begin{abstract}
True toads of the genus Rhinella are among the most common and diverse group of Neotropical anurans. These toads are widely distributed throughout South America, inhabiting a great diversity of environments and ecoregions. Currently, however, the genus is defined solely on the basis of molecular characters, and it lacks a proper diagnosis. Although some phenetic species groups have traditionally been recognized within Rhinella, the monophyly of some of them have been rejected in previous phylogenetic analyses, and many species remain unassigned to these poorly defined groups. Additionally, the identity and taxonomy of several species are problematic and hinder the specific recognition and description of undescribed taxa. In this work, we first perform phylogenetic analyses of separate mitochondrial and nuclear datasets to test the possible occurrence of hybridization and/or genetic introgression in the genus. The comparative analysis of both datasets revealed unidirectional mitochondrial introgressions of an unknown parental species into $R$. horribilis ("ghost introgression") and of R. dorbignyi into R. bernardoi; therefore, the mitochondrial and nuclear datasets of these species were considered separately in subsequent analyses. We performed total-evidence phylogenetic analyses that included revised molecular (four mitochondrial and five nuclear genes) and phenotypic (90 characters) datasets for 83 nominal species of Rhinella, plus several undescribed and problematic species and multiple outgroups. Results demonstrate that Rhinella was nonmonophyletic due to the position of $R$. ceratophrys, which was recovered as the sister taxon of Rhaebo nasicus with strong support. Among our outgroups, the strongly supported Anaxyrus + Incilius is the sister clade of all other species of Rhinella. Once R. ceratophrys is excluded, the genus Rhinella is monophyletic, well supported, and composed of two major clades. One of these is moderately supported and includes species of the former R. spinulosa Group (including R. gallardoi); the monophyletic R. granulosa, R. crucifer, and R. marina Groups; and a clade composed of the mitochondrial sequences of $R$. horribilis. The other major clade is strongly supported and composed of all the species from the non-monophyletic $R$. veraguensis and $R$. margaritifera Groups, the former $R$. acrolopha Group, and $R$. sternosignata. Consistent with these results, we define eight species groups of Rhinella that are mostly diagnosed by phenotypic synapomorphies in addition to a combination of morphological character states. Rhinella sternosignata is the only species that remains unassigned to any group. We also synonymize nine species, treat three former subspecies as full species, and suggest that 15 lineages represent putative undescribed species. Lastly, we discuss the apparently frequent occurrence of hybridization, deep mitochondrial divergence, and "ghost introgression"; the incomplete phenotypic evidence (including putative character systems that could be used for future phylogenetic analyses); and the validity of the known fossil record of Rhinella as a source of calibration points for divergence dating analyses.
\end{abstract}

\section{INTRODUCTION}

\section{General Overview}

True toads of the former genus Bufo are a popular group of anurans distributed nearly worldwide, and widely studied by researchers from different disciplines. The classic book "Evolution in the genus Bufo" (Blair, 1972) synthesized knowledge about the morphology, phylogeny, and biology of the group. Despite having integrated evidence from many sources of characters to elucidate the evolutionary relationships among the species groups of true toads, this work largely revealed the difficulties to study their phylogenetic relationships. It was not until the 1990 s-2000s that a general picture of these relationships emerged, and the taxonomy of true toads was revised to be consistent with phylogenetic hypotheses (Graybeal, 1997; Pauly et al., 2004; Frost et al., 2006; Pramuk, 2006). Currently, most of the South American true toads of the former genus Bufo are grouped in the large genus Rhinella (Chaparro et al., 2007). 
Rhinella includes many of the most conspicuous and ubiquitous species of the anuran fauna in almost all the major biogeographic areas of the Neotropical region (Duellman, 1999; Frost, 2020; IUCN, 2020). With 92 species, Rhinella is the second largest genus of Bufonidae, and its species show considerable morphological and biological diversity, including large variation in size, different levels of cranial ossification, integumentary structure, larval morphology, and ecological and reproductive diversity characteristics (Trueb, 1971; Cei, 1972a; Toledo and Jared, 1993; Pramuk, 2006; Aguayo et al., 2009; van Bocxlaer et al., 2010; Pereyra et al., 2015; Bandeira et al., 2016; Simon et al., 2016; Hudson et al., 2018). Some common species of Rhinella (e.g., R. arenarum, $R$. horribilis, and $R$. marina) have been employed extensively as model organisms for various biological disciplines, such as biochemistry (e.g., Abel and Macht, 1912; Cei et al., 1968; Rash et al., 2011), developmental biology (e.g., Markovich and Regeer, 1999; Barisone et al., 2002; Brown et al., 2002), ecotoxicology (e.g., Lajmanovich et al., 2011), molecular biology (e.g., Estoup et al., 2004, 2010; Rollins et al., 2015; Edwards et al., 2018; Ceschin et al., 2020), and especially physiology (e.g., Houssay and Giusti, 1929; Houssay, 1949; Penhos et al., 1967; Sassone et al., 2015). This genus also contains a highly invasive species, $R$. marina, widely introduced into many countries and islands from different continents (Frost, 2020), where usually it has a highly negative ecological and socioeconomic impact (Jolly et al., 2015; Bacher et al., 2018).

\section{Systematics of RHINELLA}

For decades, all South American true toads were part of the formerly large and poorly defined genus Bufo, which included a heterogeneous group of toads distributed throughout Africa, America, and Eurasia (e.g., Blair, 1972; Graybeal, 1997). Frost et al. (2006) partitioned this polyphyletic genus into monophyletic units mostly on the basis of the results of their phylogenetic analysis but also on the results of previous studies (e.g., Graybeal, 1997; Pauly et al., 2004). Frost et al.
(2006) resurrected Rhinella for the species of the former Bufo margaritifer Group, which they recovered as distantly related to the other species of South American true toads included in their analysis, including Chaunus and Rhaebo (both also resurrected by Frost et al., 2006). Frost et al. (2006) noted that Bufo margaritifer was nested within Chaunus in a previous phylogenetic study (Pauly et al., 2004), a finding that was subsequently supported by Pramuk (2006) and Chaparro et al. (2007). Therefore, Rhinella was later redefined to include the species of Chaunus and Rhamphophryne as well (Chaparro et al., 2007).

The species groups of the former Bufo now referred to Rhinella were all recognized primarily on the basis of osteological characters and external morphology that were interpreted without quantitative phylogenetic analyses (Tihen, 1962; Cei, 1972a; R.F. Martin, 1972a, 1972b; Duellman and Schulte, 1992), including the R. crucifer, R. granulosa, $R$. margaritifera, $R$. marina, $R$. spinulosa, and $R$. veraguensis Groups. Pramuk (2006) studied the phylogenetic relationships of these toads on the basis of a combined analysis of morphological (mostly osteological) and molecular evidence. She rejected the monophyly of some of these species groups (e.g., the R. veraguensis Group is polyphyletic with respect to $R$. ocellata, the $R$. margaritifera Group, and Rhamphophryne), but did not modify their composition or diagnosis.

The subsequent increase in the knowledge of relations within Rhinella was limited to the addition of available sequences of some species in extensive phylogenetic analyses of Bufonidae or Anura (e.g., van Bocxlaer et al., 2010; Pyron and Wiens, 2011; Pyron, 2014; Jetz and Pyron, 2018). Figure 1 summarizes the main results of the more inclusive analyses of Rhinella.

For well over a decade, the systematics of Rhinella as a whole has languished, although several efforts focusing on the relationships and taxonomy of parts of the genus have been undertaken. These include phylogenetic analyses of presumptively monophyletic species groups (i.e., the $R$. crucifer, R. granulosa, and R. marina Groups; Maciel et al., 2006, 2010; Thomé et al., 2010, 2012; 
Vallinoto et al., 2010; Pereyra et al., 2016a) or fractions of the diversity of certain groups (i.e., the $R$. festae and $R$. margaritifera Groups; Fouquet et al., 2007a; Moravec et al., 2014; Santos et al., 2015; Cusi et al., 2017; Avila et al., 2018). Most recent studies on Rhinella aimed primarily to resolve species-level taxonomic problems (e.g., Fouquet et al., 2007a; Narvaes and Rodrigues, 2009; Jansen et al., 2011; Grant and Bolívar-G., 2014; Moravec et al., 2014; Cusi et al., 2017). Consequently, more than a decade after Pramuk's (2006) revision, species groups remain poorly defined, several species cannot be assigned to any of them, and few additional phenotypic synapomorphies have been proposed for Rhinella or its internal clades (Hoogmoed, 1986; 1990; La Marca and MijaresUrrutia, 1996; Pramuk, 2006; Chaparro et al., 2007; Padial et al., 2009; Blotto et al., 2014; Grant and Bolívar-G., 2014; Pereyra et al., 2016a).

Natural hybridization is common in several groups of Bufonidae, including many species of Rhinella (Blair, 1972; Feder, 1979; Haddad et al., 1990; Masta et al., 2002; Azevedo et al., 2003; Green and Parent, 2003; Yamazaki et al., 2008; Fontenot et al., 2011; Guerra et al., 2011), and mitochondrial and nuclear introgression have been corroborated in some of these clades (e.g. Green and Parent, 2003; Yamazaki et al., 2008; Fontenot et al., 2011; Dufresnes et al., 2019). Pereyra et al. (2016a) demonstrated the occurrence of hybridization events in the $R$. granulosa Group and unidirectional mitochondrial introgression of $R$. dorbignyi into $R$. bernardoi. A similar situation might exist between $R$. marina and $R$. diptycha, although the evidence is not conclusive (Sequeira et al., 2011; Vallinoto et al., 2017). The impact of these phenomena on the inference of phylogenetic relationships (Hennig, 1966; McDade, 1992; Posada and Crandall, 2002) could be mitigated, at least partially, if detected. A detailed evaluation of the discordance between mitochondrial and nuclear genomes together with a critical taxonomic evaluation provide an effective way to detect hybridization/introgression (Pereyra et al., 2016a).

In this paper, we present a densely sampled phylogenetic analysis of Rhinella, including 83 of its 92 species, using molecular (four mitochondrial and five nuclear genes) and phenotypic characters (90 characters from multiple character systems). The goals of this study are to (1) perform a stringent test of the monophyly of Rhinella as well as similar tests on all its species groups, (2) identify phenotypic synapomorphies to diagnose the species groups of Rhinella, and (3) to evaluate the taxonomic status of several taxa.

\section{MATERIAL AND METHODS}

\section{TAXONOMIC SAMPLING}

For the complete dataset (molecular and phenotypic), we sampled 83 described species of Rhinella (including all but nine of the currently recognized species), and 36 exemplar species of other bufonid genera as outgroups (see below). The outgroup species were chosen to provide a severe test of the monophyly of Rhinella, whereas the dense sampling within Rhinella allowed us to rigorously test the monophyly of all its species groups. All specimens scored for phenotypic data were associated with the most morphologically similar and/or geographically closest conspecific terminal of the molecular dataset for the total evidence (TE) analysis.

Collection and locality data of vouchers for sequences used in this study, including the information of the sources of the sequences (this work or previous studies), are detailed in appendix 1, and GenBank accession numbers are listed in appendix 2. A list of the species, specimens, and bibliography analyzed for character scoring of the phenotypic dataset is given in appendix 3, and the collection and locality data of specimens studied for morphology are provided in appendix 4.

\section{OUtGROUPS}

For outgroup sampling, we considered the results of the most recent phylogenetic analyses (Frost et al., 2006; Pramuk, 2006; van Bocxlaer et 


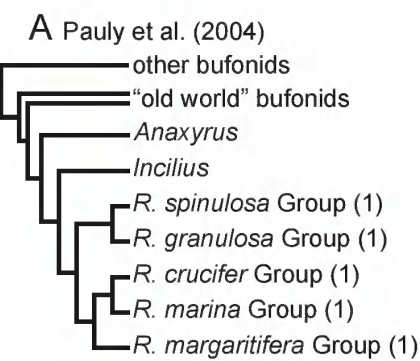

D Chaparro et al. (2007)

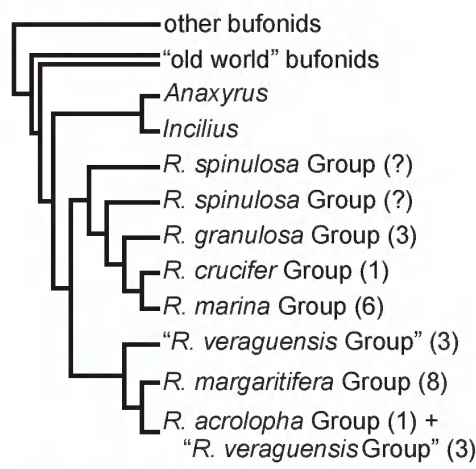

G Pyron (2014)

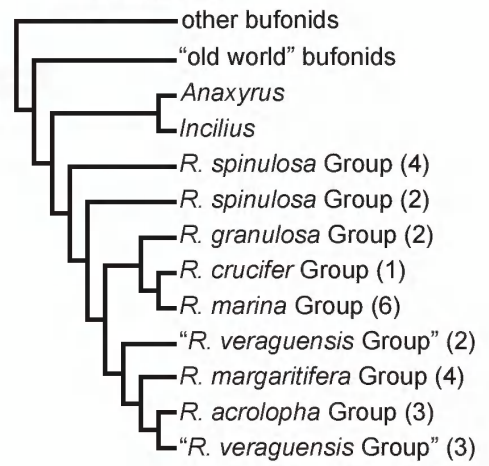

B Frost et al. (2006)

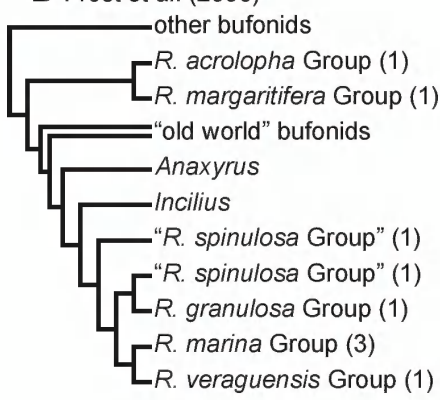

E van Bocxlaer et al. (2010)

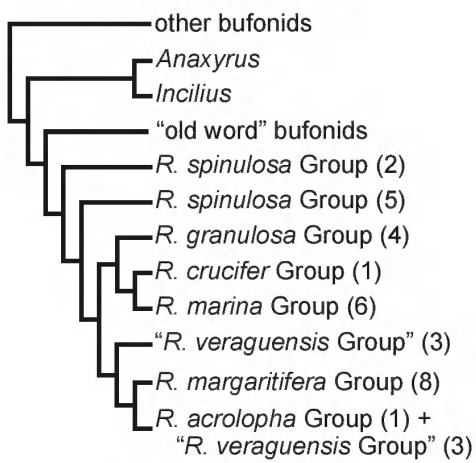

H Pereyra et al. (2016a)

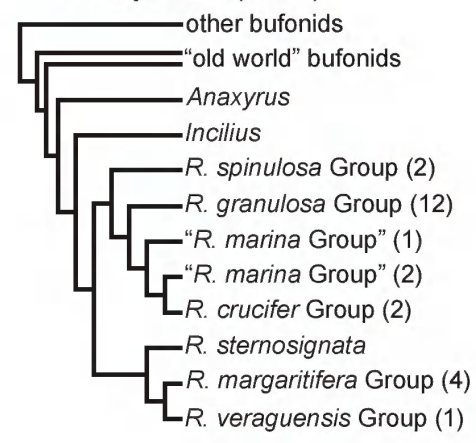

C Pramuk (2006)

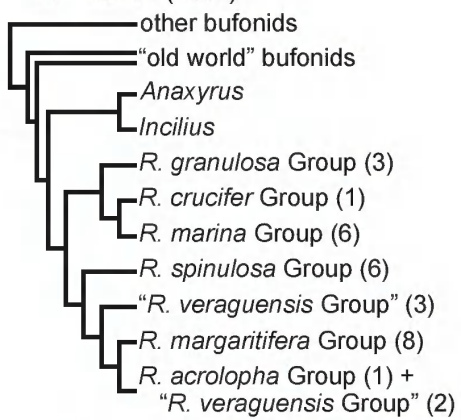

F Pyron and Wiens (2011)

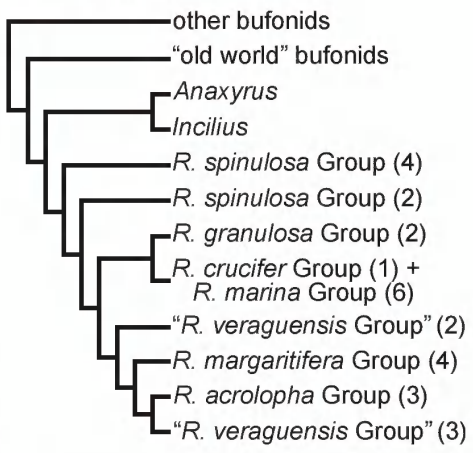

I Jetz and Pyron (2018)

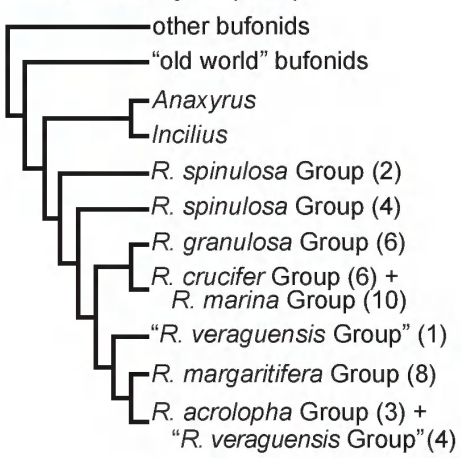

FIG. 1. Summarized relationships of Rhinella according to the main published phylogenetic hypotheses of the group. Only the topological sections corresponding to Rhinella, and putative most related outgroups (i.e., Anaxyrus and Incilius) are shown. The number of species sampled within each clade is reported in parentheses. (A) Pauly et al. (2004: fig. 2). (B) Frost et al. (2006: fig. 50). (C) Pramuk (2006: fig. 4). (D) Chaparro et al. (2007: fig. 9). (E) van Bocxlaer et al. (2010: fig. S1). (F) Pyron and Wiens (2011: fig. 2). (G) Pyron (2014: suppl. information "amph_shl.tre"). (H) Pereyra et al. (2016a: fig. 3 and appendix S12). (I) Jetz and Pyron (2018: suppl. information "amph_shl_new.tre"). 
al., 2010; Pyron and Wiens, 2011; Pyron, 2014; Portik and Papenfuss, 2015; Jetz and Pyron, 2018). The previous hypotheses disagree about the phylogenetic placement of Rhinella, recovering it: (1) as closely related to Incilius and Anaxyrus, and deeply nested within an "old world" bufonid clade (Pauly et al., 2004; Frost et al., 2006; Pramuk, 2006; Chaparro et al., 2007; Pereyra et al., 2016a); (2) as sister taxon of a clade containing all the "old world" bufonid genera (van Bocxlaer et al., 2010); or (3) in a clade together with Anaxyrus + Incilius that is, in turn, sister taxon of the "old world" bufonid clade (Pyron and Wiens, 2011; Pyron, 2014; Portik and Papenfuss, 2015; Ron et al., 2015; Jetz and Pyron, 2018). As all alternative hypotheses have low support values for most relevant nodes around Rhinella, we preferred to sample a broad diversity of bufonid genera representing most of the phylogenetic diversity of the family to rigorously test the relationships and monophyly of the genus. Consequently, we targeted 36 species of 22 bufonid genera as outgroup taxa for the combined molecular dataset and 21 of these species for the phenotypic dataset. Outgroup sequences were obtained exclusively from GenBank (see appendices 1,2). Thus, in order to increase the number of included genes for outgroup terminals (considering that the number of sampled genes for the ingroup in this work was higher than previous phylogenetic analyses of Bufonidae), we combined sequences from different specimens of the same species to construct several composite outgroup terminals (see justification by Campbell and Lapointe, 2009). These composite terminals (see appendices 1,2 ) were constructed only when their uncorrected p-distances (UPDs) in the $16 \mathrm{~S}$ rRNA gene were less than $0.5 \%$, which is less than the estimated mean divergence observed between sister species of most anurans (Vences et al., 2005a; Fouquet et al., 2007b; Funk et al., 2011). In taxonomy, the exclusive use of pairwise distances and fixed thresholds is questionable (e.g., Will and Rubinoff, 2004; Grant et al., 2006; Meier et al., 2008), but they serve as a useful heuristic for species identification and, in the present context, reduce the risk of constructing composited terminals that could compromise the phylogenetic analy- sis. Moreover, preliminary analyses including all the sequences of both conspecific specimens recovered them as monophyletic with high support (parsimony jackknife supports $>97 \%$, see below).

\section{The Ingroup: RHINELLA}

We included 278 terminals representing 83 described species of Rhinella for the combined (molecular + phenotypic) dataset. For practical purposes, the included taxa are presented below in the species groups to which they were assigned by Duellman and Schulte (1992), but considering subsequent modifications to this proposal (details of the assignation of each species to species groups by different authors are given in appendix 5 ).

For the purposes of our analysis, we recognize the following seven species groups within Rhinella: the $R$. acrolopha Group, the $R$. crucifer Group, the $R$. granulosa Group, the $R$. margaritifera Group, the $R$. marina Group, the $R$. spinulosa Group, and the demonstrably paraphyletic " $R$. veraguensis Group." Moravec et al. (2014) also proposed the Rhinella festae Group to include three species of the former Rhamphophryne and four species of the paraphyletic $R$. veraguensis Group (see Pramuk, 2006; Chaparro et al., 2007; van Bocxlaer et al., 2010; Pyron and Wiens, 2011), which they recovered as a clade in their molecular phylogenetic analysis. Although this resolves the nonmonophyly of the analyzed species of the $R$. veraguensis Group, the authors did not diagnose either their $R$. festae Group or their restricted $R$. veraguensis Group or address the placement of the remaining species of the former Rhamphophryne. Given that recognizing the $R$. festae Group left many species of the former Rhamphophryne and $R$. veraguensis Group s.l. unassigned to any group due to the lack of diagnoses, we exclude the $R$. festae Group below.

Grant and Bolívar-G. (2014) proposed the Rhinella acrolopha Group to include the species previously assigned to Rhamphophryne. Although molecular phylogenetic analyses have consistently supported the monophyly of this group (albeit on the basis of a small fraction of its spe- 
cies; van Bocxlaer et al., 2010; Pyron and Wiens, 2011; Pyron, 2014; Jetz and Pyron, 2018), its recognition renders the $R$. veraguensis Group paraphyletic (see Pramuk, 2006; Chaparro et al., 2007; van Bocxlaer et al., 2010; Pyron and Wiens, 2011; Pyron, 2014; Jetz and Pyron, 2018). Thus, as discussed by Grant and Bolívar-G. (2014), the composition and diagnosis of the $R$. festae Group, the $R$. acrolopha Group, and the $R$. veraguensis Group are problematic and will be addressed in the context of our results. For the time being, we employ the putatively monophyletic $R$. acrolopha Group and demonstrably paraphyletic " $R$. veraguensis Group" only to characterize and provide background on the ingroup.

\section{The RHinella ACROLOPHa Group}

This group consists of 10 small to mediumsized species of Rhinella characterized by a projecting snout, small and inconspicuous parotoid macroglands, heavily ossified skull with some degree of co-osification, well-defined cranial crests (at least in some species), tympanic membrane and annulus absent (except in R. truebae), $\mathrm{m}$. levator mandibulae externus undivided with trigeminal nerve passing medial (deep) to the muscle, m. adductor longus absent, and large and unpigmented eggs (Trueb, 1971; Lynch and Renjifo, 1990; Grant and Bolívar-G., 2014). These species are distributed from southern Panama to southern Ecuador, and many of them are critically endangered (Rueda-Almonacid et al., 2004).

We sampled the following species: Rhinella acrolopha, $R$. festae, $R$. lindae, $R$. macrorhina, $R$. nicefori, $R$. paraguas, $R$. ruizi, and $R$. tenrec. We also included an undescribed species from Colombia (Rhinella sp. C sensu Machado et al., 2016). Sequences of $R$. macrorhina and $R$. rostrata available from GenBank (A. G. Gluesenkamp, unpublished) were not included because our preliminary analyses (data not shown) revealed that the sequences of the fragments of $12 \mathrm{~S}$ and $16 \mathrm{~S}$ rRNA genes of each specimen appear to be chimeric and/or contaminated with $R$. festae, and we cannot determine with certainty which sequences correspond to each taxon (see also Cusi et al., 2017). Tissues samples of $R$. rostrata were not available for this study. This poorly known species (Noble, 1920) was described from "Santa Rita Creek," $23 \mathrm{~km} \mathrm{~N}$ of Mesopotamia town, in the southern part of the departamento de Antioquia, Colombia. There is great uncertainty about this locality, because it has never been possible to locate or document it in the literature a stream with that name near Mesopotomia (today part of the municipality of La Unión, Antioquia). Additionally, we could not obtain samples of $R$. truebae, a species known only from the holotype and for which the precise locality is unknown (Lynch and Renjifo, 1990; Vélez-Rodríguez, 2004a).

\section{The RHINELla CRUCIFER Group}

This putatively monophyletic species group is currently composed of six medium-sized species whose distribution is mainly associated with the Atlantic Forest of Argentina, Brazil, and Paraguay (Duellman and Schulte, 1992; Baldissera et al., 2004; Thomé et al., 2010, 2012; Roberto et al., 2014). The following characters have been proposed to diagnose this species group: skull heavily ossified with slightly elevated cranial crests, dorsal skin smooth with low, scattered tubercles, lateral row of enlarged tubercles present, pale mid vertebral line well-defined, and parotoid macroglands elongated, moderate in size (Duellman and Schulte, 1992; Baldissera et al., 2004; Pramuk, 2006). This species group was recognized as distinct from the Rhinella marina Group by R.F. Martin (1972b) and Duellman and Schulte (1992) and all its forms were considered as a single species (Bufo crucifer) for a long time (see Lutz, 1934; Cochran, 1955; Cei, 1980; Duellman and Schulte, 1992).

Baldissera et al. (2004) revised the taxonomy of this species group and recognized five species based on morphology and morphometrics: Rhinella abei (Baldissera et al., 2004), R. crucifer (Wied, 1821), $R$. henseli (Lutz, 1934), R. ornata (Spix, 1824), and $R$. pombali (Baldissera et al., 2004). Subsequent to the revision of Baldissera et al. (2004), two additional species, Rhinella inopina and $R$. casconi, were 
described from wet forests within the Cerrado and Caatinga habitats of Brazil, respectively (Vaz-Silva et al., 2012; Roberto et al., 2014). Pramuk (2006) only included one species ( $R$. ornata, as Bufo cruci$f e r)$ of this group in her phylogenetic analysis, and recovered it as the sister taxon of the $R$. marina Group. Thomé et al. $(2010,2012)$ corroborated the monophyly of the $R$. crucifer Group although the outgroup sampling was limited. They also highlighted problems in the taxonomy proposed by Baldissera et al. (2004), as the recognized species did not fully correspond with genetic structuring in the group. Thomé et al. $(2010,2012)$ found that samples from specimens identified as $R$. pombali are nested within $R$. crucifer and/or R. ornata in the mitochondrial phylogenies and are associated with intermediate nuclear genomes in nonphylogenetic analysis (see factorial correspondence analyses [FCA] in Thomé et al., 2012). In addition to these results, a geographic distribution between that of $R$. crucifer and $R$. ornata (Baldissera et al., 2004) is congruent with $R$. pombali as a hybrid complex between the last two species (Thomé et al., 2010, 2012). Furthermore, samples from $R$. abei were nested within $R$. ornata. Thomé et al. (2012) proposed to synonymize $R$. pombali with both parental species and suggested further reassessment of the taxonomic status of $R$. abei with additional molecular markers. Their results were congruent with $2 \mathrm{D}$ geometric morphometrics of the skull performed by Bandeira et al. (2016), who found R. pombali to be morphologically intermediate between $R$. crucifer and $R$. ornata, and $R$. abei nested within $R$. ornata in the multivariate space.

Several specimens of the six valid species (Rhinella abei, R. casconi, R. crucifer, R. henseli, $R$. inopina, and R. ornata) were included in our analyses to test the monophyly of this group and the results of Thomé et al. $(2010,2012)$. We carried out a preliminary analysis (data not shown) including additional nuclear and mitochondrial sequences of two specimens of " $R$. pombali" and the results supported their findings (see Hybridization and genetic introgression in Rhinella section), so we did not include specimens of " $R$. pombali" in our subsequent analyses.

\section{The Rhinella granulosa Group}

This monophyletic species group is currently composed of 14 medium- to small-sized species of Rhinella (Pramuk, 2006; Pereyra et al., 2016a; Murphy et al., 2017). The following characters have been proposed to diagnose this species group: skull heavily ossified and exostosed with low, granular or elevated cranial crests, dorsal skin with small, keratinous-tipped tubercles, and lateral row of enlarged tubercles absent (Gallardo, 1957, 1965; R.F. Martin, 1972a, 1972b; Cei, 1980; Duellman and Schulte, 1992; Pramuk, 2006). All species of the R. granulosa Group are mostly distributed in open areas of South America and Panama (Gallardo, 1965; Duellman and Schulte, 1992; Duellman, 1999; Narvaes and Rodrigues, 2009; Sanabria et al., 2010).

The taxonomy of this species group was first revised by Gallardo (1965) and more recently by Narvaes and Rodrigues (2009). The latter authors recognized and diagnosed 12 species on the basis of morphological and morphometrical analyses. Subsequently, Sanabria et al. (2010) described a new species (R. bernardoi) from San Juan, western Argentina. The phylogenetic analyses of Pramuk (2006) and Pereyra et al. (2016a), comprising very different samples of species and characters, recovered this species group as monophyletic and discussed several of its phenotypic synapomorphies. Moreover, Pereyra et al. (2016a) documented the occurrence of hybridization between sympatric species as well as past mitochondrial introgression and proposed several morphological synapomorphies for the group. Vera Candioti et al. (2016) proposed some additional synapomorphies from the embryonic morphology (a very short third pair of gills, type A adhesive glands, the adhesive gland subdivision immediately before the gills reach their maximum development, and a short dorsal line of hatching glands mostly restricted to the cephalic region). More recently, Murphy et al. (2017) found the populations of $R$. humboldti on both sides of the Andes to be phylogenetically distinct, leading 
them to restrict $R$. humboldti to the western Andean populations and resurrect $R$. bebeei for the eastern ones.

In our phylogenetic analyses, we included most species of this group (Rhinella azarai, $R$. beebei, $R$. bergi, $R$. bernardoi, $R$. centralis, $R$. dorbignyi, $R$. fernandezae, $R$. granulosa, $R$. humboldti, $R$. major, $R$. merianae, $R$. mirandaribeiroi, and $R$. pygmaea) with the exception of $R$. nattereri, a species known from a restricted area in the border between Brazil, Guyana, and Venezuela (Bokermann, 1967; Narvaes and Rodrigues, 2009).

\section{The Rhinella margaritifera Group}

The definition of this species group is controversial, as diagnoses have been largely based on morphological variation of the Rhinella margaritifera species complex (e.g., R.F. Martin, 1972b; Hoogmoed, 1986; Pramuk, 2006) or subjective notions of similarity without consideration of character polarity (e.g., Cei, 1972a; Hoogmoed, 1990; Duellman and Schulte, 1992). The following characters have been used to diagnose this species group: skull relatively lightly ossified with variable amounts of dermal ornamentation and prominent cranial crests, dorsal skin smooth or with small, scattered tubercles, and a lateral row of enlarged tubercles present (Hoogmoed, 1990; Duellman and Schulte, 1992; Vélez-Rodríguez, 2004b; Pramuk, 2006). Nevertheless, this definition does not accomodate the morphology of species recently included in the group (R. ocellata and $R$. yunga, the putative sister species to the remaining species of the group, see Moravec et al., 2014).

Similarly, the taxonomy of the species of the Rhinella margaritifera Group is also conflicted due to imprecise type localities, extreme sexual dimorphism, and the extensive ontogenetic variation that hinder the specific recognition and description of some putative undescribed species (Hoogmoed, 1977; 1986; 1990; Hass et al., 1995; De la Riva et al., 2000; Vélez-Rodríguez, 2004b; Fouquet et al., 2007a, 2007b, 2007c; Lavilla et al., 2013, 2017). Currently, this group is composed of 20 medium-sized species (see appendix 5) dis- tributed from Panama to southern Brazil, including the Amazonia and Guiana Shield.

We sampled 17 species of this group: Rhinella acutirostris, R. alata, R. castaneotica, R. dapsilis, $R$. gildae, $R$. hoogmoedi, $R$. lescure $i, R$. magnussoni, $R$. margaritifera, $R$. martyi, $R$. ocellata, $R$. paraguayensis, $R$. proboscidea, $R$. scitula, $R$. sclerocephala, R. stanlaii, and $R$. yunga. This sampling also includes numerous specimens of the $R$. margaritifera species complex throughout its distribution. Additionally, we included two undescribed species of this group, one from Ecuador and another one from Colombia, Peru, and Venezuela. Two species of this group were unsampled: $R$. roqueana, which occurs along the lowlands east of the Andes in southern Ecuador and adjacent northern Peru (Hoogmoed, 1990), and $R$. sebbeni, which is known only from a few localities of the riparian and dry seasonal forests in the Cerrado biome (Vaz-Silva et al., 2015).

\section{The RHINeLla MARINA Group}

This species group is currently composed of 11 large species (Duellman and Schulte, 1992; Maciel et al., 2010; Vallinoto et al., 2010; Lavilla and Brusquetti, 2018). The group is distributed from the southern United States to Argentina, and its species inhabit both open and forested areas (Duellman and Schulte, 1992; Frost, 2020). The following characters have been proposed as diagnostic of this species group: extremely ossified and exostosed skulls, elevated (keratinized or not) cranial crests, dorsal skin with small and large tubercles, and lateral row of enlarged tubercles absent (Duellman and Schulte, 1992; Pramuk, 2006; Maciel et al., 2010). Maciel et al. (2010) and Vallinoto et al. (2010) studied the phylogenetic relationships in this species group. Maciel et al. (2010) included phenotypic (morphological and parotoid-macrogland secretions) and molecular (sequences of three mitochondrial and one nuclear genes) characters and found this group as monophyletic, being the sister taxon of the Rhinella crucifer Group. Alternatively, Vallinoto et al. (2010) found the R. crucifer Group nested within the $R$. marina Group. Sequeira et al. (2011) reported 
the occurrence of extensive unidirectional introgression between $R$. diptycha (as R. schneideri) and some populations of $R$. marina that could contribute to biased inferences in the phylogenetic relationships. More recently, Vallinoto et al. (2017) reevaluated this hypothesis by including additional samples and molecular markers and found a more complex scenario with no evident pattern of unidirectional introgression and a doubtful taxonomic status of some $R$. marina populations. Finally, based on a phylogenetic analysis using mitochondrial genes and morphometric data, Acevedo et al. (2016) resurrected $R$. horribilis for the western Andean populations previously considered $R$. marina. Recently Bessa-Silva et al. (2020) found evidence of interspecific nuclear differentiation between these species and a marked discordance between mitochondrial and nuclear phylogenetic inferences in the R. marina Group.

We included samples of several populations from all the currently recognized species of this group: Rhinella achavali, $R$. arenarum, $R$. cerradensis, $R$. diptycha, $R$. horribilis, $R$. icterica, $R$. jimi, $R$. marina, $R$. poeppigii, $R$. rubescens, and $R$. veredas. For $R$. arenarum, we also included samples of the populations historically assigned to the subspecies $R$. arenarum mendocina (see Laurent, 1969).

\section{The Rhinella spinulosa Group}

Nine species are currently assigned to this group, which are distributed in the Andean region from southern Ecuador to southern Argentina and Chile, except for Rhinella achalensis, which is endemic to the Sierras Pampeanas Centrales in central Argentina (Cei, 1972b; Pramuk and Kadivar, 2003). The species of this group are medium sized and have a moderately to lightly ossified skull that lacks dermal sculpturing and exostosis. They also have a marked sexual dimorphism in skin texture and coloration (Vellard, 1959; Cei, 1972a, 1972b; Duellman and Schulte, 1992). This group was recovered as monophyletic in the combined phylogenetic analysis of Pramuk (2006: fig. 4) but paraphyletic in the separate molecular or morphological anal- yses (Pramuk, 2006: figs. 1-3). Some subspecies have been recognized for the nominal species of this group, which is a putative species complex (Vellard, 1959; Cei, 1972a; Ferraro et al., 2018).

We included all recognized species of this group: Rhinella achalensis, $R$. amabilis, $R$. arequipensis, $R$. arunco, $R$. atacamensis, $R$. limensis, $R$. rubropunctata, $R$. spinulosa (including populations historically assigned to the subspecies $R$. $s$. papillosa, R. s. spinulosa, and R. s. trifolium), and $R$. vellardi. We were unable to sample populations assigned to two subspecies of $R$. spinulosa: $R$. s. altiperuviana and R. s. flavolineata.

\section{The "Rhinella veraguensis Group"}

This nonmonophyletic group is composed of 17 small- to medium-sized species, all of which occur in the cloud forest of the Andes from northern Peru to northern Argentina, excepting Rhinella chrysophora, a species from north-central Honduras (Cei, 1972a; Duellman and Schulte, 1992; Chaparro et al., 2007; Cusi et al., 2017; McCranie, 2017). Members of this group are morphologically diverse with terrestrial, semiaquatic, or arboreal habits.

The following characters have been considered diagnostic for the Rhinella veraguensis Group: skull with weak exostosis, cranial crests absent or weak, dorsal skin bearing small elevated tubercles, and a lateral row of enlarged tubercles in some species (Gallardo, 1961; Cei, 1972a; Duellman and Schulte, 1992; Pramuk, 2006). This group has been consistently recovered as nonmonophyletic (Pramuk, 2006; Chaparro et al., 2007; van Bocxlaer et al., 2010; Pyron and Wiens, 2011; Moravec et al., 2014; Pyron, 2014; Cusi et al., 2017; Jetz and Pyron, 2018) and its definition and composition are problematic (see Pereyra et al., 2015; and comments regarding the definition of the $R$. acrolopha and $R$. festae Groups above). For purposes of description of the ingroup, $R$. lilyrodriguezae is included in this group (according to the phylogenetic relationships recovered by Cusi et al., 2017), although this species was assigned to the $R$. festae Group in the original 
description. We included samples of most species of this group ( $R$. amboroensis, $R$. arborescandens, $R$. chavin, $R$. fissipes, $R$. inca, $R$. justinianoi, $R$. leptoscelis, $R$. lilyrodriguezae, $R$. manu, $R$. multiverrucosa, $R$. nesiotes, $R$. quechua, $R$. rumbolli, $R$. tacana, $R$. veraguensis, and $R$. yanachaga). The only unsampled species was $R$. chrysophora, which occurs in the Wet Forest in the central and western portions of the Cordillera Nombre de Dios, central-north Honduras. This species has not been observed since 1996 and is thought to be extinct (McCranie, 2017).

\section{Species Unassigned to Any Group}

Six species of Rhinella are not currently assigned to any group (for a history of previous group assignments see appendix 5). Among them, we included $R$. ceratophrys, R. gallardoi, and R. sternosignata in our phylogenetic analyses. The following species were not included in the phylogenetic analyses: (1) R. cristinae (Vélez-Rodríguez and RuizCarranza, 2002), a species known only from the type locality (Vereda Tarquí, km 53-54 on road Altamira-Florencia, Municipio de Florencia, Departamento del Caquetá, Colombia) and collected in 1990 for the last time; (2) R. gnustae (Gallardo, 1967), which is only known from the holotype collected in 1925 from an imprecise locality (Rio Grande, Jujuy, Argentina; see Lavilla et al., 2002); and (3) R. iserni (Jiménez de la Espada, 1875), which is also known with certainty only from the holotype and its type locality is imprecise (Andes de Chanchamayo, Peru).

\section{Molecular Data}

\section{Tissue SAmpling}

The molecular data were the main source of evidence in terms of both number of scored characters and sampled terminals. As one of the main goals of this paper was to test the monophyly of all the species groups of Rhinella, we attempted to obtain tissue samples from as many species as possible, with particular emphasis on putative nonmonophyletic species groups. Additionally, we included specimens from multiple populations of species that include recognized subspecies (e.g., $R$. arenarum and $R$. spinulosa), species that might represent species complexes (e.g., $R$. dapsilis, $R$. margaritifera, and $R$. proboscidea), and widely distributed taxa (e.g., $R$. diptycha, $R$. marina, and $R$. veraguensis) to evaluate their taxonomy. We included GenBank sequences only in cases where precise voucher number and locality data are provided, for specimens sequenced for at least the $16 \mathrm{~S}$ rRNA gene. Besides, we made an effort to corroborate the identity of most relevant vouchers. A detailed list of all the terminals included in our analyses is given in appendices 1 and 2 .

\section{LABORATORY Protocols}

We extracted total genomic DNA from ethanol-preserved tissues (liver, muscle, or fingertips) using the Qiagen DNeasy kit. We carried out PCR amplifications in a total volume of $25 \mu \mathrm{l}$ reactions using $0.2 \mu \mathrm{l} \mathrm{Taq} \mathrm{(Fermentas).} \mathrm{The} \mathrm{PCR} \mathrm{protocol}$ consisted of an initial denaturation step of $3 \mathrm{~min}$. at $94^{\circ} \mathrm{C}$ followed by 35 (for mitochondrial genes) or 45 (for nuclear genes) cycles consisting of 30 seconds at $94^{\circ} \mathrm{C}$ for denaturation, 40 seconds at $48^{\circ}-62^{\circ} \mathrm{C}$ for annealing, and $30-60$ seconds at $72^{\circ}$ $\mathrm{C}$ for extension, and a final extension step of 10-15 minutes at $72^{\circ} \mathrm{C}$. We cleaned PCR-amplified products using $10 \mathrm{U}$ of Exonuclease plus $1 \mathrm{U}$ of alkaline phosphatase per reaction. We sequenced the products with an automatic sequencer ABI 3730XL (Applied Biosystems) in both directions to check for potential errors and nuclear polymorphisms. We processed the chromatograms using the software Sequencher version 4.5 (Gene Codes, Ann Arbor, MI) and edited the complete sequences with BioEdit (Hall, 1999). Sequences are deposited in GenBank under the accession numbers MW002838-MW003700.

\section{GENOTYPIC CHARACTER SAMPLING}

The mitochondrially encoded loci sampled for the phylogenetic analyses include: (1) the $12 \mathrm{~S}$ 
rRNA, the tRNA Valine, and the 16S rRNA genes (12S-tRNA ${ }^{\text {Val }}$-16S; 2469 bp), (2) a fragment comprising the upstream section of the $16 \mathrm{~S}$ rRNA gene and the tRNA Leucine, NADH dehydrogenase subunit 1 , and tRNA Isoleucine genes (16S- $t R N A^{\text {Leu }}$ ND1- tRNA $A^{\text {Ile; }} 1305 \mathrm{bp}$ ), and (3) a fragment of cytochrome $b$ gene (cytb; $700 \mathrm{bp}$ ), for a total of up to $4474 \mathrm{bp}$. The nuclear loci include: (1) the C-X-C motif chemokine receptor 4 gene ( $c x c r 4 ; 676 \mathrm{bp})$, (2) the solute carrier family 8 member A1 gene (slc8a1; $715 \mathrm{bp}$ ), (3) the proopiomelanocortin gene (pomc; $559 \mathrm{bp}$ ), (4) two nonoverlapping fragments of the recombination activating 1 gene ( $r a g 1-\mathrm{a}$ and rag1-b; 936 and 429 bp respectively), and (5) the rhodopsin gene ( $r h o ; 316 \mathrm{bp}$ ), for a total of $3631 \mathrm{bp}$. Primers and their sources are detailed in table 1.

For the parsimony total evidence and maximum-likelihood (ML) analyses (see below), the amount of sequence data analyzed per terminal ranged from 447 bp (Rhinella gildae URCA 12651 obtained from GenBank) to 8089 bp ( $R$. henseli CFBH 20117), with a mean of 4378 bp per terminal. All the phylogenetic datasets employed in the analyses are available at https:// doi.org/10.5531/sd.sp.46).

\section{PHENOTYPIC DATA}

The phenotypic dataset consisted of direct observations on specimens and bibliographic information for 90 characters, scored for 106 terminals ( 84 from the ingroup, 22 from outgroups). The scoring was recorded using Mesquite version 3.51 (Maddison and Maddison, 2018). The dataset was assembled from the following character systems: 33 from adult osteology, 15 from hand and foot musculature, 3 from the tympanic middle ear, 1 from adult visceral anatomy, 15 from adult external morphology, 9 from larval external morphology, 3 from larval chondrocranium, 4 from embryonic external morphology, 6 from natural history, and 1 from cytogenetics. Phenotypic characters are described below (see List and Description of Characters); the phenotypic matrix is included as supplementary data 1 (available at https:// doi.org/10.5531/sd.sp.46).

Cranial and postcranial osteology follows the terminology employed by Trueb (1973, 1993), that of cranial crests follows Mendelson (1997a), and hand and foot myology follows Blotto et al. (2020). Terminology for larval external morphology follows Altig and McDiarmid (1999) and the characterization of embryonic structures follows Nokhbatolfoghahai and Downie (2005, 2008). Osteology was studied in (1) cleared and doublestained specimens prepared following the techniques of Wassersug (1976), (2) dry skeletons, and (3) $\mu$-CT scans (available for download at www. morphosource.org, Duke University). Additional information was obtained from detailed osteological descriptions in the literature (see appendix 3). Visualization and data processing of $\mu$-CT images was done in MeshLab (Cignoni et al., 2008). For the study of myology, dissections of the hand and foot musculature were performed to remove superficial layers and observe successively deeper muscles as outlined by Blotto et al. (2020). Topical applications of the iodine/potassium iodide solution of Bock and Shear (1972) were used when necessary to enhance contrast. The remaining characters were scored from the literature, unless specified (see appendix 3 ).

We scored multiple states for uncertainty or ambiguity in the condition of a terminal (among some states, but not all the character states) for some characters (see Pol and Apesteguía, 2005). This way of scoring let us incorporate relevant information (mainly from descriptions obtained from the bibliography) even when descriptions were not detailed enough. For 19 series of transformation, we used composite coding (sensu Maddison, 1993), which minimizes the occurrence of inapplicable or missing entries (Pimentel and Riggins, 1987; Maddison, 1993; Strong and Lipscomb, 1999).

\section{Phylogenetic Analyses}

The final taxon sample for the phylogenetic analyses was defined by means of a series of 
preliminary analyses that clarified the situation of many problematic terminals. As hybridization and genetic introgression, both nuclear and mitochondrial, seem to be common in some species of Rhinella (Sequeira et al., 2011; Pereyra et al., 2016a; Vallinoto et al., 2017), we first performed exploratory analyses of mitochondrial (MD) and nuclear (ND) datasets independently to detect nuclear-mitochondrial discordance as indicative of putative genetic introgression. Subsequently, we performed a total evidence (TE) analysis (Kluge, 1989, 2004; Nixon and Carpenter, 1996) combining nonintrogressed nuclear and mitochondrial sequences and the phenotypic dataset (see details in appendix 2).

The phylogenetic analyses of each separate molecular dataset (nuclear and mitochondrial, see below) and the total evidence analysis were performed in TNT version 1.5 (Goloboff et al., 2008; Goloboff and Catalano, 2016). Gaps were considered as a fifth state in all parsimony analyses (nuclear, mitochondrial, and total evidence analyses) and all classes of transformation events were equally weighted. In addition, we performed a total evidence analysis considering gaps as missing data for comparisons with the maximum likelihood analysis (see below). Unless otherwise stated, all results shown refer to parsimony analyses in which gaps were treated as a fifth state. We favoured parsimony as optimality criterion because the cladogram that minimizes transformations to explain the observed variation is the simplest one, maximizes evidential congruence, and has the greatest explanatory power (Farris, 1983; Goloboff, 2003; Goloboff and Pol, 2005; Kluge and Grant, 2006; Wheeler et al., 2006). Sequences were aligned using the online software MAFFT v7 (Katoh and Toh, 2008; Katoh et al., 2019) under the strategy E-INS-i (for the 12S-tRNA $A^{V a l}-16 S$ fragment) and L-INS-i or G-INS-i (for remaining fragments), with default parameters for gap opening and extension. These alignments were used for both phylogenetic analyses and clade supports estimations (see details below).
Separate Phylogenetic Analyses of Nuclear and Mitochondrial Sequences

Both nuclear and mitochondrial datasets were analyzed in TNT using "New Technology" searches and performing a combination of sectorial searches, ratchet, and tree fusing (Goloboff, 1999; Nixon, 1999), using the default settings for these strategies. Tree searches were performed until the consensus was stabilized 10 times, with a factor of 75 (see Goloboff, 1999; Giribet, 2005).

The strict consensus tree resulting from the analysis of all sampled taxa of the nuclear dataset (= ND) was poorly resolved (data not shown). A poor resolution of the consensus can be due to the effect of just a small number of wildcard or rogue taxa, which are those that assume varying phylogenetic positions in the most parsimonious trees (MPT) (Nixon and Wheeler, 1992; Wilkinson, 1996; Aberer et al., 2013; Goloboff and Szumik, 2015). To avoid including terminals that act as wildcard taxa due to the lack of evidence, we included only terminals with more than three nuclear sequenced fragments (see appendix 2). Although there is an imperfect relationship between missing data and wildcard behavior, we identified three loci as the critical number to obtain an informative and comparable consensus in preliminary analyses. After excluding terminals with fewer than three nuclear fragments from the dataset, we reanalyzed this restricted nuclear dataset (rND) to estimate the consensus tree and clade supports (see below). The mitochondrial dataset was analyzed using the same terminals as the restricted nuclear dataset (i.e., restricted mitochondrial dataset, $\mathrm{rMD}$ ) and similar parameters of analysis (see above), to allow the comparison.

\section{Total Evidence Analysis}

For the TE analysis, we followed the strategy described above for the separate nuclear and mitochondrial analyses. In this analysis, we included: (1) all the nuclear sequences from the complete nuclear dataset, (2) all the mitochondrial sequences 
TABLE 1

Primers used to amplify and sequence DNA in this study See appendix 2 for gene abbreviations.

\begin{tabular}{|c|c|c|c|c|c|}
\hline Genome & Gene & Primer & Direction & Primer sequence $5^{\prime} \rightarrow 3^{\prime}$ & Source \\
\hline \multirow{18}{*}{ Mitochondrial } & \multirow{12}{*}{$\begin{array}{l}12 S \\
16 S\end{array}$} & MVZ59 & Forward & ATAGCACTGAAAAYGCTDAGATG & Graybeal, 1997 \\
\hline & & Phe2-L & Forward & AAAGCATAACACTGAAGATGT'TAAGATG & Wiley et al., 1998 \\
\hline & & $12 \mathrm{~S} F-\mathrm{H}$ & Reverse & CTTGGCTCGTAGTTCCCTGGCG & Goebel et al., 1999 \\
\hline & & $12 \mathrm{~S} \mathrm{~A}-\mathrm{L}$ & Forward & AAACTGGGATTAGATACCCCACTAT & Goebel et al., 1999 \\
\hline & & tRNAval-H & Reverse & GGTGTAAGCGARAGGCTTTKGTTAAG & Goebel et al., 1999 \\
\hline & & $12 \mathrm{Sm}$ & Forward & GGCAAGTCGTAACATGGTAAG & Pauly et al., 2004 \\
\hline & & $\mathrm{L} 13$ & Forward & TTAGAAGAGGCAAGTCGTAACATGGTA & Feller and Hedges, 1998 \\
\hline & & Titus I & Reverse & GGTGGCTGCTTTTAGGCC & Titus and Larson, 1996 \\
\hline & & $\mathrm{L} 2 \mathrm{~A}$ & Forward & CCAAACGAGCCTAGTGATAGCTGGTT & Hedges, 1994 \\
\hline & & $\mathrm{H} 10$ & Reverse & TGATTACGCTACCTTTGCACGGT & Hedges, 1994 \\
\hline & & $\mathrm{AR}$ & Forward & CGCCTGTTTATCAAAAACAT & Palumbi et al., 1991 \\
\hline & & Wilkinson2 & Reverse & GACCTGGATTACTCCGGTCTGA & Wilkinson et al., 1996 \\
\hline & \multirow{4}{*}{$n d 1$} & $16 \mathrm{~S}$-frog & Forward & TTACCCTRGGGATAACAGCGCAA & Wiens et al., 2005 \\
\hline & & tMet-frog & Reverse & TTGGGGTATGGGCCCAAAAGCT & Wiens et al., 2005 \\
\hline & & ND1 F1 & Forward & AGCCATAATCATCTGAACC & Smith et al., 2005 \\
\hline & & ND1 R1 & Reverse & TCCTCCCTATCAAGGAGGTCC & Smith et al., 2005 \\
\hline & \multirow{2}{*}{$c y t b$} & CytbDen3-L & Forward & AAYATYTCCRYATGATGRAAYTTYGG & $\begin{array}{l}\text { Santos and Cannatella, } \\
2011\end{array}$ \\
\hline & & $\begin{array}{l}\text { CytbDen 1- } \\
\mathrm{H}\end{array}$ & Reverse & GCRAANAGRAAGTATCATTCNGGYTTRAT & $\begin{array}{l}\text { Santos and Cannatella, } \\
2011\end{array}$ \\
\hline \multirow{15}{*}{ Nuclear } & \multirow{2}{*}{$\operatorname{cxcr} 4$} & CXCR4-C & Forward & GTCATGGGCTAYCARAAGAA & Biju and Bossuyt, 2003 \\
\hline & & CXCR4-G & Reverse & AGGCAACAGTGGAARAANGC & Biju and Bossuyt, 2003 \\
\hline & \multirow{3}{*}{ pomc } & POMC-1 & Forward & GAATGTATYAAAGMMTGCAAGATGGWCCT & Wiens et al., 2005 \\
\hline & & POMC-2 & Reverse & TAYTGRCCCTTYTTGTGGGCRTT & Wiens et al., 2005 \\
\hline & & POMC-2B & Reverse & GCATTYTTGAAAAGAGTCATTARTGGAGTCTG & Pramuk, 2006 \\
\hline & \multirow{2}{*}{ ragla } & MartFl1 & Forward & AGCTGCAGYCARTAYCAYAARATGTA & Hoegg et al., 2004 \\
\hline & & AmpR1 & Reverse & AACTCAGCTGCATTKCCAATRTCA & Hoegg et al., 2004 \\
\hline & \multirow{4}{*}{ raglb } & R1-GFF & Forward & GAGAAGTCTACAAAAAVGGCAAAG & Faivovich et al., 2005 \\
\hline & & R1-GFR & Reverse & GAAGCGCCTGAACAGTTTATTAC & Faivovich et al., 2005 \\
\hline & & RAG1 TG1F & Forward & CCAGCTGGAAATAGGAGAAGTCTA & Grant et al., 2006 \\
\hline & & RAG1 TG1R & Reverse & CTGAACAGTTTATTACCGGACTCG & Grant et al., 2006 \\
\hline & \multirow{2}{*}{ rho } & Rhod1A & Forward & ACCATGAACGGAACAGAAGGYCC & $\begin{array}{l}\text { Bossuyt and Milinkov- } \\
\text { itch, } 2000\end{array}$ \\
\hline & & Rhod1C & Reverse & CCAAGGGTAGCGAAGAARCCTTC & $\begin{array}{l}\text { Bossuyt and Milinkov- } \\
\text { itch, } 2000\end{array}$ \\
\hline & \multirow{2}{*}{ slc $8 a 1$} & NACAL & Forward & TCCAAAGCAGATATTGAAATGGA & $\begin{array}{l}\text { Roelants and Bossuyt, } \\
2005\end{array}$ \\
\hline & & NACAO & Reverse & ATACCTGCATGATCATCATCAAA & $\begin{array}{l}\text { Roelants and Bossuyt, } \\
2005\end{array}$ \\
\hline
\end{tabular}


from the complete mitochondrial dataset, and (3) the phenotypic dataset. The following criteria were used to treat putatively conspecific sequences as pertaining to the same or different terminals: (1) sequences from the same individual or conspecific individuals placed in well-supported discordant positions in the separate nuclear and mitochondrial analyses were considered as independent terminals, because discordance suggests mitochondrial introgression between different species (see Pereyra et al., 2016a); and (2) terminals from the phenotypic dataset were combined with the more closely related conspecific terminal of the molecular dataset (mitochondrial + nuclear). When mitochondrial and nuclear sequences of a specimen were included separately, the phenotypic data were combined with the nuclear sequences. Appendix 2 provides a list of all the terminals included and excluded in the TE analysis.

\section{Resampling Support Measures}

Two types of resampling support measures were estimated for the datasets in TNT version 1.5 (Goloboff and Catalano, 2016): (1) parsimony jackknife absolute frequencies (JAF; Farris et al., 1996) and (2) parsimony jackknife frequency differences (JGC; Goloboff et al., 2003). For estimation of both measures, we performed 1,000 replicates using "New Technology" searches consisting of a combination of sectorial searches, ratchet, and tree fusing (Goloboff, 1999; Nixon, 1999), reaching minimum length two times (preliminary analyses showed that minimum lengths are hit with this search strategy). Goloboff et al. (2003) noted that the resampling support for a clade does not necessarily correlate with the absolute frequency itself (i.e., the number of times a group is recovered in the resampled matrices), because groups with positive support $(\geq 50 \%)$ can have much lower frequencies than groups with no support at all $(<50 \%)$. To solve this situation, these authors proposed to also consider the value GC (i.e., frequency difference), which indicates the frequency differences between a group and the most frequent contradictory group. Values of this score range between -100\% (maximum contradiction) and 100\% (maximum support).

\section{Maximum-Likelihood Analysis}

Maximum-likelihood analysis was performed with IQ-TREE v1.6.12 (Nguyen et al., 2015) considering the same dataset (DNA sequences + phenotypic characters) as the TE analysis under parsimony. ModelFinder (Kalyaanamoorthy et al., 2017), which is implemented in IQ-TREE, was used to select the optimal partition scheme and substitution models for molecular characters. ModelFinder implements a greedy strategy (Lanfear et al., 2012) that starts with the full partition model and subsequentially merges two genes until the model fit does not increase any further. The best partition scheme included two subsets (see table 2). For morphological data we use the two morphological ML models (see Lewis, 2001) implemented in IQ-TREE (i.e., MK and ORDERED, for unordered and ordered characters respectively) considering the ascertainment bias correction (ASC) method. We consider edgelinked-proportional partition model but separate substitution models and rate evolution between partitions (-spp option). The maximum-likelihood tree was conducted with 1000 ultrafast bootstrap replicates (Minh et al., 2013; Hoang et al., 2018) using the option -bnni that reduces the risk of overestimating branch supports due to severe model violations. The resulting tree was visualized and edited in FigTree 1.4.3 (Rambaut, 2016). Partitions and models selected are detailed in table 2.

\section{TAXonomic Evaluation}

We considered the following criteria in assessing the taxonomic status of each lineage: (1) the cladogram topology resulting from the phylogenetic analyses, (2) the uncorrected pairwise distances (UPDs) of a fragment of the 16S rRNA gene (delimited by the primers AR and WILK2; see Vences et al., 2005a, 2005b; Fouquet et al., 2007b) calculated in PAUP* (Swofford, 2002), and (3) the known phenotypic evidence for each 


\section{TABLE 2}

Best partition scheme and best-fit models selected by ModelFinder for the molecular data. For phenotypic data, we used morphological models considering the ascertainment bias correction (ASC) method.

\begin{tabular}{lll}
\hline \hline Subset & Data blocks & Model \\
\hline 1 & $\begin{array}{l}\text { Coding mitochondrial sequences 1st, 2nd; Coding nuclear sequences } \\
\text { 1st, 2nd, 3rd; Non coding mitochondrial sequences }\end{array}$ & GTR+F+I+G4 \\
\hline 2 & Coding mitochondrial sequences 3rd & TN+F+I+G4 \\
\hline 3 & Unordered phenotypic characters & MK+ASC \\
\hline 4 & Ordered phenotypic characters & ORDERED+ASC \\
\hline
\end{tabular}

taxon. The phenotypic criterion was mainly considered in cases where relationships were unresolved (i.e. occurrence of polytomies) or poorly supported (JGC <50\%) within a clade. For estimation of UPDs, datasets containing only sequences of the 16S rRNA gene for each species group (as are redefined in the Results section) were aligned in MAFFT under the strategy G-INS-i.

\section{LIST AND DESCRIPTION OF CHARACTERS}

Characters modified from previous phylogenetic studies are indicated with an asterisk $\left(^{*}\right)$.

\section{Adult Osteology}

Most of the osteological characters used here are those of Pramuk (2006), so they are not described in detail except when relevant (e.g., when character states were modified or additional character states were considered). Described characters refer to adult individuals of both sexes unless specified.

\section{Skull}

0. Preorbital crest (on the maxillary process of nasal), occurrence: (0) absent or indistinguishable, (1) weak, (2) well developed. Additive. Cranial crests were considered osteological characters, although it could also be scored from whole-preserved specimens. The use of presence/ absence of cranial crests has a long history in bufonid taxonomy, and they were used in a phy- logenetic context by Pramuk (2006: chars. 63-69). However, unlike Pramuk (2006), we differentiate between weak and well-developed crests. State 1 (weak) refers to cranial crests that are faint or not evident externally in living or intact preserved specimens, but evident in osteological preparations. State 2 (well developed) refers to crests that are evident externally in both intact and osteologically prepared specimens. When osteological preparations were not available to precisely determine the absent or weak state of the crest (since both states are similar in complete specimens) we scored these uncertainties as multiple states (i.e., 0/1, see Phenotypic data scoring in Material and methods section).

Previous USAGe In Phylogenetic Studies: Inger (1972: char. 29*), Morrison (1994: char. $13^{*}$ ), Mendelson (1997a: char. $\left.6^{*}\right)$, Pramuk (2006: char. $\left.65^{\star}\right)$, Mendelson et al. (2011: char. $6^{*}$ ).

1. Supraorbital crest (on frontoparietals), occurrence: (0) absent or indistinguishable, (1) weak, (2) well developed. Additive.

Previous usage In Phylogenetic StUdies: Inger (1972: char. 29*), Morrison (1994: char. $14^{*}$ ), Mendelson (1997a: char. $\left.7^{*}\right)$, Pramuk (2006: char. $\left.68^{\star}\right)$, Mendelson et al. (2011: char. $7^{\star}$ ).

2. Pretympanic crest (on the zygomatic ramus of squamosal), occurrence: (0) absent or indistinguishable, (1) weak, (2) well developed. Additive.

Previous USAGE IN PHylogenetic STUdies: Morrison (1994: char. 16*), Mendelson (1997a: char. $\left.11^{\star}\right)$, Pramuk (2006: char. $\left.66^{*}\right)$, Mendelson et al. (2011: char. $\left.11^{\star}\right)$. 
3. Supratympanic crest (on the otic ramus of squamosal), occurrence in females: (0) supratympanic crest inconspicuous or developed, but that does not extend beyond the level of the cranial roof dorsally, (1) supratympanic crest hypertrophied extending beyond the level of the cranial roof dorsally. This character was codified separately for males and females since a dimorphic condition was detected. Large supratympanic crest occurs mainly in adult females of many species of the Rhinella margaritifera Group (Hoogmoed, 1990; Duellman and Schulte, 1992). However, males of some of these species also have large supratympanic crest (Hoogmoed, 1990).

Previous USAGe IN PhylogenetiC STUdies: Morrison (1994: char. 17*), Mendelson (1997a: char. $10^{*}$ ), Vélez-Rodríguez (2004b: char. $35^{*}$ ), Pramuk (2006: char. 69*), Mendelson et al. (2011: char. $\left.10^{*}\right)$.

4. Supratympanic crest (on the otic ramus of squamosal), occurrence in males: (0) supratympanic crest inconspicuous or developed, but that does not extend beyond the level of the cranial roof dorsally, (1) supratympanic crest hypertrophied extending beyond the level of the cranial roof dorsally.

Previous usage in Phylogenetic StUdies: Morrison (1994: char. 17*), Mendelson (1997a: char. $10^{*}$ ), Vélez-Rodríguez (2004b: char. $35^{*}$ ), Pramuk (2006: char. 69*), Mendelson et al. (2011: char. $\left.10^{*}\right)$.

5. Parietal crest (on frontoparietal), occurrence: (0) absent or indistinguishable, (1) weak, (2) well developed. Additive.

Previous USAGe in PHYLOGenetic STUdies: Inger (1972: char. 29*), Morrison (1994: char. $15^{\star}$ ), Mendelson (1997a: char. $\left.8^{\star}\right)$, Pramuk (2006: char. $\left.64^{*}\right)$, Mendelson et al. (2011: char. $8^{\star}$ ).

6. Nasals, shape of anterior margins: $(0)$ relatively blunt, (1) acuminate.

Previous USAGe IN PHYLOGENETIC STUdies: Mendelson (1997a: char. 34*), Scott (2005: char. 64*), Pramuk (2006: char. 4), Nussbaum and Wu (2007: char. 52*), Mendelson et al. (2011: char. 25*).

7. Nasals, medial contact: $(0)$ not in contact medially, (1) in contact medially.
Previous USAGE IN Phylogenetic STUdies: Lynch (1978: char. $\left.2^{\star}\right)$, Clarke (1981: char. $1^{\star}$ ), Ford (1990: char. 1), Morrison (1994: char. $1^{\star}$ ), Báez and Basso (1996: char. 2), Mendelson et al. (2000: char. 32), Scott (2005: char. 63), Fabrezi (2006: char. 1), Pramuk (2006: char. 3), Nussbaum and Wu (2007: char. 51), Ponssa (2008: char. $52^{\star}$ ).

8. Contact between nasal and frontoparietal: (0) anterior margin of frontoparietal does not articulate with posterior margin of nasal (fig. 2A), (1) articulate only laterally (fig. 2B), (2) articulate along most of its margin but not completely (fig. 2C), (3) articulate along the entire margin (fig. 2D). Additive.

Previous USAGE IN PHYLOGENETIC STUdies: Heyer and Liem (1976: char. $2^{\star}$ ), Morrison (1994: char. $\left.6^{\star}\right)$, Mendelson (1997a: char. $2^{\star}$ ), Pugener et al. (2003: char. 12* [adult morphological characters]), Pramuk (2006: char. $8^{*}$ ), Mendelson et al. (2011: char. $2^{\star}$ ).

9. Dermal roofing bones, sculpturing: $(0)$ dermal bones of the skull completely smooth, (1) lightly exostosed, (2) heavily ornamented with pits, striations, and rugosities. Additive. Hyperossification in anurans involves the level of sculpturing and the number and identity of exostosed bones (see revision by Blotto et al., 2021). Although species of Rhinella display a relatively high diversity of hyperossification, for the time being, we scored the variation only in the dermal roofing bones (nasals and frontoparietal), until more detailed analyses of the skull morphology are carried out.

Previous USAge IN Phylogenetic StudIES: Clarke (1981: char. $2^{\star}$ ), Ford (1990: char. $4^{*}$ ), Morrison (1994: char. 11), Mendelson et al. (2000: char. 28*), Pugener et al. (2003: char. $10^{*}$ [adult morphological characters]), Scott (2005: char. 61*), Fabrezi (2006: char. $2^{\star}$ ), Pramuk (2006: char. 2), Nussbaum and $\mathrm{Wu}$ (2007: char. 62*).

10. Occipital artery pathway, coverage with bone: (0) occipital canal not covered by bone, (1) partially covered, (2) completely covered with bone. Additive. 


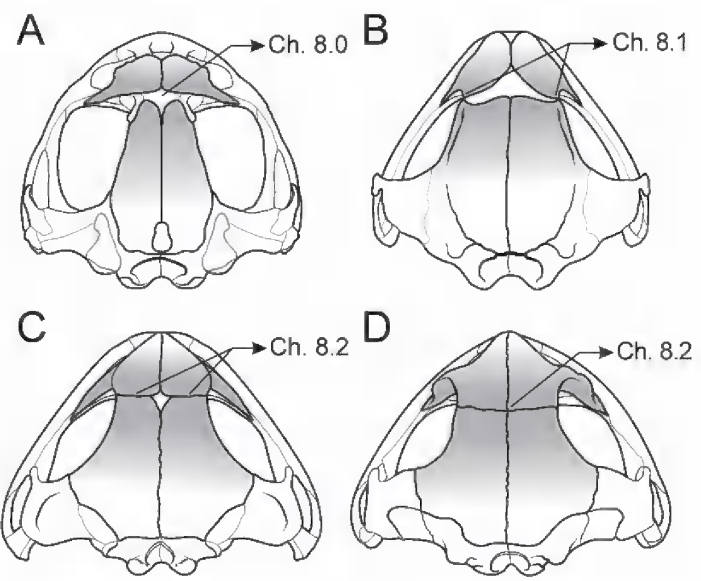

FIG. 2. Skulls (dorsal view) showing the different level of contact between nasals and frontoparietals (both bones in gray): A, Nannophryne cophotis KU 218525 (char. 8.0; species not included in this study); B, Rhinella yanachaga MUSM 24509 (char. 8.1); C, R. crucifer KU 93112 (char. 8.2); D, R. marina KU 152914 (char. 8.3). Panels A, C, D redrawn from Pramuk (2006), B redrawn from Lehr et al. (2007).

Previous usage in Phylogenetic Studies: McDiarmid (1971: char. $7^{*}$ ), Inger (1972: char. $\left.10^{\star}\right)$, Heyer and Liem (1976: char. $\left.3^{\star}\right)$, Lynch (1978: char. $\left.4^{\star}\right)$, Clarke (1981: char. $\left.4^{\star}\right)$, Morrison (1994: char. 10*), Mendelson (1997a: char. $\left.4^{\star}\right)$, Mendelson et al. (2000: char. $\left.38^{\star}\right)$, Pugener et al. (2003: char. 15* [adult morphological characters]), Wiens et al. (2005: char. 16*), Pramuk (2006: char. 9), Mendelson et al. (2011: char. $\left.4^{\star}\right)$.

11. Squamosal, medial extension of otic ramus: (0) otic ramus of squamosal present, but not enlarged, (1) otic ramus of squamosal slightly enlarged, overlapping with the dorsal surface of the crista parotica, (2) otic ramus enlarged, in contact with posterolateral margin of frontoparietal, forming a continuous temporal arcade. Additive.

Previous Usage in Phylogenetic Studies: Inger (1972: char. 12*), Lynch (1978: char. $\left.5^{\star}\right)$, Clarke (1981: char. $5^{\star}$ ), Ford (1990: char. 29*), Báez and Basso (1996: char. 16*), Mendelson (1997a: char. 33*), Faivovich (2002: char. $4^{\star}$ ), Scott (2005: char. 65*), Wiens et al. (2005: char. $\left.15^{\star}\right)$, Fabrezi (2006: char. 10*), Pramuk (2006: char. 15), Nussbaum and Wu (2007: char. $\left.85^{\star}\right)$, Araujo-Vieira et al. (2019: char. 28*).

12. Nasals, extension of anterior margin: (0) anterior margins extend beyond the dorsal mar- gins of the alary processes of the premaxillae (fig. $3 \mathrm{~A}),(1)$ anterior margins are flush with the dorsal margins of the alary processes (fig. 3B), (2) anterior margins lie posterior to the dorsal margins of the alary processes (fig. 3C). Additive.

Previous Usage in Phylogenetic studies: Pramuk (2006: char. 21), Ponssa (2008: char. 57).

13. Premaxilla, orientation of alary process: (0) angled posteriorly to the anterior margin of the premaxillae (fig. 4A), (1) dorsally projected to the anterior margin of the premaxillae (fig. $4 \mathrm{~B}),(2)$ angled anteriorly to the anterior margin of the premaxillae (fig. 4C). Additive.

Previous usage in Phylogenetic studies: Ford (1990: char. 12), Morrison (1994: char. 42), Mendelson (1997a: char. 23), Scott (2005: char. 78), Pramuk (2006: char. 26), Nussbaum and Wu (2007: char. 68), Ponssa (2008: char. 33), Barrionuevo (2017: char. $6^{*}$ ), Araujo-Vieira et al. (2019: char. $\left.19^{*}\right)$.

14. Septomaxilla, level of development of the anterior end: (0) not developed, (1) very developed and exposed anteriorly to the alary process of the premaxilla. Alcalde (2017) showed that bones previously described as "rostrals" (Pregill, 1981) or "prenasals" (Pramuk, 2000, 2006) in some bufonids are actually part of the 

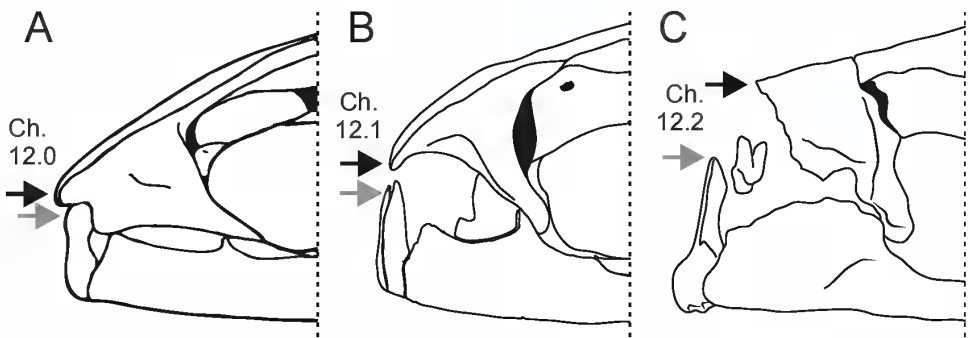

FIG. 3. Skulls (lateral view of the anterior region) showing the relation between the anterior margin of the nasal (black arrow) and the dorsal margin of the alary process of the premaxilla (gray arrow): A, Rhinella yanachaga MSM 24509 (char. 12.0), B, R. amabilis KU 124587 (char. 12.1), C, Schismaderma carens USNM 153380 (char. 12.2). Panels A and B redrawn from Lehr et al. (2007) and Pramuk (2006), respectively. Black arrows indicate the anterior margin of the nasal, gray arrows indicate the dorsal margin of the alary process.

enlarged and exposed anterior ends of the septomaxillae (and thus char. 42 of Pramuk [2006] refers to this structure instead to prenasals bones). Alcalde (2017) also pointed out the presence of an unpaired bone in the anterior end of the snout in Rhinella dorbignyi (as $R$. fernandezae, from the $R$. granulosa Group). He stated that it is homolog to the prenasal bone in some Lophyohylini (Hylidae; Trueb, 1970); even if primary homologs, they clearly represent independent instances of evolution. We observed this element in R. beebei (USNM 566017-8), but we could not determine its occurrence in other species of the group for which we do not consider this bone as a different character (see comments on the preservation and identification of this structure in Alcalde, 2017).

Previous USAGe IN Phylogenetic StUdies: Pramuk (2006: char. $42^{*}$ ).

15. Squamosal, articulation of zygomatic and ventral rami: (0) the zygomatic ramus of the squamosal is free from the ventral ramus, (1) the zygomatic ramus of the squamosal articulates with the ventral ramus of the squamosal.

Previous USAge IN Phylogenetic Studies: Mendelson (1997a: char. 32*), Vélez-Rodriguez (2004b: char. $\left.32^{\star}\right)$, Pramuk (2006: char. $\left.14^{\star}\right)$.

16. Jaw articulation: (0) posterior to the fenestra ovalis, (1) opposite to the fenestra ovalis, (2) anterior to the fenestra ovalis. Additive.

Previous usage in PHYlogenetic Studies: Pramuk (2006: char. 25), Báez et al. (2012: char. 39).
17. Supraorbital flange on the frontoparietals: (0) frontoparietal does not extend laterally beyond the lateral margin of the sphenethmoid, (1) frontoparietal extends laterally beyond the lateral margin of the sphenethmoid.

Previous USAGE IN PHYLOGENETIC STUdies: Morrison (1994: char. 4), Mendelson (1997a: char.1), Mendelson et al. (2000: char. 36), Pugener et al. (2003: char. 13 [adult morphological characters]), Wiens et al. (2005: char. 13), Pramuk (2006: char. 72), Mendelson et al. (2011: char. 1).

18. Sphenethmoid, extent of anterior ossification: (0) bony sphenethmoid reaches the level of palatines, but not beyond, (1) bony sphenethmoid beyond palatines, but does not reach the level of the premaxillae, (2) bony sphenethmoid reaches the level of the premaxillae anteriorly. Additive.

Previous usage in phylogenetic studies: Morrison (1994: char. 20), Mendelson (1997a: char. $\left.13^{\star}\right)$, Vélez-Rodriguez (2004b: char. $21^{\star}$ ), Pramuk (2006: char. $34^{*}$ ), Araujo-Vieira et al. (2019: char. 9*).

19. Pterygoid, articulation of the anterior ramus with maxilla: (0) anterior ramus of pterygoid articulates along the margin of maxilla, but does not contact with the palatine, (1) anterior ramus of pterygoid articulates along the margin of maxilla and contacts the palatine.

Previous usage in Phylogenetic Studies: Ford (1990: char. 32*), Morrison (1994: char. 52), 

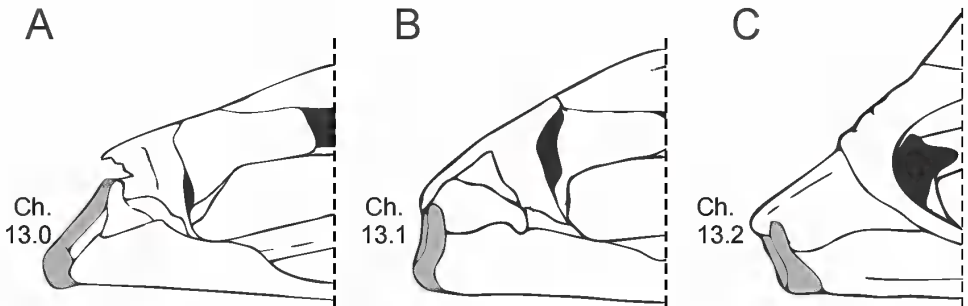

FIG. 4. Skulls (lateral view of the anterior region) showing the orientation of alary process of the premaxilla in relation to the anterior margin of the premaxilla (premaxilla in gray): A, Nannophryne cophotis KU 218525 (char. 13.0; species not included in this study; B, R. crucifer KU 93112 (char. 13.1); C, R. sp. margaritifera Group (char. 13.2). All the figures redrawn and slightly modified from Pramuk (2006). The voucher number provided for the specimen of the $R$. sp. margaritifera Group was erroneously stated in Pramuk's (2006) figures according to the information provided in appendix 1 of that publication and in VertNet database (http://portal.vertnet.org/).

Clarke (1981: char. 13*), Mendelson (1997a: char. $28^{\star}$ ), Pugener et al. (2003: char. $40^{\star}$ [adult morphological characters]), Vélez-Rodríguez (2004b: char. 17*), Ponssa (2008: char. 67), Barrionuevo (2017: char. $\left.29^{\star}\right)$.

20. Palatine, ventral ridge: (0) absent or indistinguishable, (1) present.

Previous USAGe IN Phylogenetic StUdies: Inger (1972: char. 18*), Morrison (1994: char. $\left.33^{\star}\right)$, Mendelson (1997a: char. 15*), Mendelson et al. (2000: char. 10*), Pramuk (2006: char. 38), Mendelson et al. (2011: char. $14^{*}$ ).

21. Pterygoid, contact of medial ramus with ala of parasphenoid: (0) the medial ramus of the pterygoid is not in contact nor fused with the anterolateral margin of the ala of the parasphenoid, (1) the medial ramus of the pterygoid is fused with the anterolateral margin of the parasphenoid, (2) the medial ramus of the pterygoid is fused and extends medially along approximately half the length of the parasphenoid ala. Additive.

Previous Usage IN Phylogenetic Studies: Lynch (1978: char. 9*), Clarke (1981: char. 14*), Ford (1990: char. 34*), Morrison (1994: char. 54), Báez and Basso (1996: char. 28*), Mendelson (1997a: ch 29*), Vélez-Rodríguez (2004b: char. 19*), Pramuk (2006: char. 19).

22. Pterygoid, suture between the medial ramus and parasphenoid alae: (0) the surface of contact is smooth, (1) jagged or scalloped. This character is not applicable for specimens where the medial ramus of the pterygoid is not in contact or not fused with the anterolateral margin of the ala of the parasphenoid (char. 21.0).

Previous USAGE IN PHYLOGENETIC STUdies: Grandison (1981: char. 13*), Pramuk (2006: char. 31).

23. Parasphenoid, shape of anterior margin of cultriform process: ( 0 ) acute and narrow (fig. $5 \mathrm{~A}),(1)$ broadly rounded anteriorly (fig. 5B), (2) truncated (fig. 5C), (3) jagged or scalloped (fig. 5D). Nonadditive.

Previous USAGe in Phylogenetic studies: Clarke (1981: char. 12*), Ford (1990: char. 45*), Morrison (1994: char. 36*), Mendelson (1997a: char. 20*), Scott (2005: char. 54*), Pramuk (2006: char. $\left.29^{\star}\right)$, Nussbaum and Wu (2007: char. 98*), Araujo-Vieira et al. (2019: char. $42^{\star}$ ).

24. Bony protrusion at the angle of jaws: $(0)$ absent or indistinguishable, (1) weak, (2) developed into a processus. Additive. A bony protrusion ("or bony knob") is caused by a variable level of thickening of the ventrolateral margin of the quadratojugal. The level of development of the bony protrusion could also be determined both in living or intact specimens as in osteological preparations.

Previous usage in Phylogenetic StUdies: Vélez-Rodriguez (2004b: char. $36^{\star}$ ).

25. Hyoid, posterior lobe of the anterolateral process: (0) absent or indistinguishable (fig. 6A), (1) present (fig. 6B). 

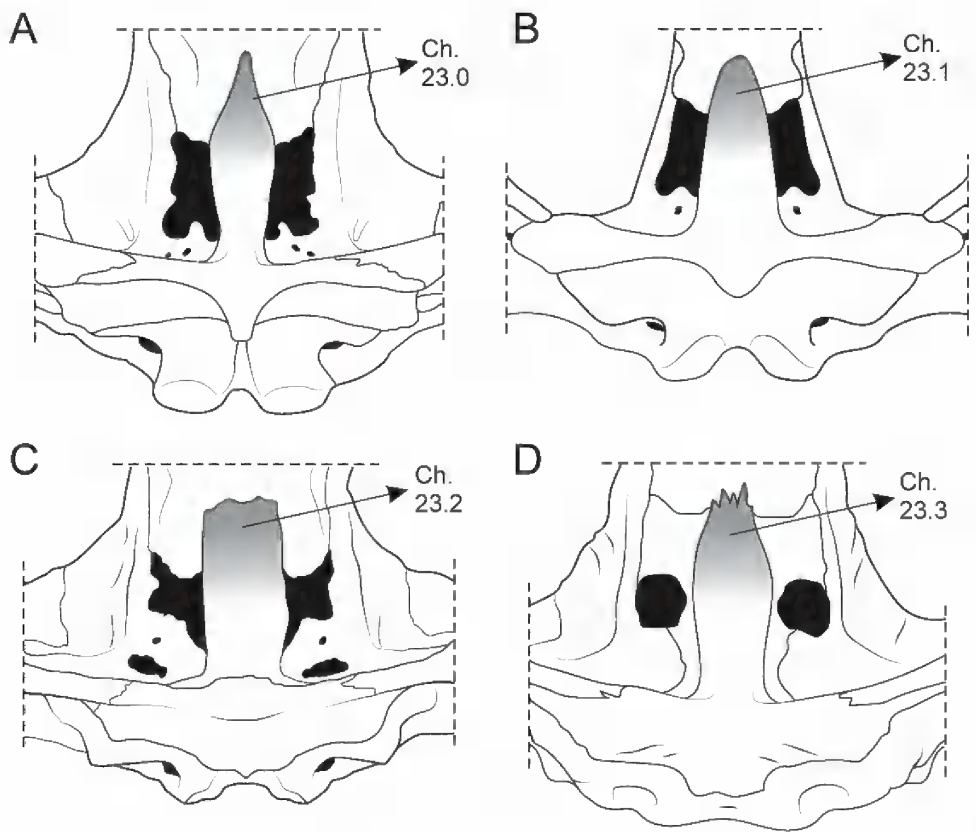

FIG. 5. Skulls (ventral view) showing the anterior margin of cultriform process of the parasphenoid (in gray): A, Rhinella marina KU 152914 (char. 23.0); B, Nannophryne cophotis KU 218525 (char. 23.1); C, R. festae USNM 167168 (char. 23.2); D, R. cristinae ICN 26233 (char. 23.3). Panels redrawn from Pramuk, 2006 (A, B); Trueb, 1971(C) and Vélez-R. and Ruiz-C., 2002 (D).

Previous usage in Phylogenetic Studies: Vélez-Rodriguez (2004: char. 42).

\section{Vertebral Column}

26. Presacral vertebrae, level of development of neural spine: (0) neural spine flat or slightly elevated, (1) neural spine notably elevated, protruding externally. The level of development of the neural spines can be determined both in intact-preserved specimens and in osteological preparations.

Previous USAGe IN PHylogenetic STUdies: Vélez-Rodriguez (2004b: char. 40*).

27. Presacral vertebrae, number: (0) eight, (1) seven. This number refers to the number of vertebrae even if there is some level of fusion between them. The number can be traceable even when there is fusion of centra due to the persistence of the intervertebral foramina (see Trueb, 1973; Cannatella, 1986).
Previous usage in Phylogenetic StudIEs: McDiarmid (1971: char. 23*), Lynch (1973: char. $\left.1^{\star}\right)$, Grandison (1981: char. $\left.15^{\star}\right)$, Cannatella (1986: char. $3^{\star}$ ), Morrison (1994: char. 65*), Báez and Basso (1996: char. 30*), Wiens et al. (2005: char. 51*), Fabrezi (2006: char. 34), Pramuk (2006: char. $44^{*}$ ), Nussbaum and Wu (2007: char. 139), Mendelson et al. (2011: char. 43).

28. Presacral vertebrae I and II, fusion: (0) absent, (1) present. The fusion of the centra of both vertebrae into a single element may be identified for the occurrence of transverse processes and two foramina for vertebral nerves in the anterior presacral element.

Previous Usage in Phylogenetic studies: McDiarmid (1971: char. 24*), Lynch (1973: char. 2), Heyer and Liem (1976: char. 9), Cannatella (1986: char. $4^{\star}$ ), Ford (1990: char. 66), Morrison (1994: char. 66), Wiens et al. (2005: char. 50), Grant et al. (2006: char. $145^{\star}$ ), Nussbaum and 

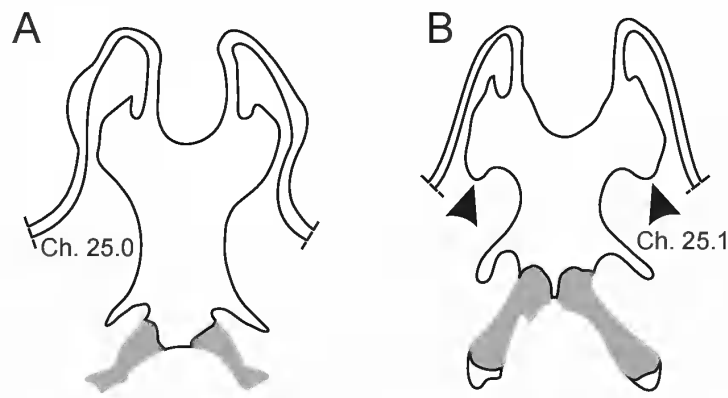

FIG. 6. Hyoid plate: A, Rhinella cristinae ICN 26233 (char. 25.0), B, R. manu MHNC 4404 (char. 25.1). Arrowheads indicate the occurrence of posterior lobes of the anterolateral processes in B. Panels redrawn from Vélez-R. and Ruiz-C., 2002 (A); Chaparro et al., 2007 (B).

Wu (2007: char. 137), Báez et al. (2012: char. 49*), Barrionuevo (2017: char. 43).

29. Sacrum, shape of sacral diapophyses: (0) the maximum width of the sacral diapophysis is smaller than its maximum length, (1) the maximum width of the sacral diapophysis is equal to, or greater than, its maximum length.

Previous USAGE IN PHYLOGENETIC STUdies: Heyer (1975: char. 34*), Heyer and Liem (1976: char. 12*), Ford (1990: char. $75^{\star}$ ), Morrison (1994: char. 70*), Báez and Basso (1996: char. 36*), Faivovich (2002: char. $\left.21^{\star}\right)$, Pugener et al. (2003: char. 57* [adult morphological characters]), Fabrezi (2006: char. $42^{\star}$ ), Grant et al. (2006: char. 143*), Pramuk (2006: char. 51), Araujo-Vieira et al. (2019: char. 95*).

30. Sacrum, orientation of anterior edge of sacral diapophyses: $(0)$ posterior to the midline axis of the vertebral column, (1) perpendicular to the midline axis of the vertebral column, (2) anterior to the midline axis of the vertebral column. Additive.

Previous USAGe in Phylogenetic STUdies: Scott (2005: char. 16), Pramuk (2006: char. 52), Nussbaum and Wu (2007: char. 142).

31. Sacrum and urostyle, fusion: (0) absent, (1) present.

Previous USAGe In Phylogenetic STUdies: McDiarmid (1971: char. 25*), Lynch (1973: char. 3), Ford (1990: char. 76), Pugener et al. (2003: char. 58* [adult morphological characters]), Wiens et al. (2005: char. $60^{*}$ ), Nussbaum and Wu (2007: char. 138), Báez et al. (2012: char. 51*).
32. Ilium, dorsal protuberance, level of development: (0) large and slightly anteriorly or more dorsally directed, (1) small, low, and laterally projected. Gómez and Turazzini (2016) comment on the morphological variation and taxonomic distribution of this structure in anurans.

Previous usage in Phylogenetic studies: Clarke (1981: char. 21*), Morrison (1994: char. 87*), Scott (2005: char. 12*), Pramuk (2006: char. 54), Báez et al. (2012: char. $\left.65^{\star}\right)$.

\section{Adult Musculature}

Foot (ventral surface)

33. Discrete superficial cutaneous tendons, occurrence: (0) absent, (1) present. Burton (2004: 212, 220) described briefly this group of superficial tendons and Blotto et al. (2020) formalized this name. We scored if the superficial tendons are discrete or if they are absent or transformed into a sheet of connective tissue or fascia over the plantar side of the foot. Additional studies are needed to determine whether this group of tendons must be considered as a whole (as here) or individual superficial cutaneous tendons of each digit should be treated as independent characters. See further comments in Blotto et al. (2020).

34. $M$. interphalangeus proximalis digiti $\mathrm{V}$, medial slip, occurrence: (0) absent, (1) present. See Dunlap (1960), Burton (2001, 2004), and Blotto et al. (2020) for descriptions of the $\mathrm{mm}$. 
interphalangei of the foot and comments on its taxonomic distribution in Anura.

35. $M$. interphalangeus proximalis digiti $\mathrm{V}$, lateral slip, occurrence: (0) absent, (1) present.

36. M. abductor brevis plantaris hallucis, occurrence: (0) absent, (1) present. See Burton (2001, 2004) and Blotto et al. (2017) for characterization of this muscle and taxonomic distribution in nonbufonid taxa.

Previous usage in Phylogenetic Studies: Burton (2004: char. 30*), Faivovich et al. (2005: char. 7), Hoyos et al. (2014: char. 44), Blotto et al. (2017: char. 1).

37. M. flexor digiti II (FDM II), position of the origin with respect to the $\mathrm{m}$. intermetatarsalis 1 (IMT 1): (0) FDM II ventral to the IMT 1, (1) FDM II dorsal to the IMT 1, (2) FDM II ventral and dorsal to the IMT 1. Nonadditive. See Dunlap (1960: 42) for an account under the name of $\mathrm{m}$. flexor teres (for the FDM II) and transversus metatarsus (for the $\mathrm{m}$. intermetatarsalis).

38. M. interosseus cruris, presence of an additional origin from the tibiale: $(0)$ absent, (1) present. Most species have both an origin from the tibiale and from the fibulare (Gaupp, 1896; Dunlap, 1960; Burton, 2004). Among bufonid taxa, state 0 was reported for Atelopus (see Dunlap, 1960: 30), under the name of $m$. intertarsalis. The only species from our sampling that has state 0 is Rhinella paraguas.

\section{Foot (dorsal surface)}

39. M. extensor digitorum longus (EDL), insertion on metatarsophalangeal joint of digiti IV: (0) absent, (1) present. We scored the insertion of the EDL in each digit as an independent character, contra Burton (2004: char. 48), as discussed by Faivovich et al. (2005: 201). We found informative variation for the insertions on digits IV and V (next char.). The insertions on the metatarsophalangeal joint of the digits IV and V may be by an independent tendon or through a common tendon with the $\mathrm{m}$. extensor brevis superficialis, $\mathrm{m}$. extensor brevis medius, and/or the $\mathrm{m}$. dorsometatarsalis proximalis, a source of variation not considered in the present study. See Dunlap
(1960) and Burton (2004) for descriptions and variation of the insertion of this muscle, under the name $\mathrm{m}$. extensor digitorum communis longus. The intraspecific variation reported by Inger (1972: 103) for the absence/presence of the insertion on each digit should be further tested; only Nannophryne variegata from our sampling was studied from more than one specimen to test this potential intraspecific variation.

40. M. extensor digitorum longus, insertion on metatarsophalangeal joint of digit $\mathrm{V}$ : (0) absent, (1) present.

Previous USAGe IN PHylogenetiC StUdies: Inger (1972: char. $26^{*}$ ).

41. M. extensor brevis medius hallucis, occurrence: (0) absent, (1) present. See Dunlap (1960: 52-53) for description and variation across Anura.

Previous USAGe in PHylogenetiC StUdies: Hoyos et al. (2014: char. 37).

42. Lateral $\mathrm{m}$. dorsometatarsalis proximalis digiti IV, discrete and independent tendon inserting on the proximal interphalangeal joint of digit IV: (0) absent, (1) present. Dunlap (1960: 57) considered the muscles dorsometatarsales proximales and the dorsometatarsales distales (both as mm. extensores breves profundi) as the same muscle (see discussion in Blotto et al., 2020). This fact partially precludes the understanding of the variation and taxonomic distribution described by Dunlap (1960). On the other hand, the extensive study of Hylidae by Burton (2004: char. H) suggests a great intraspecific variation when considering the number of tendons of insertion of the $\mathrm{mm}$. dorsometatarsales proximales III-V (as extensores breves profundi). In our sampling, all species have a tendon of the lateral $\mathrm{m}$. dorsometatarsalis proximalis digiti IV inserting on the distal interphalangeal joint of digit IV, while Rhinella crucifer and $R$. henseli have an additional independent tendon of insertion on the proximal interphalangeal joint. In the light of the variation found in Bufonidae, as well as in other clades of Anura (B.L.B., personal obs.), we decided to tentatively consider each tendon to each interphalangeal joint as independent transformation series. 
Hand (ventral surface)

43. Medial $\mathrm{m}$. lumbricalis brevis digiti $\mathrm{V}$, slip from distal carpal 3-4-5: (0) absent, (1) present. The medial $\mathrm{m}$. lumbricalis brevis digiti $\mathrm{V}$ may have two slips, one from the distal carpals and the other one from the flexor plate/adjacent tendo superficialis digiti V; both with a common or independent insertions (Burton, 1998: 59; this study). Nevertheless, Burton (1998: char. 18) discarded further discussion and comparison of the nature of this muscle given the extreme degree of variation found within his sampling ("Leptodactylidae" s.l.).

Previous USAGe IN Phylogenetic STUdies: Burton (1998: char.18).

Hand (dorsal surface)

44. M. extensor digitorum, insertion on metacarpophalangeal joint of digiti III: (0) absent, (1) present.

Some species lack the insertion on the metacarpophalangeal joint of the digit III. This insertion may be through a common tendon after inserting on the dorsal fascia of other muscles (usually mm. extensores breves superficiales) or by an independent tendon (Burton, 1998; this study).

Previous USAGe IN Phylogenetic STUdies: Burton (1998: char. 22*).

45. $M$. extensor digitorum, insertion on metacarpophalangeal joint of digiti V: (0) absent, (1) present. The slip of the $\mathrm{m}$. extensor digitorum to the digit $\mathrm{V}$ may have two insertions, one on the metacarpophalangeal joint and a second insertion on the lateral side of the metacarpal $\mathrm{V}$. The presence of both insertions varies independently across Anura (B.L.B., personal obs.), for which we scored their presence as independent transformation series. Within the current sampling of Bufonidae, the lateral insertion on metacarpal $\mathrm{V}$ is invariably present, and thus variation is restricted to the presence of the insertion on the metacarpophalangeal joint. This insertion may be through a common tendon after insertion on the dorsal fascia of other muscles or by an independent tendon (Burton, 1998; Araujo-Vieira et al., 2019; this study).
Previous USAge in Phylogenetic StUdies: Araujo-Vieira et al. (2019: char. 171).

46. M. extensor carpi ulnaris, occurrence of a head from the radioulna: (0) absent, (1) present. This head was not previously reported in the literature. It originates from the distal half or quarter of the radioulna, laterally to the origin of the $\mathrm{m}$. abductor pollicis longus. The head converges with the head from the humerus, which attaches to the ulnare and distal carpal 3-4-5 (fig. 7).

47. M. extensor carpi ulnaris, nature of the origin of the head from the radioulna: (0) fleshy (fig. 7B), (1) via a flat tendon (fig. 7D). This character is not applicable for specimens that lack a supplementary head from the radioulna (char. 46.0).

\section{Tympanic Middle Ear Complex}

Pereyra et al. (2016b) reported the range of variation in structures of the tympanic middle ear (i.e., columella, annulus tympanicus, and tympanic membrane) in Bufonidae and demonstrated its unique evolutionary pattern within Anura.

48. Columella, occurrence: (0) absent, (1) present.

Previous usage in Phylogenetic studies: Grandison (1981: char. $1^{\star}$ ), Cannatella (1986: char. $6^{*}$ ), Ford (1990: char. 11), Morrison (1994: char. 27), Mendelson (1997a: char. 38*), Pugener et al. (2003: char. $47^{\star}$ [adult morphological characters]), Scott (2005: char. 81), Pramuk (2006: char. 17), Nussbaum and $\mathrm{Wu}$ (2007: char. $\left.67^{\star}\right)$, Mendelson et al. (2011: char. 27).

49. Annulus tympanicus, occurrence: (0) absent, (1) present.

Previous USAGe IN Phylogenetic STUdies: Inger (1972: char. 28*), Drewes (1984: char. $23^{\star}$ ), Cannatella (1986: char. $\left.8^{\star}\right)$, Scott (2005: char. $80^{\star}$ ), Wiens et al. (2005: char. $\left.35^{\star}\right)$, Nussbaum and Wu (2007: char. 66).

50. Tympanic membrane: (0) absent, (1) present.

Previous USAGE IN PHylogenetic STUdies: Inger (1972: char. 28*), Heyer (1975: char. $2^{\star}$ ), Drewes (1984: char. 23*), Cannatella (1986: char. 

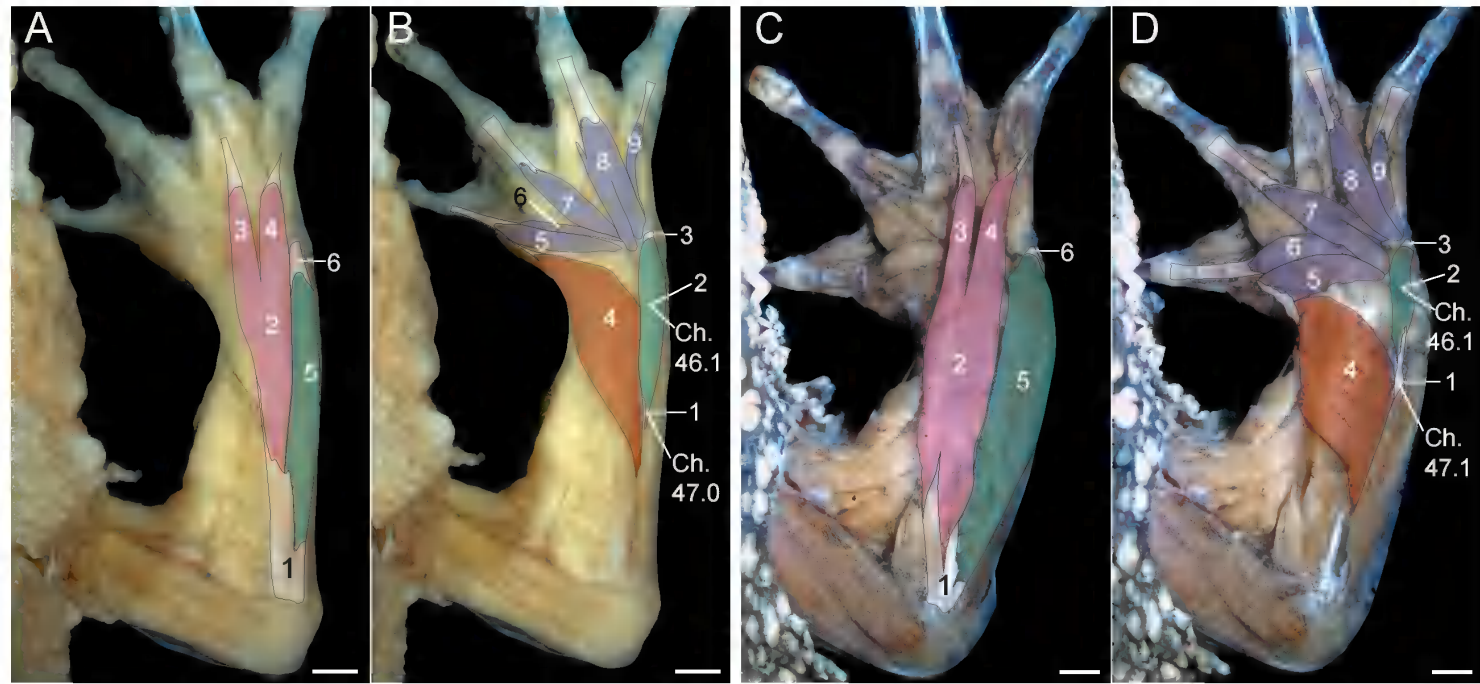

FIG. 7. Musculature of the dorsal surface of the hand and forearm: A, B. Rhinella paraguas CD 870. C, D. R. dorbignyi MACN 39350. A. First muscular layer: $\mathrm{m}$. extensor digitorum; the $\mathrm{m}$. extensor carpi ulnaris is also shown. Elements figured: 1, m. extensor digitorum and the $\mathrm{m}$. extensor carpi ulnaris (head from humerus): common tendon of origin; $2, \mathrm{~m}$. extensor digitorum; $3, \mathrm{~m}$. extensor digitorum: slip to the dorsal surface of the $\mathrm{m}$. extensor brevis superficialis digiti IV (both muscles attaches to the metacarpophalangeal joint of digit IV via a common tendon); 4, m. extensor digitorum: slip to metacarpal V; $5, \mathrm{~m}$. extensor carpi ulnaris: head from humerus; 6, m. extensor carpi ulnaris (head from humerus): tendon of insertion. B. Second muscular layer: $\mathrm{m}$. abductor pollicis longus and $\mathrm{mm}$. extensores breves superficiales; the head from radioulna of the $\mathrm{m}$. extensor carpi ulnaris is also shown (the head from humerus was removed). Elements figured: $\mathbf{1}, \mathrm{m}$. extensor carpi ulnaris (head from radioulna): fleshy origin; 2, m. extensor carpi ulnaris: head from radioula; 3, $\mathrm{m}$. extensor carpi ulnaris (heads from humerus and radioulna): common tendon of insertion on distal carpal $3-4-5 ; \mathbf{4}, \mathrm{m}$. abductor pollicis longus; $\mathbf{5}, \mathrm{m}$. extensor indicis brevis superficialis: slip from radiale; $\mathbf{6}, \mathrm{m}$. extensor indicis brevis superficialis: slip from ulnare; $7, \mathrm{~m}$. extensor brevis superficialis digiti III; $\mathbf{8}, \mathrm{m}$. extensor brevis superficialis digiti IV: slips from ulnare and distal carpal 3-4-5; 9, m. extensor brevis superficialis digiti V. C. First muscular layer: $\mathrm{m}$. extensor digitorum; the $\mathrm{m}$. extensor carpi ulnaris is also shown. Elements figured: 1, m. extensor digitorum and $\mathrm{m}$. extensor carpi ulnaris (head from humerus): common tendon of origin; 2, m. extensor digitorum; 3, m. extensor digitorum: slip to the dorsal surface of the $\mathrm{m}$. extensor brevis superficialis digiti IV (both muscles attaches to the metacarpophalangeal joint of digit IV via a common tendon); 4, m. extensor digitorum: slip to metacarpal V; 5, m. extensor carpi ulnaris: head from humerus; 6, m. extensor carpi ulnaris: tendon of insertion. D. Second muscular layer: $\mathrm{m}$. abductor pollicis longus and $\mathrm{mm}$. extensores breves superficiales; the head from radioulna of the $\mathrm{m}$. extensor carpi ulnaris is also shown (the head from humerus was removed). Elements figured: 1, m. extensor carpi ulnaris (head from radioulna): tendon of origin; 2, m. extensor carpi ulnaris: head from radioulna; 3, m. extensor carpi ulnaris (heads from humerus and radioulna): common tendon of insertion on distal carpal 3-4-5; 4, m. abductor pollicis longus; $5, \mathrm{~m}$. extensor indicis brevis superficialis: slip from ulnare inserting on metacarpal II (in common with the m. abductor pollicis longus); 6, m. extensor indicis brevis superficialis: slip from ulnare inserting on metacarpophalangeal joint; 7, m. extensor brevis superficialis digiti III: slips from ulnare and distal carpal 3-4-5; 8, m. extensor brevis superficialis digiti IV: slip from distal carpal 3-4-5; 9, m. extensor brevis superficialis digiti V. Characters figured: char. 46.1, presence of the head from radioulna of the m. extensor carpi ulnaris; char. 47.0, fleshy origin of the head from radioulna of the m. extensor carpi ulnaris; char. 47.1, origin via a flat tendon of the head from radioulna of the $\mathrm{m}$. extensor carpi ulnaris. Scale bars $=1 \mathrm{~mm}$. 
$8^{\star}$ ), Morrison (1994: char. 96*), Scott (2005: char. $\left.144^{*}\right)$, Wiens et al. (2005: char. $\left.108^{\star}\right)$, Ohler and Dubois (2006: char. $\left.4^{\star}\right)$, Nussbaum and Wu (2007: char. 4), Barrionuevo (2017: char. 60).

\section{Adult Visceral Anatomy}

51. Inguinal fat bodies, occurrence: (0) absent, (1) present. Boulenger (1910) first reported the occurrence of elongated bodies associated to the muscles of the inguinal region in several species of Bufonidae. Later, Plytycz and Szarski (1987) and da Silva and Mendelson (1999) corroborated the occurrence of these inguinal fat bodies in many other species of several bufonid genera.

Previous USAGe IN Phylogenetic STUdies: Mendelson (1997a: char. 45), Pramuk (2006: char. 79), Mendelson et al. (2011: char. 34).

\section{Adult External Morphology}

All described characters of external morphology refer to adult individuals of both sexes, except when sexually dimorphic characters are considered (i.e., chars. 53-54 and 58-59).

52. Dorsal skin, macroscopic glandular structures in females: (0) indistinct; (1) small and smooth; (2) greatly enlarged and smooth; (3) with small tubercles, without cornified tip; (4) conical with a single cornified tip; (5) hemispherical with multiple cornified tips. Nonadditive. The skin of bufonids varies from completely smooth to highly tuberculated and warty due to modifications of dermal and/or epidermal components (Elias and Shapiro, 1959). It differs between sexes and its structure is affected (at least in males) by the reproductive condition of the specimens (see Cei, 1980; Duellman and Trueb, 1986). Therefore, when scoring skin diversity we considered: (1) as independent character structures present in both sexes (chars. 53, 54); (2) the maximum level of development reported for the dorsal structures of skin within the studied specimens; and (3) the most common structures present in the dorsal skin. Although some character states seem to be com- posed of a progressive series of transformation of glandular structures, detailed histological studies are required to understand the various components that are differentiated in each structure.

Previous usage in Phylogenetic studies: McDiarmid (1971: char. 39*), Morrison (1994: char. 99*), Grant et al. (2006: char. $\left.0^{\star}\right)$.

53. Dorsal skin, macroscopic glandular structures in males: (0) indistinct; (1) small and smooth; (2) greatly enlarged and smooth; (3) with small tubercles, without a cornified point; (4) conical with a single cornified point; (5) hemispherical with multiple cornified points. Nonadditive.

Previous USAGe in Phylogenetic StUdies: McDiarmid (1971: char. 39*), Morrison (1994: char. 99*), Grant et al. (2006: char. $\left.0^{*}\right)$.

54. Vertebral line, occurrence: (0) absent, (1) present. Boulenger (1897) first noted the distinctiveness and independence between a filiform line or raphe and the vertebral line. The former is a line of thin skin extending along the middle of the back from the snout to the vent. This raphe is very common in bufonids and gives rise to a light vertebral line. As pointed out by Boulenger (1897), the independence of these structures is evident in cases of deviation of the former (see Boulenger, 1897: fig. 9). We consider the occurrence of a distinctive light vertebral line only.

Previous USAGE IN PHYLOGENETIC STUDIES: Heyer (1978: char. $\left.1^{\star}\right)$, Ohler and Dubois (2006: char. $13^{\star}$ ), Ponssa (2008: char. $\left.1^{\star}\right)$.

55. Parotoid gland, occurrence: $(0)$ absent, (1) present.

Previous USAGe in PHylogenetic StUdies: Cannatella (1986: char. 11), Morrison (1994: char. 95*), Mendelson (1997a: char. 43*), Wiens et al. (2005: char. 109), Fabrezi (2006: char. 72), Pramuk (2006: char. $73^{\star}$ ).

56. Parotoid gland, shape: $(0)$ approximately ellipsoid, longer than wide (fig. 8A); (1) subtriangular (fig. 8B); (2) round to ovoid mostly symmetrical (fig. 8C); (3) triangular and bulky (fig. $8 D)$. Nonadditive. This character is not applicable for specimens that lack a parotoid gland (char. 55.0). 
A

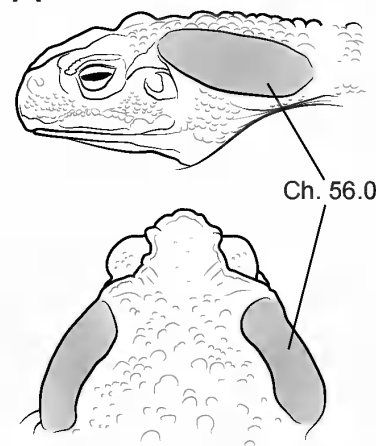

B

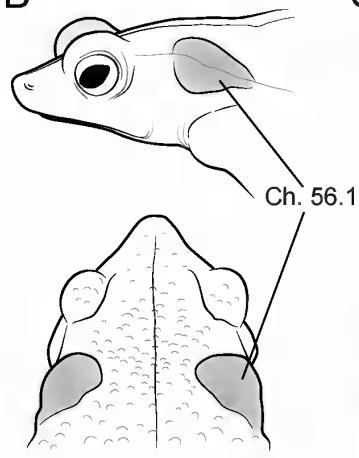

C
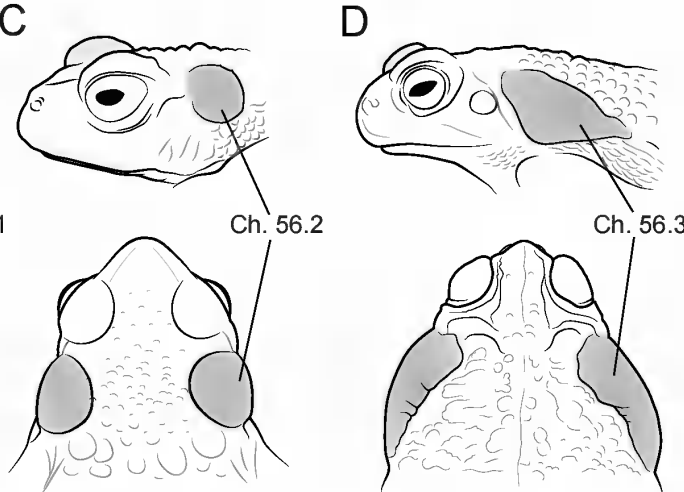

FIG. 8. Head (lateral and dorsal views) showing the shape of the parotoid gland (in gray): A, Rhinella aff. cerradensis (char. 56.0); B, R. acutirostris (char. 56.1); C, R. arunco (char. 56.2); D, R. marina (char. 56.3).

Previous usage in Phylogenetic Studies: Morrison (1994: char. 95*), Pramuk (2006: char. $73^{\star}$ ), Mendelson et al. (2011: char. 32*).

57. Skin, occurrence of row of dorsolateral tubercles: (0) absent, (1) present.

Previous USAGe in Phylogenetic Studies: Morrison (1994: char. 97), Mendelson (1997a: char. $44^{\star}$ ), Pramuk (2006: char. 81), Mendelson et al. (2011: char. 33).

58. Vocal sac, occurrence in adult males: $(0)$ absent, (1) present. The vocal sac develops as ventral diverticula of the mouth floor into spaces among submandibular muscles (Noble, 1931; Tyler, 1971). This second cavity communicates with the buccal cavity via single or paired apertures, the vocal slits. In this way, the presence of a vocal sac automatically implies the presence of at least one vocal slit (and vice versa). Modifications of the gular skin (i.e. "internal" or "external" vocal sacs sensu Günther, 1858a) can be absent or present without affecting the codification of this character. Vocal sacs are either absent or present in adult males of the majority of species, with few exceptions where both states cooccur in different specimens (Liu, 1935; Inger and Greenberg, 1956, Hayes and Krempels, 1986; Mendelson, 1997b).

Previous USAGe IN Phylogenetic Studies: Liem (1970: char. 36*), Drewes (1984: char. 20*), Cannatella (1986: char. 10*), Hillis and de Sá (1988: char. 6), Mendelson et al. (2000: char.
51*), Grant et al. (2006: char. $\left.76^{\star}\right)$, Pramuk (2006: char. $\left.75^{\star}\right)$, Ohler and Dubois (2006: char. 14), Mendelson et al. (2011: char. $\left.31^{\star}\right)$.

59. Vocal slits, number: (0) unilateral, (1) bilateral. Several authors (e.g., Boulenger, 1897; Liu, 1935; Inger and Greenberg, 1956) reported the occurrence of specimens with a single vocal slit. This condition was observed in some species of Bufonidae and has not been reported in other anuran families. The single vocal slit can either be on the left or the right side of the tongue in different specimens of the same species. Furthermore, there are species where one (on either side) or two vocal slits can occur. This character is scored as not applicable for taxa lacking vocal sacs (see char. 58.0).

Previous USAGE IN PHYlogenetic STUdies: Drewes (1984: char. 20*), Cannatella (1986: char. $10^{*}$ ), Mendelson (1997a: char. 42*), Mendelson et al. (2000: char. 51*), Pramuk (2006: char. $\left.75^{\star}\right)$, Mendelson et al. (2011: char. 31*).

60. Vocal sac, shape when fully inflated: (0) spherical or subspherical, (1) projected anteriorly. Simple subgular vocal sacs are often spherical or subspherical. Nevertheless, in a few species, they project anteriorly deviating from a spherical shape. The degree of projection ranges from a slight deformation to a large, vertically oriented lobe. McAllister (1961) reported on this variation in North American bufonids and their putative 
relationship with vocalization, but this character has not been used in phylogenetic studies. This character is not applicable for specimens that lack a vocal sac (char. 58.0)

61. Nuptial pads, occurrence in males: $(0)$ absent, (1) present. Nuptial pads are sexually dimorphic structures that can be present in the fingers of males; their structure and diversity were recently studied (Luna et al., 2018).

Previous usage in Phylogenetic STUdies: Liem (1970: char. 35), Heyer (1975: char. $3^{*}$ ), Scott (2005: char. 132*), Wiens et al. (2005: char. 100), Grant et al. (2006: char. 23), Ohler and Dubois (2006: chars. 16-18*), Ponssa (2008: char. $24^{*}$ ), Barrionuevo (2017: char. 69).

62. Nuptial pads, coloration: (0) light colored, (1) dark colored. Following Luna et al. (2018) we distinguished between dark- and lightcolored nuptial pads, where "dark-colored" includes all tones of brown and black and "lightcolored" includes beige/uncolored pads. These differences in coloration result from minor changes in the stratum corneum of the epidermis and are independent of the number of layers of this stratum (Luna et al., 2008). This character is not applicable for specimens that lack nuptial pads (char. 61.0).

Previous USAGe IN Phylogenetic STUdies: Ohler and Dubois (2006: char. $24^{\star}$ ).

63. Manus, occurrence of webbing between fingers: (0) absent or poorly developed, (1) present, well developed.

Previous Usage in Phylogenetic Studies: Wiens et al. (2005: char. 99), Pramuk (2006: char. $\left.77^{\star}\right)$, Nussbaum and Wu (2007: char. 12*).

64. Pes, edge of foot webbing: (0) smooth, (1) serrated.

Previous USAGE IN PHYLOGENETIC STUdies: Vélez-Rodriguez (2004b: char. $12^{\star}$ ).

65. Tarsus, occurrence of tarsal fold: (0) absent, (1) present. A tarsal fold is a dermal fold on the medial-ventral surface of the foot, extending proximally from the inner metatarsal tubercle.

Previous USAge IN Phylogenetic STUdIES: Inger (1972: char. 31*), Heyer (1975: char. $\left.6^{*}\right)$, Scott (2005: char. 156*), Grant et al. (2006: char. 28), Ohler and Dubois (2006: char. $11^{*}$ ), Ponssa (2008: char. 19), Barrionuevo (2017: char. 77).

66. Relative size of adult females and males: (0) adult females similar in size or larger than adult males, (1) adult males much larger than adult females. As a first approximation, we consider only two states due to the occurrence of a more evident gap in size according to published data. However, a more detailed study of sexual dimorphism in Rhinella could help to partition these into more additional states.

Previous USAGe In Phylogenetic StUdies: Scott (2005: char. 139), Fabrezi (2006: char. $\left.78^{\star}\right)$, Ponssa (2008: char. $110^{\star}$ ).

\section{LaRval External Morphology}

67. Body, morphology of the peribranchial and abdominal regions: (0) absence of external modifications, (1) presence of bulging regions lateral to the oral disc, (2) occurrence of an abdominal sucker. Additive. Most species of Rhinella have lentic larvae that lack external modifications in the peribranchial and abdominal regions (state $0)$. Modifications in these regions are typical of some lotic forms (McDiarmid and Altig, 1999; Hoff et al., 1999) and two different states occur within Rhinella. Larvae of Rhinella rumbolli have a central depression delimited by bulbous lateral regions in the peribranchial zone (state 1). Moreover, some other species of the $R$. veraguensis Group have a well-developed abdominal sucker that is bounded anteriorly by the oral disc, and the lateral and posterior edges are free from the body (state 2). We consider the character states to represent an ordered series of transformation for which the states are considered as additive.

68. Body, dorsal coloration: (0) light brown, (1) dark brown, (2) sharply defined dark markings on pale ground. Nonadditive.

69. Caudal musculature, ocurrence of an unpigmented longitudinal stripe along the inferior edge in the caudal musculature: (0) absent, (1) present. An unpigmented longitudinal stripe 
along the inferior edge of the caudal musculature sometimes occur in the caudal musculature of larvae having a dark coloration of the tail.

Previous USAGe IN PHylogenetic STUdies: Mendelson et al. (2011: char. $38^{*}$ ).

70. Caudal musculature, occurrence of irregular transverse whitish stripes: $(0)$ absent, (1) present. In some species of the Rhinella granulosa and $R$. veraguensis Groups there are irregular transverse whitish stripes of variable extension due to the absence of melanocytes contrasting with the general dark coloration of the dorsal musculature (see Blotto et al., 2014, for taxonomic distribution in Rhinella).

71. Oral disc, occurrence of submarginal papillae: (0) absent, (1) present.

Previous usage in Phylogenetic studies: Grant et al. (2006: char. 91*), Barrionuevo (2017: char. 86*), Araujo-Vieira et al., (2019: char. 135).

72. Oral disc, number of posterior labial tooth rows: $(0)$ two, (1) three.

Previous USAge IN PHYlogenetic STUdies: Hillis and de Sá (1988: char. $\left.2^{\star}\right)$, Wiens et al. (2005: char. 122*), Grant et al. (2006: char. 94*), Ohler and Dubois (2006: char. $31^{\star}$ ), Barrionuevo (2017: char. 90*), Araujo-Vieira et al. (2019: char. 141*).

73. Oral disc, condition of the labial tooth row A2: (0) complete, (1) divided.

Previous USAGE IN PHYLOGENETIC STUdies: Mendelson et al. (2011: char. $\left.37^{\star}\right)$.

74. Oral disc, condition of the labial tooth row P1: (0) complete, (1) divided.

Previous USAGe IN Phylogenetic Studies: Wiens et al. (2005: char. 124), Araujo-Vieira et al. (2019: char. 142)

75. Vent tube, opening: (0) medial, (1) dextral.

Previous usage in Phylogenetic studies: Grant et al. (2006: char. 96*), Barrionuevo (2017: char. 93), Araujo-Vieira et al., 2019 (char. 145).

\section{LARVAL CHONDROCRANIUM}

Oliveira et al. (2014) studied the chondrocranium of some species of Rhinella and reviewed the information available for other bufonids.
76. Otic capsule, larval crista parotica, occurrence of processus anterolateralis: (0) absent or indistinguishable, (1) poorly developed with a rounded aspect, (2) well developed with an acute appearance. Additive.

Previous USAge in Phylogenetic StUdies: Larson and de Sá (1998: char. $\left.j^{\star}\right)$, Haas (2003: char. $\left.66^{\star}\right)$; Miranda et al. (2015: char. 61*).

77. Procesus ascendens, angle of attaching to the braincase: $(0)$ obliquely attached, (1) perpendicularly attached.

Previous USAGe In Phylogenetic STUdies: Larson and de Sá (1998: char. $\left.0^{*}\right)$, Miranda et al. (2015: char. 65*).

78. Copula anterior, occurrence: $(0)$ absent, (1) present.

Previous usage in Phylogenetic Studies: Haas (2003: char. 105), Pugener et al. (2003: char. 35 [larval characters]), Miranda et al. (2015: char. 73).

\section{EMBRYONIC MORPHOLOGY}

Vera Candioti et al. (2016) studied the early ontogeny and described the informative variation found in several species of Bufonidae. All the characters considered on embryonic morphology were described in detail in that publication.

79. Third pair of external gills, condition: (0) absent or indistinguishable, (1) short, (2) long. Additive.

80. Dorsal line of hatching glands: $(0)$ short (cephalic region only), (1) long (beyond cephalic region).

81. Type of adhesive gland: (0) A, (1) B.

82. Time of division of adhesive gland: $(0)$ slightly after the second-gill pair branches off before operculum at the gill base, (1) immediately before the gills reach their maximum development, (2) immediately after opercular fusion. Additive.

\section{Natural History}

83. Diel activity of adults: (0) diurnal, (1) nocturnal. 
Previous USAGe IN Phylogenetic StUdies: Grant et al. (2006: char. $\left.115^{*}\right)$.

84. Habits: (0) terrestrial, (1) arboreal, (2) aquatic. Nonadditive. Some species of the Rhinella veraguensis Group are completely arboreal. We do not consider as arboreal the mostly terrestrial species that have the ability to climb up the vegetation to rest during the night (de Noronha et al., 2013).

Previous USAGe IN PHYLOGENETIC STUdies: Grant et al. (2006: char. $\left.114^{*}\right)$.

85. Oviposition site: (0) aquatic, (1) terrestrial, (2) phytotelmata. Nonadditive. Following van Bocxlaer et al. (2010), terrestrial oviposition refers to eggs that are placed on the ground, in leaf litter, or under stones, and are exposed to little or no free water at the time of oviposition. Phytotelmata refers to any chambers in a plant that is used as oviposition site (e.g., water-filled nut, tree holes, leaf axils; see Lehtinen et al., 2004; Grant et al., 2006).

Previous USAGe IN Phylogenetic STUdies: Faivovich (2002: char. 83*), Grant et al. (2006: char. 107*), Araujo-Vieira et al. (2019: char. $\left.191^{*}\right)$.

86. Structure of the spawn: (0) strings, (1) open clump, (2) mass, (3) strands. Nonadditive. Altig and McDiarmid (2007) reviewed in detail the terminology and diversity of arrangement of deposited eggs in Amphibia.

Previous USAGE IN PHYLOGENETIC STUDIES: Haas (2003: char. 141*).

87. Egg disposition in strings: (0) uniserial, (1) biserial, (2) multiserial. Nonadditive. Mature oocytes are surrounded by jelly layers as they are displaced through the different regions of the oviduct (Salthe, 1963; Altig and McDiarmid, 2007). The number and type of jelly layers are not well characterized in Rhinella (Pereyra et al., 2015), and there is no information about a direct relation between the diversity of strings and the eggs disposition within the string. Thus, we cannot infer a series of transformation and we consider this character as nonadditive.

88. Ovum pigmentation: (0) unpigmented, (1) animal pole pigmented.
Previous USAGe in Phylogenetic StUdies: McDiarmid (1971: char. 42*), Grandison (1981: char. $4^{\star}$ ), Cannatella (1986: char. $\left.14^{\star}\right)$, Grant et al. (2006: char. 68*), Ohler and Dubois (2006: char. 29), Mendelson et al. (2011: char. 40*).

\section{Cytogenetics}

89. Nucleolar Organizer Regions, location: (0) terminal position of the short arms of the chromosome pair 1, (1) pericentromeric position of the long arms of the chromosome pair 1, (2) terminal position of the long arms of the chromosome pair 5, (3) terminal position of the long arms of the chromosome pair 6 , (4) interstitial position of the short arms of the chromosome pair 7, (5) interstitial position of the long arms of the chromosome pair $10,(6)$ terminal position of the long arms of the chromosome pair 10, (7) interstitial position of the short arms of the chromosome pair 11. Nonadditive.

Previous USAGe IN Phylogenetic StUdies: Faivovich (2002: char. $82^{\star}$ ).

\section{RESULTS}

Separate Analyses of Restricted Nuclear (RND) AND MitochondRial (RMD) Datasets

The parsimony analyses, reaching a stable consensus 10 times, retained 706 unique MPTs of length 1757 for the rND and one MPTs of length 11,436 for the rMD. Within the ingroup (i.e., Rhinella), the main incongruence between the rND and rMD analyses involved the position of the specimens of $R$. horribilis, which are deeply nested within the $R$. marina Group in the rND analysis, but were recovered as the sister clade of the $R$. marina $+R$. crucifer Groups in the rMD analysis (fig. 9). Based on these observations and previous published results (Pereyra et al., 2016a), we included the mitochondrial and nuclear genomes of $R$. bernardoi and $R$. horribilis as independent terminals in the TE analysis (see Discussion section for comments on the putative mitochondrial or nuclear introgression in these terminals 
and the rationale for the considerations of both genomes as independent terminals). Mitochondrial introgression and hybridization between $R$. diptycha and $R$. marina might have occured in the area south of the Amazon River (see Sequeira et al., 2011), but the evidence is not conclusive (see Vallinoto et al., 2017). For this reason, we did not include sequences of these species from this complex area. In appendix 2, we list the terminals considered in the TE analysis.

\section{Total Evidence Analysis}

Molecular data were included for all 320 terminals of 124 species, whereas phenotypic data were restricted to 106 specimens of 102 species (90 characters; $~ 50$ scores/terminal). The TE analysis using parsimony, reaching a stable consensus 10 times, retained 657 unique MPTs (length 25,399). One of the optimal topologies is shown in figures 10-14 (fig. 10 for outgroup relationships, figs. 11-14 for Rhinella relationships). A summary tree of Rhinella relationships to species level is shown in the supplementary data 2 (available at https:// doi.org/10.5531/sd.sp.46). In depicting all unrefuted clades, we employ the strict consensus of the optimal phylogenetic hypotheses resulting from this TE analysis treating gaps as fifth state as the basis of our discussion of taxonomy. The results of the TE analysis considering gaps as missing data (see supplementary data 3.1-3.5, available at https://doi.org/10.5531/sd.sp.46) and the ML analysis (see supplementary data 4.1-4.5, available at https://doi.org/10.5531/sd.sp.46) were highly congruent with the TE analysis considering gaps as fifth state. The few differences between these hypotheses are discussed when relevant.

The MPTs resulting from the TE analysis recovered Rhinella as nonmonophyletic due to the position of $R$. ceratophrys that is the sister taxon of Rhaebo nasicus with strong support (JGC and $\mathrm{JAF}=100 \%$; see fig. 10). Among outgroups the strongly supported Anaxyrus + Incilius (JGC = $96 \%$, JAF $=97 \%$ ) is the sister clade of all the other species of Rhinella. The monophyly of the clade composed of these three genera is poorly supported $(\mathrm{JGC}=63 \%$, JAF $=73 \%)$. The species of Rhinella (excluding R. ceratophrys) are monophyletic, well supported (JGC and JAF $=98 \%$ ), and grouped in two major clades. One of these is moderately supported $(\mathrm{JGC}=88 \%, \mathrm{JAF}=92 \%$ ) and includes the species of the former $R$. spinulosa Group (including R. gallardoi; see Discussion) and those of the R. granulosa, R. crucifer, and $R$. marina Groups (figs. 11, 12). The other is strongly supported (JGC and JAF $=99 \%$ ) and composed of all the species from the nonmonophyletic $R$. veraguensis and $R$. margaritifera Groups, the former $R$. acrolopha Group (see Discussion section), and $R$. sternosignata (figs. 13, 14).

\section{UnCORRECTED P-Distances}

The patterns of UPDs found within each species group vary largely (see below), so we did not consider a single value as a threshold to delimit species, but each particular situation was considered in the context of the genetic distances found within each species group. Interspecific distances among all the species addressed by the taxonomic revision are presented in the Discussion section of each species group. Throughout the text the UPDs are expressed as percentage.

\section{DISCUSSION}

\section{Systematics and Taxonomy}

\section{Relationships Among OUtgroups AND RHINELLA}

Our outgroup sample was designed exclusively to provide a rigorous test of the monophyly of $R h i$ nella and does not constitute a critical test of previously hypothesized relationships among other clades of Bufonidae (e.g., Frost et al., 2006; Pramuk, 2006; Pramuk et al., 2008; van Bocxlaer et al., 2010; Pyron and Wiens, 2011; Pyron, 2014; Portik and Papenfuss, 2015; Jetz and Pyron, 2018). Indeed, most of the basal relationships of Bufonidae are unresolved or poorly supported in the TE analysis (fig. 10). Nevertheless, we found Anaxyrus + Incilius to be the sister clade of Rhinella with low support 
rND

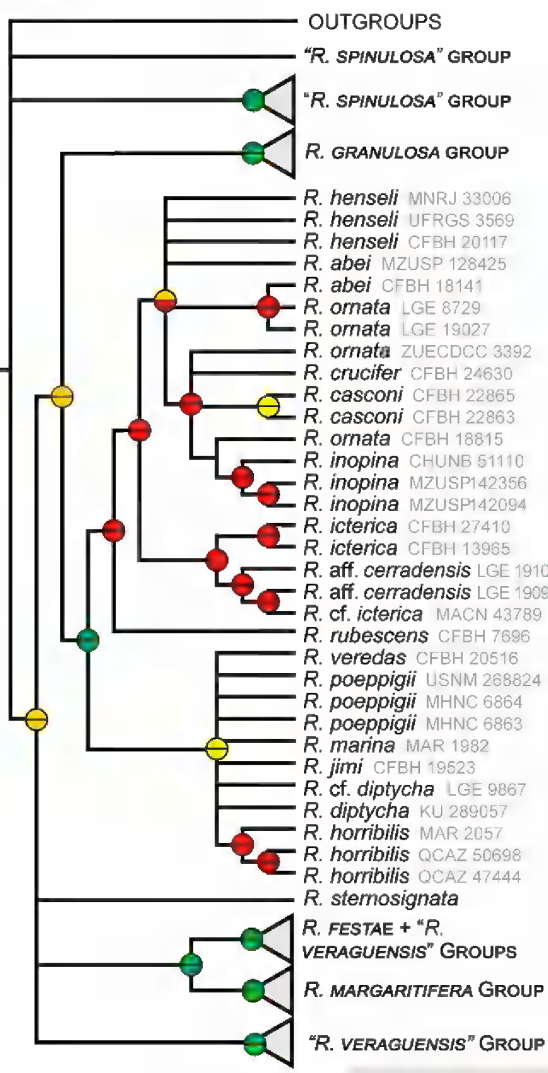

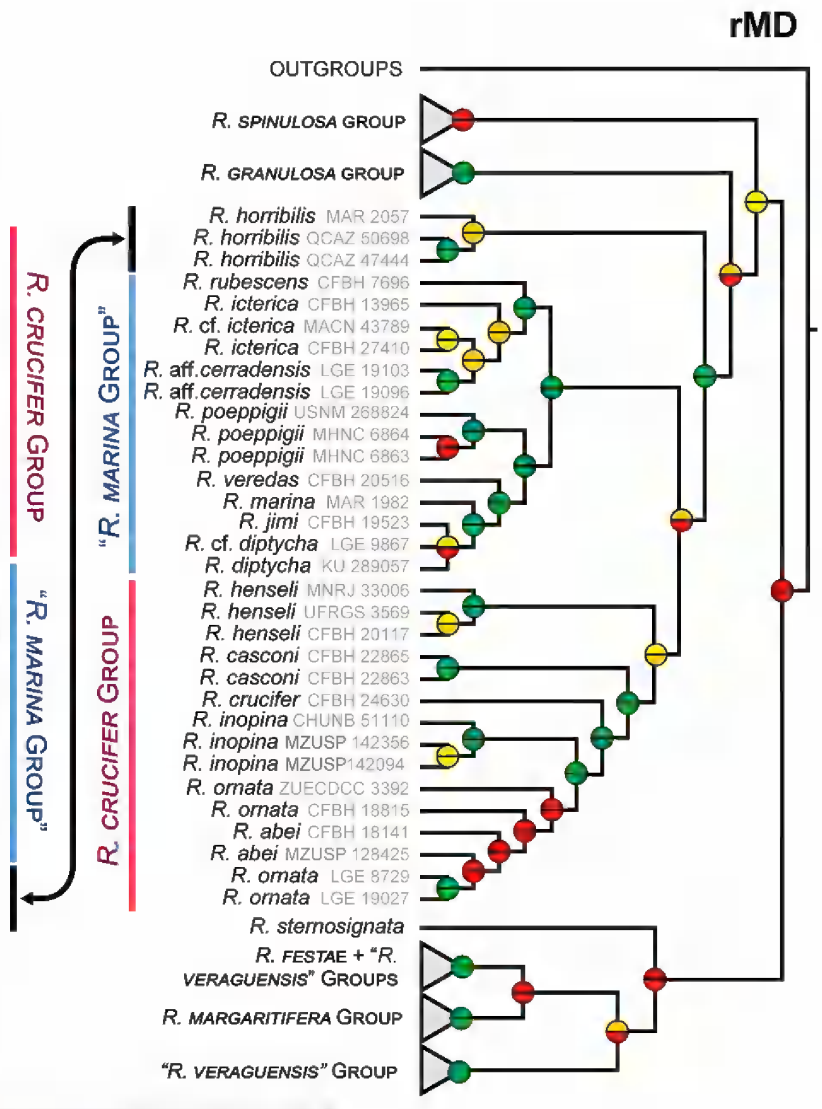

Jackknife support values (JGC and JAF)

O $\geq 98 \% \quad$ O $90 \%-97 \% \quad$ O $70 \%-89 \% \quad$ O $\leq 69 \%$

FIG. 9. Comparison between the strict consensuses resulting from the analyses of the restricted nuclear dataset (rND) and restricted mitochondrial dataset (rMD), showing the alternative positions of Rhinella horribilis in both analyses. Circles on nodes indicate parsimony jackknife frequencies (frequency differences value [above]/absolute [below]). Nodes lacking circles have $<25 \%$ frequency difference values or $<50 \%$ jackknife absolute frequencies.

(JGC $=63 \%, \mathrm{JAF}=73 \%)$. The clade composed of these three genera, in turn, is the sister taxon of a large and poorly supported clade (JGC $<25 \%$, JAF $<50 \%$ ) of African and Eurasian bufonids. An almost identical relationship was recovered in the ML analysis (see supplementary data 4.1). The sister-group relationship between Rhinella and Anaxyrus + Incilius is consistent with the results of most previous phylogenetic analyses (e.g., Pramuk, 2006: fig. 4; Pramuk et al., 2008; Pyron and Wiens, 2011; Pyron, 2014: suppl. information "amph_shl. tre"; Portik and Papenfuss, 2015; Jetz and Pyron,
2018: suppl. information "amph_shl_new.tre"). Alternatively, van Bocxlaer et al. (2010: fig. S1) recovered Rhinella as the sister taxon of a clade comprising all African and Eurasian bufonids.

Although the vast majority of species of Rhinella form an exclusive clade, it is polyphyletic because $R$. ceratophrys was recovered as the sister taxon of Rhaebo nasicus with strong support (JGC and JAF $=100 \%$ ). This relationship is not surprising, given that the morphological resemblance between both species was pointed out previously (e.g., Hoogmoed, 1977; Fenolio et al., 2012). 


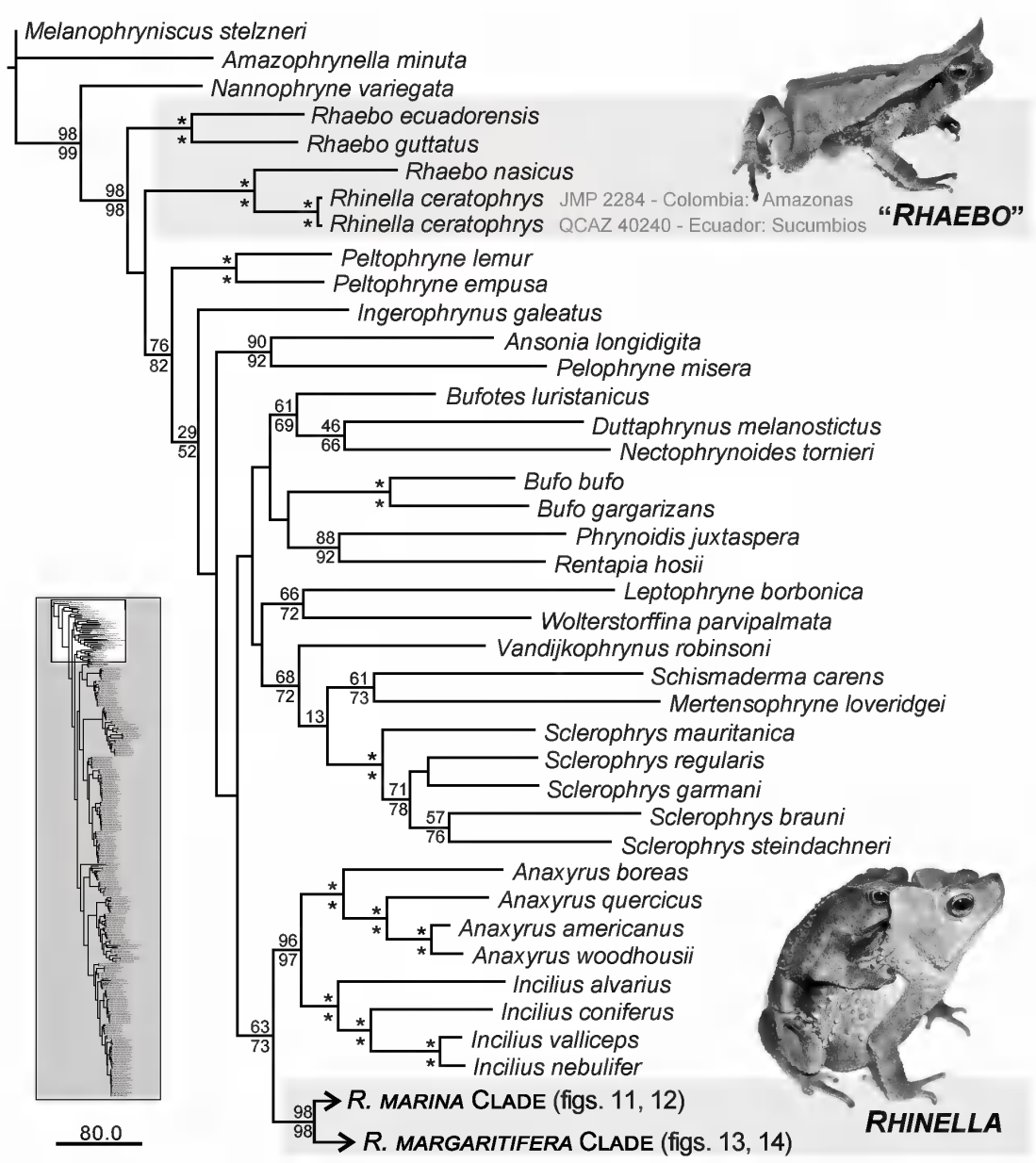

FIG. 10. Phylogenetic relationships of Rhinella and outgroups recovered in one of the most parsimonious trees from the total evidence analysis with TNT considering gaps as a fifth state (length 25,399 steps). Values around nodes are parsimony jackknife frequencies (frequency differences value [above]/absolute [below]). An asterisk $\left.{ }^{*}\right)$ indicates $100 \%$ jackknife support. Clades lacking references have $<25 \%$ frequency difference values or $<50 \%$ jackknife absolute frequencies. Lower left inset shows the entire cladogram with present view marked in white.

Although Rhaebo was paraphyletic in our TE analysis (fig. 10; but see results of the ML analysis in supplementary data 4.1), our taxon sampling was not designed to test its monophyly. Thus, we transfer Rhinella ceratophrys to Rhaebo as Rhaebo ceratophrys (Boulenger, 1882), new combination.

\section{RHINELLA AND ItS INTERNAL RELATIONSHIPS}

In the parsimony total evidence analysis, $R$ hinella was recovered as monophyletic (after trans- ferring $R$. ceratophrys to Rhaebo) and well supported (JGC and JAF $=98 \%$ ). The monophyly of Rhinella was previously recovered by several phylogenetic studies that used fewer taxa (e.g., Pauly et al., 2004: fig. 4; Pramuk, 2006; Pyron and Wiens, 2011; Pyron, 2014: suppl. information "amph_shl.tre"; Portik and Papenfuss, 2015; Jetz and Pyron, 2018: suppl. information "amph_ shl_new.tre"). In contrast to all previous studies, we found that Rhinella is composed of two major, well-supported clades (figs. 11-14; see below). 


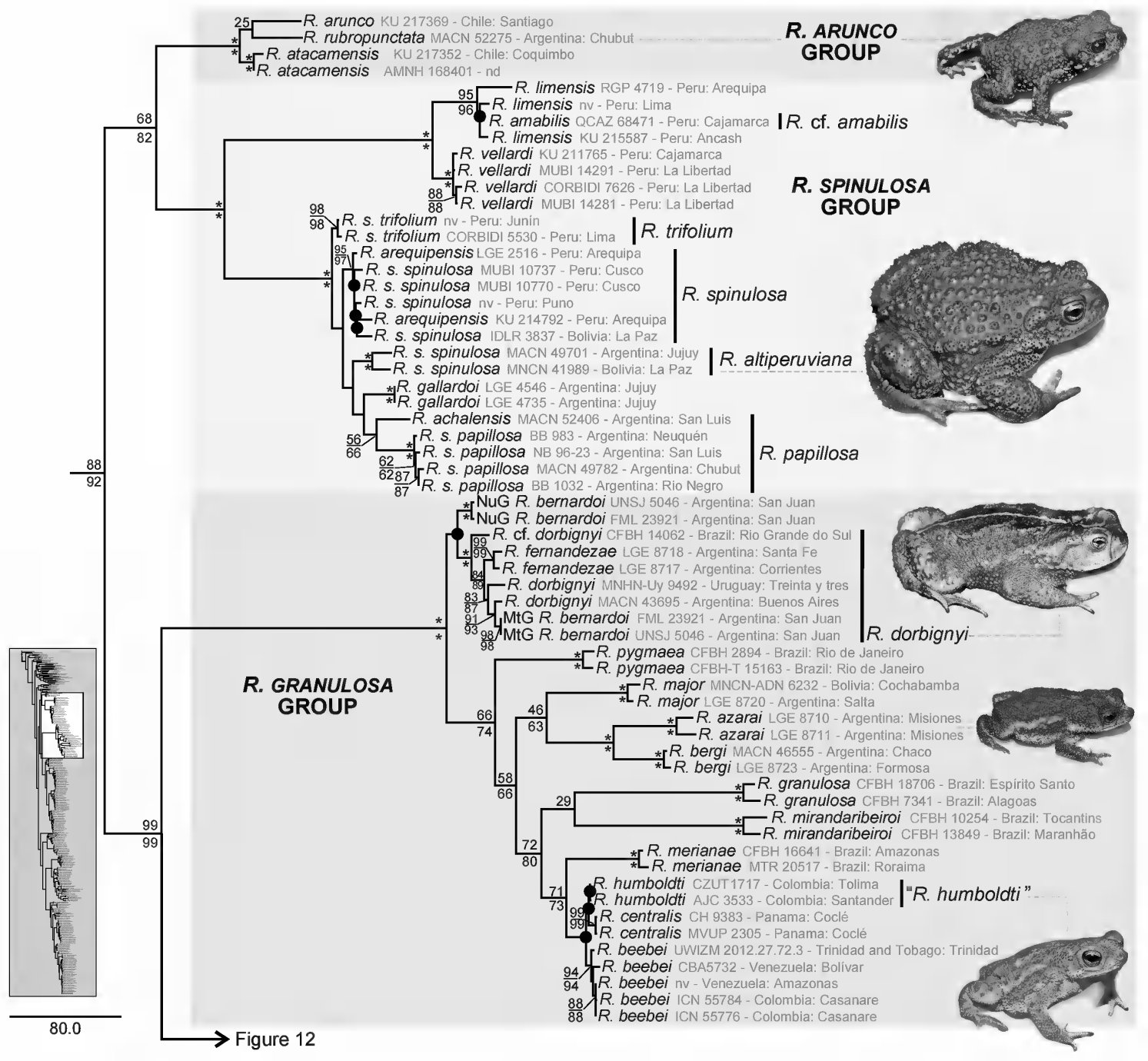

FIG. 11. Phylogenetic relationships of Rhinella recovered in one of the most parsimonious trees from the total evidence analysis with TNT considering gaps as a fifth state (length 25,399 steps). The clades and species groups shown are those recognized in this study. Part 1 of 4. The R. marina Clade (1): the R. arunco, R. spi$n u l o s a$, and $R$. granulosa Groups. Black circles indicate nodes that collapse in the strict consensus. Values around nodes are parsimony jackknife frequencies (frequency differences value [above]/absolute [below]). An asterisk $\left(^{*}\right)$ indicates $100 \%$ jackknife support. Clades lacking references have $<25 \%$ frequency difference values or $<50 \%$ jackknife absolute frequencies. Lower left inset shows the entire cladogram with present view marked in white. Abbreviations: $\mathbf{M t G}$, mitochondrial genome; $\mathbf{N u G}$, nuclear genome. 


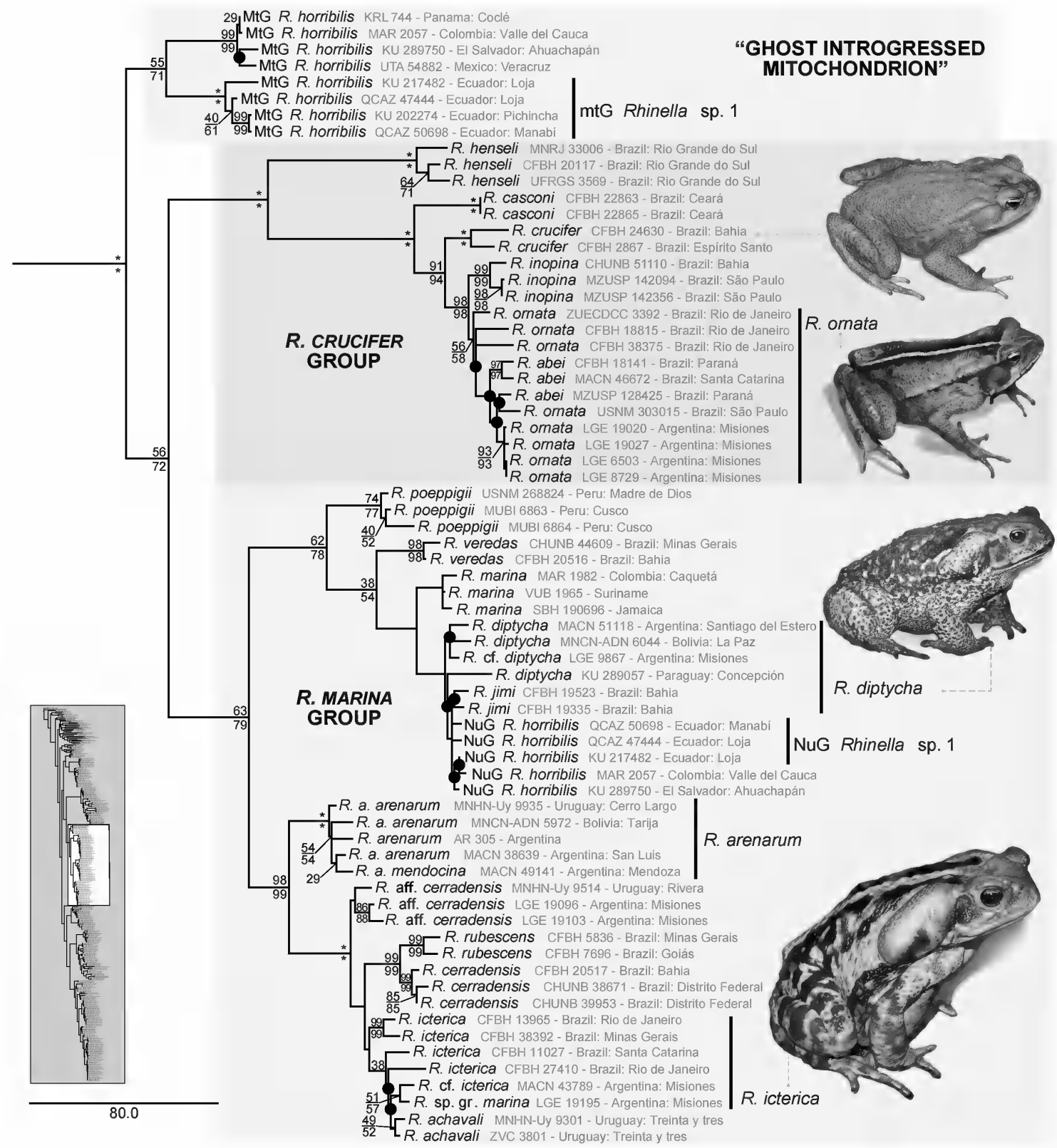

FIG. 12. Phylogenetic relationships of Rhinella recovered in one of the most parsimonious trees from the total evidence analysis with TNT considering gaps as a fifth state (length 25,399 steps). The clades and species groups shown are those recognized in this study. Part 2 of 4 . The R. marina Clade (2): the ghost introgressed mitochondrion and the R. crucifer and $R$. marina Groups. Black circles indicate nodes that collapse in the strict consensus. Values around nodes are parsimony jackknife frequencies (frequency differences value [above]/ absolute [below]). An asterisk $\left({ }^{*}\right)$ indicates $100 \%$ jackknife support. Clades lacking references have $<25 \%$ frequency difference values or $<50 \%$ jackknife absolute frequencies. Lower left inset shows the entire cladogram with present view marked in white. Abbreviations: MtG, mitochondrial genome; NuG, nuclear genome. 


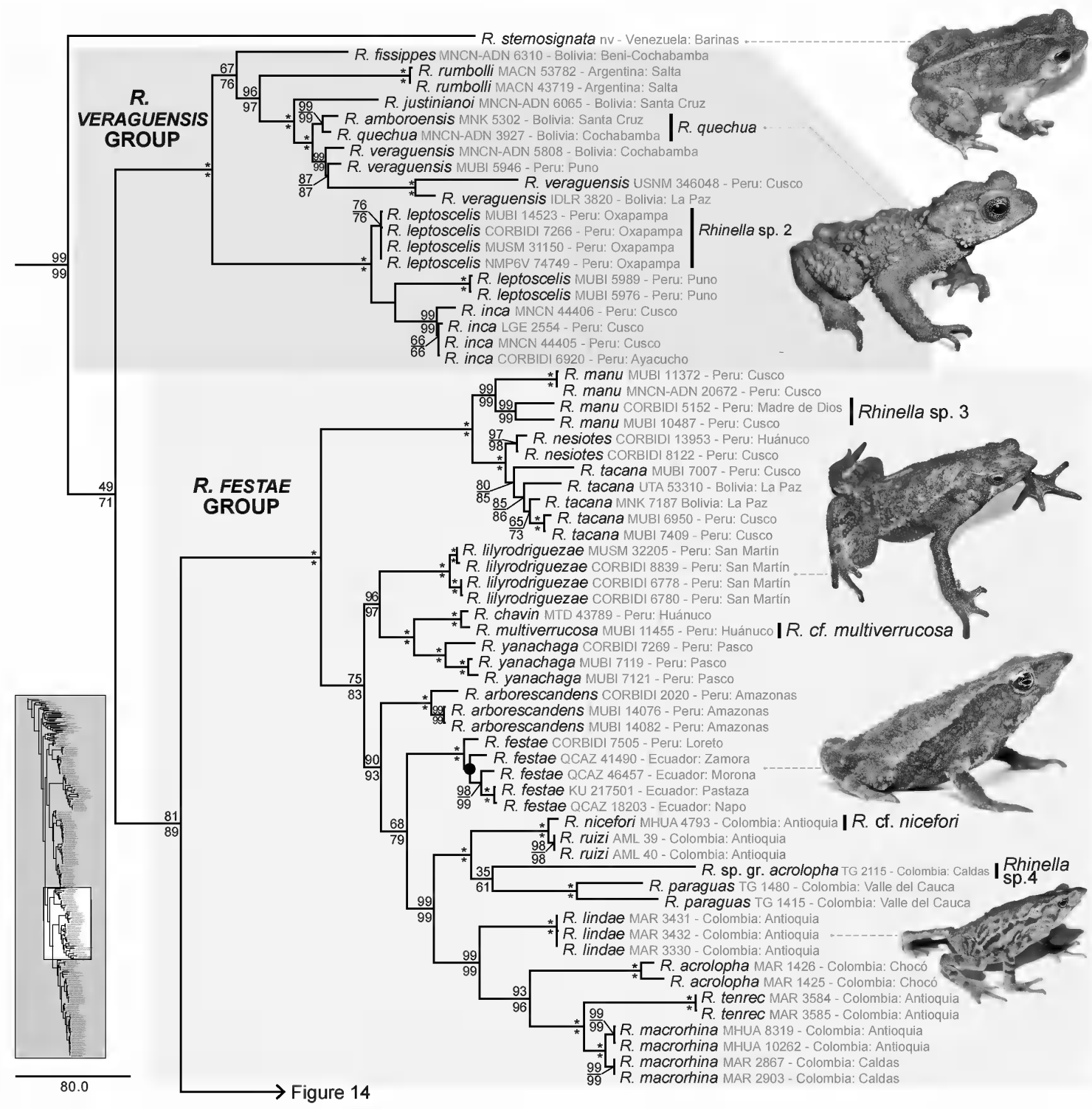

FIG. 13. Phylogenetic relationships of Rhinella recovered in one of the most parsimonious trees from the total evidence analysis with TNT considering gaps as a fifth state (length 25,399 steps). The clades and species groups shown are those recognized in this study. Part 3 of 4 . The R. margaritifera Clade (1): R. sternosignata and the $R$. veraguensis and $R$. festae Groups. Black circles indicate nodes that collapse in the strict consensus. Values around nodes are parsimony jackknife frequencies (frequency differences value [above]/absolute [below]). An asterisk $\left(^{*}\right)$ indicates $100 \%$ jackknife support. Clades lacking references have $<25 \%$ frequency difference values or $<50 \%$ jackknife absolute frequencies. Lower left inset shows the entire cladogram with present view marked in white. 


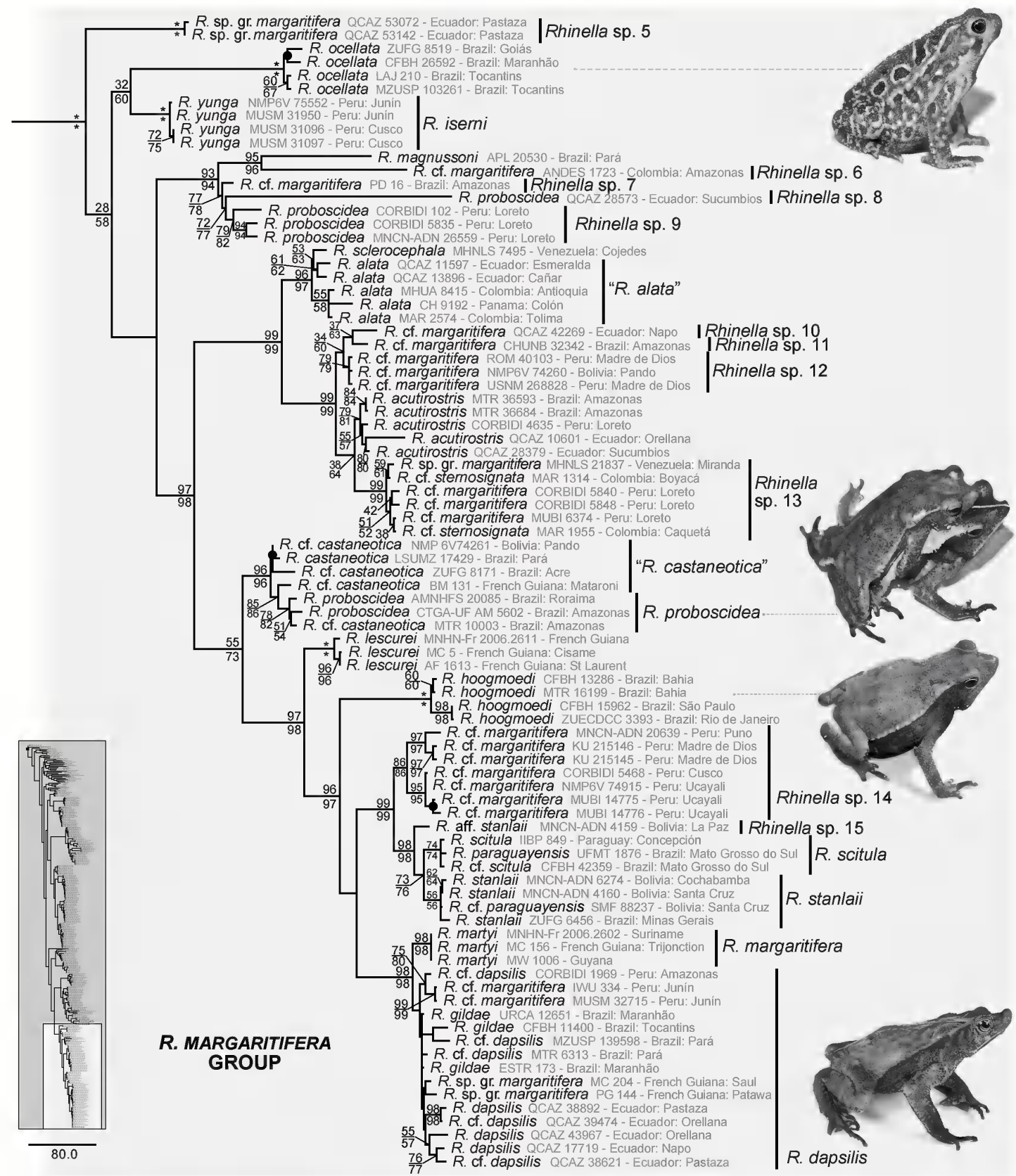

FIG. 14. Phylogenetic relationships of Rhinella recovered in one of the most parsimonious trees from the total evidence analysis with TNT considering gaps as a fifth state (length 25,399 steps). The clades and species groups shown are those recognized in this study. Part 4 of 4 . The $R$. margaritifera Clade (2): the $R$. margaritifera Group. Black circles indicate nodes that collapse in the strict consensus. Values around nodes are parsimony jackknife frequencies (frequency differences value [above]/ absolute [below]). An asterisk $\left(^{*}\right)$ indicates $100 \%$ jackknife support. Clades lacking references have $<25 \%$ frequency difference values or $<50 \%$ jackknife absolute frequencies. Lower left inset shows the entire cladogram with present view marked in white. 
Our results support the $R$. crucifer, $R$. granulosa, and $R$. marina Groups as monophyletic. Otherwise, the $R$. spinulosa Group is recovered paraphyletic due to the nested position of $R$. gallardo $i$ (a species unassigned to any group). The $R$. margaritifera Group is polyphyletic due to the position of the former $R$. ceratophrys nested in Rhaebo. The $R$. veraguensis Group is polyphyletic due to the position of several taxa (i.e., $R$. arborescandens, R. chavin, R. lilyrodriguezae, R. manu, $R$. multiverrucosa, $R$. nesiotes, $R$. tacana, and $R$. yanachaga) more closely related to the $R$. margaritifera Group, and with the monophyletic $R$. acrolopha Group nested within them. The ML analysis of the molecular + phenotypic datasets supported most of these results (supplementary data 4.2-4.5), and we only discuss the relevant differences between analyses. Below, we provide a revised account and comments for Rhinella and its main clades and species groups on the basis of these results.

\section{RHINELLA}

DiAgnosis: The long third pair of external gills (char. 79.2) optimizes as the only phenotypic synapomorphy of Rhinella in all the MPTs, which reverts to short third pair of external gills, the plesiomorphic bufonid condition, in the R. granulosa Group. An unequivocal diagnosis of this genus is obscured by the large phenotypic variation within Rhinella that overlaps with the diversity of many of the related bufonid genera. Nevertheless, this genus can be diagnosed from most of the related bufonids by the combination of the following phenotypic characters: (1) nasals and frontoparietal heavily ornamented with pits, striations, and rugosities (char. 9.2); (2) presence of a row of dorsolateral tubercles on skin (char. 57.1); and (3) nucleolar organizer regions (NORs) located on interstitial position of the short arms of the chromosome pair 7 (char. 89.4).

SISTER CLADE: The well-supported clade composed of Anaxyrus + Incilius (JGC $=96 \%$, $\mathrm{JAF}=97 \%)$.

Distribution: Mostly Neotropical, ranging from the southern United States to southern
South America. Rhinella marina is a highly invasive species introduced in many countries and islands outside its native distribution (e.g., Antilles, Australia, Hawaii, Philippines, Taiwan, etc.; see Frost, 2020; IUCN, 2020).

Comments: The exclusion of the former Bufo ceratophrys renders Rhinella monophyletic. The two large clades of Rhinella were not recovered in previous phylogenetic analyses (e.g., Pramuk, 2006: fig. 4; Chaparro et al., 2007; Pramuk et al., 2008; van Bocxlaer et al., 2010: suppl. information S1; Pyron and Wiens, 2011; Pyron, 2014: supp. information "amph_shl.tre"; Portik and Papenfuss, 2015; Pereyra et al., 2016a; Jetz and Pyron, 2018: suppl. information "amph_shl_new. tre"). Vera Candioti et al. (2016) proposed the long third pair of external gills as a putative synapomorphy of Rhinella in the context of a review of embryonic morphology of Bufonidae. Our TE analysis supports this character state as synapomorphy of the genus, although the embryonic morphology of many genera of Bufonidae and species of the $R$. margaritifera Clade (see below) is unknown. This synapomorphy of Rhinella reverts to the plesiomorphic state (short third pair of external gills) in the $R$. granulosa Group.

As a result of our TE analysis (also see ML result), we define two major clades, the Rhinella marina Clade and the $R$. margaritifera Clade, composed of eight species groups within $R h i$ nella. The $R$. marina Clade includes (1) the $R$. arunco Group (new species group); (2) the $R$. crucifer Group; (3) the R. granulosa Group; (4) the $R$. marina Group; and (5) the $R$. spinulosa Group as redefined here. The second clade, the $R$. margaritifera Clade, is composed of (1) $R$. sternosignata, a species unassigned to any group; (2) the R. festae Group as redefined here; (3) the R. margaritifera Group as redefined here; and (4) the $R$. veraguensis Group as redefined here. Below, we provide diagnoses, content, and comments on the distribution and systematics of each of the newly defined major clades and all species groups of Rhinella. The clades and species group are presented in the order described above and correspond to the sequence in which they 
appear in the TE tree (figs. 10-14) from base to tip and top to bottom.

\section{The Rhinella marina Clade (figs. 11, 12)}

Diagnosis: This clade is moderately supported $(\mathrm{JGC}=88 \%, \mathrm{JAF}=92 \%)$ and diagnosed by a phenotypic synapomorphy: larval otic capsule with poorly developed processus anterolateralis with a rounded aspect (char. 76.1), with one instance of homoplasy in Sclerophrys regularis.

SISTER ClADE: The Rhinella margaritifera Clade (figs. 13, 14).

Contents: The Rhinella marina Clade is composed of the R. crucifer, R. granulosa, and $R$. marina Groups, the $R$. spinulosa Group as redefined here, and the $R$. arunco Group, a new group defined here (see below). Moreover, we found a divergent mitochondrial lineage introgressed into $R$. horribilis (hereafter referred to as GIM [ghost introgressed mitochondrion], see below and discussion) that does not seem to belong to any recognized extant species of Rhinella and was recovered as sister clade of the $R$. marina + R. crucifer Groups (see fig. 12), although with poor support (see below).

Distribution: The species of this clade naturally occur in all main biogeographic regions of the Neotropics.

Comments: The Rhinella marina Clade is composed of two subclades. One is poorly supported $(\mathrm{JGC}=68 \%, \mathrm{JAF}=82 \%)$ and includes the $R$. arunco $+R$. spinulosa Groups (fig. 11). It is diagnosed by four phenotypic synapomorphies: (1) the supraorbital flange on frontoparietal does not extend laterally beyond the lateral margin of the sphenethmoid (char. 17.0, with instances of homoplasy in R. quechua and some outgroups); (2) the m. extensor digitorum on the metacarpophalangeal joint of digiti III (char. 44.1, with instances of homoplasy in Anaxyrus woodhousii [polymorphic], Rhinella hoogmoedi, R. jimi, and R. rumbolli); (3) parotoid gland round to ovoid, mostly symmetrical (char. 56.2, with instances of homoplasy in $R$. bergi and several species of the $R$. margaritifera Clade); and (4) vocal sac absent in adult males (char. 58.0, with instances of homoplasy within Rhinella and outgroups). The other subclade is well supported (JGC and JAF = 99\%) and includes the $R$. crucifer, $R$. granulosa, and R. marina Groups, and the GIM (figs. 11, 12). Three phenotypic synapomorphies are recovered for this subclade: (1) occurrence of a well-developed supraorbital crest (char. 1.2, with instances of homoplasy in several bufonids); (2) occipital artery pathway completely covered with bone (char. 10.2, with instances of homoplasy in bufonids); and (3) general pattern of coloration of caudal musculature of larvae uniformly dark except an unpigmented longitudinal stripe along the inferior edge (char. 69.1, with instances of homoplasy in $R$. quechua, $R$. veraguensis, and some outgroups).

Previous phylogenetic studies including less complete sampling of Rhinella (Pramuk, 2006; Pramuk et al., 2008; van Bocxlaer et al., 2010; Pyron and Wiens, 2011; Pyron, 2014; Portik and Papenfuss, 2015; Pereyra et al., 2016a; Jetz and Pyron, 2018) never found a sister relation between the clade composed of the R. arunco + $R$. spinulosa Groups and the clade composed of the $R$. granulosa $+(R$. crucifer $+R$. marina $)$ Groups. Instead, these studies found the $R$. arunco and $R$. spinulosa Groups as: (1) the sister clade of the species of the $R$. margaritifera Clade as defined here (Pramuk, 2006), (2) as sister clade of the remaining species of Rhinella (Pramuk et al., 2008; Pereyra et al., 2016a), or (3) as successive sister clades of the remaining species of Rhinella (van Bocxlaer et al., 2010; Pyron and Wiens, 2011; Pyron, 2014; Portik and Papenfuss, 2015; Jetz and Pyron, 2018). In contrast to all these analyses, our ML analysis recovers the $R$. arunco Group as the sister clade of the remainder of the $R$. marina Clade, whereas the $R$. spinulosa Group is the sister taxon of the clade composed of $R$. granulosa + ( $R$. crucifer $+R$. marina) Groups. This last clade has always been recovered as monophyletic in previous phylogenetic analyses (Pramuk, 2006; van Bocxlaer et al., 2010; Pyron and Wiens, 2011; Pyron, 2014; Portik and Papenfuss, 2015; Pereyra et al., 2016a; Jetz and Pyron, 2018). 


\section{The Rhinella arunco Group}

Diagnosis: Two phenotypic synapomorphies diagnose this strongly supported (JGC and JAF $=100 \%)$ species group: (1) jaw articulation opposite to the fenestra ovalis (char. 16.1, with instances of homoplasy in some species of the Rhinella granulosa Group, the R. margaritifera Clade, and in Nannophryne variegata); and (2) anterior edge of sacral diapophyses perpendicular to the midline axis of the vertebral column (char. 30.1, with instances of homoplasy in $R$. crucifer, $R$. quechua, $R$. rubescens, $R$. spinulosa, and $R$. vellardi). The presence of an insertion of the $\mathrm{m}$. extensor digitorum longus on metatarsophalangeal joint of digit $\mathrm{V}$ (char. 40.1 ) and the presence of an insertion of the $\mathrm{m}$. extensor digitorum on metacarpophalangeal joint of digiti V (char. 45.1) could represent two additional synapomorphies of this group or an internal clade. Moreover, species of the $R$. arunco Group can be distinguished from members of the other species groups of Rhinella by the following combination of character states: (1) preorbital crest weak (char. 0.1), (2) occipital artery pathway uncovered with bone (char. 10.0), (3) frontoparietal that does not extend laterally beyond the lateral margin of the sphenethmoid (char. 17.0), (4) medial ramus of the pterygoid fused with the anterolateral margin of the parasphenoid (char. 21.1), (5) m. extensor digitorum longus with an insertion on the metatarsophalangeal joint of the digit IV (char. 39.1), (6) m. extensor digitorum with an insertion on the metacarpophalangeal joint of digiti III (char. 44.1), (7) inguinal fat bodies present (char. 51.1), (8) row of dorsolateral tubercles absent (char. 57.0), (9) vocal sac absent in adult males (char. 58.0), and (10) eggs biserially disposed in strings (char. 87.1).

Sister Clade: The Rhinella spinulosa Group. Contents (3 species): Rhinella arunco (Molina, 1782), R. atacamensis (Cei, 1962), and R. rubropunctata (Guichenot, 1848).

Distribution: Species of the Rhinella arunco Group are distributed in Argentina and Chile: Rhinella arunco and R. atacamensis in the Atac- ama Desert region, $R$. rubropunctata in the Austral Temperate Forest region (Cei, 1962, 1980; Correa et al., 2013). See map 1 (available at https://doi.org/10.5531/sd.sp.46) for type localities and sampled localities.

Comments: Pramuk (2006) found the Rhinella spinulosa Group (sensu Duellman and Schulte, 1992) as nonmonophyletic and excluded the species now placed in Nannophryne (i.e., $N$. apolobambica, $N$. cophotis, $N$. corynetes, and $N$. variegata; see Frost et al., 2006; Frost, 2020). The remaining species constituted a well-supported clade in her combined (morphological and molecular) analysis, being the sister taxon to all the remaining species of Rhinella. A subsequent molecular phylogeny (Pramuk et al., 2008) considering a similar taxon sampling and mitochondrial genes, but several different nuclear genes with respect to Pramuk (2006; cxcr4 and rag1-a vs pomc and rag1-a), recovered this redelimited group as monophyletic with poor support. Previous and subsequent analyses with slightly increased taxon and gene sampling, however, found this group as paraphyletic with respect to all remaining species groups of Rhinella (Frost et al., 2006; van Bocxlaer et al., 2010; Pyron and Wiens, 2011; Pyron, 2014; Portik and Papenfuss, 2015; Jetz and Pyron, 2018), or as the (poorly supported) sister taxon of all other species of Rhinella (Pereyra et al., 2016a). In our TE analysis, the former R. spinulosa Group (including $R$. gallardoi, see below) was recovered as monophyletic but poorly supported (JGC $=68 \%, \mathrm{JAF}=$ $82 \%)$. Moreover, the individual monophyly of its sister subclades is strongly supported (both with JGC and JAF $=100 \%$ ) and can be diagnosed by phenotypic synapomorphies (see Diagnosis of both groups). Our ML analysis found the former R. spinulosa Group paraphyletic with respect to the remaining species groups of the $R$. marina Clade (supplementary data 4.2). Based on these observations, we restrict the R. spinulosa Group to the strongly supported clade containing most species of the former $R$. spinulosa Group (and including $R$. gallardoi), and exclude the extraAndean species $R$. arunco, $R$. atacamensis, and $R$. 
rubropunctata that constitute another well-supported clade, herein recognized as the $R$. arunco Group. The southernmost distributed species $R$. arunco and $R$. rubropunctata are recovered as sister taxa, although with poor support (JGC $=25$, $\mathrm{JAF}<50 \%)$. The three species of this group show a high genetic differentiation in comparison to other species groups of the $R$. marina Clade (see tables 3-6). Natural hybridization between $R$. arunco and $R$. atacamensis was reported by Correa et al. (2012, 2013), but they did not find mitochondrial and nuclear introgression outside a narrow hybrid zone.

\section{The RHINELla SPINUlosa Group}

Diagnosis: The following character states optimize as phenotypic synapomorphies of this strongly supported group (JGC and JAF = 100\%) in our TE analysis: (1) pretympanic crest absent or indistinguible (char. 2.0, with instances of homoplasy in Rhinella arunco, R. castaneotica, $R$. festae, and some outgroups); (2) nasal and frontoparietal bones articulating only laterally (char. 8.1, homoplastic in R. quechua, R. rubropunctata, $R$. veraguensis, $R$. yanachaga, Rhinella sp. 14, and some outgroups); (3) lightly exostosed dermal roofing bones (char. 9.1, homoplastic in the $R$. festae Group, in several species of the $R$. marina Group, and outgroups); and (4) slightly enlarged otic ramus of squamosal, overlapping with the dorsal surface of the crista parotica (char. 11.1). In addition, species of the $R$. spinulosa Group can be distinguished from members of the other species groups of Rhinella by the following combination of character states: (1) occipital artery pathway not covered by bone (char. 10.0), (2) frontoparietal that does not extend laterally beyond the lateral margin of the sphenethmoid (char. 17.0), (3) medial ramus of the pterygoid fused with the anterolateral margin of the parasphenoid (char. 21.1), (4) m. extensor digitorum longus with an insertion on metatarsophalangeal joint of digiti IV (char. 39.1), (5) m. extensor digitorum with an insertion on the metacarpophalangeal joint of digiti III (char. 44.1), (6) inguinal fat bodies present (char. 51.1), (7) mul- tiserial configuration of eggs in the jelly string (char. 87.2), (8) tarsal fold present (char. 65.1), and (9) adhesive gland divided after fusion of the operculum in embryo (char. 82.2).

Sister Clade: The Rhinella arunco Group.

CONTENTS (9 sPeCIEs): Rhinella achalensis (Cei, 1972b), R. altiperuviana (Gallardo, 1961) new status, $R$. amabilis (Pramuk and Kadivar, 2003), R. gallardoi (Carrizo, 1992), R. limensis (Werner, 1901), R. papillosa (Philippi, 1902), new status, $R$. spinulosa (Wiegmann, 1834) [including $R$. arequipensis (Vellard, 1959), new synonymy, see below], $R$. trifolium (Tschudi, 1845) new status, and $R$. vellardi (Leviton and Duellman, 1978).

Distribution: This species group is mostly distributed in arid regions along the Andes of Argentina, Bolivia, Ecuador, Chile, and Peru, except Rhinella gallardoi that inhabits the humid subandean forest of Argentina (Vellard, 1959; Córdova, 1999; Pramuk and Kadivar, 2003; Lavilla and Cei, 2001). Rhinella achalensis and $R$. limensis are the only species of this group with an extra-Andean distribution in the Sierras Pampeanas Centrales in the Pampas region of Argentina and Atacama Desert of Peru respectively (Vellard, 1959; Cei, 1972b). See map 2 (available at https://doi.org/10.5531/sd.sp.46) for type localities and sampled localities.

Comments: The Rhinella spinulosa Group as redelimited here is composed of some taxa with controversial taxonomies that are discussed in the context of our results. The widespread, polytypic, and poorly defined species $R$. spinulosa is recovered as nonmonophyletic, with $R$. achalensis, $R$. gallardoi, and $R$. arequipensis nested within it (fig. 11). Based on our results and considering that "Peru" is the type locality of $R$. spinulosa (and putatively confined to southern Peru, see Vellard, 1959), we restrict the species $R$. spinulosa s.s. to the well-supported lineage (JGC = $95 \%$, JAF $=97 \%$ ), composed of the populations from southern Peru and northern Bolivia. The lineage containing these populations of $R$. spinulos $a$ also includes the sampled specimen of $R$. arequipensis from Departamento Arequipa, Peru. Rhinella arequipensis was originally described as 
a subspecies of $R$. spinulosa based only on differences in coloration and density of granular formations in the dorsal tegument (Vellard, 1959). Morrison (1992, 1994), Córdova (1999), and Aguilar and Gamarra (2004) did not find morphological, osteological, karyological, or larval differences that could discriminate between $R$. spinulosa and $R$. arequipensis. According to these observations and our results, we consider Bufo spinulosus arequipensis Vellard, 1959, a junior synonym of Rhinella spinulosa (Wiegmann, 1834). Thus, the species $R$. spinulosa is restricted to the populations distributed mainly along the Andean Puna of Peru and adjacent Bolivia.

Populations of Rhinella spinulosa that had been considered as $R$. s. trifolium were recovered as a distinct and strongly supported lineage (JGC and $\mathrm{JAF}=98 \%$ ) sister to a poorly supported clade (JGC $<25 \%$, JAF <50\%) containing $R$. spinulosa s.s. and several other species of the group (see below). There are several morphological differences between $R$. s. trifolium and R. spinulosa s.s. Vellard (1959) pointed out the disposition of the dorsal glands (longitudinal rows in R. s. trifolium and a uniform distribution in R. s. spinulosa) and the occurrence of a middorsal vertebral line in R. s. trifolium, as the main distinguishing characters. Morrison (1992, 1994), Sinsch (1986), Haas (2002), and Pramuk and Kadivar (2003) considered $R$. spinulosa s.s. and R. s. trifolium (and also R. s. flavolineata) as variations of a single species (see below), although all but Haas failed to provide detailed justification. The morphological comparisons were some superficial and a detailed reevaluation of the specimens and comparisons with topotypes is needed. Córdova (1999) and Aguilar and Gamarra (2004) did not find karyological or larval differences between $R$. s. spinulosa and $R$. s. trifolium; however, these character systems are conserved in related species of Rhinella (see Tolledo and Toledo, 2010; Kolenc et al., 2013; Blotto et al., 2014). The UPDs between the specimens of $R$. s. trifolium and $R$. spinulosa s.s. are relatively high for this species group (1.11\%-1.30\%, see table 4$)$. Consequently, the differences in adult morphology proposed by
Vellard (1959) and their genetic divergence support the recognition of Rhinella trifolium (Tschudi, 1845) as a distinct species.

Some populations currently assigned to Rhinella spinulosa s.l. from Jujuy (Argentina) and La $\mathrm{Paz}$ (Bolivia) were recovered as another distinct and strongly supported lineage (JGC and JAF = $100 \%$; see fig. 4) with a low UPD between them $(0.18 \%)$. In the intermediate area of Puna between these localities $(\sim 800 \mathrm{~km})$ lays the type locality of $R$. s. altiperuviana (Challapata, Oruro, Bolivia). Gallardo (1961) described this subspecies from two adult females; the characters used to differentiate it from $R$. spinulosa s.l. (i.e., tubercles structure, head shape, tarsal fringe development) show considerable variation, at least, in the studied female specimens from northwestern Argentina. Thus, we tentatively assign these populations to $R$. s. altiperuviana. In addition to the phylogenetic position, these specimens differ in UPDs (see table 4) and adult and larval external morphology (B.L.B., D.B., M.O.P., personal obs.) from other species of the group. For these reasons, these populations should be considered as a distinctive species, $R$. altiperuviana (Gallardo, 1961) from the Andean Puna of Argentina and Bolivia. A detailed taxonomic revision is beyond the scope of this work but will be discussed in a subsequent contribution (B.L.B. and M.O.P., in prep.).

Populations of Rhinella spinulosa that had been considered as $R$. s. papillosa are recovered as a strongly supported lineage (JGC and JAF = $100 \%$ ), sister taxon of $R$. achalensis. Both taxa differ in UPDs (1.10 to $1.47 \%$, see table 4 ), and are morphologically differentiable from $R$. spinulosa s.s. (B.L.B. and M.O.P., in prep.). Thus, we consider R. papillosa (Philippi, 1902), a valid species from the austral Andes of Argentina and Chile.

Rhinella gallardoi is deeply nested within the $R$. spinulosa Group. In the original description, Carrizo (1992) highlighted the "broad skull" of this species over the general morphological similarity with the species of the $R$. spinulosa Group and assigned it to the "Bufo veraguensis-typho- 


\section{TABLE 3}

\section{Percentage of uncorrected p-distances between $16 \mathrm{~S}$ sequences among species of the Rhinella arunco Group} Values reported are mean (range).

\begin{tabular}{llccc}
\hline \hline & \multicolumn{1}{c}{1} & 2 & 3 \\
\hline 1 & $\begin{array}{l}\text { R. arunco } \\
(\mathrm{N}=1)\end{array}$ & - & & \\
\hline 2 & $\begin{array}{l}\text { R. rubropunctata } \\
(\mathrm{N}=1)\end{array}$ & 2.87 & - & \\
\hline 3 & $\begin{array}{l}\text { R. atacamensis } \\
(\mathrm{N}=2)\end{array}$ & $\begin{array}{c}2.80 \\
(2.67-2.93)\end{array}$ & $\begin{array}{c}2.41 \\
(2.28-2.55)\end{array}$ & 0.42 \\
\hline
\end{tabular}

nius" complex. Moreover, $R$. gallardoi is the only species of the $R$. spinulosa Group inhabiting exclusively the Yungas of the Andes in northwestern Argentina.

The specimens of Rhinella amabilis, $R$. limensis, and $R$. vellardi are recovered as a strongly supported clade (JCG and JAF and $=100 \%$ ), which is the sister taxon of the remaining species of the $R$. spinulosa Group as redefined here. Within this clade, the specimen of $R$. amabilis collapses into a basal polytomy with the specimens of $R$. limensis (the UPDs within the clade composed of these specimens are low $[0.19 \%-0.44 \%])$. Rhinella amabilis was differentiated from $R$. limensis by a few characters (development of cranial crests, presence of vocal slits, and shape of the parotoid gland). Rhinella amabilis is the only species of the R. spinulosa Group distributed north of the Huancabamba depression (Loja, Ecuador), and we could not obtain tissues from this area. The only specimen sampled of this species comes from a locality in the Huancabamba depression region but does not fully correspond with the morphological description of the species. An extensive revision of both species, including topotypical material and comparison with the holotypes is necessary to test the validity of $R$. amabilis.

The currently recognized subspecies Rhinella spinulosa flavolineata was not included in our analyses. This subspecies differs from $R$. trifolium only in the conspicuity and time of emergence of the vertebral line. Haas (2002) studied the development of specimens he assigned to the subspecies R. s. spinulosa, R. s. trifolium, and $R$. s. flavolineata from the same locality (Mantaro valley, between Concepcion and Huancayo, Junin department, Peru). This author reported that juveniles with variable development (or even absence) of this vertebral line could be obtained from a single clutch, hence, this character seems not to be relevant in differentiating these taxa. The occurrence of $R$. spinulosa s.s. in that locality is debatable (see Vellard, 1959, for comments on the distributions of these taxa) and it is possible that Haas (2002) assigned specimens of $R$. trifolium with poorly defined vertebral line to R. spinulosa s.s. (see Haas, 2002: fig. $1)$. In any case, the results of that study demonstrate that the tempo and level of development of the vertebral line are highly variable. Considering that the different morphs found by this author correspond to intraspecific variation within R. trifolium, we consider Bufo spinulosus flavolineatus Vellard, 1959, a junior synonym of Rhinella trifolium (Tschudi, 1845). Rhinella trifolium is considered to inhabit the Central Andean Wet Puna (Vellard, 1959), but additional studies are necessary to determine the precise limits of its geographic distribution and variation with respect to $R$. spinulosa s.s.

\section{The RHINella granulosa Group}

Diagnosis: This species group is recovered as monophyletic with strong support (JGC and JAF $=100 \%$ ) as in previous analyses (Pramuk, 2006; Pereyra et al., 2016a). Four phenotypic 


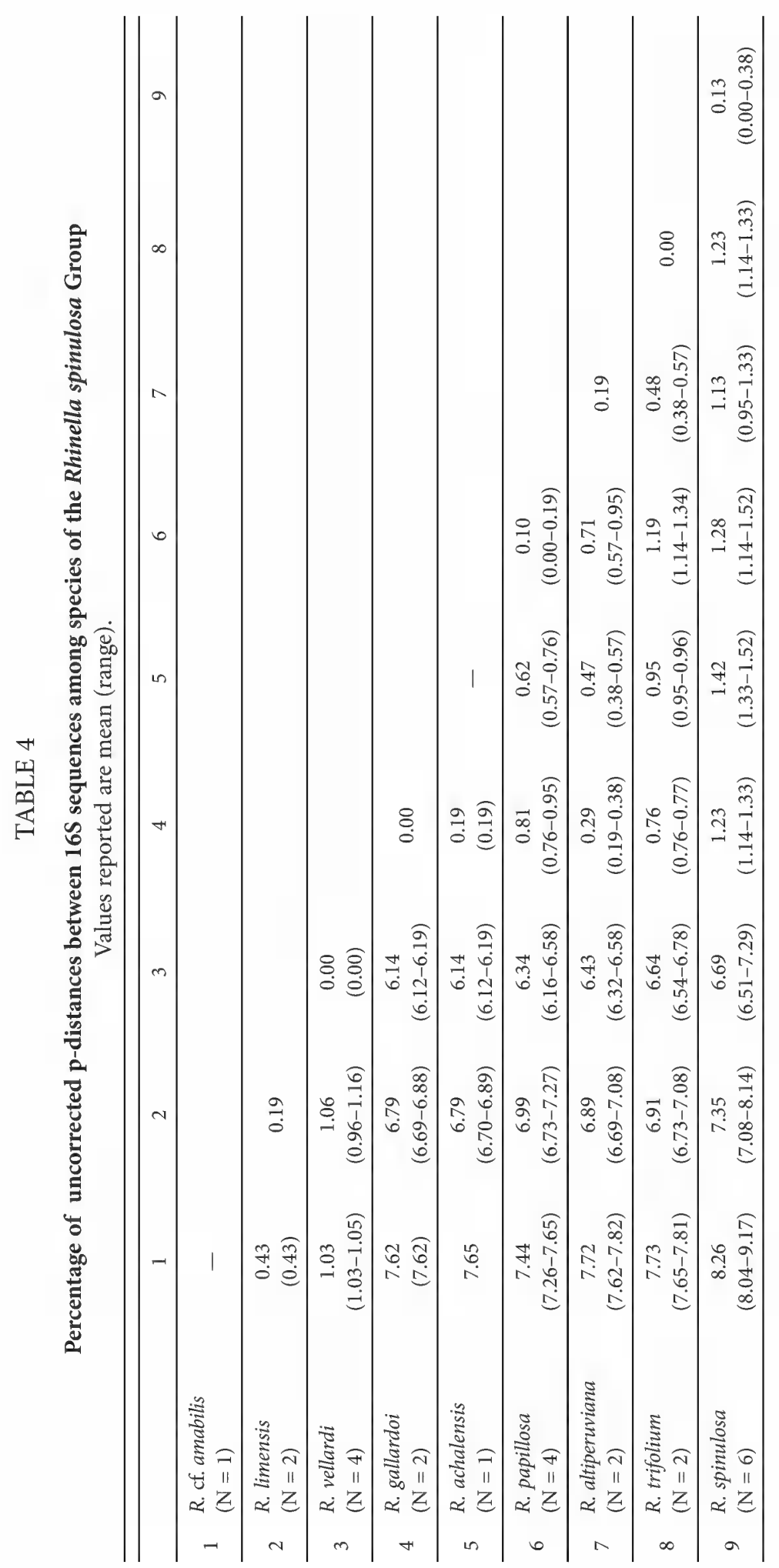


synapomorphies are recovered for this group: (1) anterior end of the septomaxilla developed (previously considered to be the prenasal bones; see discussion of this character in List and Description of Characters) (char. 14.1); (2) sacral diapophyses with the maximum width greater than its maximum length (char. 29.1), with several instances of homoplasy in Rhinella and outgroups; (3) submarginal papillae in the larval oral disc absent (char. 71.0), with instances of homoplasy in several bufonids; and (4) two posterior labial tooth rows in the larval oral disc (char. 72.0), that revert in an internal clade of this group. Moreover, nine additional characters might represent synapomorphies of this group or an internal clade depending on their occurrence in $R$. bernardoi and $R$. dorbignyi, where they are still unknown: (1) anteriorly oriented alary process of the premaxilla (char. 13.2), which also optimizes as a synapomorphy of the R. margaritifera Clade and is homoplastic in Incilius coniferus, Schismaderma carens, and some species of the R. marina Clade; (2) articulation of the zygomatic ramus of the squamosal with the maxilla (char. 15.1), homoplastic in Peltophryne lemur and R. sternosignata; (3) articulation of the jaw anterior to the fenestra ovalis (char. 16.2), homoplastic in Melanophryniscus gr. stelzneri and Peltophryne lemur; (4) bony sphenethmoid reaching the level of the premaxillae anteriorly (char. 18.2); (5) posterior lobe in the anterolateral process of hyoid absent (char. 25.0), homoplastic in Rhaebo ceratophrys, Rhinella acrolopha, and in the $R$. margaritifera Group; (6) vocal sac projected anteriorly when fully inflated (char. 60.1), homoplastic in some species of Anaxyrus; (7) short third pair of gills in the embryos (char. 79.1), homoplastic in Melanophryniscus gr. stelzneri and Schismaderma carens; (8) short dorsal line of hatching glands in the embryos (char. 80.0), with an instance of homoplasy in R. marina; and (9) type-A adhesive glands in the embryos (char. 81.0).

The species of the Rhinella granulosa Group can be distinguished from members of the other groups of Rhinella by the following combination of character states: (1) preorbital crest well developed (char. 0.2 ), (2) supraorbital crest well developed (char. 1.2 ), (3) pretympanic crest well developed (char. 2.2), (4) nasal and frontoparietal articulate along the entire margin (char. 8.3), (5) occipital artery pathway completely covered with bone (char. 10.2), (6) medial ramus of the pterygoid fused and extending medially along approximately half the length of the parasphenoid alae (char. 21.2), (7) anterior edge of sacral diapophyses perpendicular to the midline axis of the vertebral column (char. 30.1), (8) inguinal fat bodies present (char. 51.1), (9) tarsal fold absent (char. 65.0), (10) caudal musculature of larvae uniformly dark except an unpigmented longitudinal stripe along the inferior edge (char. 70.1), (11) occurrence of irregular transverse whitish stripes in the caudal musculature of larvae (char. 70.1), (12) short third gill pair in the embryo (char. 79.1), and (13) adhesive gland divides immediately before the gills reach their maximum development (char. 82.1).

Sister ClADE: The clade composed of the GIM (see below) and the Rhinella crucifer and $R$. marina Groups.

Contents (13 species): Rhinella azarai (Gallardo, 1965); R. beebei (Gallardo, 1965); R. bergi (Céspedez, 2000); $R$. bernardoi Sanabria et al., 2010; R. centralis Narvaes and Rodrigues, 2009; $R$. dorbignyi (Duméril and Bibron, 1841) [including $R$. fernandezae (Gallardo, 1957) new synonymy, see below]; R. granulosa (Spix, 1824); $R$. humboldti (Gallardo, 1965); R. major (Müller and Hellmich, 1936); R. merianae (Gallardo, 1965); R. mirandaribeiroi (Gallardo, 1965); $R$. nattereri (Bokermann, 1967); and R. pygmaea (Myers and Carvalho, 1952).

Distribution: Species of this group are widely distributed in open areas of Amazonia, Atlantic Forest, Caatinga, Cerrado, Chaco/ Pantanal, Chocó, and Pampas regions and in Panama (Narvaes and Rodrigues, 2009; Sanabria et al., 2010; Pereyra et al., 2016a; Murphy et al., 2017). See map 3 (available at https://doi.org/10.5531/sd.sp.46) for type localities and sampled localities. 
COMMENTs: In a previous molecular phylogenetic analysis of the Rhinella granulosa Group, Pereyra et al. (2016a) recognized 12 putative phenotypic synapomorphies for the group (three of these were first proposed by Pramuk, 2006, and one by Blotto et al., 2014). Eight of these character states were included as part of homology hypotheses (characters) in our TE analysis and only three were corroborated as synapomorphies of the R. granulosa Group in all the MPTs (all the remaining were recovered as synapomorphies in some MPTs). The remaining four character states (i.e., the presence of an expanded flag-shaped dorsal crest of the ilium in lateral view; nasal bone articulates with the dorsal margin of the pars facialis of the maxilla from the preorbital process to the posterior margin of the narial opening; occipital condyles widely separated; and ability to build and inhabit holes in the ground) were not included due to the lack of detailed descriptions or preparations for many species of Rhinella. However, these character states are unique of the $R$. granulosa Group among the most closely related groups and are consequently considered putative synapomorphies of this group.

Taxonomic, genetic, and biological aspects of the Rhinella granulosa Group were addressed in detail by Pereyra et al. (2016a), but some differences need to be stressed. First, we found variations in the inferred relationships among the earlier diverging clades/species of this group. Our TE analysis recovered a basal polytomy that comprises: (1) R. bernardoi, (2) R. dorbignyi (including $R$. fernandezae, see below), and (3) a poorly supported clade $(\mathrm{JGC}=66 \%$, JAF $=74 \%$ ) composed of the remaining species of the group. Pereyra et al. (2016a) found $R$. major to be the sister species of a poorly supported clade (JAF $<50$, no JGC value reported) comprising all the remaining species of the group. Although in both analyses the interspecific relationships are poorly supported in general, we presume that these differences are due to the inclusion of phenotypic characters, the inclusion of sequences of $R$. humboldti, the denser outgroup sampling in this study, and the inclusion of a contaminated fragment of cytochrome $b$ (KP684992; contaminated with $R$. icterica) in the dataset of Pereyra et al. (2016a).

Pereyra et al. (2016a) retained Rhinella dorbig$n y i$ and $R$. fernandezae as different species, although they noted the absence of reciprocal monophyly between both taxa and the very low genetic distances among the sampled specimens. Although we did not add additional specimens or sequences to our analyses (but a set of phenotypic characters was added in the TE analysis) and we recovered the same topology as Pereyra et al. (2016a) for the clade containing both taxa, we consider Bufo granulosus fernandezae Gallardo, 1957, a junior synonym of Rhinella dorbignyi (Duméril and Bibron, 1841). This decision is consistent with the criteria followed to synonymize other taxa of Rhinella (i.e., absence of reciprocal monophyly, absence of genetic differentiation, and absence of conspicuous differential morphological characters). Different populations of $R$. dorbignyi s.s. vary only in the level of development of the cranial crest, but not in other phenotypic or molecular characters. We hypothesize that local environmental factors through the area of distribution (i.e., Espinal, Humid Chaco, Humid Pampa, and Uruguayan Savanna) could affect the levels of ossification in the skull, resulting in differential development of cranial crests. The genetic and environmental causes of hyperossification are still not well understood in anurans (Paluh et al., 2020; Blotto et al., 2021). The differential patterns of bone deposition on the skull of $R$. dorbignyi are drastic and generate large morphological differences, making this species an excellent candidate to explore the role and impact of environmental factors on hyperossification.

We recovered Rhinella humboldti as distinct from $R$. beebei, as obtained by Murphy et al. (2017). However, both specimens of $R$. humboldti collapse in a polytomy together with the well supported $R$. centralis (JGC and JAF =99\%). Both taxa seem to differ in several morphological characters (Narvaes and Rodrigues, 2009; although these authors considered R. beebei and R. humboldti as a single taxon) and the UPDs between 
the specimens of both species are $1.04 \%-1.37 \%$ (see table 5). The poor internal resolution of this clade could be due to the reduced gene sampling for both specimens of $R$. humboldti (see appendix 2). However, a thorough analysis including additional molecular markers and morphological comparisons with $R$. humboldti s.s. is necessary to test the validity of $R$. centralis.

\section{The Mitochondrial Lineage of Rhinella HORRIBILIS}

The included mitochondrial sequences of Rhinella horribilis together with the $R$. crucifer $+R$. marina Groups constitute a strongly supported clade (JGC and JAF $=100 \%$ ) in the TE analysis. Within this clade, they are recovered as sister taxon of a poorly suported clade (JGC $=56 \%$, $\mathrm{JAF}=72 \%$ ) formed by the two aforementioned groups. Alternatively, this lineage is recovered in the ML analysis as the sister of the $R$. crucifer Group, with low support ( $44 \%$ ultrafast bootstrap support value). This clade is, in turn, sister to the $R$. marina Group (supplementary data 4.3). As we discuss below (see "Hybridization and genetic introgression in Rhinella"), the strong phylogenetic incongruence between mitochondrial and nuclear sequences of all the sampled specimens of $R$. horribilis is the result of a past hybridization with introgression event in which $R$. horribilis incorporated this mitochondrial lineage and completely replaced the original mtDNA of this species. We hypothesize that this mitochondrial lineage corresponds to a still unknown, or perhaps even extinct species of $R h i-$ nella, as we could not associate it to any of the 92 included species. In addition, two well-supported lineages are genetically differentiated within this mitochondrial clade according to the tree topology and proportionately large genetic distances (mean UPD $=4.19 \%$, table 6): one includes most populations of $R$. horribilis from Colombia and Central America, which we associate to $R$. horribilis s.s., whereas the second lineage includes populations of $R$. horribilis from Ecuador that represent an undescribed species (Rhinella sp. 1). This structure is not recovered by the nuclear sequences of $R$. horribilis because they collapse in polytomy.

\section{The RHINELla CRUCIFER Group}

Diagnosis: This species group was recovered as monophyletic and well supported (JGC and JAF $=100 \%$ ), as in previous studies (Maciel et al., 2006; Thomé et al., 2010, 2012). Three phenotypic characters states optimize as synapomorphies of the Rhinella crucifer Group: (1) insertion of the $\mathrm{m}$. extensor digitorum longus on the metatarsophalangeal joint of digiti IV absent (char. 39.0), which is homoplastic in a subclade of the $R$. granulosa Group, in the $R$. margaritifera Clade, and in some of the earlierdiverging bufonids; (2) lateral $\mathrm{m}$. dorsometatarsalis proximalis digiti IV with a discrete tendon inserting on the proximal interphalangeal joint of digiti IV (char. 42.1), with an instance of homoplasy in Nannophryne variegata; and (3) the occurrence of a vertebral line (char. 54.1), with several instances of homoplasy within $R h i$ nella. Other additional character states that could optimize as a synapomorphy of this group or an internal clade, depending on their occurence in $R$. casconi and $R$. henseli, that are still unknown: (1) dorsal protuberance of the illium small, low, and laterally projected (char. 32.1; condition within the group known only in $R$. crucifer); and (2) inguinal fat bodies absent (char. 51.0), with instances of homoplasy in $R$. achavali, $R$. rumbolli, in a subclade of the $R$. festae Group, in the $R$. margaritifera Group, and in several sampled outgroups.

Species of the Rhinella crucifer Group can be distinguished from members of the other groups of Rhinella by the following combination of character states: (1) supraorbital crest well developed (char. 1.2), (2) pretympanic crest weak (char. 2.1), (3) nasal and frontoparietal articulate along most of its margin but not completely (char. 8.2), (4) occipital artery pathway completely covered with bone (char. 10.2), (5) medial ramus of the pterygoid fused medially along approximately half the length of the parasphenoid ala (char. 21.2), (6) head of the m. extensor carpi ulnaris 


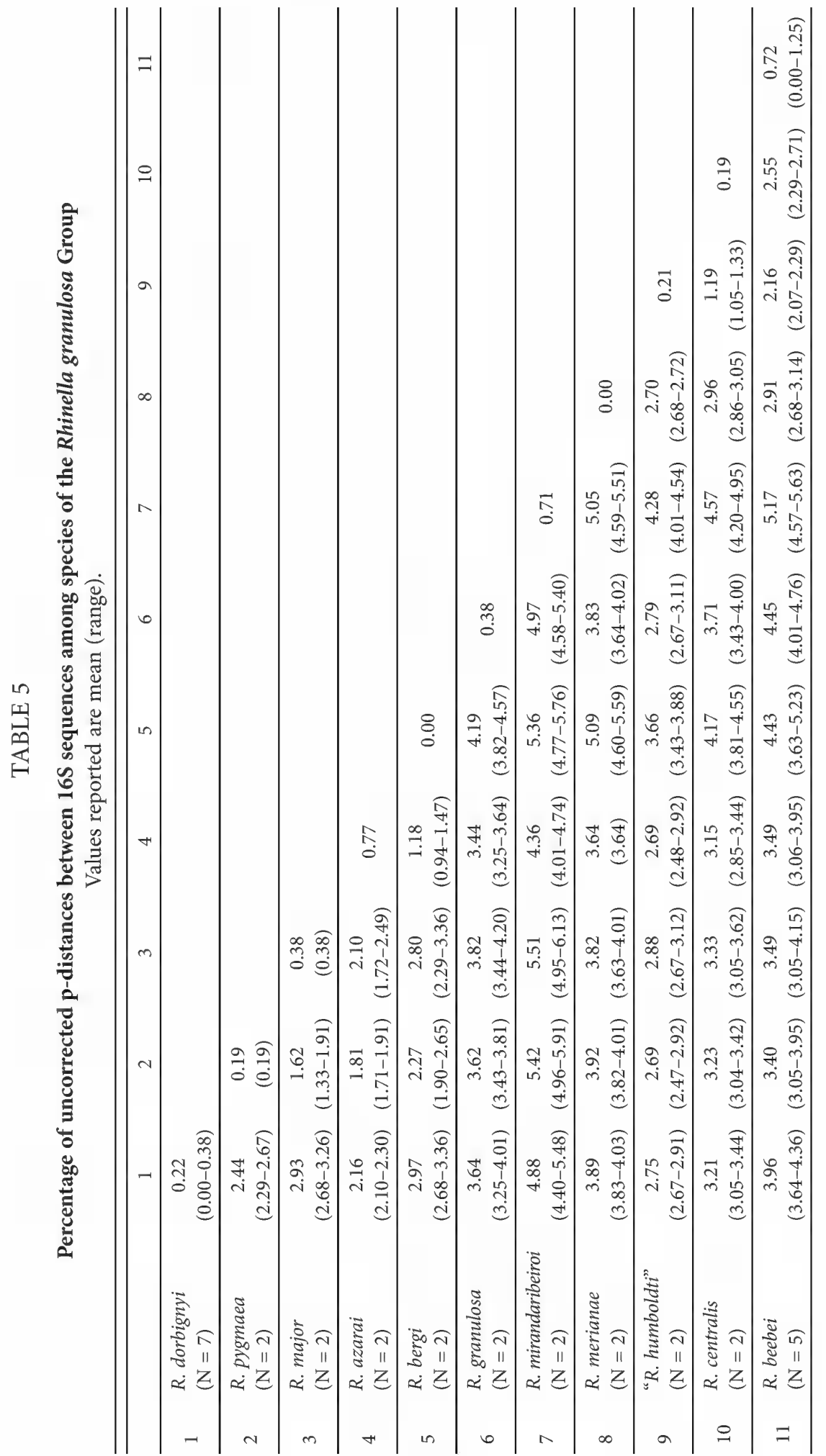




\section{TABLE 6}

\section{Percentage of uncorrected p-distances between $16 \mathrm{~S}$ sequences among terminals of the ghost introgressed mitochondrion \\ Values reported are mean (range).}

\begin{tabular}{cccc}
\hline & & 1 & 2 \\
\hline \multirow{2}{*}{1} & R. horribilis & 0.89 & \\
& $(\mathrm{~N}=4)$ & $(0.19-1.34)$ & \\
\hline \multirow{2}{*}{2} & Rhinella sp. 1 & 4.23 & 0.49 \\
& $(\mathrm{~N}=4)$ & $(3.24-5.98)$ & $(0.00-0.86)$ \\
\hline
\end{tabular}

from the radioulna with an origin via a flat tendon (char. 47.1), (7) parotoid gland approximately ellipsoid (char. 56.0), (8) tarsal fold present (char. 65.1), (9) caudal musculature of larvae uniformly dark except an unpigmented longitudinal stripe along the inferior edge (char. 69.1), and (10) adhesive gland of the embryo divides after opercular fusion (char. 82.2).

Sister ClAdE: The Rhinella marina Group.

CONTENTs (5 SPECIES): Rhinella casconi Roberto et al., 2014; $R$. crucifer (Wied, 1821); $R$. henseli (Lutz, 1934); R. inopina Vaz-Silva et al., 2012; and R. ornata (Spix, 1824) [including R. abei (Baldissera et al., 2004), new synonymy, see below].

Distribution: These species are distributed mainly along the Atlantic Forest region, except $R$. inopina, which inhabits the Cerrado region (Baldissera et al., 2004; Thomé et al., 2010; Arruda et al., 2014; Roberto et al., 2014). See map 4 (available at https://doi.org/10.5531/sd. sp.46) for type localities and sampled localities.

Comments: The general internal relationships among the species are similar to those reported by Thomé et al. (2010, 2012). Previously, Maciel et al. (2006) recovered this group as monophyletic, but the internal relationships among the species were poorly supported. Based on our results and those of previous analyses, we discuss below several relevant taxonomic issues of this group.

Based on external morphology and morphometric analyses, Baldissera et al. (2004) reviewed the taxonomy of Rhinella crucifer. These authors resurrected two species ( $R$. henseli and $R$. ornata) and recognized two new species ( $R$. abei and $R$. pombali) for several populations previously con- sidered within $R$. crucifer. More recently, two additional species were described, $R$. casconi and $R$. inopina (Vaz-Silva et al., 2012; Roberto et al., 2014). Three of these species (i.e., $R$. casconi, $R$. crucifer, and $R$. henseli) were recovered as strongly supported lineages (JAF and JGC = $100 \%)$, and they have moderate UPDs with respect to other species ( $>0.98 \%$ see table 7$)$.

Thomé et al. $(2010,2012)$ found Rhinella abei nested in $R$. ornata and stressed the need for including additional molecular markers before taking a taxonomic decision on this species. Our analyses, considering additional genes, recovered R. abei as nonmonophyletic and nested within $R$. ornata. Moreover, vouchers from multiple localities show no consistent differences in the morphological characters employed by Baldissera et al. (2004) to distinguish these species (e.g., color in preserved specimens, subocular band distinctiveness, head width, and forearm development; M.O.P. and D.B., personal obs.). Thus, we found no evidence to support the distinctiveness of $R$. $a b e i$, and consider Bufo abei Baldissera et al., 2004, a junior synonym of Rhinella ornata (Spix, 1824).

Rhinella ornata (including $R$. abei) is monophyletic, but poorly supported $(\mathrm{JGC}=56 \%, \mathrm{JAF}=$ $58 \%$ ). Its sister taxon is $R$. inopina, a putatively independent lineage (see FCA analysis in Thomé et al., 2012) recovered with strong support (JGC and $\mathrm{JAF}=99 \%$ ) in the TE analysis. The genetic distances between $R$. ornata and $R$. inopina are very low for the $R$. crucifer Group $(0.2 \%-0.7 \%)$ and cannot be attributable to evident mitochondrial introgression (see Thomé et al., 2012; fig. 9); some morphological characters (e.g., adult size, 
the coloration of marks on flanks, cloacae, and the posterior surface of thighs, and the disposition of parotoid macroglands) were proposed to differentiate both species. Considering the exceptionally low UPDs between $R$. ornata and $R$. inopina and the considerably wide range of $R$. ornata, further comparative studies accounting for geographical variation in these characters are necessary to definitely support or reject the status of $R$. inopina as a distinct species.

\section{The RHinella marina Group}

Diagnosis: Our TE analysis recovered a poorly supported Rhinella marina Group (JGC = $63 \%, \mathrm{JAF}=79 \%)$ as in previous studies with less dense taxon sampling (e.g., Maciel et al., 2010; van Bocxlaer et al., 2010; Pyron, 2014). Two phenotypic synapomorphies support this species group: (1) the jagged or scalloped articulation between the medial ramus of pterygoid and parasphenoid alae (char. 22.1), with instances of homoplasy in $R$. atacamensis, $R$. achalensis, $R$. sternosignata, in a subclade of the $R$. festae Group, and in some species of the R. margaritifera Group, and (2) the sacral diapophyses with the anterior edge angled posteriorly to the midline axis of the vertebral column (char. 30.0), with instances of homoplasy in Rentapia hosii and Schismaderma carens.

Species of the Rhinella marina Group can be distinguished from members of the other species groups of Rhinella by the following combination of character states: (1) preorbital crest well developed (char. 0.2), (2) supraorbital crest well developed (char. 1.2), (3) pretympanic crest well developed (char. 2.2), (4) nasal and frontoparietal articulate along the entire margin (char. 8.3), (5) occipital artery pathway completely covered with bone (char. 10.2), (6) medial ramus of the pterygoid fused and extending medially along approximately half the length of the parasphenoid ala (char. 21.2), (7) $\mathrm{m}$. extensor digitorum longus with an insertion on the metatarsophalangeal joint of digiti IV (char. 39.1), (8) inguinal fat bodies present (char. 51.1), (9) parotoid gland approximately ellipsoid, longer than wide or triangular and bulky (char. 56.0 or 56.3),
(10) tarsal fold present (char. 65.1), (11) adhesive gland division after operculum fusion in embryo (char. 82.2), and (12) eggs biserially disposed in strings (char. 87.1).

Sister ClADE: The Rhinella crucifer Group.

Contents (10 species): Rhinella achavali (Maneyro et al., 2004); R. arenarum (Hensel, 1867); $R$. cerradensis Maciel et al., 2007; $R$. diptycha (Cope, 1862) [including R. jimi (Stevaux, 2002), new synonymy, see below]; $R$. horribilis (Wiegmann, 1833); R. icterica (Spix, 1824); $R$. marina (Linnaeus, 1758); $R$. poeppigii (Tschudi, 1845); $R$. rubescens (Lutz, 1925); and $R$. veredas (Brandão et al., 2007).

DisTRIBUTION: These species are naturally distributed throughout all the main regions of the Neotropics, except in arid Andean areas and the Austral Temperate Forest region (Cei, 1980; De la Riva, 2002; Stevaux, 2002; Kwet et al., 2006; Brandão et al., 2007; Maneyro and Kwet, 2008; Santana et al., 2010; Acevedo et al., 2016; Saito et al., 2016; Venâncio et al., 2017). See map 5 (available at https://doi. org/10.5531/sd.sp.46) for type localities and sampled localities.

Comments: Both recovered phenotypic synapomorphies were suggested as distinctive character states of this group by Pramuk (2006). Moreover, Maciel et al. (2010) proposed four osteological synapomorphies for the Rhinella marina Group (ventral ramus of the squamosal ventrolateral in posterior view; anterior extension of the cultriform process extends beyond the orbitonasal foramina; sphenethmoid lightly ossified; medial ramus of the pterygoid relatively narrow) and one skin-secretion compound (occurrence of a specific indolealkylamine). These characters were not considered in our TE analysis and should be reevaluated considering a denser sample of outgroups than the one employed by Maciel et al. (2010).

The finding of a moderately supported $R h i-$ nella marina Group contrasts with previous studies that recovered it well supported (e.g., Maciel et al., 2010; van Bocxlaer et al., 2010; Pyron, 2014; Jetz and Pyron, 2018). Two distinctive moderately supported clades are evident in 


\section{TABLE 7}

Percentage of uncorrected p-distances between $16 \mathrm{~S}$ sequences among species of the Rhinella crucifer Group Values reported are mean (range).

\begin{tabular}{|c|c|c|c|c|c|c|}
\hline & & 1 & 2 & 3 & 4 & 5 \\
\hline 1 & $\begin{array}{l}\text { R. henseli } \\
(\mathrm{N}=3)\end{array}$ & $\begin{array}{c}0.25 \\
(0.00-0.38)\end{array}$ & & & & \\
\hline 2 & $\begin{array}{l}\text { R. casconi } \\
(\mathrm{N}=2)\end{array}$ & $\begin{array}{c}3.62 \\
(3.62)\end{array}$ & $\begin{array}{c}0.00 \\
(0.00)\end{array}$ & & & \\
\hline 3 & $\begin{array}{l}\text { R. crucifer } \\
(\mathrm{N}=2)\end{array}$ & $\begin{array}{c}3.14 \\
(3.04-3.23)\end{array}$ & $\begin{array}{c}1.43 \\
(1.33-1.52)\end{array}$ & $\begin{array}{c}0.19 \\
(0.19)\end{array}$ & & \\
\hline 4 & $\begin{array}{l}\text { R. inopina } \\
(\mathrm{N}=3)\end{array}$ & $\begin{array}{c}3.42 \\
(3.42) \\
\end{array}$ & $\begin{array}{c}1.33 \\
(1.33) \\
\end{array}$ & $\begin{array}{c}0.86 \\
(0.76-0.95) \\
\end{array}$ & $\begin{array}{c}0.25 \\
(0.00-0.38) \\
\end{array}$ & \\
\hline 5 & $\begin{array}{l}\text { R. ornata } \\
(\mathrm{N}=11)\end{array}$ & $\begin{array}{c}3.25 \\
(3.04-3.61)\end{array}$ & $\begin{array}{c}1.30 \\
(1.14-1.52)\end{array}$ & $\begin{array}{c}0.82 \\
(0.57-1.14)\end{array}$ & $\begin{array}{c}0.35 \\
(0.19-0.57)\end{array}$ & $\begin{array}{c}0.29 \\
(0.00-0.76)\end{array}$ \\
\hline
\end{tabular}

this genetically and taxonomically complex species group. The first roughly corresponds to the North-Central Clade of Maciel et al. (2010) and is composed of $R$. diptycha (including $R$. jimi), $R$. horribilis, $R$. marina, $R$. poeppigii, and $R$. veredas, but does not include $R$. cerradensis (although see MP tree in Maciel et al., 2010: fig. 3).

Rhinella poeppigii, $R$. veredas, and R. marina s.s. (see Acevedo et al., 2016) are successive sister taxa of the remaining species of the NorthCentral Clade but their positions are poorly supported (JGC <38\%, JAF <54\%). Except for $R$. veredas, the monophyly of these species are poorly supported $(\mathrm{JGC}=74 \%, \mathrm{JAF}=77$ for $R$. poeppigii; JGC $<25 \%$, JAF $<50 \%$ for $R$. marina). The relationships among $R$. diptycha, R. jimi, and the nuclear sequences of $R$. horribilis and Rhinella sp. 1 are poorly resolved (see fig. 12). The lack of resolution and support for the internal relations of the North-Central Clade could be due, at least in part, to (1) the nuclear markers employed that do not provide sufficiently informative variation to resolve the relationships in the absence of mitochondrial information for some terminals, and (2) the occurrence of putative past and present hybridization that could not be detected with the available molecular evidence.

The occurrence of a deep mitochondrial divergence in Rhinella horribilis and Rhinella sp. 1 precluded the combination of the mitochondrial and nuclear sequences into single terminals. This results in an unstable and poorly supported phylogenetic position for nuclear sequences of both species in the TE analysis (in a polytomy with $R$. jimi specimens). Because the nuclear sequences employed provided relatively few informative characters, their relationships should be better explored considering additional evidence. Moreover, $R$. horribilis diverges in several morphological characters (adult morphology, osteology, and larval morphology; see Savage, 2002; Stevaux, 2002; Kwet et al., 2006; Tolledo and Toledo, 2010; Acevedo et al., 2016) from the largely allopatric $R$. jimi. Otherwise, the position of $R$. jimi in a polytomy together with the specimens of R. diptycha and the very low UPDs (0\%-0.19\%) among the specimens of these species indicate that the taxa are conspecific. Rhinella diptycha and $R$. jimi are two morphologically similar species with large parotoid and tibial macroglands. Remarkably, $R$. jimi has distinctive glands on its forearms and on both sides of the cloaca that were considered as the only distinctive characters from $R$. diptycha (Stevaux, 2002; Kwet et al., 2006). Mailho-Fontana et al. (2018) found that both species have similar types and distribution of skin glands, although in different proportions. These authors proposed that this differential development could be related to the occupancy 
of xeric environments by $R$. jimi. We also found a greater glandular development in the forearms and both sides of the cloaca in some specimens of $R$. diptycha from different localities of the dry Chaco in Argentina (M.O.P. and D.B., personal obs.). Based on these observations, we consider Bufo jimi Stevaux, 2002, a junior synonym of Rhinella diptycha (Cope, 1862). More physiological and histological studies, investigating different populations from different environments, could help to understand the patterns of variation in the development of macroglands in this species.

The other clade of the Rhinella marina Group is composed of $R$. achavali, $R$. arenarum, $R$. cerradensis, $R$. icterica, and $R$. rubescens (fig. 12), and roughly corresponds to the South-Central Clade of Maciel et al. (2010). Within this clade, $R$. arenarum is supported as sister taxon of the remaining species of the clade with strong support (JGC and JAF $=100 \%$ ). The sampled specimen from the populations that had been considered as $R$. arenarum mendocina is nested within the remaining specimens of $R$. arenarum.

The sister clade of Rhinella arenarum is well supported but it is internally poorly resolved. This includes $R$. achavali, $R$. cerradensis, $R$. icterica, and $R$. rubescens. Rhinella cerradensis and $R$. rubescens are reciprocally monophyletic, their UPDs are low $(0.19 \%-0.74 \%$, see table 8$)$, and constitute a strongly supported clade (JGC and $\mathrm{JAF}=99 \%$ ) that collapses in a basal polytomy within the clade. Rhinella achavali was recovered nested in a poorly supported clade (JGC $<25 \%$, JAF <50\%) composed of some populations of $R$. icterica and the UPDs within this clade are low $(0.37 \%-0.76 \%$, see table 8$)$. Although $R$. icterica is quite variable morphologically (M.O.P. and D.B., personal obs.) and this species includes several synonymized forms (e.g., Bufo missionum; Faivovich and Carrizo, 1997), this taxon is divergent morphologically from R. achavali (see Maneyro et al., 2004; Kwet et al., 2006; M.O.P., F.K., and C.B., personal. obs.). Finally, some specimens tentatively assigned to $R$. cerradensis ( $R$. aff. cerradensis) collapse into a basal polytomy within the sister clade of $R$. arenarum. We refrain from taking any decision regarding the taxonomy of these species pending more studies, particularly with respect to understanding the effect of genetic (e.g., nuclear and/or mitochondrial introgressions) and environmental (e.g., phenotypic plasticity) factors on their morphological variation.

\section{The Rhinella margaritifera Clade}

DiAGnOsis: This well-supported clade (JGC and JAF $=99 \%$ ) is diagnosed by two phenotypic synapomorphies: (1) alary process of the premaxillae angled anteriorly to the anterior margin of the pars dentalis of premaxillae (char. 13.2), with instances of homoplasy in Incilius coniferus, Rhinella achalensis, $R$. ornata, $R$. poeppigii, and Schismaderma carens; and (2) skin of dorsum of females with small tubercles lacking cornified tips (char. 52.3).

Sister ClADE: The Rhinella marina Clade.

Contents: Rhinella sternosignata and the $R$. festae, $R$. margaritifera, and $R$. veraguensis Groups.

Distribution: The species of this clade are mainly distributed throughout Amazonia and montane humid forest of the Andes. Some species of this clade are also found in the Atlantic Forest, Caatinga, Cerrado, Chaco/Pantanal, and Chocó regions, and in Central America (Duellman, 1999).

Comments: Within this clade, Rhinella sternosignata is recovered as the sister taxon of a large, poorly supported clade (JGC $=49 \%$, JAF $=71 \%)$. This last clade is supported by a single phenotypic synapomorphy (ventral ridges on the palatine absent; char. 20.0), which is homoplastic in several species of the $R$. marina Group and outgroups. The clade is composed of three strongly supported species groups (JGC and JAF $=100 \%)$ : (1) the redefined $R$. veraguensis Group, (2) the redefined R. festae Group, and (3) the redefined $R$. margaritifera Group. The R. festae and R. margaritifera Groups were recovered as sister clades with moderate support $(\mathrm{JGC}=81 \%, \mathrm{JAF}=89 \%)$ and five character states optimize as phenotypic synapomorphies 
of this clade: (1) discrete superficial cutaneous tendons absent (char. 33.0); (2) lateral slip of the $\mathrm{m}$. interphalangeus proximalis digiti $\mathrm{V}$ (foot) absent, with instances of homoplasy in $R$. major and $R$. papillosa (char. 35.0); (3) $\mathrm{m}$. abductor brevis plantaris hallucis absent (char. 36.0), with instances of homoplasy in Anaxyrus woodhousii, Peltophryne empusa (polymorphic), and $R$. mirandaribeiroi; (4) slip of the medial $\mathrm{m}$. lumbricalis brevis digiti $\mathrm{V}$ originating from the distal carpal 3-4-5 absent (char. 43.0) with an instance of homoplasy in Nannophryne variegata (polymorphic); and (5) head of the m. extensor carpi ulnaris from the radioulna with a fleshy origin (char. 47.0), with an instance of homoplasy in P. empusa. A similar topology for the main internal clades of the $R$. margaritifera Clade was recovered in the ML analysis (supplementary data 4.4-4.5).

\section{RHINELLA STERNOSIGNATA}

DiAgnosis: Rhinella sternosignata (Günther, $1858 \mathrm{~b}$ ) was recovered as the sister taxon of all other species of the R. margaritifera Clade, with poor support $(\mathrm{JGC}=49 \%$, JAC $=71 \%)$. Phenotypic autapomorphies are: (1) acuminate anterior margins of nasals (char. 6.1), with instances of homoplasy in Incilius coniferus and the $R$. margaritifera Group; (2) articulation of the zygomatic ramus of the squamosal with the maxilla (char. 15.1), with instances of homoplasy in the R. granulosa Group and Peltophryne lemur; (3) articulation between the medial ramus of the pterygoid and parasphenoid alae with a jagged suture (char. 22.1) with instances of homoplasy in $R$. achalensis, $R$. atacamensis, some species of the $R$. festae and $R$. margaritifera Groups, and in the $R$. marina Group; (4) parotoid gland round to ovoid mostly symmetrical (char. 56.2); (5) large size of adult males with respect to adult females (char. 66.1), with instances of homoplasy in $R$. yanachaga, and in several species of the $R$. marina Clade; and (6) unpigmented eggs (char. 88.0), with instances of homoplasy in Ansonia longidigita, Rhinella justinianoi, R. stanlaii, and in the R. festae Group.
Distribution: This species inhabits montane forests of the Cordillera de la Costa and the Andean Cordillera de Mérida of Venezuela (La Marca and Mijares-Urrutia, 1996; BarrioAmorós et al., 2019). See map 5 (available at https://doi.org/10.5531/sd.sp.46) for type and sampled localities.

Sister ClADE: The clade composed of the Rhinella festae, R. margaritifera, and $R$. veraguensis Groups.

Comments: This species was tentatively associated with the Rhinella margaritifera (Cei, 1972a; Hoogmoed, 1990; Duellman and Schulte, 1992) or R. granulosa Groups (Gallardo, 1962). Pereyra et al. (2016a) rejected the inclusion of this species in any of these groups, but they could not determine its relationships rigorously due to the poor sampling of Rhinella. This species was wrongly reported for many localities outside the Cordillera de la Costa montane forests region in Venezuela as discussed by La Marca and Mijares-Urrutia (1996). Vélez-Rodríguez (1999) recorded this species in error for Colombia (see Vélez-Rodríguez, 2004b, 2005). Additionally, there are a large number of recent reports of $R$. sternosignata for Colombia (Acosta-Galvis et al., 2006; Romero et al., 2008; Acosta-Galvis, 2012a, 2012b). Analyzed specimens tentatively assigned to this species from the eastern slope of the Cordillera Oriental in Colombia (MAR 1314, Boyacá and MAR 1955, Caquetá) were unrelated to the specimen of $R$. sternosignata from Venezuela in the phylogenetic analyses, and instead, they represent an undescribed species along with other specimens of the R. margaritifera Group from Loreto, Peru, and Miranda, Venezuela (Rhinella sp. 13, see below). These results, and the absence of comprehensive comparative studies considering topotypical material of $R$. sternosignata, indicate that there is no evidence to consider its occurrence in Colombia.

\section{The Rhinella Veraguensis Group}

Diagnosis: No phenotypic synapomorphies were recovered for this strongly supported group (JGC and JAF $=100 \%$ ). This is mainly due to the lack of detailed information for one of its two con- 


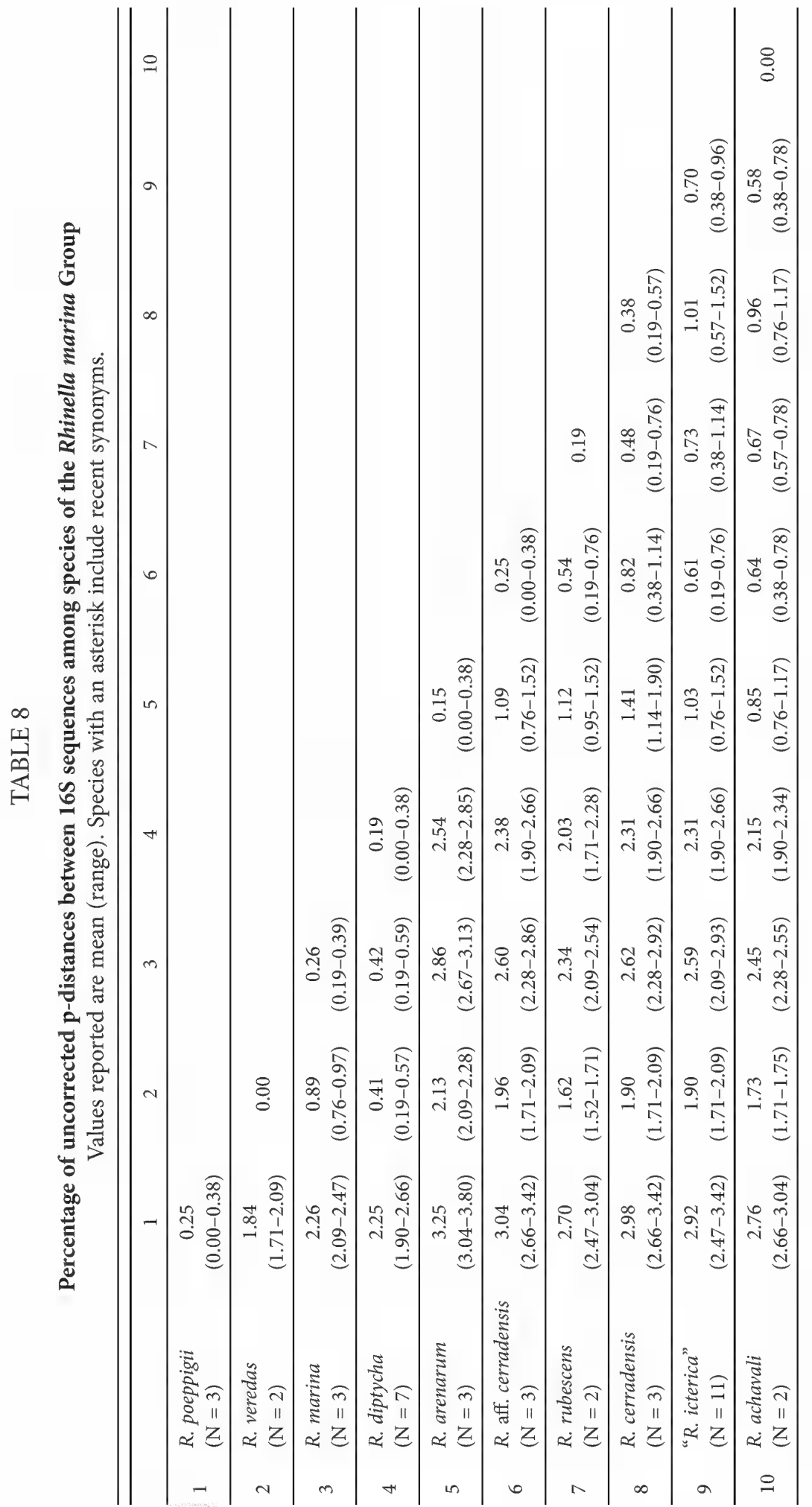


stituent clades (composed of Rhinella sp. 2 [see below], $R$. inca and $R$. leptoscelis). Nevertheless, some character states might represent synapomorphies for this group or a subclade: (1) the articulation of jaw opposite to the fenestra ovalis (char. 16.1), with instances of homoplasy in Nannophryne variegata, Rhinella beebei, $R$. merianae, $R$. yanachaga, and the $R$. arunco Group; (2) lightcolored nuptial pads (char. 62.0); (3) larval peribranchial region with bulging regions lateral to the oral disc (char. 67.1); (4) larval oral disc with complete A2 labial tooth row (char. 73.0), with instances of homoplasy in Amazophrynella aff. minuta, Ansonia longidigita, Melanophryniscus gr. stelzneri, Phrynoidis juxtaspera, and Schismaderma carens; (5) the dextral opening of the vent tube (char. 75.1); and (6) eggs laid in open clumps (char. 86.1; structure of the spawn only known in $R$. rumbolli within the $R$. veraguensis Group).

The species of the Rhinella veraguensis Group can be distinguished from members of the other species groups of Rhinella by the following combination of character states: (1) preorbital crest weak (char. 0.1), (2) supraorbital crest weak (char. 1.1), (3) pretympanic crest weak (char. 2.1), (4) medial ramus of the pterygoid fused and extending medially along approximately half the length of the parasphenoid ala (char. 21.2), (5) m. extensor digitorum longus without an insertion on the metatarsophalangeal joint of digiti IV (char. 39.0), and (6) tarsal fold absent (char. 65.0).

Sister ClAde: The clade composed of the Rhinella festae and $R$. margaritifera Groups.

CONTENT (9 SPECIEs): Rhinella chrysophora (McCranie et al., 1989); R. fissipes (Boulenger, 1903); R. gnustae (Gallardo, 1967); R. inca (Stejneger, 1913); R. justinianoi (Harvey and Smith, 1994); R. leptoscelis (Boulenger, 1912); R. quechua (Gallardo, 1961) [including R. amboroensis (Harvey and Smith, 1993), new synonymy, see below]; R. rumbolli (Carrizo, 1992); and $R$. veraguensis (Schmidt, 1857).

Distribution: All species of the Rhinella veraguensis Group are distributed in Andean humid forests of Argentina, Bolivia, and Peru, except $R$. chrysophora, which inhabits the Central American Atlantic moist forests in Honduras (Rodríguez et al., 1993; De la Riva et al., 2000; Köhler, 2000; Lavilla and Cei, 2001; Padial et al., 2009; McCranie, 2017). See map 6 (available at https://doi.org/10.5531/sd.sp.46) for type localities and sampled localities.

Comments: The former Rhinella veraguensis Group (see appendix 5) is recovered as polyphyletic. To remedy this, we restrict its content to the clade of species most closely related to $R$. veraguensis. This group also comprises two species not included in the phylogenetic analyses that share several putative synapomorphies with the species sampled here (see below). Two clades were recovered within this redefined $R$. veraguensis Group. One clade is poorly supported (JGC = $67 \%$, JAF $=76 \%)$ and comprises the southernmost distributed species of the group. In the second clade, we were unable to examine the voucher of R. amboroensis (MNK 5302), but this specimen was collected near the type locality of the species. The specimen was recovered as the sister taxon of $R$. quechua and the genetic distance between the specimens is $0 \%$ (see table 9). Both species are very similar morphologically and only a few morphological characters were proposed to differentiate the taxa (i.e., the extension of the foot webbing, ventral skin texture, and finger length). However, these difference are not consistently observed in specimens collected in the type locality of R. amboroensis (I.D.L.R., personal obs.) and they could simply represent variations within $R$. quechua. For these reasons, we consider Bufo amboroensis Harvey and Smith, 1993, a junior synonym of Rhinella quechua (Gallardo, 1961).

The other clade in this group is strongly supported (JGC and JAF $=100 \%$ ) and includes the northernmost distributed species, $R$. inca, $R$. leptoscelis, and an undescribed species from Oxapampa, Peru (Rhinella sp. 2). The UPDs among these three species are relatively low (1.16\%$1.90 \%$; see table 9 ).

Rhinella chrysophora and R. gnustae, two species not included in the phylogenetic analysis, 
are considered to belong to this species group. Rhinella chrysophora is known only from two localities in northern Honduras and is supposedly extinct, not collected since 1996 (McCranie and Castañeda, 2005; McCranie, 2017). This species was originally described as belonging to a distinct genus (Atelophryniscus; McCranie et al., 1989) of no evident relationships within Bufonidae. Pramuk and Lehr (2005), based on a morphological phylogenetic analysis, demonstrated that it is related to the species of the $R$. veraguensis Group s.l. Unfortunately, the character scores for $R$. chrysophora are not available and the condition of the double-stained specimen used in that study is very poor (J.J.O.-S., personal obs.). However, morphological evidence indicates that $R$. chrysophora belongs to the $R$. veraguensis Group, as it posses all its known putative synapomorphies (except for oviposition mode, which is unknown; McCranie et al., 1989; Lavilla and de Sá, 2001; Pramuk and Lehr, 2005).

Rhinella gnustae (Gallardo, 1967) was described based on a single subadult specimen from an imprecise locality of Jujuy Province (Argentina) (Gallardo, 1967; Cei, 1980; Lavilla and Cei, 2001; Lavilla et al., 2002). We tentatively assign this species to the $R$. veraguensis Group based on a combination of characters (although none of them synapomorphic) that occur in multiple species of this group: row of dorsolateral tubercles in the skin absent, tarsal fold absent, and small tubercles without a cornified tip.

\section{The Rhinella festae Group}

Diagnosis: This well-supported group (JGC and $\mathrm{JAF}=100 \%$ ) is diagnosed by the following five phenotypic synapomorphies: (1) skull lightly exostosed (char. 9.1), with instances of homoplasy in Rhinella achavali, $R$. rubescens, the $R$. spinulosa Group, and in several outgroups; (2) fusion of medial ramus of pterygoid with anterolateral margin of the parasphenoid ala (char. 21.1), with instances of homoplasy in Rhinella sp. 12, in the $R$. arunco Group, in some species of the $R$. spinulosa Group, and in several outgroups; (3) anterior margin of cultriform process of parasphenoid truncated (char. 23.2); (4) arboreal habits (char. 84.1) that revert in an internal clade of this group, and with instances of homoplasy in Incilius coniferus and Rentapia hosii; and (5) unpigmented eggs (char. 88.0), with instances of homoplasy in Ansonia longidigita, Rhinella justinianoi, R. stanlaii, and $R$. sternosignata. Other putative synapomorphies of this group or an internal clade are: (1) additional origin of the $\mathrm{m}$. interosseus cruris from the tibiale absent (char. 38.0; known within the group only for $R$. paraguas); (2) $\mathrm{m}$. extensor brevis medius hallucis absent (char. 41.0; known within the group only for $R$. paraguas); and (3) the terrestrial oviposition (char. 85.1; known within the group only in $R$. tacana). Moreover, species of the $R$. festae Group can be distinguished from members of the other species groups of Rhinella by the following combination of character states: (1) preorbital crest absent or indistinguible (char. 0.0), (2) supraorbital crest weak (char. 1.1), (3) discrete superficial cutaneous tendons absent (char. 33.0), (4) lateral slip of the m. interphalangeus proximalis digiti $\mathrm{V}$ absent (char. 35.0), (5) m. abductor brevis plantaris hallucis absent (char. 36.0), (6) $\mathrm{m}$. extensor digitorum longus without an insertion on the metatarsophalangeal joint of digit IV (char. 39.0), (7) slip of the medial $\mathrm{m}$. lumbricalis brevis digiti $\mathrm{V}$ originating from the distal carpal 3-4-5 absent (char. 43.0), (8) head from the radioulna of the $\mathrm{m}$. extensor carpi ulnaris with a fleshy origin (char. 47.0), (9) nuptial pads dark colored (char. 62.1), and (10) tarsal fold absent (char. 65.0).

Sister ClAde: The Rhinella margaritifera Group.

Contents (18 Species): Rhinella acrolopha (Trueb, 1971); R. arborescandens (Duellman and Schulte, 1992); R. chavin (Lehr et al., 2001); $R$. festae (Peracca, 1904); $R$. lilyrodriguezae Cusi et al., 2017; R. lindae (Rivero and Castaño, 1990); R. macrorhina (Trueb, 1971); R. manu Chaparro et al., 2007; R. multiverrucosa (Lehr et al., 2005); $R$. nesiotes (Duellman and Toft, 1979); R. nicefori (Cochran and Goin, 1970); R. paraguas Grant and Bolívar-G., 2014; R. rostrata (Noble, 1920); $R$. ruizi (Grant, 2000); R. tacana (Padial et al., 2006); $R$. tenrec (Lynch and Renjifo, 1990); R. truebae 


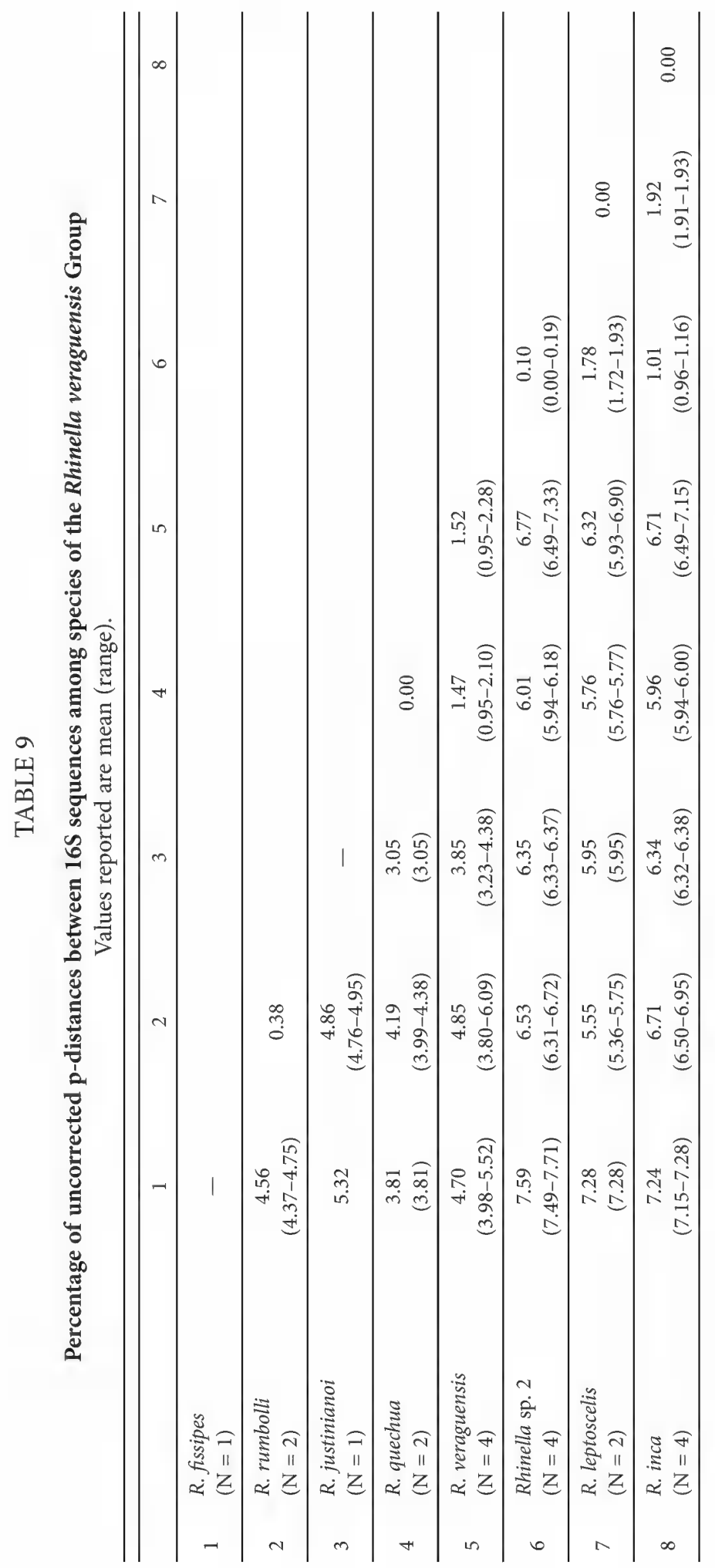


(Lynch and Renjifo, 1990); and R. yanachaga Lehr et al., 2007.

Distribution: Mainly distributed in Andean humid forests of Bolivia, Colombia, Ecuador, and Perú (Trueb, 1971; Duellman and Lynch, 1988; Lynch and Renjifo, 1990; Duellman and Schulte, 1992; Ruiz-Carranza et al., 1996; Lehr et al., 2001, 2005, 2007; Rueda-Almonacid et al., 2004; Chávez et al., 2013; Grant and Bolivar-G., 2014; Cusi et al., 2017). The only species distributed outside this region is Rhinella acrolopha, which inhabits the Chocó region (Darién, Panama; Trueb, 1971). See maps 7 and 8 (available at https://doi.org/10.5531/sd.sp.46) for type localities and sampled localities.

Comments: Analyses of our expanded sample of the Rhinella acrolopha Group corroborated the monophyly of that group; however, they also corroborated its placement among a subset of the species referred to the $R$. veraguensis Group s.l. Insofar as those species are paraphyletic with respect to the $R$. acrolopha Group, the only means of maintaining the current composition of the R. acrolopha Group would be to recognize two or more groups within the larger clade, which we believe to be unwarranted at this time. Consequently, we redefined the R. festae Group as was originally proposed by Moravec et al. (2014) to also include all the species previously referred to the $R$. acrolopha Group and three species ( $R$. arborescandens, $R$. multiverrucosa, and $R$. tacana) of the former $R$. veraguensis Group.

Pramuk (2006) proposed a close phylogenetic relationship between Rhamphophryne and some species of the Rhinella veraguensis Group as formerly defined, but she did not provide a diagnosis for the inclusive clade. As defined in this study, the R. festae Group is a morphologically and ecologically diverse clade of toads; the lack of information for many aspects of these species is remarkable (e.g., adult osteology, adult musculature, larval morphology, natural history; see below).

Species of this group have notably high interspecific UPDs compared to species of other groups of Rhinella (see tables 3-11) except between the pairs $R$. chavin/R. multiverrucosa and $R$ nicefori/R. ruizi. Sequences of the included specimen assigned to $R$. multiverrucosa (MUBI 11455 ) are identical (UPD $=0 \%$ ) to the topotype of $R$. chavin (sequence DQ158441 from Pramuk, 2006). Although morphologically most similar to R. multiverrucosa, the specimen MUBI 11455 was not collected near the type locality of this species and was actually collected closer to the type locality of $R$. chavin (see map 8; available at https://doi.org/10.5531/sd.sp.46). Most of the characters that distinguish these two species involve differences in glandular development. Our results should be tested considering the existing morphological variation within $R$. chavin and including topotypes of $R$. multiverrucosa in a future revision of these species.

Similarly, the UPDs between the included specimen of Rhinella nicefori and topotypic specimens of $R$. ruizi is low (UPDs $=0.19 \%$; see table $10)$. The two species were not explicitly differentiated in the original description of $R$. ruizi (Grant, 2000), but they differ in some characters (degree of cranial ornamentation, the occurrence of vocal slits in adult males, adult size). As we did not sample topotypical material of $R$. nicefor $i$ and cannot discard the occurrence of some additional variation in the diagnostic characters that differentiate the two species, the identity of the included specimen MHUA 4793 should be reevaluated. For this reason, we refrain from taking a taxonomic action, pending a detailed taxonomic evaluation of both species, considering topotypical material of $R$. nicefori and comparison with type specimens.

Two undescribed species within this species group are recovered in our TE analysis. Firstly, some specimens tentatively assigned to Rhinella manu from Madre de Dios and Cusco display high UPDs (3.37\%) with respect to specimens of $R$. manu s.s., suggesting they might represent an undescribed species (Rhinella sp. 3). Second, the specimen of $R$. sp. "gr. acrolopha" (referred to Rhinella sp. C. by Machado et al., 2016) from Caldas (Colombia) is recovered as sister species of $R$. paraguas, and the genetic distance between 
them (UPDs $=5.73 \%-6.11 \%)$ is consistent with the hypothesis that it is an undescribed species (Rhinella sp. 4).

We could not include Rhinella rostrata and $R$. truebae in our analyses. Nevertheless, these species can be placed in the $R$. festae Group on the basis of several character states that are synapomorphies of this group or its internal clades: (1) skull lightly exostosed (char. 9.1); (2-4) columella, annulus tympanicus, and tympanic membrane absent (chars. 48.0, 49.0, and 50.0) in $R$. rostrata (present in $R$. truebae); and (5) finger webbing present (char. 63.1).

\section{The RhINella margaritifera Group}

DiAgNosis: No phenotypic synapomorphies were recovered in our TE analysis for this wellsupported species group (JGC and JAF $=100 \%$ ). However, given the lack of information (see Comments on the phenotypic evidence considered for Rhinella section) for its earlier diverging species (e.g., R. ocellata, R. yunga, and Rhinella sp. 5) or closely related clades (i.e., $R$. sternosignata, the $R$. festae and $R$. veraguensis Groups), the inclusion of additional observations in the phenotypic dataset could provide diagnostic synapomorphies for this clade. A putative synapomorphy for this species group (unknown condition in Rhinella sp. 5) is the acuminate anterior margins of nasals (char. 6.1 ), with instances of homoplasy in Incilius coniferus and R. sternosignata. Moreover, species of the $R$. margaritifera Group can be distinguished from members of the other species groups of Rhinella by the following combination of character states: (1) preorbital crest weak (char. 0.1), (2) medial ramus of the pterygoid fused and extending medially along approximately half the length of the parasphenoid ala (char. 21.2), (3) posterior lobe of the anterolateral process of hyoid absent (char. 25.0), (4) discrete superficial cutaneous tendons absent (char. 33.0), (5) lateral slip of the m. interphalangeus proximalis digiti $\mathrm{V}$ absent (char. 35.0), (6) $\mathrm{m}$. abductor brevis plantaris hallucis absent (char. 36.0), (7) m. extensor digitorum longus without an insertion on the metatarsophalangeal joint of digiti IV (char. 39.0), (8) slip of the medial $\mathrm{m}$. lumbricalis brevis digiti $\mathrm{V}$ originating from the distal carpal 3-4-5 absent (char. 43.0), (9) head of the m. extensor carpi ulnaris from the radioulna with a fleshy origin (char. 47.0), (10) inguinal fat bodies absent (char. 51.0), (11) tarsal fold absent (char. 65.0), and (12) submarginal papillae in the oral disc of larvae absent (char. 71.0).

Sister ClADE: The Rhinella festae Group.

CONTENTS (17 SPECIES): Rhinella acutirostris (Spix, 1824); R. alata (Thominot, 1884); R. castaneotica (Caldwell, 1991); R. cristinae (VélezRodríguez and Ruiz-Carranza, 2002); $R$. dapsilis (Myers and Carvalho, 1945) [including R. gildae Vaz-Silva et al., 2015, new synonymy, see below]; R. hoogmoedi Caramaschi and Pombal, 2006; R. iserni (Jiménez de la Espada, 1875) [including R. yunga Moravec et al., 2014 new synonymy, see below]; $R$. lescurei Fouquet et al., 2007a; R. magnussoni Lima et al., 2007; R. margaritifera (Laurenti, 1768) [including R. martyi Fouquet et al., 2007a, new synonymy, see below]; R. ocellata (Günther, 1858b); R. proboscidea (Spix, 1824); $R$. roqueana (Melin, 1941); R. scitula (Caramaschi and Niemeyer, 2003) [including $R$. paraguayensis Ávila et al., 2010, new synonymy, see below]; $R$. sclerocephala (Mijares-Urrutia and Arends, 2001); R. sebbeni Vaz-Silva et al., 2015; and $R$. stanlaii (Lötters and Köhler, 2000).

Distribution: Mainly distributed in Amazonia, but a few species also occur in the Andes, Atlantic Forest, Caatinga, Cerrado, Chocó, Chaco/ Pantanal, and in Central America (Hoogmoed, 1986, 1990; Ruiz-Carranza et al., 1996; Caramaschi and Pombal, 2006; Köhler et al., 2006; Fouquet et al., 2007a; Moravec et al., 2014; Sugai et al., 2014; Santos et al., 2015; Ávila et al., 2018; Freitas et al., 2018; Silva et al., 2018). See maps 9 and 10 (available at https://doi.org/10.5531/sd.sp.46) for type localities and sampled localities.

Comments: This species group is particularly controversial regarding its diagnosis, content, and taxonomy of its species. The main revisions 


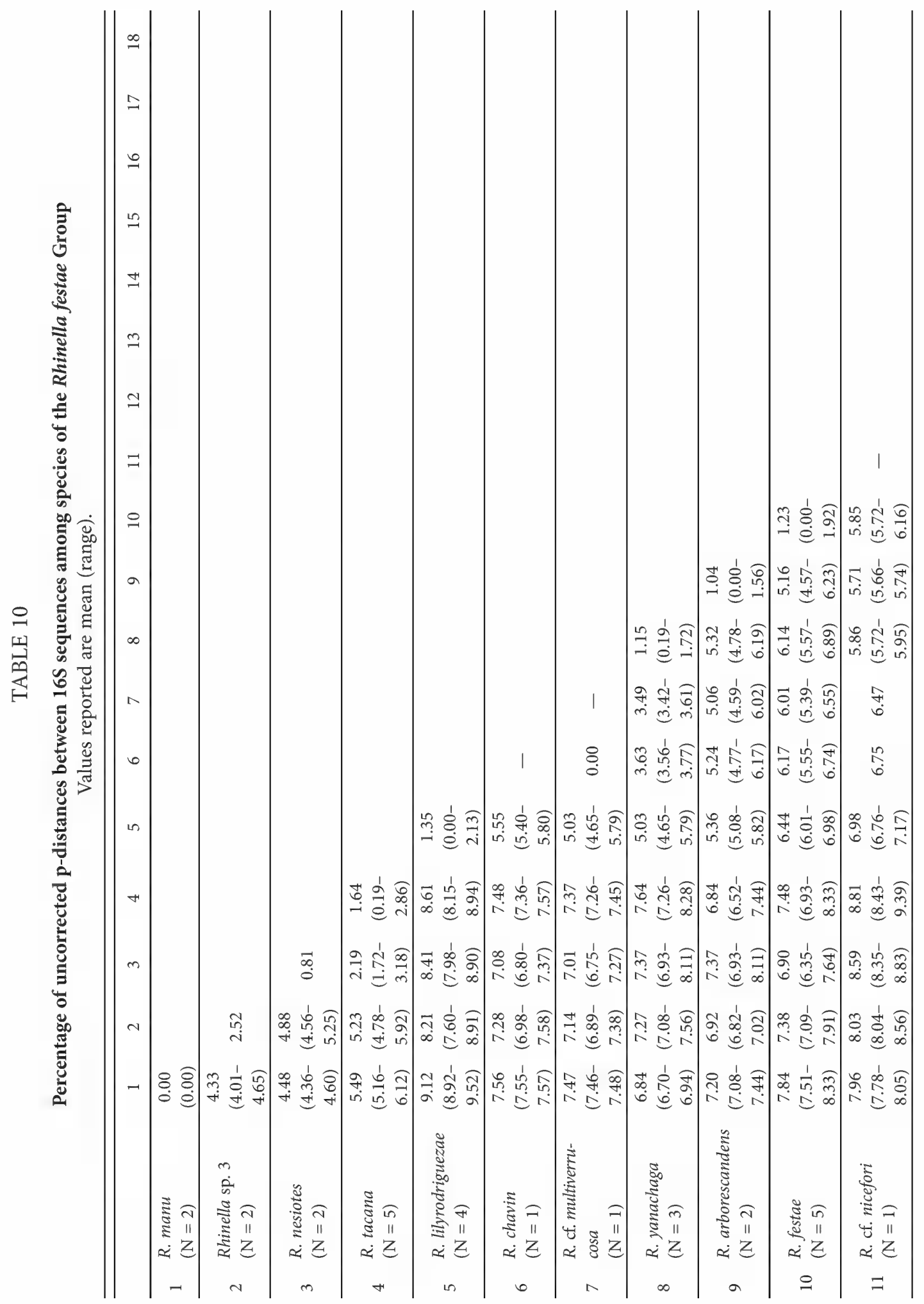




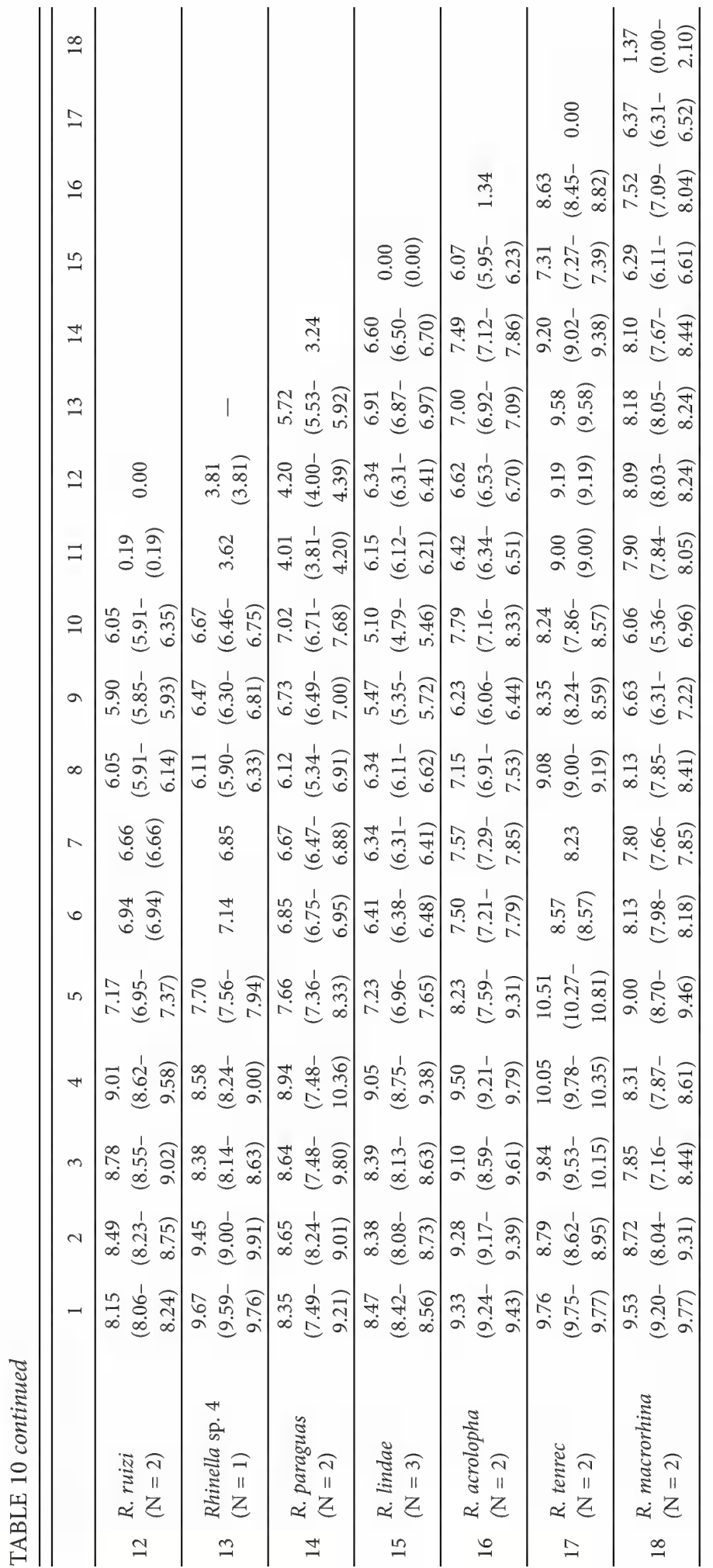


dealing with this group (e.g., Hoogmoed, 1986, 1990; Duellman and Schulte, 1992; Vélez-Rodríguez, 2004b; Pramuk, 2006; Fouquet et al., 2007a) disagreed with respect to the inclusion of multiple species (e.g., Rhinella cristinae, $R$. iserni, $R$. ocellata; see appendix 5). Vélez-Rodríguez (2004b) performed a phylogenetic analysis of the group based on morphological characters and proposed the restriction of its content to a clade diagnosed by two synapomorphies: (1) $\mathrm{m}$. depressor mandibulae composed of two slips with independent origins, on the posterior portion of the otic ramus of the squamosal and the anterior portion of the otic ramus of the squamosal and tympanic annulus; and (2) thickening of the ventral margin of the quadratojugal (our char. 24.2). This redefinition of the $R$. margaritifera Group was not supported by the combined (i.e., molecular + morphological characters) phylogenetic analysis of Pramuk (2006). Pramuk (2006) recovered two synapomorphies for the few exemplar species of this group that she included: (1) the expansion of the posterior ramus of the pterygoid and (2) the occurrence of a lateral articulation between the nasals and the preorbital processes of the maxillae (homoplastic). She also found $R$. ocellata to be the sister species of the $R$. margaritifera Group.

Subsequent studies dealing with the taxonomy of this group (e.g., Fouquet et al., 2007a; Ávila-Pires et al., 2010; Lavilla et al., 2013; Vaz-Silva et al., 2015; Avila et al., 2018) did not explicitly follow a definition based on synapomorphies and thus its composition varied. Based on our results, we redefine the Rhinella margaritifera Group to include the species listed above that are now grouped exclusively on molecular evidence (although some character states may result in putative synapomorphies, see Diagnosis above). Also, the characters proposed by Vélez-Rodríguez (2004) and Pramuk (2006) should be reevaluated considering relevant species not included in these studies (e.g., $R$. sternosignata, $R$. yunga, and Rhinella sp. 5) to understand their polarity in the context of our results.
The lack of a precise type locality and reference specimens, the large intraspecific (including sexual dimorphism) and interspecific variation in adult size and cranial crest shape and development, and the occurrence of sympatry among some species of the Rhinella margaritifera Group turned its taxonomy chaotic and confusing (Hoogmoed, 1989; 1990; Lavilla et al., 2013). A detailed revision of this complex species group is beyond the scope of the present study. As a result, we have been cautious to take taxonomic actions only when evidence is decisive.

The nominal species of the group was described by Laurenti (1768) based on illustrations of Seba (1734) of a specimen from "Brasilia" as the type locality. The identity of this taxon remains unclear after more than two and a half centuries (see discussions in Hoogmoed, 1989; Vélez-Rodríguez, 2004; Fouquet et al., 2007a; Ávila-Pires et al., 2010; Lavilla et al., 2013, 2017). Ávila-Pires et al. (2010) designated the specimen depicted in Seba (1734: pl. 71 , figs. 6,7 ) as the lectotype of Rana margaritifera Laurenti, 1768, and considered the species to be conspecific with Rhinella martyi Fouquet et al., 2007a. Subsequently, Lavilla et al. (2013) invalidated the lectotype designation by Ávila-Pires et al. (2010) and, assuming that the type specimen of $R$. margaritifera was lost, designated and described a neotype for this species. More recently, Lavilla et al. (2017) noted that a previous publication (Milto and Barabanov, 2011) had reported the existence of the type of $R$. margaritifera, invalidating the neotype.

Milto and Barabanov (2011) mentioned two specimens (ZISP 257.1 and 257.2) within the type series of $R$. margaritifera without additional comments. Photographs of both specimens are inadequate to determine which one was used in the illustration of Seba (1734; or if both were used) because both are adult females that fully agree with the description and illustrations. Consequently, it is reasonable to consider the specimens found by Milto and Baravanov (2011) to indeed be those used by Seba (1734) and to arbi- 
trarily designate the specimen ZISP 257.1 as lectotype of Rana margaritifera Laurenti, 1768.

Additionally, we follow Ávila-Pires et al. (2010) regarding the conspecificity of Rhinella margaritifera and $R$. martyi because the lectotype and paralectotype of $R$. margaritifera match almost all the characters used by Fouquet et al. (2007a) to differentiate $R$. martyi from other species of the group (heel extension with hind limbs adpressed and iris coloration unknown in the types of $R$. margaritifera). Thus, we consider $R h i$ nella martyi Fouquet et al., 2007a, to be a junior synonym of $R$. margaritifera (Laurenti, 1768).

Having established the identity of Rhinella margaritifera, we now introduce our results regarding this species group. An undescribed species from Pastaza (Ecuador), Rhinella sp. 5, is recovered with low support $(\mathrm{JGC}=28 \%$, JAF $=58 \%)$ as the sister taxon of all other species of the clade. The sister group of next most inclusive clade is poorly supported (JGC $=32 \%$, JAF $=60 \%$ ) and composed of two morphologically and geographically divergent species, $R$. yunga and $R$. ocellata. Rhinella yunga was recently described from the montane forest of the Selva Central, Peru. Distinctive characters used to diagnose this species in the original description are also present in the poorly known $R$. iserni (skin of dorsum mostly smooth, degree of development of cranial crest, and especially the absence of all the structures of the tympanic middle ear; Jiménez de la Espada, 1875; Moravec et al., 2014; Hoogmoed, personal commun.; J.M. and M.O.P., personal obs.). In addition to their morphological resemblance, both species were described from nearby type localities from the Peruvian Yungas region. Thus, we consider Rhinella yunga Moravec et al., 2014, to be a junior synonym of Rhinella iserni (Jiménez de la Espada, 1875).

Rhinella magnussoni, $R$. cf. margaritifera from Amazonas (Colombia), specimens of " $R$. proboscidea" from Ecuador and Peru, and an undetermined specimen of the $R$. margaritifera Group from São Pedro (Amazonas, Brazil) compose a well-supported clade $($ JGC $=93 \%$, JAF $=94 \%)$.
Rhinella magnussoni and $R$. cf. margaritifera from Amazonas (Colombia) have a relatively high UPD (2.10\%), which seem to support the specific distinctiveness of the latter (Rhinella sp. 6 ). The results of the phylogenetic analysis (see fig. 14) and UPDs among clades (2.60\%-10.27\%; see table 11) strongly suggest that the specimen from São Pedro and both populations of " $R$. proboscidea" from Ecuador (Sucumbios) and Peru (Loreto) correspond to three undescribed species (Rhinella sp. 7-9). The similarity of these undescribed taxa with the phylogenetically distantly $R$. proboscidea s.s. and $R$. castaneotica (see above) indicates the need for a thorough revision of the " $R$. proboscidea" complex.

Another well-supported clade (JGC and JAF = 99\%) includes Rhinella acutirostris, $R$. alata, $R$. sclerocephala, R. sternosignata from Colombia, and several apparently undescribed species (see below). A nonmonophyletic Rhinella alata (sensu Santos et al., 2015) and R. sclerocephala constitute a wellsupported subclade within this clade (JGC $=96 \%$, JAF $=97 \%$ ). The latter species was recovered as sister taxon of the Ecuadorian populations of $R$. alata with poor support $(\mathrm{JGC}=61 \% \mathrm{JAF}=62 \%)$, and their UPDs are $1.31 \%-1.52 \%$. Otherwise, the populations of Panama and Colombia were recovered as a poorly supported lineage (JGC $=55 \%$, $\mathrm{JAF}=58 \%$ ) with UPDs of $0.56 \%-1.52 \%$ with respect to its sister clade. Santos et al. (2015) did not find any morphological character or evident genetic differentiation between the populations from Panama and those from Ecuador as to consider them as different taxa. Otherwise, $R$. sclerocephala was differentiated from $R$. alata by several characters such as its larger size, presence of welldeveloped cranial crests, vertebral apophysis, and bony knob (Mijares-Urrutia and Arends, 2001; Santos et al., 2015), although these characters vary extensively within species of the $R$. margaritifera Group and might be associated with particular environmental conditions over their areas of distribution (see Kutrup et al., 2006; Bandeira et al., 2016). The absence of evident differences between specimens of both clades of $R$. alata, the nested position of $R$. sclerocephala within this clade, and 
the low UPDs seem to support their conspecificity, but we refrain from synonymyzing both taxa due to the low support for the nested position of the specimen of $R$. sclerocephala and the several morphological differences. Nevertheless, it is evident that a detailed taxonomic revision of both taxa considering additional specimens and molecular evidence is required to better resolve the taxonomy within this clade.

The other subclade includes Rhinella acutirostris and four undescribed species (see also ML topology in supplementary data 4.5): Rhinella sp. 10 from Napo (Ecuador), Rhinella sp. 11 from Amazonas (Brazil), Rhinella sp. 12 from Pando (Bolivia) and Madre de Dios (Peru), and Rhinella sp. 13 from some localities of Colombia (Boyacá and Caquetá), Peru (Loreto), and Venezuela (Miranda). This latter new species was previously recorded from Colombia as $R$. sternosignata (M.R., personal obs.; see comments for $R$. sternosignata).

The nonmonophyletic Rhinella castaneotica and $R$. proboscidea compose a well-supported clade (JGC and JAF $=96 \%$ ). The internal relationships among the included specimens are poorly resolved and the UPDs are $0.19 \%-2.75 \%$. Within this clade, we could not obtain samples from the type locality of $R$. castaneotica ("near Cachoeira Jaruá, Rio Xingu, Pará, Brazil"), but we included sequences from a relatively close locality $(300 \mathrm{~km}$ airline distance) that could represent $R$. castaneotica s.s. (see Fouquet et al., 2012a: fig. S6). We also included sequences of specimens from Manaus (Amazonas, Brazil) and Floresta (Roraima, Brazil) that could be morphologically assigned to $R$. proboscidea. However, the imprecise type locality of this species is "flumen Solimoens (= Rio Solimões)," which comprises the Brazilian section of the Amazon River between the triple border of Brazil-Colombia-Peru and the city of Manaus and has an extension of approximately $1700 \mathrm{~km}$ (Vanzolini, 1981). Although we consider that phylogenetic evidence, UPDs (see table 11), and absence of distinctive adult and larval differential characters (see comparisons provided by Caldwell, 1991, and Menin et al., 2006) support their conspecific- ity, we continue recognizing both taxa pending a thorough revision, including additional samples and detailed comparison with the type material.

The sister taxon of the clade including the problematic Rhinella castaneotica and $R$. proboscidea, is well supported (JGC $=97 \%$, JAF $=98 \%$ ) and includes two successively diverging species (R. lescurei and R. hoogmoedi), and two subclades. One of these is well supported (JGC and JAF $=99 \%$ ) and composed of $R$. paraguayensis, $R$. scitula, R. stanlaii, and two undescribed species: Rhinella sp. 14 from SE Peru ("Bufo sp. 6" sensu Vélez-Rodríguez, 2004b, and "Bufo cf. margaritifer 5" sensu Pramuk, 2006), and Rhinella sp. 15 from La Paz, Bolivia (which corresponds to Bufo sp. 1 of Lötters and Köhler, 2000). The included specimen of $R$. paraguayensis was recovered in a polytomy with the specimens of R. scitula; the UPDs among these specimens are low $(0.13 \%-0.3 \%$ in the complete the $16 \mathrm{~S}$ rRNA gene). These parapatric species were differentiated mainly by adult size, crest development, and skin texture. All these characters have been demonstrated to be subject to variation due to specific environmental conditions throughout the distribution of some bufonids (see Kutrup et al., 2006; Bandeira et al., 2016). Thus, we consider Rhinella paraguayensis Ávila et al., 2010, to be a junior synonym of $R$. scitula (Caramaschi and Niemeyer, 2003).

The other subclade is also well supported (JGC and JAF $=98 \%$ ) and includes Rhinella margaritifera s.s. and a poorly supported clade (JGC $<25 \%$, JAF $<50 \%$ ) composed of $R$. dapsilis, $R$. cf. dapsilis, $R$. gildae, and several divergent lineages of $R$. margaritifera s.l. (e.g., the lineages called Rhinella sp. A and Rhinella sp. B by Fouquet et al., 2007c). The clade includes specimens that vary in the degree of development of bony protrusions and cranial crests, dorsal coloration, occurrence of a developed proboscis, and dorsal skin texture (Myers and Carvalho, 1945; Dixon, 1976; Rodríguez and Duellman, 1994; Vaz-Silva et al., 2015; M.O.P., personal obs.). The UPDs within this clade are 0\%-2.79\% (mean UPD = $1.29 \%$, see table 11 ). Thus, the absence of 
unequivocal morphological differences and very low genetic distances indicate that Rhinella gildae Vaz-Silva et al., 2015, is a junior synonym of $R$. dapsilis (Myers and Carvalho, 1945).

We could not obtain tissue samples of Rhinella cristinae to test its relationships. However, this species can be assigned to this group on the basis of the occurrence of the only putative phenotypic synapomorphy of the group (acuminate anterior margins of nasals; char. 6.1) and a combination of characters typical of this group: (1) preorbital crest weak (char. 0.1), (2) medial ramus of the pterygoid fused and extending medially along approximately half the length of the parasphenoid ala (char. 21.2), (3) posterior lobe of the anterolateral process of hyoid absent (char. 25.0), (4) inguinal fat bodies absent (char. 51.0), and (5) tarsal fold absent (char. 65.0).

\section{Hybridization, Deep Mitochondrial} Divergence, AND "GHost Introgression"

\section{IN RHINELLA}

Reports on natural and artificial hybridization are well known in many bufonids including multiple species of Rhinella (e.g., Blair, 1972; Green, 1996; Gergus et al., 1999; Malmos et al., 2001; Masta et al., 2002; Baldo and Basso, 2004; Yamazaki et al., 2008; Goebel et al., 2009; Fontenot et al., 2011; Correa et al., 2012, 2013; Pereyra et al., 2016a; Betto-Colliard et al., 2018). Explosive breeding events with intense male competition for mates and passive female choice (i.e., scramble competition; see Wells, 2007; Pereyra et al., 2016b) is common in many species of several genera of Bufonidae, and premating isolating mechanisms seem to be insufficient to avoid interspecific amplexus in these species (see Blair, 1958; Guerra et al., 2011). Malone and Fontenot (2008) also demonstrated that bufonids require a substantial genetic divergence to achieve postzygotic reproductive isolation. Under this scenario, the common occurrence of hybridization in this family is not surprising.

A particular situation of natural hybridization could happen in "Rhinella pombali" (Thomé et al., 2010, 2012), where all individuals of this taxon are considered hybrids between $R$. crucifer and $R$. ornata (Thomé et al., 2010, 2012) and our results are in agreement with this idea. The two included specimens of " $R$. pombali" in preliminary analyses were not recovered as monophyletic in the nuclear analysis and each of them has a unique mitochondrion (one from $R$. crucifer and the other from $R$. ornata). Moreover, available evidence is insufficient to test whether " $R$. pombali" could represent a species of hybrid origin (see Avise, 2008; Darras and Aron, 2015, Lavanchy and Schwander, 2019) and more detailed studies are necessary to explore this possibility.

Although we deliberately excluded the hybrid specimens from our analyses, the impact of natural hybridization in Bufonidae could be currently underestimated due to the difficulties in recognizing hybrids and/or past hybridization events. Introgressive hybridization (both nuclear and mitochondrial) could have an impact on bufonid evolution allowing a faster accumulation of genetic novelties than through mutation alone. The incorporation of additional genetic diversity could impact the acquisition of adaptive phenotypic traits and have a significant role in speciation as is common in diverse taxonomic groups (for reviews see Baack and Rieseberg, 2007; Schwenk et al., 2008; Toews and Brelsford, 2012; Abbott et al., 2016; Gopalakrishnan et al., 2018; Hill, 2019; Servedio and Hermisson, 2019).

Mitochondrial introgressions are more commonly reported than are nuclear introgressions and can be evidenced by genetic populational studies or by the discordance between phylogenetic trees inferred from separate analyses of both genomes (Toews and Brelsford, 2012; Bonnet et al., 2017). Within Rhinella, putative events of mitochondrial introgression were documented for R. marina (Sequeira et al., 2011; but also see Vallinoto et al., 2017 and Bessa-Silva et al., 2020), $R$. bernardoi (Pereyra et al., 2016a), and R. horribilis s.l. (present study), and they occur without noticeable evidence of nuclear introgression, as was also reported in other vertebrates (Alves et al., 2006; Chen et al., 2009; Schwarzer et al., 2012). 
TABLE 11

Percentage of uncorrected p-distances between $16 \mathrm{~S}$ sequences among species of the Rhinella margaritifera Group Values reported are mean (range).

\begin{tabular}{|c|c|c|c|c|c|c|c|c|c|c|c|c|c|}
\hline & & 1 & 2 & 3 & 4 & 5 & 6 & 7 & 8 & 9 & 10 & 11 & 12 \\
\hline 1 & Rhinella sp. $5(\mathrm{~N}=2)$ & 0.00 & & & & & & & & & & & \\
\hline 2 & $\begin{array}{l}\text { R. ocellata } \\
(\mathrm{N}=4)\end{array}$ & $\begin{array}{c}7.58 \\
(6.71-8.99) \\
\end{array}$ & $\begin{array}{c}0.49 \\
(0.00-0.96)\end{array}$ & & & & & & & & & & \\
\hline 3 & $\begin{array}{l}\text { R. iserni } \\
(\mathrm{N}=4)\end{array}$ & $\begin{array}{c}5.34 \\
(5.29-5.39) \\
\end{array}$ & $\begin{array}{c}4.63 \\
(4.23-5.27) \\
\end{array}$ & $\begin{array}{c}0.00 \\
(0.00)\end{array}$ & & & & & & & & & \\
\hline 4 & $\begin{array}{l}\text { R. magnussoni } \\
(\mathrm{N}=1)\end{array}$ & $\begin{array}{c}6.12 \\
(6.08-6.16) \\
\end{array}$ & $\begin{array}{c}6.11 \\
(5.75-6.83) \\
\end{array}$ & $\begin{array}{c}3.24 \\
(3.24) \\
\end{array}$ & - & & & & & & & & \\
\hline 5 & $\begin{array}{l}\text { Rhinella sp. } 6 \\
(\mathrm{~N}=1)\end{array}$ & $\begin{array}{c}5.34 \\
(5.29-5.39) \\
\end{array}$ & $\begin{array}{c}4.95 \\
(4.62-5.58) \\
\end{array}$ & $\begin{array}{c}3.44 \\
(3.44) \\
\end{array}$ & 2.10 & - & & & & & & & \\
\hline 6 & $\begin{array}{l}\text { Rhinella sp. } 7 \\
(\mathrm{~N}=1)\end{array}$ & $\begin{array}{c}6.95 \\
(6.91-6.98) \\
\end{array}$ & $\begin{array}{c}7.97 \\
(7.24-9.86) \\
\end{array}$ & $\begin{array}{c}4.58 \\
(4.58) \\
\end{array}$ & 3.50 & 3.97 & - & & & & & & \\
\hline 7 & $\begin{array}{l}\text { Rhinella sp. } 8 \\
(\mathrm{~N}=1)\end{array}$ & $\begin{array}{c}13.45 \\
(13.42- \\
13.49)\end{array}$ & $\begin{array}{c}15.55 \\
(15.04- \\
16.39)\end{array}$ & $\begin{array}{c}12.25 \\
(12,25)\end{array}$ & 11.67 & 11.65 & 9.66 & - & & & & & \\
\hline 8 & $\begin{array}{l}\text { Rhinella sp. } 9 \\
(\mathrm{~N}=3)\end{array}$ & $\begin{array}{c}5.87 \\
(5.69-6.01) \\
\end{array}$ & $\begin{array}{c}6.88 \\
(6.40-8.04) \\
\end{array}$ & $\begin{array}{c}4.17 \\
(3.85-4.39) \\
\end{array}$ & $\begin{array}{c}3.58 \\
(3.25-3.82) \\
\end{array}$ & $\begin{array}{c}3.33 \\
(3.26-3.44) \\
\end{array}$ & $\begin{array}{c}2.77 \\
(2.61-2.90) \\
\end{array}$ & $\begin{array}{c}9.93(9.63- \\
10.29) \\
\end{array}$ & $\begin{array}{c}0.66 \\
(0.58-0.80) \\
\end{array}$ & & & & \\
\hline 9 & $\begin{array}{l}\text { R. sclerocephala } \\
(\mathrm{N}=1)\end{array}$ & $\begin{array}{c}7.81 \\
(7.80-7.82) \\
\end{array}$ & $\begin{array}{c}8.64 \\
(7.99-9.84) \\
\end{array}$ & $\begin{array}{r}5.34 \\
(5.34) \\
\end{array}$ & 5.13 & 6.46 & 6.94 & 14.95 & $\begin{array}{c}7.20 \\
(6.85-7.51) \\
\end{array}$ & - & & & \\
\hline 10 & $\begin{array}{l}\text { "R. alata" } \\
(\mathrm{N}=5)\end{array}$ & $\begin{array}{c}5.75 \\
(6.68- \\
6.94) \\
\end{array}$ & $\begin{array}{c}6.99 \\
(8.42-)\end{array}$ & $\begin{array}{c}3.98 \\
(3.68- \\
4.21) \\
\end{array}$ & $\begin{array}{c}4.48 \\
(4.20- \\
4.78) \\
\end{array}$ & $\begin{array}{c}4.90 \\
(4.63- \\
5.15) \\
\end{array}$ & $\begin{array}{c}6.11 \\
(5.60- \\
6.42) \\
\end{array}$ & $\begin{array}{c}14.01 \\
(13.86- \\
14.19) \\
\end{array}$ & $\begin{array}{c}5.91 \\
(5.31- \\
6.30) \\
\end{array}$ & $\begin{array}{c}1.41 \\
(1.28- \\
1.50) \\
\end{array}$ & $\begin{array}{c}0.96 \\
(0.57- \\
1.35) \\
\end{array}$ & & \\
\hline 11 & $\begin{array}{l}\text { Rhinella sp. } 10 \\
(\mathrm{~N}=1)\end{array}$ & $\begin{array}{c}7.11 \\
(7.07-7.14) \\
\end{array}$ & $\begin{array}{c}5.76 \\
(5.24-6.78) \\
\end{array}$ & $\begin{array}{c}4.45 \\
(4.45) \\
\end{array}$ & 5.21 & 4.45 & 6.90 & 14.69 & $\begin{array}{c}6.09 \\
(6.01-6.17) \\
\end{array}$ & 3.82 & $\begin{array}{c}3.21 \\
(2.90-3.67) \\
\end{array}$ & - & \\
\hline 12 & $\begin{array}{l}\text { Rhinella sp. } 11 \\
(\mathrm{~N}=1)\end{array}$ & $\begin{array}{c}7.41 \\
(7.28-7.53) \\
\end{array}$ & $\begin{array}{c}6.12 \\
(5.57-7.03) \\
\end{array}$ & $\begin{array}{c}4.78 \\
(4.78) \\
\end{array}$ & 5.93 & 4.97 & 7.24 & 15.17 & $\begin{array}{c}6.40 \\
(6.30-6.49) \\
\end{array}$ & 4.46 & $\begin{array}{c}3.63 \\
(3.28-4.00) \\
\end{array}$ & 0.78 & - \\
\hline 13 & $\begin{array}{l}\text { Rhinella sp. } 12 \\
(\mathrm{~N}=3)\end{array}$ & $\begin{array}{c}7.90 \\
(7.07- \\
9.31) \\
\end{array}$ & $\begin{array}{c}6.14 \\
(5.19- \\
7.08) \\
\end{array}$ & $\begin{array}{c}4.95 \\
(4.40- \\
5.83) \\
\end{array}$ & $\begin{array}{c}6.56 \\
(5.92- \\
7.63) \\
\end{array}$ & $\begin{array}{c}5.41 \\
(4.79- \\
6.43) \\
\end{array}$ & $\begin{array}{c}8.40 \\
(7.26- \\
10.42) \\
\end{array}$ & $\begin{array}{c}15.87 \\
(15.17- \\
17.06) \\
\end{array}$ & $\begin{array}{c}7.00 \\
(6.40- \\
8.09) \\
\end{array}$ & $\begin{array}{c}4.64 \\
(4.25- \\
5.19) \\
\end{array}$ & $\begin{array}{c}3.74 \\
(2.90- \\
5.14) \\
\end{array}$ & $\begin{array}{c}1.36 \\
(1.16- \\
1.55) \\
\end{array}$ & $\begin{array}{c}1.68 \\
(1.53- \\
1.79) \\
\end{array}$ \\
\hline 14 & $\begin{array}{l}\text { R. acutirostris } \\
(\mathrm{N}=5)\end{array}$ & $\begin{array}{c}7.31 \\
(6.90- \\
7.73) \\
\end{array}$ & $\begin{array}{c}7.09 \\
(5.97- \\
8.46) \\
\end{array}$ & $\begin{array}{c}4.99 \\
(4.62- \\
5.37) \\
\end{array}$ & $\begin{array}{c}6.20 \\
(5.76- \\
6.51) \\
\end{array}$ & $\begin{array}{c}5.83 \\
(5.56- \\
6.14) \\
\end{array}$ & $\begin{array}{c}8.00 \\
(7.58- \\
8.42) \\
\end{array}$ & $\begin{array}{c}15.43 \\
(15.02- \\
15.58) \\
\end{array}$ & $\begin{array}{c}7.04 \\
(6.62- \\
7.35) \\
\end{array}$ & $\begin{array}{c}4.28 \\
(4.11- \\
4.52) \\
\end{array}$ & $\begin{array}{c}3.72 \\
(3.26-)\end{array}$ & $\begin{array}{c}2.12 \\
(1.93- \\
2.31) \\
\end{array}$ & $\begin{array}{c}2.88 \\
(2.68- \\
3.07) \\
\end{array}$ \\
\hline 15 & $\begin{array}{l}\text { Rhinella sp. } 13 \\
(\mathrm{~N}=6)\end{array}$ & $\begin{array}{c}6.81 \\
(6.69- \\
7.09) \\
\end{array}$ & $\begin{array}{c}6.55 \\
(5.59- \\
7.92) \\
\end{array}$ & $\begin{array}{c}5.45 \\
(5.19- \\
5.83) \\
\end{array}$ & $\begin{array}{c}6.39 \\
(6.12- \\
6.76) \\
\end{array}$ & $\begin{array}{c}5.51 \\
(5.18- \\
5.82) \\
\end{array}$ & $\begin{array}{c}8.07 \\
(7.78- \\
8.57) \\
\end{array}$ & $\begin{array}{c}15.58 \\
(15.38- \\
15.82) \\
\end{array}$ & $\begin{array}{c}7.29 \\
(6.98- \\
7.72) \\
\end{array}$ & $\begin{array}{c}4.77 \\
(4.55- \\
5.20) \\
\end{array}$ & $\begin{array}{c}4.13 \\
(3.63- \\
4.85) \\
\end{array}$ & $\begin{array}{c}2.83 \\
(2.51- \\
3.09) \\
\end{array}$ & $\begin{array}{c}3.58 \\
(3.26- \\
3.88) \\
\end{array}$ \\
\hline 16 & $\begin{array}{l}\text { "R. castaneotica" } \\
(\mathrm{N}=4)\end{array}$ & $\begin{array}{c}6.46 \\
(5.69- \\
7.87) \\
\end{array}$ & $\begin{array}{c}5.81 \\
(4.79- \\
7.21) \\
\end{array}$ & $\begin{array}{c}3.47 \\
(2.87- \\
4.86) \\
\end{array}$ & $\begin{array}{c}4.59 \\
(4.01- \\
5.92) \\
\end{array}$ & $\begin{array}{c}4.33 \\
(3.83- \\
5.45) \\
\end{array}$ & $\begin{array}{c}5.66 \\
(4.64- \\
7.68) \\
\end{array}$ & $\begin{array}{c}13.42 \\
(12.65- \\
14.94) \\
\end{array}$ & $\begin{array}{c}4.80 \\
(3.86- \\
6.12) \\
\end{array}$ & $\begin{array}{c}4.61 \\
(4.08- \\
5.60) \\
\end{array}$ & $\begin{array}{c}3.47 \\
(2.70- \\
4.97) \\
\end{array}$ & $\begin{array}{c}3.69 \\
(3.28- \\
4.35) \\
\end{array}$ & $\begin{array}{c}4.02 \\
(3.65- \\
4.60) \\
\end{array}$ \\
\hline 17 & $\begin{array}{l}\text { R. proboscidea } \\
(\mathrm{N}=3)\end{array}$ & $\begin{array}{c}6.94 \\
(6.08- \\
8.61) \\
\end{array}$ & $\begin{array}{c}6.53 \\
(5.18- \\
8.21) \\
\end{array}$ & $\begin{array}{c}4.11 \\
(3.25- \\
5.33) \\
\end{array}$ & $\begin{array}{c}4.98 \\
(4.21- \\
6.38) \\
\end{array}$ & $\begin{array}{c}4.79 \\
(3.83- \\
6.20) \\
\end{array}$ & $\begin{array}{c}6.36 \\
(4.81- \\
7.93) \\
\end{array}$ & $\begin{array}{c}13.91 \\
(13.25- \\
14.90) \\
\end{array}$ & $\begin{array}{c}5.64 \\
(4.47- \\
7.12) \\
\end{array}$ & $\begin{array}{c}4.69 \\
(4.06- \\
5.53) \\
\end{array}$ & $\begin{array}{c}3.79 \\
(2.70- \\
4.91) \\
\end{array}$ & $\begin{array}{c}4.05 \\
(3.09- \\
5.11) \\
\end{array}$ & $\begin{array}{c}4.46 \\
(3.45- \\
5.37) \\
\end{array}$ \\
\hline 18 & $\begin{array}{l}\text { R. lescurei } \\
(\mathrm{N}=3)\end{array}$ & $\begin{array}{c}7.57 \\
(6.47- \\
8.41) \\
\end{array}$ & $\begin{array}{c}6.05 \\
(4.80- \\
6.91) \\
\end{array}$ & $\begin{array}{c}5.79 \\
(4.77- \\
6.46) \\
\end{array}$ & $\begin{array}{c}6.03 \\
(5.15- \\
6.50) \\
\end{array}$ & $\begin{array}{c}5.44 \\
(4.59- \\
6.02) \\
\end{array}$ & $\begin{array}{c}8.67 \\
(6.77- \\
9.93) \\
\end{array}$ & $\begin{array}{c}14.50 \\
(13.58- \\
15.17) \\
\end{array}$ & $\begin{array}{c}6.51 \\
(5.61- \\
7.41) \\
\end{array}$ & $\begin{array}{c}6.53 \\
(5.74- \\
6.95) \\
\end{array}$ & $\begin{array}{c}5.26 \\
(4.39- \\
5.76) \\
\end{array}$ & $\begin{array}{c}5.72 \\
(4.82- \\
6.19) \\
\end{array}$ & $\begin{array}{c}5.99 \\
(5.16- \\
6.44) \\
\end{array}$ \\
\hline 19 & $\begin{array}{l}\text { R. hoogmoedi } \\
(\mathrm{N}=4)\end{array}$ & $\begin{array}{c}7.03 \\
(6.48- \\
8.33) \\
\end{array}$ & $\begin{array}{c}6.33 \\
(5.38- \\
7.40) \\
\end{array}$ & $\begin{array}{c}4.78 \\
(4.40- \\
5.57) \\
\end{array}$ & $\begin{array}{c}4.93 \\
(4.59- \\
5.61) \\
\end{array}$ & $\begin{array}{c}4.81 \\
(4.40- \\
5.67) \\
\end{array}$ & $\begin{array}{c}7.10 \\
(6.40- \\
8.60) \\
\end{array}$ & $\begin{array}{c}13.99 \\
(13.65- \\
14.63) \\
\end{array}$ & $\begin{array}{c}6.25 \\
(5.50- \\
7.39) \\
\end{array}$ & $\begin{array}{c}5.34 \\
(4.95- \\
6.09) \\
\end{array}$ & $\begin{array}{c}4.24 \\
(3.82- \\
5.17) \\
\end{array}$ & $\begin{array}{c}4.98 \\
(4.45- \\
5.87) \\
\end{array}$ & $\begin{array}{c}5.26 \\
(4.79- \\
6.12) \\
\end{array}$ \\
\hline 20 & $\begin{array}{l}\text { Rhinella sp. } 14 \\
(\mathrm{~N}=7)\end{array}$ & $\begin{array}{c}6.32 \\
(6.09- \\
6.57) \\
\end{array}$ & $\begin{array}{c}5.14 \\
(4.22- \\
6.52) \\
\end{array}$ & $\begin{array}{c}4.06 \\
(3.83- \\
4.40) \\
\end{array}$ & $\begin{array}{c}4.46 \\
(4.21- \\
4.83) \\
\end{array}$ & $\begin{array}{c}4.11 \\
(4.01- \\
4.27) \\
\end{array}$ & $\begin{array}{c}5.90 \\
(5.72- \\
6.25) \\
\end{array}$ & $\begin{array}{c}13.35 \\
(13.03- \\
13.42) \\
\end{array}$ & $\begin{array}{c}5.71 \\
(5.10- \\
6.18) \\
\end{array}$ & $\begin{array}{c}4.97 \\
(4.70- \\
5.19) \\
\end{array}$ & $\begin{array}{c}3.74 \\
(3.43- \\
4.25) \\
\end{array}$ & $\begin{array}{c}3.83 \\
(3.47- \\
4.06) \\
\end{array}$ & $\begin{array}{c}4.18 \\
(3.81- \\
4.45) \\
\end{array}$ \\
\hline 21 & $\begin{array}{l}\text { Rhinella sp. } 15 \\
(\mathrm{~N}=1)\end{array}$ & $\begin{array}{c}5.93 \\
(5.89-5.97) \\
\end{array}$ & $\begin{array}{c}5.86 \\
(5.18-6.78) \\
\end{array}$ & $\begin{array}{c}3.82 \\
(3.82) \\
\end{array}$ & 4.39 & 4.02 & 5.95 & 13.04 & $\begin{array}{c}5.35 \\
(5.09-5.53) \\
\end{array}$ & 4.71 & $\begin{array}{c}3.48 \\
(3.44-3.63) \\
\end{array}$ & 3.47 & 3.82 \\
\hline 22 & $\begin{array}{l}\text { R. scitula } \\
(\mathrm{N}=2)\end{array}$ & $\begin{array}{c}6.12 \\
(6.09- \\
6.16) \\
\end{array}$ & $\begin{array}{c}6.48 \\
(5.76- \\
7.55) \\
\end{array}$ & $\begin{array}{c}4.01 \\
(4.01)\end{array}$ & $\begin{array}{c}4.58 \\
(4.58)\end{array}$ & $\begin{array}{c}4.21 \\
(4.21)\end{array}$ & $\begin{array}{c}6.16 \\
(6.16)\end{array}$ & $\begin{array}{c}13.23 \\
(13.23)\end{array}$ & $\begin{array}{c}5.54 \\
(5.29- \\
5.72) \\
\end{array}$ & $\begin{array}{c}4.71 \\
(4.71)\end{array}$ & $\begin{array}{c}3.56 \\
(3.44- \\
3.63) \\
\end{array}$ & $\begin{array}{c}3.86 \\
(3.86)\end{array}$ & $\begin{array}{c}4.20 \\
(4.20)\end{array}$ \\
\hline 23 & $\begin{array}{l}\text { R. stanlaii } \\
(\mathrm{N}=4)\end{array}$ & $\begin{array}{c}6.01 \\
(5.71- \\
6.16) \\
\end{array}$ & $\begin{array}{c}5.98 \\
(5.19- \\
6.99) \\
\end{array}$ & $\begin{array}{c}3.88 \\
(3.64- \\
4.01) \\
\end{array}$ & $\begin{array}{c}4.50 \\
(4.40- \\
4.58) \\
\end{array}$ & $\begin{array}{c}4.17 \\
(4.08- \\
4.21) \\
\end{array}$ & $\begin{array}{c}5.98 \\
(5.17- \\
6.14) \\
\end{array}$ & $\begin{array}{c}12.81 \\
(12.71- \\
12.84) \\
\end{array}$ & $\begin{array}{c}5.41 \\
(4.88- \\
5.72) \\
\end{array}$ & $\begin{array}{c}4.64 \\
(4.50- \\
4.69) \\
\end{array}$ & $\begin{array}{c}3.52 \\
(3.29- \\
3.63) \\
\end{array}$ & $\begin{array}{c}3.43 \\
(3.33- \\
3.47) \\
\end{array}$ & $\begin{array}{c}3.78 \\
(3.68- \\
3.82) \\
\end{array}$ \\
\hline 24 & $\begin{array}{l}\text { R. margaritifera } \\
(\mathrm{N}=3)\end{array}$ & $\begin{array}{c}5.99 \\
(4.91- \\
6.52) \\
\end{array}$ & $\begin{array}{c}6.22 \\
(4.99- \\
7.13) \\
\end{array}$ & $\begin{array}{c}4.63 \\
(3.82- \\
5.04) \\
\end{array}$ & $\begin{array}{c}4.01 \\
(3.44- \\
4.30) \\
\end{array}$ & $\begin{array}{c}4.47 \\
(3.63- \\
4.89) \\
\end{array}$ & $\begin{array}{c}5.74 \\
(4.63- \\
6.30) \\
\end{array}$ & $\begin{array}{c}12.77 \\
(12.25- \\
13.03) \\
\end{array}$ & $\begin{array}{c}5.14 \\
(4.29- \\
5.83) \\
\end{array}$ & $\begin{array}{c}5.70 \\
(4.95- \\
6.07) \\
\end{array}$ & $\begin{array}{c}4.45 \\
(3.63- \\
4.91) \\
\end{array}$ & $\begin{array}{c}5.85 \\
(4.82- \\
6.37) \\
\end{array}$ & $\begin{array}{c}6.13 \\
(5.16- \\
6.62)\end{array}$ \\
\hline 25 & $\begin{array}{l}\text { R. dapsilis } \\
(\mathrm{N}=15)\end{array}$ & $\begin{array}{c}6.64 \\
(5.69- \\
8.59)\end{array}$ & $\begin{array}{c}6.04 \\
(4.80- \\
7.42)\end{array}$ & $\begin{array}{c}4.10 \\
(3.63- \\
5.06) \\
\end{array}$ & $\begin{array}{c}4.74 \\
(4.01- \\
5.88) \\
\end{array}$ & $\begin{array}{c}4.91 \\
(4.09- \\
6.20) \\
\end{array}$ & $\begin{array}{c}5.99 \\
(5.10- \\
7.68) \\
\end{array}$ & $\begin{array}{c}13.40 \\
(12.18- \\
14.35)\end{array}$ & $\begin{array}{c}5.77 \\
(4.90- \\
7.12) \\
\end{array}$ & $\begin{array}{c}5.10 \\
(4.30- \\
6.40) \\
\end{array}$ & $\begin{array}{c}3.93 \\
(3.05- \\
5.19) \\
\end{array}$ & $\begin{array}{c}4.92 \\
(4.07- \\
6.40) \\
\end{array}$ & $\begin{array}{c}5.21 \\
(4.27- \\
6.65) \\
\end{array}$ \\
\hline
\end{tabular}


TABLE 11 continued

$\begin{array}{lllllllllllll}13 & 14 & 15 & 16 & 17 & 18 & 19 & 20 & 21 & 22 & 23 & 24 & 24\end{array}$

\begin{tabular}{|c|c|c|c|c|c|c|c|c|c|c|c|c|c|c|}
\hline 13 & $\begin{array}{l}\text { Rhinella sp. } 12 \\
(\mathrm{~N}=3)\end{array}$ & $\begin{array}{c}0.06 \\
(0.00- \\
0.19) \\
\end{array}$ & & & & & & & & & & & & \\
\hline 14 & $\begin{array}{l}\text { R. acutirostris } \\
(\mathrm{N}=5)\end{array}$ & $\begin{array}{c}2.86 \\
(2.29- \\
3.66) \\
\end{array}$ & $\begin{array}{c}0.69 \\
(0.19- \\
1.15) \\
\end{array}$ & & & & & & & & & & & \\
\hline 15 & $\begin{array}{l}\text { Rhinella sp. } 13 \\
(\mathrm{~N}=6)\end{array}$ & $\begin{array}{c}3.63 \\
(2.86- \\
4.70) \\
\end{array}$ & $\begin{array}{c}1.67 \\
(1.34- \\
2.13) \\
\end{array}$ & $\begin{array}{c}0.49 \\
(0.00- \\
0.77)\end{array}$ & & & & & & & & & & \\
\hline 16 & $\begin{array}{l}\text { " } R, \text { castaneotica" } \\
(\mathrm{N}=4)\end{array}$ & $\begin{array}{c}4.40 \\
(3.64- \\
5.34) \\
\end{array}$ & $\begin{array}{c}4.85 \\
(4.23- \\
5.79) \\
\end{array}$ & $\begin{array}{c}5.09 \\
(4.23- \\
6.58) \\
\end{array}$ & $\begin{array}{c}1.19 \\
(0.19- \\
2.28) \\
\end{array}$ & & & & & & & & & \\
\hline 17 & $\begin{array}{l}\text { R. proboscidea } \\
(\mathrm{N}=3)\end{array}$ & $\begin{array}{c}4.91 \\
(3.44- \\
5.90)\end{array}$ & $\begin{array}{c}4.99 \\
(4.02- \\
6.26)\end{array}$ & $\begin{array}{c}5.20 \\
(4.03- \\
6.80)\end{array}$ & $\begin{array}{c}1.82 \\
(0.76- \\
2.83)\end{array}$ & $\begin{array}{c}1.31 \\
(0.98- \\
1.78)\end{array}$ & & & & & & & & \\
\hline 18 & $\begin{array}{l}\text { R. lescurei } \\
(\mathrm{N}=3)\end{array}$ & $\begin{array}{c}5.99 \\
(4.77- \\
6.46)\end{array}$ & $\begin{array}{c}6.50 \\
(5.35- \\
7.09)\end{array}$ & $\begin{array}{c}6.09 \\
(4.96- \\
7.10)\end{array}$ & $\begin{array}{c}4.16 \\
(3.24- \\
4.90)\end{array}$ & $\begin{array}{c}4.80 \\
(3.82- \\
5.20)\end{array}$ & $\begin{array}{c}0.85 \\
(0.00- \\
1.28)\end{array}$ & & & & & & & \\
\hline 19 & $\begin{array}{l}\text { R. hoogmoedi } \\
(\mathrm{N}=4)\end{array}$ & $\begin{array}{c}5.80 \\
(4.79- \\
6.68)\end{array}$ & $\begin{array}{c}5.56 \\
(4.97- \\
6.77)\end{array}$ & $\begin{array}{c}5.31 \\
(4.60- \\
6.78)\end{array}$ & $\begin{array}{c}3.30 \\
(2.57- \\
4.43)\end{array}$ & $\begin{array}{c}3.92 \\
(3.25- \\
4.38)\end{array}$ & $\begin{array}{c}2.90 \\
(2.49- \\
3.17)\end{array}$ & $\begin{array}{c}0.46 \\
(0.00- \\
0.79)\end{array}$ & & & & & & \\
\hline 20 & $\begin{array}{l}\text { Rhinella sp. } 14 \\
(\mathrm{~N}=7)\end{array}$ & $\begin{array}{c}4.90 \\
(4.20- \\
6.15)\end{array}$ & $\begin{array}{c}4.46 \\
(4.02- \\
5.04)\end{array}$ & $\begin{array}{c}4.12 \\
(3.63- \\
4.46)\end{array}$ & $\begin{array}{c}3.51 \\
(2.29- \\
4.91)\end{array}$ & $\begin{array}{c}3.77 \\
(2.87- \\
4.86)\end{array}$ & $\begin{array}{c}3.48 \\
(2.48- \\
4.14)\end{array}$ & $\begin{array}{c}2.39 \\
(1.53- \\
2.82)\end{array}$ & $\begin{array}{c}0.57 \\
(0.00- \\
0.97)\end{array}$ & & & & & \\
\hline 21 & $\begin{array}{l}\text { Rhinella sp. } 15 \\
(\mathrm{~N}=1)\end{array}$ & $\begin{array}{c}4.76 \\
(4.20- \\
5.65) \\
\end{array}$ & $\begin{array}{c}4.55 \\
(4.40- \\
4.78)\end{array}$ & $\begin{array}{c}4.66 \\
(4.40- \\
5.03) \\
\end{array}$ & $\begin{array}{c}2.91 \\
(2.29- \\
4.39) \\
\end{array}$ & $\begin{array}{c}3.28 \\
(2.86- \\
3.82) \\
\end{array}$ & $\begin{array}{c}3.83 \\
(3.25- \\
4.14)\end{array}$ & $\begin{array}{c}2.48 \\
(2.29- \\
2.78) \\
\end{array}$ & $\begin{array}{c}1.50 \\
(1.15- \\
1.74)\end{array}$ & - & & & & \\
\hline 22 & $\begin{array}{l}R, \text { scitula } \\
(\mathrm{N}=2)\end{array}$ & $\begin{array}{c}4.75 \\
(4.20- \\
5.65)\end{array}$ & $\begin{array}{c}4.55 \\
(4.40- \\
4.78)\end{array}$ & $\begin{array}{c}4.66 \\
(4.40- \\
5.02)\end{array}$ & $\begin{array}{c}3.01 \\
(2.48- \\
4.40)\end{array}$ & $\begin{array}{c}3.22 \\
(2.87- \\
3.84)\end{array}$ & $\begin{array}{c}3.61 \\
(3.06- \\
3.90)\end{array}$ & $\begin{array}{c}2.28 \\
(2.10- \\
2.54)\end{array}$ & $\begin{array}{c}2.24 \\
(1.72- \\
2.51)\end{array}$ & $\begin{array}{c}1.15 \\
(1.15)\end{array}$ & $\begin{array}{c}0.00 \\
(0.00)\end{array}$ & & & \\
\hline 23 & $\begin{array}{l}\text { R. stanlaii } \\
(\mathrm{N}=4)\end{array}$ & $\begin{array}{c}4.71 \\
(4.07- \\
5.62)\end{array}$ & $\begin{array}{c}4.53 \\
(4.28- \\
4.79)\end{array}$ & $\begin{array}{c}4.62 \\
(4.28- \\
5.04)\end{array}$ & $\begin{array}{c}3.25 \\
(2.71- \\
4.37)\end{array}$ & $\begin{array}{c}3.48 \\
(2.72- \\
4.33)\end{array}$ & $\begin{array}{c}3.82 \\
(3.05- \\
4.36)\end{array}$ & $\begin{array}{c}2.65 \\
(2.33- \\
3.04)\end{array}$ & $\begin{array}{c}1.79 \\
(1.55- \\
2.33)\end{array}$ & $\begin{array}{c}1.01 \\
(0.78- \\
1.34)\end{array}$ & $\begin{array}{c}0.96 \\
(0.78- \\
1.15)\end{array}$ & $\begin{array}{c}0.42 \\
(0.00- \\
0.77)\end{array}$ & & \\
\hline 24 & $\begin{array}{l}\text { R. margaritifera } \\
(\mathrm{N}=3)\end{array}$ & $\begin{array}{c}6.54 \\
(5.15- \\
6.94)\end{array}$ & $\begin{array}{r}6.63 \\
(5.36- \\
7.27)\end{array}$ & $\begin{array}{c}6.37 \\
(4.97- \\
7.27)\end{array}$ & $\begin{array}{c}4.00 \\
(2.86- \\
5.20)\end{array}$ & $\begin{array}{c}4.63 \\
(3.44- \\
5.15)\end{array}$ & $\begin{array}{c}4.07 \\
(3.25- \\
4.27)\end{array}$ & $\begin{array}{c}3.04 \\
(2.48- \\
3.33)\end{array}$ & $\begin{array}{c}2.83 \\
(2.10- \\
3.32)\end{array}$ & $\begin{array}{c}3.49 \\
(2.86- \\
3.80)\end{array}$ & $\begin{array}{c}3.72 \\
(3.05- \\
4.05)\end{array}$ & $\begin{array}{c}3.54 \\
(2.68- \\
4.02)\end{array}$ & $\begin{array}{c}0.00 \\
(0.00)\end{array}$ & \\
\hline 25 & $\begin{array}{l}R, \text { dapsilis } \\
(\mathbb{N}=15)\end{array}$ & $\begin{array}{c}5.80 \\
(4.58- \\
7.10)\end{array}$ & $\begin{array}{c}5.67 \\
(4.78- \\
7.29)\end{array}$ & $\begin{array}{c}5.65 \\
(4.78- \\
7.54)\end{array}$ & $\begin{array}{c}3.60 \\
(2.29- \\
6.04)\end{array}$ & $\begin{array}{c}4.31 \\
(3.25- \\
5.47)\end{array}$ & $\begin{array}{c}3.45 \\
(2.48- \\
4.50)\end{array}$ & $\begin{array}{c}2.49 \\
(1.72- \\
3.42)\end{array}$ & $\begin{array}{c}2.72 \\
(1.53- \\
3.88)\end{array}$ & $\begin{array}{c}2.90 \\
(2.29- \\
3.56)\end{array}$ & $\begin{array}{c}2.98 \\
(2.29- \\
3.84)\end{array}$ & $\begin{array}{c}3.19 \\
(2.30- \\
4.33)\end{array}$ & $\begin{array}{c}2.31 \\
(1.33- \\
3.14)\end{array}$ & $\begin{array}{r}1.22 \\
(0.00- \\
2.60)\end{array}$ \\
\hline
\end{tabular}


Several populations of Rhinella marina from south of the Amazon River seem to have similar mitochondrial lineages as $R$. diptycha, in contrast to populations northward. As nuclear loci of specimens of both populations of $R$. marina were similar, and divergent from $R$. diptycha, the occurrence of an extensive mtDNA unidirectional introgression from $R$. diptycha into $R$. marina was hypothesized (Sequeira et al., 2011). However, this hypothesis was not conclusively corroborated in a subsequent study because an additional mitochondrial clade, found for some populations of $R$. marina, obscured the direction of the introgression between these species (Vallinoto et al., 2017). A similar situation of possible unidirectional mitochondrial introgression from $R$. dorbignyi to R. bernardoi was reported by Pereyra et al. (2016a). Evidence that supports this hypothesis comes from the well-supported incongruence between the independent analyses of the mitochondrial and nuclear genes: $R$. bernardoi is deeply nested within $R$. dorbignyi in the mitochondrial analysis, but not in the nuclear analysis.

Our results from independent mitochondrial and nuclear analyses (rMD and rND, respectively) also show incongruence in the position of the specimens of Rhinella horribilis s.l. We recover this species deeply nested within (morphologically similar) species of the $R$. marina Group in the rND analysis, whereas in the rMD analysis it is recovered as sister of all the species of the R. crucifer $+R$. marina Groups. Another striking characteristic of this case of hybridization is the origin of these mitochondria, which is not traceable to any known extant species. These particular forms of deep mitochondrial divergence were denominated "ghost introgressions" (see Zhang et al., 2019). This kind of event involving deep mitochondrial divergence that implies past mitochondrial introgression from an unknown and not closely related species is uncommon in anurans. Historical interspecific introgressions events were reported in several groups of Anura: Ameerega (Dendrobatidae; Brown and Twomey, 2009); Anaxyrus, Bufo, and Bufotes (Bufonidae; Malmos et al.,
2001; Yamazaki et al., 2008; Colliard et al., 2010; Dufresnes et al., 2019); Bombina (Bombinatoridae; Hofman and Szymura, 2007; De Cahsan et al., 2019); Dyscophus (Microhylidae; OrozcoterWengel et al., 2013); Hyla (Hylidae; Lamb and Avise, 1986; Bryson et al., 2010, 2014; Klymus et al., 2010); Mantella (Mantellidae; Crottini et al., 2019); Pelophylax and Rana (Ranidae; Liu et al., 2010; Zhou et al., 2012; Eto et al., 2013); Quasipaa (Dicroglossidae; Zhang et al., 2018); and Scutiger (Megophryidae; Chen et al., 2009). However, most of these events (except in Bombina, Bufotes, Quasipaa, and Scutiger) occurred among closely related species. Another striking characteristic of this phenomenon in Rhinella horribilis s.l. is that after the ancient introgression, the GIM (i.e., the mitochondrial DNA) diversified into two divergent clades (UPDs $>3.33 \%$ ). We consider most plausible the hypothesis that these mitochondrial clades represent two different species ( $R$. horribilis s.s. and Rhinella sp. 1) that are not fully detectable (e.g., recovered as monophyletic) with our limited nuclear dataset. More intense genomic and phylogeographic sampling will be necessary to eventually solve the taxonomic status and puzzling history of $R$. horribilis and its lineages.

The reports of hybridization and mitochondrial introgression in Rhinella suggest the need for an extensive and careful exploration of these phenomena in other lineages of Bufonidae. The particular reproductive biology (i.e., scramble competition), the occurrence of broad sympatric areas between related species, and genetic features (i.e., complete reproductive isolation with relatively high levels of genetic divergence; Malone and Fontenot, 2008) of many bufonids may facilitate the occurrence of these phenomena. The identification of foreign mitochondrial genomes is particularly relevant to avoid errors both in phylogeographic and taxonomic studies (especially DNA barcoding studies) and phylogenetic inferences (Ballard and Whitlock, 2004; Alves et al., 2006; Obertegger et al., 2018; Barley et al., 2019). Moreover, the identification of mitochondrial introgressions will serve, among 
other things, as a base for future studies on adaptive coevolution between these organelles and the nuclear components of the oxidative metabolism of the cell (Hill, 2019).

\section{Comments on the Phenoty pic Evidence CONSIDERED FOR RHINELLA}

Our phenotypic sampling results in some synapomorphic and/or diagnostic characters for several internal clades of Rhinella, including most of the species groups. However, an evaluation of the available information for the character systems used clearly shows large gaps in the knowledge within each species group/clades (see fig. 15).

In general, there is relatively more information available for species of the Rhinella marina Clade. Within the $R$. margaritifera Clade, characters on adult osteology and musculature, natural history, and larval morphology are poorly known, and characters of larval chondrocranium, cytogenetics, and embryonic morphology are virtually unknown. This is a source of ambiguity in the reconstruction of ancestral character states for many characters that optimize in more inclusive nodes (e.g., oviposition mode within the $R$. festae and $R$. veraguensis Groups).

With the exceptions of foot and hand musculature, external larval, and embryonic morphologies described for several species of Rhinella (e.g., Mercês et al., 2009; Tolledo and Toledo, 2010; Blotto et al., 2014; Vera Candioti et al., 2016, 2020; Grosso et al., 2020; B.L.B., personal obs.), detailed descriptions considering ontogenetic variation, sexual dimorphism, and interpopulation variations are still largely necessary. It must be noticed that these and many other character systems are promising as additional sources of evidence to be included in future phylogenetic analyses. Some examples of variation within species of Rhinella were reported on bioacoustics (W.F. Martin, 1972; De la Riva et al., 1996; Guerra et al., 2011; Andrade et al., 2015; Valencia-Zuleta et al., 2020); integument and parotoid macroglands structure (O'Donohoe et al., 2019); anatomy of urogenital and digestive systems (Stohler, 1932; Lynch and Renjifo, 1990; but see Grant, 2000); clutch and egg size (Liedtke et al., 2014; Pereyra et al., 2015); mandibular, pelvic, and thigh musculature (Noble, 1922; Limeses, 1964, 1965; Trueb, 1971; Winokur and Hillyard, 1992; Grant and Bolívar-G., 2014); and secretions (Cei et al., 1968; Maciel et al., 2006; Rodríguez et al., 2017). An inclusive sampling considering all these characters will contribute to the study of patterns of evolution of different character systems and their functional and adaptive significance.

The Fossil Record of Rhinella and CaliBRATION POINTS

As is common for most neobatrachian anuran families, the pre-Pleistocene fossil record of Bufonidae is deficient, and most of these specimens lack an apomorphy-based diagnosis to unambiguously assign them to particular nodes or species (see Parham et al., 2012). The currently known pre-Pleistocene fossils of Rhinella are phylogenetically concentrated within the $R$. marina Group: (1) $R$. arenarum (as $R$. pisanoi) from Pliocene outcrops (3.9-3.2 Ma) of coastal Buenos Aires Province, Argentina (Casamiquela, 1967; Pérez-Ben et al., 2014); (2) R. loba, an extinct species putatively related to $R$. arenarum, from the late Pliocene (4.5$3.2 \mathrm{Ma}$ ) from the Chapadmalal Formation of Argentina (Pérez-Ben et al., 2019); (3) R. marina from the mid Miocene (13.8-11.8 Ma) from $\mathrm{La}$ Venta fauna of Colombia (Estes and Wassersug, 1963); and (4) R. aff. arenarum and Rhinella sp. marina Group from the upper Oligocene (29-26 Ma) of Salla, Bolivia (Báez and Nicoli, 2004). Another fossil from the upper Paleocene (59.2-56 Ma) from Itaboraí, Brazil (Estes, 1970) was also considered as related to some of the South American species groups of the former Bufo, but all proposed associations are vague and tentative (see Estes, 1970; Estes and Reig, 1973; Báez and Gasparini, 1977); even an association with Rhinella is controversial. Only the Miocene specimen of $R$. marina has been used as a calibration point (along with few other non-Rhinella bufonid fossils) in divergence dating analyses of Bufonidae or its inter- 


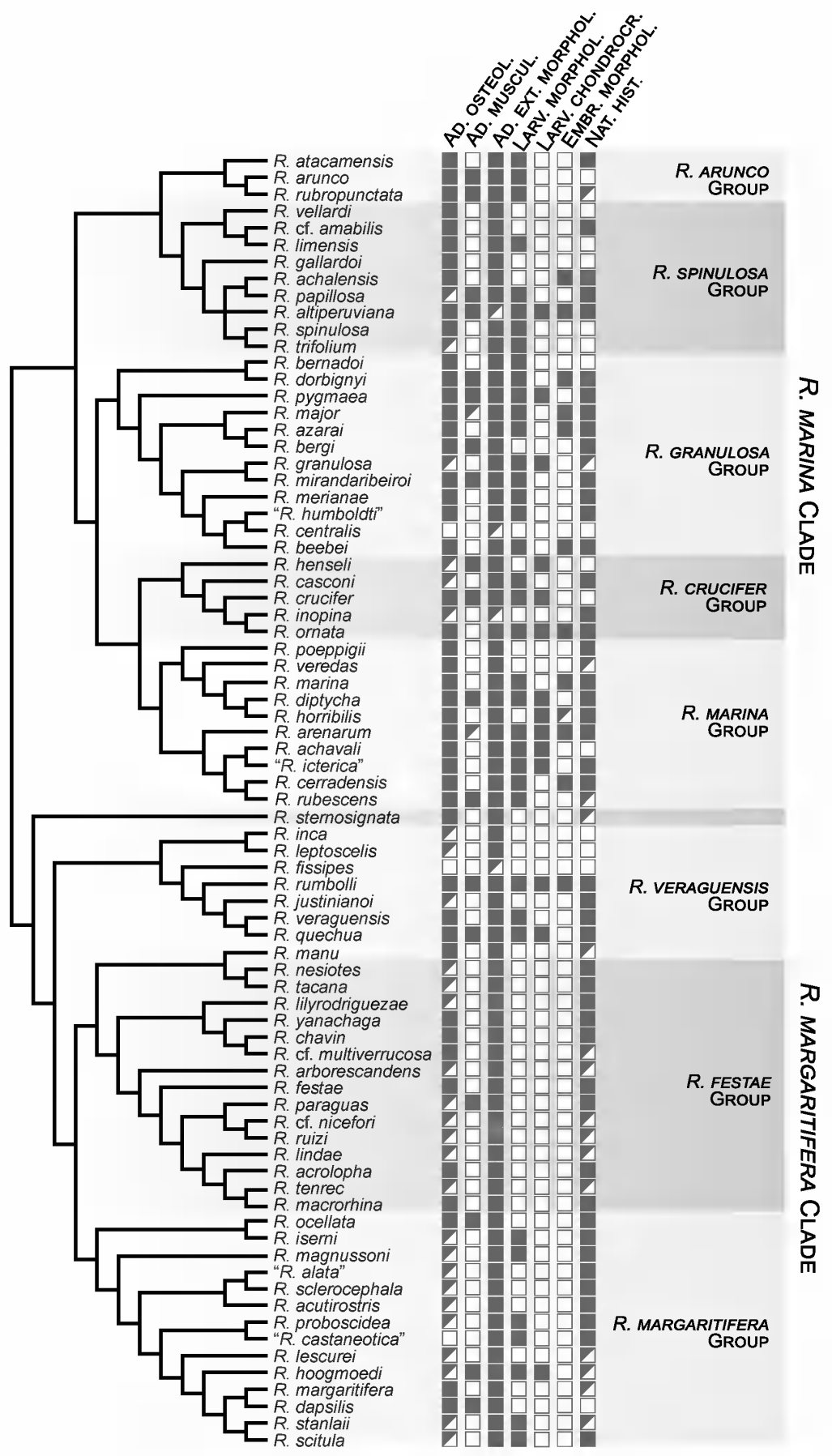

FIG. 15. Main character systems scored for each included species of Rhinella. References: Filled squares, most characters of the considered character system scored ( $>50 \%$ ); half-filled squares, most characters not scored $(<40 \%)$; empty squares, no character scored. Abbreviations: Ad. ext. morphol., Adult external morphology; Ad. musc., Adult musculature; Ad. osteol., Adult osteology; Emb. morphol., Embryonic morphology; Larv. chondrocr., Larval chondrocranium; Larv. morphol., Larval external morphology; Nat. hist., Natural history. 
nal clades (e.g., Pramuk et al., 2008; Maciel et al., 2010; van Bocxlaer et al., 2010; Liedtke et al., 2016, 2017). These studies differ in the sampled taxa and genes, and their results are not fully congruent, but the divergence-time estimates, considering relaxed molecular clocks and similar calibration points, indicate a split time between Rhinella and Anaxyrus + Incilius around $41 \mathrm{Ma}$ (34-47 Ma; Pramuk et al., 2008) and 38.7 Ma (28.5-51.8; van Bocxlaer et al,, 2010). However, the absence of an understanding of the interspecific osteological variation in species of Rhinella and the absence of an apomorphy-based determination of the fossils could result in the association of fossils to a lower-level taxon than the data can demonstrate (see Bever, 2005; Parham et al., 2012). Consequently, a critical reexamination of the available pre-Pleistocene fossils of Rhinella, along with an extensive study of living species of all the species groups is necessary before their defensible use as calibration points in divergence dating analyses. If material from Itaboraí can be unambiguously associated with Rhinella, its inclusion will provide a crucial point of calibration that could modify extensively our current understanding of the patterns of diversification of Rhinella and also of Bufonidae.

\section{CONCLUSIONS}

Our results provide a general framework for better understanding the evolution and taxonomy of Rhinella and its internal clades. The main results of our work include: (1) the generation of a well-supported phylogenetic hypothesis of the genus resulting from a total evidence analysis of most of its specific diversity, (2) the redefinition and morphological diagnosis of its species groups, (3) the demonstration of hybridization and mitochondrial introgression between some species, and (4) evaluation of the taxonomic status of several species. Nevertheless, many challenges are still pending. For example: (1) the taxonomic revision of many clades, including the designation of neotypes for several taxa; (2) the evaluation of the ontogenetic and intersexual variation in several problematic taxa; (3) the use of denser gene sampling (with high throughput sequencing) to better understand the evolutionary relationships in poorly supported clades and evaluate the role of the introgressive hybridization in the evolution of some lineages of Rhinella; and (4) the incorporation of more phenotypic characters to better understand their evolution in this group and define many morphologically and ecologically diverse clades of the genus. Future studies addressing these problems would result in crucial contributions in the knowledge of the diversity of Rhinella.

\section{ACKNOWLEDGMENTS}

We sincerely thank José M. Padial (Bronx Community College, New York); Thelia M. Céspedes Alejabo (CINBIOTYC, Peru); Guarino Colli and Helga Wiederhecker (Coleção Herpetológica da Universidade de Brasília, Brazil); Jimena Grosso (Fundación Miguel Lillo, Argentina); Juan M. Daza (Grupo Herpetológico de Antioquia, Colombia); Diego A. Barrasso and Leonardo Cotichelli (Instituto de Diversidad y Evolución Austral, Centro Nacional Patagónico, Argentina); Albertina P. Lima (Instituto Nacional de Pesquisas da Amazônia, Brazil); Antoine Fouquet (Laboratoire Evolution \& Diversité Biologique, Université Toulouse, France); Juan M. Boeris, Andrés E. Brunetti, Dario E. Cardozo, and Juan M. Ferro (Laboratorio de Genética Evolutiva, Instituto de Biología Subtropical, Argentina); Sebastián Barrionuevo, Nadia G. Cervino, Laura Nicoli, and Santiago J. Nenda (Museo Argentino de Ciencias Naturales, Argentina); César Aguilar (Museo de Historia Natural, Universidad Nacional Mayor de San Marcos, Lima, Perú); Beatriz Álvarez Dorda and Isabel Rey Fraile (Museo Nacional de Ciencias Naturales, Spain); personnel of the herpetological collection of Museo de Biodiversidad (MUBI, Perú); Andrés Pansonato and Tiago Pezzuti (Universidade Federal de Minas Gerais, Brazil); Francisco Brusquetti, Ariadne Fares Sabbag, and Mariana Lyra (Universidad Estadual Paulista, Brazil); and Pedro H. Dias, Juliana Jordão, Julia S. Parreiras, Paulo D.P. Pinheiro, and Miguel Rodrigues (Universidade de São Paulo, Brazil) for sharing with us specimens, tissue samples and/or 
DNA sequences. For access to collections and specimen loans we thank Ariane Silva and Fernanda Werneck (Instituto Nacional de Pesquisas da Amazonia, Brazil); Rafe Brown, Richard Glor, Linda Trueb, and Luke Welton (Kansas University, Lawrence); Marta Calvo (Museo Nacional de Ciencias Naturales, Spain); Aline Staskowian Benetti (Museu de Zoologia da Universidade de São Paulo, Brazil); Carol Spencer and Natasha Stepanova (Museum of Vertebrate Zoology, Berkeley, California); and Eric Smith (University of Texas at Arlington). Pedro H. Dias; Ana Duport Bru, M. Laura Ponssa, and M. Florencia Vera Candioti (Fundación Miguel Lillo, Argentina); and Marinus S. Hoogmoed (Museu Paraense Emilio Goeldi, Brazil), contributed with valuable information on taxonomy or morphology of several specimens. Michael Jowers (Porto University) helped us to clarify the identity of some sequences of $R$. beebei and R. humboldti. M. Jimena Gómez Fernández, M. Daniela Pereyra, and Araceli Seiffe (Museo Argentino de Ciencias Naturales, Argentina) provided technical laboratory assistance. Several colleagues kindly shared with us photographs and/or data of species of Rhinella: César L. Barrio-Amorós (R. sternosignata), Michelle Castellanos ( $R$. sclerocephala), Andrés Cecconi $(R$. diptycha), Pedro H. Dias (R. acutirostris), José Gerardo Espinoza (Rhinella sp. 14), Peter Janzen (R. crucifer), Konstantin D. Milto (type material of R. margaritifera), Arturo Muñoz (R. quechua), Roberto L.M. Novaes ( $R$. icterica), Mirco Solé ( $R$. hoogmoedi), Mauro Texeira Jr. (R. ornata), Rodrigo Tinoco $(R$. ocellata), Vicente Valdés Guzman $(R$. atacamensis), and Mario Yañez-Muñoz ( $R$. cf. roqueana). David Blackburn, contributors, and the staff of Morphosource (Duke Library Digital Repository) very kindly made available $\mu \mathrm{CT}$ images of relevant species. TNT was provided free by the Willi Hennig Society. We thank Consejo Nacional de Investigaciones Científicas y Técnicas (CONICET), ANPCyT, Fundação de Amparo à Pesquisa do Estado de São Paulo for financial support: PIP 11220110100889; PICT 2013-0404, 2015-0813, 2015-0820, 2015-2381, 2017-2437, and 2018-3349; and grants \#2012/10000-5, \#2013/20423-3, \#2013/50741-7, \#2014/03585-2, \#2015/11237-7,
\#2016/25070-0, and \#2018/15425-0 from São Paulo Research Foundation (FAPESP). M.R. was supported by a PNPD post-doctoral fellowship from the Brazilian Coordenação de Aperfeiçoamento de Pessoal de Nível Superior (CAPES grant 2016.1.263.41.6). J.M. thanks Ministry of Culture of the Czech Republic (grant DKRVO 2021/6.VI.c National Museum Prague, 00023272). S.C.F. was supported by $\mathrm{CNPq}$ (grant 312744/ 2017-0) and the PrInt program of Coordenação de Aperfeiçoamento de Pessoal de Nivel Superior, CAPES, Brazil (grant 88887.508359/2020-00). T.G. was supported by research fellowships from the Brazilian Conselho Nacional de Desenvolvimento Científico e Tecnológico (CNPq grant 306823/2017-9). C.F.B.H. thanks $\mathrm{CNPq}$ for a research fellowship (grant 306623/2018-8). The Centro de Estudos de Insetos Sociais, I.B., UNESP, Rio Claro allowed access to its molecular laboratory facilities for the production of some sequences used in this study. Field and laboratory work in Ecuador were funded by Secretaría Nacional de Educación Superior, Ciencia, Tecnología e Innovación del Ecuador SENESCYT (Arca de Noé initiative; S.R.R. and Omar Torres principal investigators) and grants from Pontificia Universidad Católica del Ecuador, Dirección General Académica. B.L.B. acknowledges Esteban O. Lavilla for his support during initial studies on the R. spinulosa Group. We greatly appreciate the critical reviews of the manuscript by Aaron Bauer and Joseph Mendelson.

\section{REFERENCES}

Abbott, R.J., N.H. Barton, and J.M. Good. 2016. Genomics of hybridization and its evolutionary consequences. Molecular Ecology 25: 2325-2332.

Abel, J.J., and D.I. Macht. 1912. Two crystalline pharmacological agents obtained from the tropical toad, Bufo agua. Journal of Pharmacology and Experimental Therapeutics 3: 319-377.

Aberer, A.J., D. Krompass, and A. Stamatakis. 2013. Pruning rogue taxa improves phylogenetic accuracy: an efficient algorithm and webservice. Systematic Biology 62: 162-166.

Acevedo, A.A., M. Lampo, and R. Cipriani. 2016. The cane or marine toad, Rhinella marina (Anura, 
Bufonidae): two genetically and morphologically distinct species. Zootaxa 4103: 574-586.

Acosta-Galvis, A.R. 2012a. Anfibios de los enclaves secos en la ecorregión de la Tatacoa y su área de influencia. Biota Colombiana 13: 182-2010.

Acosta-Galvis, A.R. 2012b. Anfibios de los enclaves secos del área de influencia de los Montes de María y la Ciénaga de la Caimanera en el Departamento de Sucre. Biota Colombiana 13: 211-231.

Acosta-Galvis, A.R., C. Huertas, and M.A. Rada. 2006. Aproximación al conocimiento de los anfibios en una localidad del Magdalena Medio (Departamento de Caldas, Colombia). Revista de la Academia Colombiana de Ciencias Exactas Físicas y Naturales 30: 291-303.

Aguayo, R., E.O. Lavilla, M.F. Vera Candioti, and T. Camacho. 2009. Living in fast-flowing water: morphology of the gastromyzophorous tadpole of the bufonid Rhinella quechua ( $R$. veraguensis group). Journal of Morphology 270: 1431-1442.

Aguilar, C., and R. Gamarra. 2004. Descripción de dos renacuajos y una clave para las larvas conocidas del grupo Bufo spinulosus (Anura: Bufonidae) de Perú. Revista Peruana de Biología 11: 31-36.

Ahmad, F., and M.I. Alam. 2015. An observation of winter breeding by two anurans from Bangladesh. IRCF Reptiles \& Amphibians 22: 29-31.

Alcalde, L. 2017. Osteological development and homology of the prenasal bones in the Neotropical toad Rhinella fernandezae (Anura: Bufonidae). Zoologischer Anzeiger 268: 96-101.

Alonso, R., A.J. Crawford, and E. Bermingham. 2012. Molecular phylogeny of an endemic radiation of Cuban toads (Bufonidae: Peltophryne) based on mitocondrial and nuclear genes. Journal of Biogeography 39: 434-451.

Altig, R., and R.W. McDiarmid. 1999. Body plan: development and morphology. In R.W. McDiarmid and R. Altig (editors), Tadpoles: the biology of the anuran larvae: 24-51. Chicago: University of Chicago Press.

Altig, R., and R.W. McDiarmid. 2007. Morphological diversity and evolution of egg and clutch structure in amphibians. Herpetological Monographs 21: 1-32.

Altig, R., and R.W. McDiarmid. 2015. Handbook of larval amphibians of the United States and Canada: Cornell University Press.

Alves, P., et al. 2006. Hares on thin ice: introgression of mitochondrial DNA in hares and its implications for recent phylogenetic analyses. Molecular Phylogenetics and Evolution 40: 640-641.
Andrade, S.P., C.F. Rocha, E.P. Victor-Junior, and W. Vaz-Silva. 2015. Advertisement call of Rhinella inopina Vaz-Silva, Valdujo \& Pombal, 2012 (Anura: Bufonidae) from the type-locality, northeastern Goiás state, central Brazil. Zootaxa 3931: 448-450.

Angulo, A., and C. Aguilar. 2003. The tadpole of Bufo limensis (Werner, 1901) (Anura: Bufonidae). Amphibia-Reptilia 24: 400-405.

Araujo-Vieira, K., et al. 2019. A total evidence analysis of the phylogeny of hatchet-faced treefrogs (Anura: Hylidae: Sphaenorhynchus). Cladistics 35: 469-486.

Arruda, L.F., M.A.A. Peixoto, C.S. Guimarães, J.V.A. Lacerda, and R.N. Feio. 2014. New state record and geographic distribution map of Rhinella inopina Vaz-Silva, Valdujo \& Pombal, 2012 (Anura: Bufonidae). Check List 10: 395-396.

Ávila, R.W., A. Pansonato, and C. Strüssmann. 2010. A new species of the Rhinella margaritifera group (Anura: Bufonidae) from Brazilian Pantanal. Zootaxa 2339: 57-68.

Ávila, R.W., et al. 2018. On Rhinella gildae Vaz-Silva, Maciel, Bastos \& Pombal 2015 (Anura: Bufonidae): phylogenetic relationship, morphological variation, advertisement and release calls and geographic distribution. Zootaxa 4462: 274-290.

Ávila-Pires, T.C.S., M.S. Hoogmoed, and W.A. Rocha. 2010. Notes on the vertebrates of northern Pará, Brazil: a forgotten part of the Guianan region. I. Herpetofauna. Boletim do Museu Paraense Emílio Goeldi, Ciências Naturais 5: 13-112.

Avise, J. 2008. Clonality: the genetics, ecology, and evolution of sexual abstinence in vertebrate animals. New York: Oxford University Press.

Azevedo, M.F.C., F. Foresti, P.R.R. Ramos, and J. Jim. 2003. Comparative cytogenetic studies of Bufo ictericus, B. paracnemis (Amphibia, Anura) and an intermediate form in sympatry. Genetics and Molecular Biology 26: 289-294.

Baack, E.J., and L.H. Rieseberg. 2007. A genomic view of introgression and hybrid speciation. Current Opinion in Genetics and Development 17: 513-518.

Bacher, S., et al. 2018. Socio-economic impact classification of alien taxa (SEICAT). Methods in Ecology and Evolution 9: 159-168.

Báez, A.M., and N.G. Basso. 1996. The earliest known frogs of the Jurassic of South America: review and cladistic appraisal of their relationships. Müncher Geowissenschaftliche Abhandlungen (A) 30: 131-158.

Báez, A.M., and Z.B. Gasparini. 1977. Orígenes y evolución de los anfibios y reptiles del Cenozoico de América del Sur. Acta Geológica Lilloana 14: 149-232. 
Báez, A.M., and L. Nicoli. 2004. Bufonid toads from the late Oligocene beds of Salla, Bolivia. Journal of Vertebrate Paleontology 24: 73-79.

Báez, A.M., R.O. Gómez, and M.L. Taglioretti. 2012. The archaic ilial morphology of an enigmatic pipid frog from the upper pleistocene of the South American Pampas. Journal of Vertebrate Paleontology 32: 304-314.

Baldissera, F.A., R.F. Batistic, and C.F.B. Haddad. 1999. Cytotaxonomic considerations with the description of two new NOR locations for South American toads, genus Bufo (Anura: Bufonidae). AmphibiaReptilia 20: 413-420.

Baldissera, F.A., U. Caramaschi, and C.F.B. Haddad. 2004. Review of the Bufo crucifer species group, with descriptions of two new related species (Amphibia, Anura, Bufonidae). Arquivos do Museu Nacional do Rio de Janeiro 62: 255-282.

Baldo, D. and N.G. Basso. 2004. New species of Melanophryniscus Gallardo, 1961 (Anura: Bufonidae), with comments on the species of the genus reported for Misiones, northeastern Argentina. Journal of Herpetology 38: 393-403.

Baldo, D., et al. 2012. A cytotaxonomic survey of the genus Melanophryniscus Gallardo, 1961 (Anura: Bufonidae). Journal of Herpetology 46: 25-32.

Baldo, D., et al. 2014. Comparative morphology of pond, stream and phytotelm-dwelling tadpoles of the South American redbelly toads (Anura: Bufonidae: Melanophryniscus). Biological Journal of the Linnean Society 112: 417-441.

Balinsky, B.I. 1969. The reproductive ecology of amphibians of the Transvaal Highveld. Zoologica Africana 4: 37-93.

Ballard, J.W.O., and M.C. Whitlock. 2004. The incomplete natural history of mitochondria. Molecular Ecology 13: 729-744.

Bandeira, L.N., J. Alexandrino, C.F.B. Haddad, and M.T.C. Thomé. 2016. Geographical variation in head shape of a Neotropical group of toads: the role of physical environment and relatedness. Zoological Journal of the Linnean Society 179: 354-376.

Barisone, G.A., J.L. Hedrick, and M.O. Cabada. 2002. Vitelline envelope of Bufo arenarum: biochemical and biological characterization. Biology of Reproduction 66: 1203-1209.

Barley, A.J., et al. 2019. Complex patterns of hybridization and introgression across evolutionary timescales in Mexican whiptail lizards (Aspidoscelis). Molecular Phylogenetics and Evolution 132: 284295.
Barrio-Amorós, C.L., F.J.M. Rojas-Runjaic, and J.C. Señaris. 2019. Catalogue of the amphibians of Venezuela: illustrated and annotated species list, distribution, and conservation. Amphibian and Reptile Conservation 13: 1-198.

Barrionuevo, J.S. 2017. Frogs at the summits: phylogeny of the Andean frogs of the genus Telmatobius (Anura, Telmatobiidae) based on phenotypic characters. Cladistics 33: 41-68.

Bessa-Silva, A., et al. 2020. The roles of vicariance and dispersal in the differentiation of two species of the Rhinella marina species complex. Molecular Phylogenetics and Evolution 145: 106713.

Betto-Colliard, C., S. Hofmann, R. Sermier, N. Perrin, and M. Stöck. 2018. Profound genetic divergence and asymmetric parental genome contributions as hallmarks of hybrid speciation in polyploid toads. Proceedings of the Royal Society B, Biological Sciences 285: 20172667.

Beukema, W., et al. 2013. Review of the systematics, distribution, biogeography and natural history of Moroccan amphibians. Zootaxa 3661: 1-60.

Bever, G.S. 2005. Variation in the ilium of North American Bufo (Lissamphibia; Anura) and its implications for species-level identification of fragmentary anuran fossils. Journal of Vertebrate Paleontology 25: $548-560$.

Biju, S.D., and F. Bossuyt. 2003. New frog family from India reveals an ancient biogeographical link with the Seychelles. Nature 425: 711-714.

Birstein, V.J., and A.L. Mazin. 1982. Chromosomal polymorphism of Bufo bufo: karyotype and c-banding pattern of $B$. b. verrucosissima. Genetica 59: 93-98.

Blair, W.F. 1958. Mating call in the speciation of anuran amphibians. American Naturalist 92: 27-51.

Blair, W.F. 1972. Evolution in the genus Bufo. Austin: University of Texas Press.

Blotto, B.L., M.O. Pereyra, and D. Baldo. 2014. The tadpole of Rhinella azarai (Gallardo, 1965) with comments on larval morphology in the Rhinella granulosa species group (Anura: Bufonidae). Journal of Herpetology 48: 434-438.

Blotto, B.L., M.O. Pereyra, J. Faivovich, P.H.D.S. Dias, and T. Grant. 2017. Concentrated evolutionary novelties in the foot musculature of Odontophrynidae (Anura: Neobatrachia), with comments on adaptations for burrowing. Zootaxa 4258: 425-442.

Blotto, B.L., M.O. Pereyra, T. Grant, and J. Faivovich. 2020. Hand and foot musculature of Anura: structure, homology, terminology, and synapomorphies 
for major clades. Bulletin of the American Museum of Natural History 443: 1-155.

Blotto, B.L., et al. 2021. The phylogeny of the casqueheaded treefrogs (Hylidae: Hylinae: Lophyohylini). Cladistics 37: 36-72.

Bock, W.J., and C.R. Shear. 1972. A staining method for gross dissection of vertebrate muscles. Anatomischer Anzeiger 130: 222-227.

Bokermann, W.C.A. 1967. Notas sôbre a distribuição de Bufo granulosus Spix, 1824 na Amazônia e descrição de uma subespécie nova (Amphibia, Bufonidae). In H. Lent (editor) Atas do simpósio sôbre a biota amazônica: 103-109. Rio de Janeiro, Brazil: Conselho Nacional de Pesquisas.

Bonnet, T., R. Leblois, F. Rousset, and P.A. Crochet. 2017. A reassessment of explanations for discordant introgressions of mitochondrial and nuclear genomes. Evolution 71: 2140-2158.

Borteiro, C., F. Kolenc, M. Tedros, and C. Prigioni. 2006. The tadpole of Chaunus dorbignyi (Duméril \& Bibron) (Anura, Bufonidae). Zootaxa 1308: 49-62.

Bossuyt, F., and M.C. Milinkovitch. 2000. Convergent adaptive radiations in Madagascan and Asian ranid frogs reveal covariation between larval and adult traits. Proceedings of the National Academy of Sciences 97: 6585-6590.

Boulenger, C.L. 1910. On certain subcutaneous fatbodies in toads of the genus Bufo. Proceedings of the Zoological Society of London 80: 379-384.

Boulenger, G.A. 1880. On the Palaearctic and Aethiopian species of Bufo. Proceedings of the Zoological Society of London 1880: 545-574.

Boulenger, G.A. 1882. Catalogue of the Batrachia Salientia s. Ecaudata in the collection of the British Museum, 2nd ed., London: Taylor and Francis.

Boulenger, G.A. 1892. An account of the reptiles and batrachians collected by Mr. C. Hose on Mt. Dulit, Borneo. Proceedings of the Zoological Society of London: 505-508.

Boulenger, G.A. 1897. The tailless batrachians of Europe. Parts I and II. London: Ray Society.

Boulenger, G.A. 1903. Descriptions of new batrachians in the British Museum. Annals and Magazine of Natural History (ser. 7) 12: 552-557.

Boulenger, G.A. 1912. Descriptions of new batrachians from the Andes of South America, preserved in the British Museum. Annals and Magazine of Natural History (ser. 8) 10: 185-191.

Branch, W.R., and H. Braack. 1995. A new toad from paradise. Madoqua 19: 15-23.
Branch, W.R., and H. Braack. 2004. Bufo robinsoni. In L.R. Minter et al. (editors), Atlas and red data book of the frogs of South Africa, Lesotho, and Swaziland: 80-82. Washington, DC: Smithsonian Institution and the Avian Demography Unit.

Brandão, R.A., N.M. Maciel, and A. Sebben. 2007. A new species of Chaunus from central Brazil (Anura; Bufonidae). Journal of Herpetology 41: 309-316.

Brandvain, Y., G.B. Pauly, M.R. May, and M. Turelli. 2014. Explaining Darwin's corollary to Haldane's rule: the role of mitonuclear interactions in asymmetric postzygotic isolation among toads. Genetics 197: 743-747.

Breder, C.M., Jr. 1946. Amphibians and reptiles of the Rio Chucunaque drainage, Darien, Panama, with notes on their life histories and habits. Bulletin of the American Museum of Natural History 86 (8): 375-436.

Brown, F.D., E.M. Del Pino, and G. Krohne. 2002. Bidder's organ in the toad Bufo marinus: effects of orchidectomy on the morphology and expression of lamina-associated polypeptide 2. Development, Growth and Differentiation 44: 527-535.

Brown, J.L., and E.Twomey. 2009. Complicated histories: three new species of poison frogs of the genus Ameerega (Anura: Dendrobatidae) from north-central Peru. Zootaxa 2049: 1-38.

Bruschi, D.P., et al. 2019. Comparative cytogenetics of nine populations of the Rhinella genus (Anura: Bufonidae) with highlight on their conservative karyotype. Genetics and Molecular Biology 42: 445-451.

Bryson, R.W., A. Nieto-Montes de Oca, J.R. Jaeger, and B.R. Riddle. 2010. Elucidation of cryptic diversity in a widespread Nearctic treefrog reveals episodes of mitochondrial gene capture as frogs diversified across a dynamic landscape. Evolution 64: 2315-2330.

Bryson, R.W., B.T. Smith, A. Nieto-Montes de Oca, U.O. García-Vázquez, and B.R. Riddle. 2014. The role of mitochondrial introgression in illuminating the evolutionary history of Nearctic treefrogs. Zoological Journal of the Linnean Society 172: 103-116.

Burton, T.C. 1998. Variation in the hand and superficial throat musculature of Neotropical leptodactylid frogs. Herpetologica 54: 53-72.

Burton, T.C. 2001. Variation in the foot muscles of frogs of the family Myobatrachidae. Australian Journal of Zoology 49: 539-559.

Burton, T.C. 2004. Muscles of the pes of hylid frogs. Journal of Morphology 260: 209-233.

Cadle, J.E., and R. Altig. 1991. Two lotic tadpoles from the Andes of southern Peru: Hyla armata and Bufo veraguensis, with notes on the call of Hyla armata 
(Amphibia: Anura: Hylidae and Bufonidae). Studies on Neotropical Fauna and Environment 26: 45-53.

Caldwell, J.P. 1991. A new species of toad in the genus Bufo from Pará, Brazil, with an unusual breeding site. Papéis Avulsos de Zoologia 37 (26): 389-400.

Caldwell, J.P., and D.B. Shepard. 2007. Calling site fidelity and call structure of a Neotropical toad, Rhinella ocellata (Anura: Bufonidae). Journal of Herpetology 41: 611-621.

Campbell, V., and F.-J. Lapointe. 2009. The use and validity of composite taxa in phylogenetic analysis. Systematic Biology 58: 560-572.

Cannatella, D.C. 1986. A new genus of bufonid (Anura) from South America, and phylogenetic relationships of the Neotropical genera. Herpetologica 42: $197-$ 205.

Caramaschi, U., and H. Niemeyer. 2003. Nova especie do complexo de Bufo margaritifer (Laurenti, 1978) do estado do Mato Grosso do Sul, Brasil. Boletim do Museu Nacional do Rio de Janeiro 501: 1-16.

Caramaschi, U., and J.P. Pombal. 2006. A new species of Rhinella Fitzinger, 1826 from the Atlantic Rain Forest, eastern Brazil (Amphibia, Anura, Bufonidae). Papéis Avulsos de Zoologia 46: 251-259.

Carrizo, G.R. 1992. Cuatro especies nuevas de anuros (Bufonidae: Bufo e Hylidae: Hyla) del norte de la Argentina. Cuadernos de Herpetología 7: 14-23.

Carvalho e Silva, A.M.P.T., and S.P. Carvalho e Silva. 1994. Données sur la biologie et description des larvaes de Bufo pygmaeus Myers et Carvalho (Amphibia, Anura, Bufonidae). Revue Française de Aquariologie 21: 53-56.

Casamiquela, R.M. 1967. Sobre un nuevo Bufo fósil de la provincia de Buenos Aires (Argentina). Ameghiniana 5: 161-169.

Cei, J.M. 1962. Batracios de Chile, Santiago: Universidad de Chile.

Cei, J.M. 1972a. Bufo of South America. In W.F. Blair (editor), Evolution in the genus Bufo: 82-92. Austin: University of Texas Press.

Cei, J.M. 1972b. Segregación corológica y procesos de especiación por aislamiento en anfibios de la Pampa de Achala, Córdoba. Acta Zoológica Lilloana 29: 233-246.

Cei, J.M. 1980. Amphibians of Argentina. Monitore Zoologico Italiano 2: 1-609.

Cei, J.M., V. Erspamer, and M. Roseghini. 1968. Taxonomic and evolutionary significance of biogenic amines and polypeptides occurring in amphibian skin. II. Toads of the genera Bufo and Melanophryniscus. Systematic Zoology 17: 232-245.
Ceschin, D.G., N.S. Pires, M.N. Mardirosian, C.I. Lascano, and A. Venturino, A. 2020. The Rhinella arenarum transcriptome: de novo assembly, annotation and gene prediction. Scientific Reports 10: 1-8.

Céspedez, J.A. 2000. Una nueva especie de Bufo del grupo granulosus (Anura: Bufonidae) del nordeste argentino. FACENA 15: 69-82.

Channing, A., M.-O. Rödel, and J. Channing. 2012. Tadpoles of Africa. The biology and identification of all known tadpoles in sub-Saharan Africa. Frankfurt am Main: Edition Chimaira.

Chaparro, J.C., J.B. Pramuk, and A.G. Gluesenkamp. 2007. A new species of arboreal Rhinella (Anura: Bufonidae) from cloud forest of southeastern Peru. Herpetologica 63: 203-212.

Chávez, G., D. Vásquez, and L.Y. Echevarría. 2013. Amphibia, Anura, Bufonidae, Rhinella manu Chaparro, Pramuk and Gluesenkamp, 2007; Rhinella tacana Padial, Reichle, McDiarmid and De la Riva, 2006: distribution extension and country record from southern Peru. Check List 9: 840-843.

Chen, W., K. Bi, and J. Fu. 2009. Frequent mitochondrial gene introgression among high elevation tibetan megophryid frogs revealed by conflicting gene genealogies. Molecular Ecology 18: 28562876.

Cignoni, P., et al. 2008. Meshlab: an open-source mesh processing tool. In V. Scarano, R. De Chiara, and U. Erra (editors), Proceedings of the Eurographics Italian Chapter Conference: 129-136. Salerno, Italy: The Eurographics Association.

Clarke, B.T. 1981. Comparative osteology and evolutionary relationships in the African Raninae (Anura: Ranidae). Monitore Zoologico Italiano 15 (14): 285-331.

Cochran, D.M. 1955. Frogs of southeastern Brazil. Bulletin of the United States National Museum 206: 1-423.

Cochran, D.M., and C.J. Goin. 1970. Frogs of Colombia. Bulletin of the United States National Museum 288: 1-655.

Colliard, C., et al. 2010. Strong reproductive barriers in a narrow hybrid zone of West-Mediterranean green toads (Bufo viridis subgroup) with Plio-Pleistocene divergence. BMC Evolutionary Biology 10: 232.

Cope, E.D. 1862. On some new and little known American Anura. Proceedings of the Academy Natural Sciences of Philadelphia 14: 151-159.

Cope, E.D. 1889. The Batrachia of North America. Bulletin of the United States National Museum 34: $1-525$. 
Córdova, J.H. 1999. On karyomorphs, cladistics and taxonomic status of the Bufo spinulosus species group (Amphibia: Anura) in Peru. Stuttgarter Beitrage zur Naturkunde (ser. A, Biologie) 600: 1-28.

Córdova, J.H., and J. Descailleaux. 1996. Tres cariotipos diferentes y un híbrido en poblaciones naturales de Bufo marinus (Amphibia: Anura) en Perú. Theorema 5: 13-28.

Correa, C., M.A. Mendez, S. Araya, G. Lobos, and R.E. Palma. 2013. A hybrid zone of two toad sister species, Rhinella atacamensis and R. arunco (Anura: Bufonidae), defined by a consistent altitudinal segregation in watersheds. Revista Chilena de Historia Natural 86: 115-125.

Correa, C.L., M.A. Méndez, A. Veloso, and M. Sallaberry. 2012. Genetic and reproductive evidence of natural hybridization between the sister species $R h i$ nella atacamensis and Rhinella arunco (Anura, Bufonidae). Journal of Herpetology 46: 568-578.

Crawford, A.J., K.R. Lips, and E. Bermingham. 2010. Epidemic disease decimates amphibian abundance, species diversity, and evolutionary history in the highlands of central Panama. Proceedings of the National Academy of Sciences of the United States of America 107: 13777-13782.

Crottini, A., P. Orozco-terWengel, F.C. Rabemananjara, J.S. Hauswaldt, and M. Vences. 2019. Mitochondrial introgression, color pattern variation, and severe demographic bottlenecks in three species of Malagasy poison frogs, genus Mantella. Genes 10: 317.

Cusi, J.C., J. Moravec, E. Lehr, and V. Gvoždík. 2017. A new species of semiarboreal toad of the Rhinella festae group (Anura, Bufonidae) from the Cordillera Azul National Park, Peru. ZooKeys 673: 21-47.

Darras, H., and S. Aron. 2015. Introgression of mitochondrial DNA among lineages in a hybridogenetic ant. Biology Letters 11: 20140971.

Darst, C.R., and D.C. Cannatella. 2004. Novel relationships among hyloid frogs inferred from $12 \mathrm{~s}$ and $16 \mathrm{~s}$ mitochondrial DNA sequences. Molecular Phylogenetics and Evolution 31: 462-475.

De la Riva, I. 2002. Taxonomy and distribution of the South American toad Bufo poeppigii Tschudi, 1845 (Amphibia, Anura, Bufonidae). Graellsia 58: 49-57.

De la Riva, I., J. Bosch, and R. Márquez. 1996. Advertisemnt calls of two Bolivian toads (Anura: Bufonidae: Bufo). Herpetological Journal 6: 59-61.

De la Riva, I., J. Köhler, S. Lötters, and S. Reichle. 2000. Ten years of research on Bolivian amphibians: updated checklist, distribution, taxonomic prob- lems, literature and iconography. Revista Española de Herpetología 14: 19-164.

De Cahsan, B., et al. 2019. Introgression of Austrian fire-bellied toads (Bombina bombina) into northern German populations confirmed by complete mitochondrial genomes and transcriptome-wide single nucleotide polymorphisms (SNPs). bioRxiv: 651695. [doi.org/10.1101/651695]

Díaz, L.M., and A. Cádiz. 2008. Guía taxonómica de los anfibios de Cuba. Abc Taxa 4: 1-294.

Díaz-Lameiro, A.M., J.L. Herrera, D. Barber, R. Powell, and F. Bird-Picó. 2010. Peltophryne lemur. Catalogue of American Amphibians and Reptiles (CAAR) 878: 1-7.

Dixon, J.A. 1976. Bufo dapsilis Myers and Carvalho second known specimen and new to Peru. Herpetological Review 7: 172.

Donoso-Barros, R. 1975. Dos larvas de Bufo chilenos no conocidas. Noticiero Mensual del Museo Nacional de Historia Natural 6-7: 230-231.

Drewes, R.C. 1984. A phylogenetic analysis of the Hyperoliidae (Anura): treefrogs of Africa, Madagascar, and the Seychelles islands. Occasional Papers of the California Academy of Sciences 139: 1-70.

Duellman, W.E. 1978. The biology of an equatorial herpetofauna in Amazonian Ecuador. University of Kansas Publications, Museum of Natural History 65: 1-352.

Duellman, W.E. 1999. Distribution patterns of amphibians in South America. In W.E. Duellman (editor), Patterns of distribution of amphibians a global perspective: 255-328. Baltimore: Johns Hopkins University Press.

Duellman, W.E. 2005. Cusco Amazonico. The lives of amphibians and reptiles in an Amazonian rainforest. Ithaca: Cornell University Press.

Duellman, W.E., and J.D. Lynch. 1969. Description of Atelopus tadpoles and their relevance to atelopodid classification. Herpetologica 25: 231-240.

Duellman, W.E., and J.D. Lynch. 1988. Anuran amphibians from the Cordillera de Cutucu, Ecuador. Proceedings of the Academy of Natural Sciences of Philadelphia 140: 125-142.

Duellman, W.E., and R. Schulte. 1992. Description of a new species of Bufo from northern Peru with comments on phenetic groups of South American toads (Anura: Bufonidae). Copeia 1992: 162-172.

Duellman, W.E., and C.A. Toft. 1979. Anurans from the Serranía de Sira, Amazonian Perú: taxonomy and biogeography. Herpetologica 35: 60-70. 
Duellman, W.E., and L. Trueb. 1986. Biology of Amphibians, 1st ed. New York: McGraw-Hill.

Dufresnes, C. et al. 2019. Fifteen shades of green: the evolution of Bufotes toads revisited. Molecular Phylogenetics and Evolution 141: 106615.

Duméril, A.M.C., and Bibron, G. 1841. Erpétologie genérale ou Histoire naturelle complète des reptiles. Vol. 8. Paris: Librarie Enclyclopedique de Roret.

Dunlap, D.G. 1960. The comparative myology of the pelvic appendage in the salientia. Journal of Morphology 106: 1-76.

Edwards, R.J. et al. 2018. Draft genome assembly of the invasive cane toad, Rhinella marina. GigaScience 7: giy095.

Estes, R. 1970. Origin of the recent North American lower vertebrate fauna; an inquiry into the fossil record. Forma et Functio 4: 139-163.

Estes, R., and O.A. Reig. 1973. The early fossil record of frogs: a review of the evidence. In J.L. Vial (editor), Evolutionary biology of the anurans: contemporary research on major problems: 11-36. Columbia: University of Missouri Press.

Estes, R., and R. Wassersug. 1963. A Miocene toad from Colombia, South America. Breviora 193: 1-13.

Estoup, A., M. Beaumont, F. Sennedot, C. Moritz, and J.-M. Cornuet. 2004. Genetic analysis of complex demographic scenarios: spatially expanding populations of the cane toad, Bufo marinus. Evolution 58: 2021-2036.

Estoup, A. et al. 2010. Combining genetic, historical and geographical data to reconstruct the dynamics of bioinvasions: application to the cane toad Bufo marinus. Molecular Ecology Resources 10: 886901.

Eterovick, P.C., and I. Sazima. 1999. Description of the tadpole of Bufo rufus with notes on aggregative behavior. Journal of Herpetology 33: 711-713.

Eto, K., M. Matsui, and T. Sugahara. 2013. Discordance between mitochondrial DNA genealogy and nuclear DNA genetic structure in the two morphotypes of Rana tagoi tagoi (Amphibia: Anura: Ranidae) in the Kinki Region, Japan. Zoological Science 30: 553-559.

Fabrezi, M. 2006. Morphological evolution in Ceratophryinae (Anura, Neobatrachia). Journal of Zoological Systematics and Evolutionary Research 44: 153-166.

Faivovich, J. 2002. A cladistic analysis of Scinax (Anura: Hylidae). Cladistics 18: 367-393.

Faivovich, J., and G. Carrizo. 1997. La identidad de Bufo missionum Berg, 1896 (Anura, Bufonidae). Cuadernos de Herpetología 11: 81-82
Faivovich, J., et al. 2005. Systematic review of the frog family Hylidae, with special reference to Hylinae: phylogenetic analysis and taxonomic revision. Bulletin of the American Museum of Natural History 294: $1-240$.

Farris, J.S. 1983. The logical basis of phylogenetic analysis. In N.I. Platnick and V.A. Funk (editors), Advances in cladistics: proceedings of the third meeting of the Willi Hennig Society: 7-36. New York: Columbia University Press.

Farris, J.S., V.A. Albert, M. Källersjö, D. Lipscomb, and A.G. Kluge. 1996. Parsimony jackknifing outperforms neighbour-joining. Cladistics 12: 99-124.

Feder, J.H. 1979. Natural hybridization and genetic divergence between the toads Bufo boreas and Bufo punctatus. Evolution 33: 1089-1097.

Feller, A., and J.B. Hedges. 1998. Molecular evidence for the early history of living amphibians. Molecular Phylogenetics and Evolution 9: 509-516.

Fenolio, D.B., J.R. Mendelson, and W.W. Lamar. 2012. A new diagnosis and description of variation among adult Rhinella ceratophrys (Boulenger) (Amphibia: Bufonidae), with notes on ecology and distribution. South American Journal of Herpetology 7: 9-15.

Fernández, K. 1927. Sobre la biología y reproducción de batracios argentinos. Segunda parte. Boletín Academia Nacional de Ciencias de Córdoba 29: 271320.

Ferraro, D.P., et al. 2018. Componente 1. Sistemática y diversidad. In M. Vaira, M.S. Akmentins, and E.O. Lavilla (editors), Plan de acción para la conservación de los anfibios de la República Argentina. Cuadernos de Herpetología: 15-19. Tucumán: Asociación Herpetológica Argentina.

Fontenot, B.E., R. Makowsky, and P.T. Chippindale. 2011. Nuclear-mitochondrial discordance and gene flow in a recent radiation of toads. Molecular Phylogenetics and Evolution 59: 66-80.

Ford, L.S. 1990. The phylogenetic position of poisondart frogs (Dendrobatidae): reassessment of the neobatrachian phylogeny with commentary on complex character systems. Ph.D. dissertation, Department of Systematics and Ecology, University of Kansas, Lawrence.

Formas, J.R. 1978. The chromosomes of Bufo rubropunctatus and Bufo chilensis (Anura, Bufonidae) and other species of the spinolosus group. Experientia 34: $452-454$.

Formas, J.R., and E. Pugín. 1978. Tadpoles of Hylorina sylvatica, Eupsophus vittatus, and Bufo rubropunctatus in southern Chile. Herpetologica 34: 355-358. 
Fouquet, A., P. Gaucher, M. Blanc, and C.M. VélezRodríguez. 2007a. Description of two new species of Rhinella (Anura: Bufonidae) from the lowlands of the Guiana Shield. Zootaxa 1663: 17-32.

Fouquet, A., et al. 2007b. Underestimation of species richness in Neotropical frogs revealed by mtDNA analyses. PLoS ONE 2: e1109.

Fouquet, A., et al. 2007c. Revealing cryptic diversity using molecular phylogenetics and phylogeography in frogs of the Scinax ruber and Rhinella margaritifera species groups. Molecular Phylogenetics and Evolution 43: 567-582.

Fouquet, A., et al. 2012a. Multiple quaternary refugia in the eastern Guiana shield revealed by comparative phylogeography of 12 frog species. Systematic Biology 61: 461-489.

Fouquet, A., et al. 2012b. Molecular phylogeny and morphometric analyses reveal deep divergence between Amazonia and Atlantic Forest species of Dendrophryniscus. Molecular Phylogenetics and Evolution 62: 826-838.

Freitas, M.A., E.M. dos Santos, F.O. Amorim, and G.V.L. Almeida. 2018. First record of Rhinella ocellata (Günther, 1858) for the state of Bahia, northeastern Brazil (Anura: Bufonidae). Herpetology Notes 11: 17-18.

Frost, D.R. 2020. Amphibian species of the world: an online reference. Version $6.0 \mathrm{http} / / /$ research.amnh. org/herpetology/amphibia/index.php (18 January 2020).

Frost, D.R., et al. 2006. The amphibian tree of life. Bulletin of the American Museum of Natural History 297: 1-370.

Funk, W.C., M. Caminer, and S.R. Ron. 2011. High levels of cryptic species diversity uncovered in Amazonian frogs. Proceedings of the Royal Society B, Biological Sciences 279: 1806-1814.

Gallardo, J.M. 1957. Las subespecies argentinas de Bufo granulosus Spix. Revista del Museo Argentino de Ciencias Naturales "Bernardino Rivadavia" 3: 337-374.

Gallardo, J.M. 1961. Three new toads from South America: Bufo manicorensis, Bufo spinulosus altiperuvianus and Bufo quechua. Breviora 141: 1-8.

Gallardo, J.M. 1962. A propósito de Bufo variegatus (Günther), sapo del bosque húmedo antartándico, y las otras especies de Bufo neotropicales. Physis 23: 93-102.

Gallardo, J.M. 1965. The species Bufo granulosus Spix (Salientia: Bufonidae) and its geographic variation. Bulletin of the Museum of Comparative Zoology 134: $107-138$
Gallardo, J.M. 1967. Bufo gnustae sp. nov. del grupo de B. ockendeni Boulenger, hallado en la provincia de Jujuy, Argentina. Neotropica 13 (41): 54-56.

Gaupp, E. 1896. A. Ecker's und R. Wiedersheim's anatomie des frosches, part 1. Braunschweig: Friedrich Vieweg und Sohn.

Gergus, E.W., K.B. Malmos, and B.K. Sullivan. 1999. Natural hybridization among distantly related toads (Bufo alvarius, Bufo cognatus, Bufo woodhousii) in central Arizona. Copeia 1999: 281-286.

Giribet, G. 2005. A review of: "TNT: Tree Analysis Using NewTechnology". Systematic Biology 54: 176-178

Goebel, A.M., J.M. Donnelly, and M.E. Atz. 1999. PCR primers and amplification methods for $12 \mathrm{~S}$ ribosomal DNA, the control region, cytochrome oxidase 1 , and cytochrome $\mathrm{b}$ in bufonids and other frogs, and an overview of PCR primers which have amplified DNA in amphibians successfully. Molecular Phylogenetics and Evolution 11: 163-199.

Goebel, A.M., T.A. Ranker, P.S. Corn, and R.G. Olmstead. 2009. Mitochondrial DNA evolution in the Anaxyrus boreas species group. Molecular Phylogenetics and Evolution 50: 209-225.

Goloboff, P.A. 1999. Analyzing large data sets in reasonable times: solutions for composite optima. Cladistics 15: 415-428.

Goloboff, P.A. 2003. Parsimony, likelihood, and simplicity. Cladistics 19: 91-103.

Goloboff, P.A., and S.A. Catalano. 2016. TNT version 1.5 , including a full implementation of phylogenetic morphometrics. Cladistics 32: 221-238.

Goloboff, P.A., and D. Pol. 2005. Parsimony and Bayesian phylogenetics. In V.A. Albert (editor), Parsimony, phylogeny, and genomics: 148-159. Oxford: Oxford University Press.

Goloboff, P.A., and C.A. Szumik. 2015. Identifying unstable taxa: efficient implementation of tripletbased measures of stability, and comparison with Phyutility and RogueNaRok. Molecular Phylogenetics and Evolution 88: 93-104.

Goloboff, P.A., et al. 2003. Improvements to resampling measures of group support. Cladistics 19 : 324-332.

Goloboff, P.A., J.S. Farris, and K.C. Nixon. 2008. TNT, a free program for phylogenetic analysis. Cladistics 24: 774-786.

Gómez, R.O., and G.F. Turazzini. 2016. An overview of the ilium of anurans (Lissamphibia, Salientia), with a critical appraisal of the terminology and primary homology of main ilial features. Journal of Vertebrate Paleontology 36: e1030023 
Gopalakrishnan, S., et al. 2018. Interspecific gene flow shaped the evolution of the genus Canis. Current Biology 28 (21): 3441-3449.

Grandison, A.G.C. 1981. Morphology and phylogenetic position of the west African Didynamipus sjoestedti Andersson, 1903 (Anura Bufonidae). Monitore Zoologico Italiano 15(11): 187-215.

Grant, T. 2000. Una nueva especie de Rhamphophryne (Anura: Bufonidae) de la Cordillera Central de Colombia. Revista de la Academia Colombiana de Ciencias Exactas, Físicas y Naturales 23 (Supl.): 287-292.

Grant, T., and W. Bolívar-G. 2014. A new species of semiarboreal toad with a salamander-like ear (Anura: Bufonidae: Rhinella). Herpetologica 70: 198-210.

Grant, T., et al. 2006. Phylogenetic systematics of dartpoison frogs and their relatives (Amphibia: Athesphatanura: Dendrobatidae). Bulletin of the American Museum of Natural History 299: 1-262.

Graybeal, A. 1997. Phylogenetic relationships of bufonid frogs and tests of alternate macroevolutionary hypotheses characterizing their radiation. Zoological Journal of the Linnean Society 119: 297-338.

Green, D.M. 1996. The bounds of species: Hybridization in the Bufo americanus group of North American toads. Israel Journal of Zoology 42: 95-109.

Green, D., and C. Parent. 2003. Variable and asymmetric introgression in a hybrid zone in the toads, Bufo americanus and Bufo fowleri. Copeia 2003: 34-43.

Grosso, J.R., M.O. Pereyra, F. Vera Candioti, N.M. Maciel, and D. Baldo. 2020. Tadpoles of three species of the Rhinella granulosa group, with a reinterpretation of larval characters. South American Journal of Herpetology 15: 75-84.

Guarnizo, C.E., et al. 2015. DNA barcoding survey of anurans across the eastern cordillera of Colombia and the impact of the andes on cryptic diversity. PLoS ONE 10: e0127312.

Guayara-Barragán, M.G., and M.H. Bernal. 2012. Fecundidad y fertilidad en once especies de anuros colombianos con diferentes modos reproductivos. Caldasia 34: 483-496.

Guerra, C., D. Baldo, S. Rosset, C. Borteiro, and F. Kolenc. 2011. Advertisement and release calls in Neotropical toads of the Rhinella granulosa group and evidence of natural hybridization between $R$. bergi and R. major (Anura: Bufonidae). Zootaxa 3092: 26-42.

Guichenot, A. 1848. Reptilianos. In C. Gay (editor), Historia fisica y politica de Chile. Vol. 2 (Zoología): 1-136. Paris, Maulde and Renou.
Günther, A. 1858a. Catalogue of the Batrachia Salientia in the collection of the British Museum. London: Taylor and Francis.

Günther, A. 1858b. Neue Batrachier in der Sammlung des britischen Museums. Archiv für Naturgeschichte. Berlin 24: 319-328.

Haad, M.B., F. Vera Candioti, and D. Baldo. 2014. The stream tadpoles of Rhinella rumbolli (Anura: Bufonidae). Herpetologica 70: 184-197.

Haas, A. 2003. Phylogeny of frogs as inferred from primarily larval characters (Amphibia: Anura). Cladistics 19: 23-89.

Haas, A., J. Wolter, S.T. Hertwig, and I. Das. 2009. Larval morphologies of three species of stream toads, genus Ansonia (Amphibia: Bufonidae) from east Malaysia (Borneo), with a key to known bornean Ansonia tadpoles. Zootaxa 2302: 1-18.

Haas, W. 2002. Beitrag zum taxonomischen Status von Bufo trifolium Tschudi, 1845 und Bufo spinulosus flavolineatus Vellard, 1959 sowie zur Biologie von Bufo spinulosus Wiegmann, 1834. Salamandra 38: 155-164.

Haddad, C.F.B., G.V. Andrade, and A.J. Cardoso. 1988. Anfíbios anuros no Parque Nacional da Serra da Canastra, Estado de Minas Gerais. Brasil Florestal 64: 9-20.

Haddad, C.F.B., A.J. Cardoso, and L.M. Castanho. 1990. Hibridação natural entre Bufo ictericus e Bufo crucifer (Amphibia: Anura). Revista Brasileira de Biologia 50: 739-744.

Hall, T.A. 1999. Bioedit: a user-friendly biological sequence alignment editor and analysis for Windows 95/98/NT. Nucleic Acid Symposium Series 41: 95-98.

Harper, E.B., G.J. Measey, D.A. Patrick, M. Menegon, and J.R. Vonesh. 2010. Field guide to the amphibians of the eastern arc mountains and coastal forests of Tanzania and Kenya, Nairobi, Kenya: Camerapix Publishers International.

Harvey, M.B., and E.N. Smith. 1993. A new aquatic Bufo (Anura: Bufonidae) from cloud forests in the Serranía de Siberia, Bolivia. Proceedings of the Biological Society of Washington 106: 442-449.

Harvey, M.B., and E.N. Smith. 1994. A new species of Bufo (Anura: Bufonidae) from cloud forest in Bolivia. Herpetologica 50: 32-38.

Hass, C.A., J.F. Dunski, L.R. Maxson, and M.S. Hoogmoed. 1995. Divergent lineages within the Bufo margaritifera complex (Amphibia: Anura; Bufonidae) revealed by albumin immunology. Biotropica 27: $238-249$. 
Hayes, M.P., and D.M. Krempels. 1986. Vocal sac variation among frogs of the genus Rana from western North America. Copeia 1986: 927-936.

Hedges, S.B. 1994. Molecular evidence for the origin of birds. Proceedings of the National Academy of Sciences 91: 2621-2624.

Hensel, R. 1867. Beiträge zur Kenntnis der Wirbelthiere südbrasilens. Archiv für Naturgeschichte 33: 120162.

Hennig, W. 1966. Phylogenetic systematics. Chicago: University of Illinois Press.

Hero, J.-M. 1990. An illustrated key to tadpoles occurring in the central Amazon rainforest, Manaus, Amazonas, Brasil. Amazoniana 11: 201-262.

Heyer, W.R. 1975. A preliminary analysis of the intergeneric relationships of the frog family Leptodactylidae. Smithsonian Contributions to Zoology 199: 1-55.

Heyer, W.R., and D.S. Liem. 1976. Analysis of the intergeneric relationships of the Australian frog family Myobatrachidae. Smithsonian Contributions to Zoology 233: 1-29.

Heyer, W.R. 1978. Systematics of the fuscus group of the frog genus Leptodactylus (Amphibia, Leptodactylidae). Scientific Bulletin, Natural History Museum of Los Los Angeles County 29: 1-85.

Heyer, W.R., A.S. Rand, C.A.G. Cruz, O.L. Peixoto, and C.E. Nelson. 1990. Frogs of Boracéia. Arquivos de Zoologia 31: 231-410.

Hill, G.E. 2019. Reconciling the mitonuclear compatibility species concept with rampant mitochondrial introgression. Integrative and Comparative Biology 59: 912-924.

Hillis, D.M., and R.O. de Sá. 1988. Phylogeny and taxonomy of the Rana palmipes group (Salientia: Ranidae). Herpetological Monographs 2: 1-26.

Hoang, D.T., O. Chernomor, A. von Haeseler, B.Q. Minh, and L.S. Vinh. 2018. UFBoot2: Improving the ultrafast bootstrap approximation. Molecular Biology and Evolution 35: 518-522.

Hock, R.J. 1967. Temperature effect on breeding of the toad, Bufo variegatus, in southern Chile. Copeia 1967: 227-230.

Hödl, W. 1990. Reproductive diversity in Amazonian lowland frogs. Fortschritte der Zoologie 38: 41-60.

Hoegg, S., M. Vences, H. Brinkmann, and A. Meyer. 2004. Phylogeny and comparative substitution rates of frogs inferred from sequences of three nuclear genes. Molecular Biology and Evolution 21: 1188-1200.

Hoff, K.S., A.R. Blaustein, R.W. McDiarmid, and R. Altig. 1999. Behavior: interactions and their conse- quences. In R.W. McDiarmid and R. Altig (editors), Tadpoles: the biology of anuran larvae: 215-239. Chicago: University of Chicago Press.

Hofman, S., and J.M. Szymura. 2007. Limited mitochondrial DNA introgression in a Bombina hybrid zone. Biological Journal of the Linnean Society 91: 295-306.

Hoogmoed, M.S. 1977. On the presence of Bufo nasicus Werner in Guiana, with a redescription of the species on the basis of recently collected material. Zoologische Mededelingen 51: 265-275.

Hoogmoed, M.S. 1986. Biosystematic studies of the Bufo "typhonius" group. A preliminary progress report. In Z. Roček (editor), Studies in herpetology: 147-150. Prague: Charles University.

Hoogmoed, M.S. 1989. South American bufonids (Amphibia: Anura: Bufonidae), an enigma for taxonomists. In X. Fontanet (editor), Treballs d'ictiologia i herpetologia: 167-180. Barcelona: Societat Catalana d'Ictiologia i Herpetologia.

Hoogmoed, M.S. 1990. Biosystematics of South American Bufonidae, with special reference to the Bufo "typhonius" group. In G. Peters and R. Hutterer (editors), Vertebrates in the tropics: 113-123. Bonn: Museum Alexander Koenig.

Houssay, B.A. 1949. Hypophyseal functions in the toad Bufo arenarum Hensel. Quarterly Review of Biology 24: $1-27$

Houssay, B.A., and L. Giusti. 1929. Les fonctions de l'hypophyse et de la région infundibulo-tubérienne chez le crapaud. Comptes Rendus des Séances de la Société de Biologie et de ses Filiales (Paris) 101: 935-938.

Hoyos, J.M., C. Mantilla, D. Galindo, and L. Salgar. 2014. Phylogenetic analysis within the Pristimantis unistrigatus (Anura, Craugastoridae) group based on morphological characters. Caldasia 36: 107-124.

Hudson, C.M., G.P. Brown, K. Stuart, and R. Shine. 2018. Sexual and geographical divergence in head widths of invasive cane toads, Rhinella marina (Anura: Bufonidae), is driven by both rapid evolution and plasticity. Biological Journal of the Linnean Society 124: 188-199.

Inger, R.F. 1960. A review of the oriental toads of the genus Ansonia Stoliczka. Fieldiana Zoology 39: 473503.

Inger, R.F. 1966. The systematics and zoogeography of the Amphibia of Borneo. Sabah: Lun Hing Trading Co.

Inger, R.F. 1972. Bufo of Eurasia. In W.F. Blair (editor), Evolution in the genus Bufo: 102-118. Austin: University of Texas Press. 
Inger, R.F. 1985. Tadpoles of the forested regions of Borneo. Fieldiana Zoology 26: 1-89.

Inger, R.F., and Greenberg, B. 1956. Morphology and seasonal development of sex characters in two sympatric toads. Journal of Morphology 99: 549-574.

IUCN. 2020. The IUCN Red List of threatened species. Version 2020-1.

Jansen, M., R. Bloch, A. Schulze, and M. Pfenninger. 2011. Integrative inventory of Bolivia's lowland anurans reveals hidden diversity. Zoologica Scripta 40: 567-583.

Jetz, W., and R.A. Pyron. 2018. The interplay of past diversification and evolutionary isolation with present imperilment across the amphibian tree of life. Nature Ecology \& Evolution 2: 850.

Jiménez de la Espada, M. 1875. Vertebrados del viaje al Pacífico verificado de 1862 a 1865 por una comisión de naturalistas enviada por el gobierno español. Batracios. Madrid: Imprenta de Miguel Ginesta.

Jolly, C.J., R. Shine, and M.J. Greenlees. 2015. The impact of invasive cane toads on native wildlife in southern Australia. Ecology and Evolution 5: 3879-3894.

Kalyaanamoorthy, S., B.Q. Minh, T.K. Wong, A. von Haeseler, and L.S. Jermiin. 2017. ModelFinder: fast model selection for accurate phylogenetic estimates. Nature Methods 14: 587-589.

Kasahara, S., A.P.Z. Silva, and C.F.B. Haddad. 1996. Chromosome banding in three species of Brazilian toads. Brazilian Journal of Biology 19: 237-242.

Katoh, K., and H. Toh. 2008. Recent developments in the MAFFT multiple sequence alignment program. Briefings in Bioinformatics 9: 286-298.

Katoh, K., J. Rozewicki, and K.D. Yamada. 2019. MAFFT online service: multiple sequence alignment, interactive sequence choice and visualization. Briefings in Bioinformatics 20: 1160-1166.

Kehr, A.I., and J.D. Williams. 1990. Larvas de anuros de la republica Argentina. Cuadernos de Herpetología Serie Monografías 2: 1-44.

Kenny, J.S. 1969. The Amphibia of Trinidad. Studies of the Fauna of Curaçao and Other Caribbean Islands 29: $1-78$.

Klymus, K.E., S.C. Humfeld, V.T. Marshall, D. Cannatella, and H.C. Gerhardt. 2010. Molecular patterns of differentiation in canyon treefrogs ( $\mathrm{Hyla}$ arenicolor): evidence for introgressive hybridization with the Arizona treefrog (H. wrightorum) and correlations with advertisement call differences. Journal of Evolutionary Biology 23: 14251435.
Kluge, A.G. 1989. A concern for evidence and a phylogenetic hypothesis of relationships among Epicrates (Boidae, Serpentes). Systematic Biology 38: $7-25$.

Kluge, A.G. 2004. On total evidence: for the record. Cladistics 20: 205-207.

Kluge, A.G., and T. Grant. 2006. From conviction to anti-superfluity: old and new justifications of parsimony in phylogenetic inference. Cladistics 22: 276288.

Köhler, J. 2000. Amphibian diversity in Bolivia: a study with special reference to montane forest regions. Bonner Zoologische Monographien 48: 1-243.

Köhler, J., A. John, and W. Böhme. 2006. Notes on amphibians recently collected in the Yungas de La Paz region, Bolivia. Salamandra 42: 21-27.

Kok, P.J.R., and M. Kalamandeen. 2008. Introduction to the taxonomy of the amphibians of Kaieteur National Park, Guyana. Abc Taxa 5: 1-278.

Kolenc, F, et al. 2013. The tadpole and karyotype of Rhinella achavali (Anura: Bufonidae). Journal of Herpetology 47: 599-606.

Kutrup, B., U. Bulbul, and N. Yilmaz. 2006. Effects of the ecological conditions on morphological variations of the green toad, Bufo viridis, in Turkey. Ecological Research 21: 208-214.

Kwet, A., M. di Bernardo, and R. Maneyro. 2006. First record of Chaunus achavali (Anura, Bufonidae) from Rio Grande do Sul, Brazil, with a key for the identification of the species in the Chaunus marinus group. Iheringia, Série Zoologia 96: 479-485.

La Marca, E., and A. Mijares-Urrutia. 1996. Taxonomy and geographic distribution of a northwestem Venezuelan toad (Anura, Bufonidae, Bufo sternosignatus). Alytes 14: 101-114.

Lajmanovich, R.C., A.M. Attademo, P.M. Peltzer, C.M. Junges, and M.C. Cabagna. 2011. Toxicity of four herbicide formulations with glyphosate on Rhinella arenarum (Anura: Bufonidae) tadpoles: B-esterases and glutathione S-transferase inhibitors. Archives of Environmental Contamination and Toxicology 60: 681-689.

Lamb, T., and J.C. Avise. 1986. Directional introgression of mitochondrial DNA in a hybrid population of tree frogs: the influence of mating behavior. Proceedings of the National Academy of Sciences 83: 2526-2530.

Landestoy T., M.A., D.B. Turner, A.B. Marion, and S.B. Hedges. 2018. A new species of Caribbean toad (Bufonidae, Peltophryne) from southern Hispaniola. Zootaxa 4403: 523-539. 
Lanfear, R., B. Calcott, S.Y. Ho, and S. Guindon. 2012. PartitionFinder: combined selection of partitioning schemes and substitution models for phylogenetic analyses. Molecular Biology and Evolution 29: 1695-1701.

Larson, P.M. 2004. Chondrocranial morphology and ontogenetic allometry in larval Bufo americanus (Anura, Bufonidae). Zoomorphology 123: 95-106.

Larson, P.M., and R.O. de Sá. 1998. Chondrocranial morphology of Leptodactylus larvae (Leptodactylidae: Leptodactylinae): its utility in phylogenetic reconstruction. Journal of Morphology 238: 287305.

Laurent, R.F. 1969. Las supuestas razas de Bufo arenarum y el concepto de subespecie criptica. Acta Zoológica Lilloana 25: 67-80.

Laurenti, J.N. 1768. Specimen medicum, exhibens synopsis reptilium emendatam, cum experimentis circa venena et antidote reptilium austriacorum, Wien, Austria.

Lavanchy, G., and T. Schwander. 2019. Hybridogenesis. Current Biology 29: R9-R11

Lavilla, E.O., and F. Brusquetti. 2018. On the identity of Bufo diptychus Cope, 1862 (Anura: Bufonidae). Zootaxa 4442: 161-170.

Lavilla, E.O., and J.M. Cei. 2001. Amphibians of Argentina. A second update, 1987-2000. Monografie di Museo Regionale di Scienze Naturali di Torino 18: 1-177.

Lavilla, E.O., and R.O. de Sá. 2001. Chondrocranium and visceral skeleton of Atelopus tricolor and Atelophryniscus chrysophorus tadpoles (Anura, Bufonidae). Amphibia-Reptilia 22: 167-177.

Lavilla, E.O., M.L. Ponssa, and S. Saleme. 2000. Caracterización de las larvas de Bufo fernandezae Gallardo, 1957 y Bufo granulosus major Müller \& Hellmich, 1936 (Anura: Bufonidae) y clave para la identificación de las larvas de Bufo que habitan el Chaco argentino. Bollettino del Museo Regionale di Scienze Naturali 17: 333-344.

Lavilla, E.O., J.S. Barrionuevo, and D. Baldo. 2002. Los anfibios insuficientemente conocidos en Argentina: una reevaluación. Cuadernos de Herpetología 16: 99-118.

Lavilla, E.O., U. Caramaschi, J.A. Langone, J.P. Pombal, and R.O. de Sá. 2013. The identity of Rana margaritifera Laurenti, 1768 (Anura, Bufonidae). Zootaxa 3646: 251-264.

Lavilla, E.O., J.A. Langone, U. Caramaschi, J.P. Pombal, and R.O. de Sá. 2017. Comments on the neotype of Rana margaritifera Laurenti, 1768 (Anura, Bufonidae). Zootaxa 4286: 113-114.
Leão, A.T., and D.M. Cochran. 1952. Revalidation and re-description of Bufo ocellatus Günther, 1858 (Anura: Bufonidae). Memórias do Instituto Butan$\tan 24:$ 271-280.

Lehr, E., G. Köhler, C. Aguilar, and E. Ponce. 2001. New species of Bufo (Anura: Bufonidae) from central Peru. Copeia 2001: 216-223.

Lehr, E., J.B. Pramuk, and M. Lundberg. 2005. A new species of Bufo (Anura: Bufonidae) from andean Peru. Herpetologica 61: 308-318.

Lehr, E., J.B. Pramuk, S.B. Hedges, and J.H. Córdova. 2007. A new species of arboreal Rhinella (Anura: Bufonidae) from Yanachaga-Chemillén National Park in central Peru. Zootaxa 1662: 1-14.

Lehtinen, R.M., M.J. Lannoo, and R.J. Wassersug. 2004. Phytotelm breeding anurans: past, present and future research. Miscellaneous Publications, Museum of Zoology, University of Michigan 193: 1-9.

Lentini, A. 2000. Puerto Rican crested toad (Peltophryne lemur). SSP husbandry manual. Keeper and curator edition. Scarborough, Ontario: Toronto Zoo.

León de Castro, W., and D. Rey Sánchez, D. 2014. Primer registro de la presencia y reproducción del anuro Rhinella poeppigii (Tschudi, 1845) en un área antrópica del Departamento de Lima, Perú. Ecología Aplicada 13: 109-115.

Leviton, A.E., and W.E. Duellman. 1978. A case of homonymy in Bufo (Amphibia, Anura, Bufonidae). Journal of Herpetology 12: 246-247.

Lewis, P.O. 2001. A likelihood approach to estimating phylogeny from discrete morphological character data. Systematic Biology 50: 913-925.

Liedtke, H.C., H. Müller, J. Hafner, P. Nagel, and S. P. Loader. 2014. Interspecific patterns for egg and clutch sizes of African Bufonidae (Amphibia: Anura). Zoologischer Anzeiger 253: 309-315.

Liedtke, H.C., et al. 2016. No ecological opportunity signal on a continental scale? Diversification and life-history evolution of African true toads (Anura: Bufonidae). Evolution 70: 1717-1733.

Liedtke, H.C., et al. 2017. Terrestrial reproduction as an adaptation to steep terrain in African toads. Proceedings of the Royal Society B, Biological Sciences 284: 20162598.

Liem, S.S. 1970. The morphology, systematics and evolution of the Old World treefrogs (Rhacophoridae and Hyperoliidae). Fieldiana (Zoology) 57: 1-145.

Lima, A.P., et al. 2006. Guide to the frogs of Reserva Adolpho Ducke, central Amazonia. Manaus: Áttema Design Editorial. 
Lima, A.P., M. Menin, and M.C. Araújo. 2007. A new species of Rhinella (Anura: Bufonidae) from Brazilian Amazon. Zootaxa 1663: 1-15.

Limeses, C.E. 1964. La musculatura del muslo en los ceratofrínidos y formas afines, con un análisis crítico sobre la significación de los caracteres miologicos en la sistemática de los anuros superiores. Contribuciones Científicas de la Facultad de Ciencias Exactas y Naturales, serie Zoología 1: 193-245.

Limeses, C.E. 1965. La musculatura mandibular en los ceratofrínidos y formas afines (Anura, Ceratophrynidae). Physis 25: 41-58.

Linnaeus, C. 1758. Systema naturae per regna tria naturae, secundum classes, ordines, genera, species, cum characteribus, differentiis, synonymis, locis. 10th ed., vol. 1. Holmiae [Stockholm]: Laurentii Salvii.

Liu, C.C. 1935. Types of vocal sac in the Salientia. Proceedings of the Boston Society of Natural History 41: $19-40$

Liu, K., et al. 2010. Rampant historical mitochondrial genome introgression between two species of green pond frogs, Pelophylax nigromaculatus and P. plancyi. BMC Evolutionary Biology 10: 201.

Lötters, S., and J. Köhler. 2000. A new toad of the Bufo typhonius complex from humid montane forests of Bolivia. Spixiana 23: 293-303.

Lutz, A. 1925. Batraciens du Brésil. Comptes Rendus et Mémoires Hebdomadaires des Séances de la Société de Biologie et des ses Filiales, Paris 93: 211-214.

Lutz, A. 1934. Notas sobre espécies brasileiras do gênero Bufo. Memórias do Instituto Oswaldo Cruz 28: 111-132.

Lynch, J.D. 1973. The transition from archaic to advanced frogs. In J.L. Vial (editor), Evolutionary biology of the anurans: contemporary research on major problems: 131-182. Columbia: University of Missoury Press.

Lynch, J.D. 1978. A re-assessment of the Telmatobiine leptodactylid frogs of Patagonia. Occasional Papers of the Museum of Natural History, University of Kansas 72: 1-57.

Lynch, J.D. 2006. The tadpoles of frogs and toads found in the lowlands of northern Colombia. Revista de la Academia Colombiana de Ciencias Exactas, Físicas y Naturales 30: 443-457.

Lynch, J.D., and J.M. Renjifo. 1990. Two new toads (Bufonidae: Rhamphophryne) from the northern Andes of Colombia. Journal of Herpetology 24: 364-371.

Machado, D.J., M.L. Lyra, and T. Grant. 2016. Mitogenome assembly from genomic multiplex libraries: comparison of strategies and novel mitogenomes for five species of frogs. Molecular Ecology Resources 16: 686-693.

Maciel, N.M., et al. 2006. A phylogenetic analysis of species in the Bufo crucifer group (Anura: Bufonidae), based on indolealkylamines and proteins from skin secretions. Biochemical Systematics and Ecology 34: 457-466.

Maciel, N.M., R.A. Brandão, L.A. Campos, and A. Sebben. 2007. A large new species of Rhinella (Anura: Bufonidae) from Cerrado of Brazil. Zootaxa 1627: 23-39.

Maciel, N.M., R.G. Collevatti, G.R. Colli, and E.F. Schwartz. 2010. Late Miocene diversification and phylogenetic relationships of the huge toads in the Rhinella marina (Linnaeus, 1758) species group (Anura: Bufonidae). Molecular Phylogenetics and Evolution 57: 787-797.

Maddison, W.P. 1993. Missing data versus missing characters in phylogenetic analysis. Systematic Biology 42: 576-581.

Maddison, W.P., and D.R. Maddison. 2018. Mesquite: a modular system for evolutionary analysis. Version 3.51. Online resource (http://mesquiteproject.org).

Mailho-Fontana, P.L., et al. 2018. Morphological and biochemical characterization of the cutaneous poison glands in toads (Rhinella marina group) from different environments. Frontiers in Zoology 15: 46.

Malkmus, R., U. Manthey, G. Vogel, P. Hoffmann, and J. Kosuch. 2002. Amphibians and reptiles of Mount Kinabalu (north Borneo). Ruggell, Liechtenstein: Serpents Tale NHBD/Gantner Verlag Kommanditgesellschaft.

Malmos, K.B., B.K. Sullivan, and T. Lamb. 2001. Calling behavior and directional hybridization between two toads (Bufo microscaphus $\mathrm{x}$ B. woodhousii) in Arizona. Evolution 55: 626-630.

Malone, J.H., and B.E. Fontenot. 2008. Patterns of reproductive isolation in toads. PLoS ONE 3: e3900.

Maneyro, R., and A. Kwet. 2008. Amphibians in the border region between Uruguay and Brazil: Updated species list with comments on taxonomy and natural history (part i: Bufonidae). 1. Stuttgarter Beiträge zur Naturkunde A, Neue Serie 1: 95-121.

Maneyro, R., D. Arrieta, and R.O. de Sá. 2004. A new toad (Anura: Bufonidae) from Uruguay. Journal of Herpetology 38: 161-165.

Markovich, D., and R.R. Regeer. 1999. Expression of membrane transporters in cane toad Bufo marinus oocytes. Journal of Experimental Biology 202 (16): 2217-2223. 
Martin, R.F. 1972a. Osteology and evolution in Neotropical Bufo. American Midland Naturalist 88: 301-317.

Martin, R.F. 1972b. Evidence from osteology. In W.F. Blair (editor), Evolution in the genus Bufo: 37-70. Austin: University of Texas Press.

Martin, R.F. 1973. Osteology of North American Bufo: the americanus, cognatus, and boreas species groups. Herpetologica 29: 375-387.

Martin, W.F. 1972. Evolution of vocalizations in the genus Bufo. In W.F. Blair (editor), Evolution in the genus Bufo: 279-309. Austin: University of Texas Press.

Masta, S.E., B.K. Sullivan, T. Lamb, and E.J. Routman. 2002. Molecular systematics, hybridization, and phylogeography of the Bufo americanus complex in eastern North America. Molecular Phylogenetics and Evolution 24: 302-314.

Matavelli, R., A.M. Campos, G.R. Silva, and G.V. Andrade. 2014. First record of Rhinella ocellata (Günther, 1858) (Bufonidae) for the State of Maranhão, northeastern Brazil. Check List 10: 432-433.

McAlister, W.H. 1961. The mechanics of sound production in North American Bufo. Copeia 1961: 86-95.

McCranie, J.R. 2017. Atelophryniscus, Atelophryniscus chrysophorus. Catalogue of American Amphibians and Reptiles (CAAR) 908: 1-13.

McCranie, J.R., and F.E. Castañeda. 2005. The herpetofauna of Parque Nacional Pico Bonito, Honduras. Phyllomedusa 4: 3-16.

McCranie, J.R., and L.D. Wilson. 2002. The amphibians of Honduras. Ithaca, NY: Society for the Study of Amphibians and Reptiles.

McCranie, J.R., L.D. Wilson, and K.L. William. 1989. A new genus and species of toad (Anura: Bufonidae) with a extraordinary stream-adapted tadpole from northern Honduras. Occasional Papers of the Museum of Natural History, University of Kansas 129: 1-18.

McDade, L.A. 1992. Hybrids and phylogenetic systematics II. The impact of hybrids on cladistic analysis. Evolution 46: 1329-1346.

McDiarmid, R.W. 1971. Comparative morphology and evolution of frogs of the genera Atelopus, Dendrophryniscus, Melanophryniscus, and Oreophrynella. Bulletin of Los Angeles County Museum of Natural History 12: 1-66.

McDiarmid, R.W., and R. Altig. 1999. Research materials and techniques. In R.W. McDiarmid and R. Altig (editors), Tadpoles: the biology of anuran larvae: 7-23. Chicago: University of Chicago Press.
Meier, R., G. Zhang, and F. Ali. 2008. The use of mean instead of smallest interspecific distances exaggerates the size of the "barcoding gap" and leads to misidentification. Systematic Biology 57 : 809-813.

Melin, D. 1941. Contribution to the knowledge of the Amphibia of South America. Göteborgs Kungl Vetenskaps-och Vitterhets-samhälles Handlingar Serien B, Matematiska och Naturvetenskapliga Skrifter 1: 1-71.

Mendelson, J.R. 1997a. Systematics of the Bufo valliceps group (Anura: Bufonidae) of Middle America. Ph.D. dissertation, Department of Systematics and Ecology, University of Kansas, Lawrence.

Mendelson, J.R. 1997b. A new species of Bufo (Anura: Bufonidae) from the Pacific Highlands of Guatemala and southern Mexico, with comments on the status of Bufo valliceps macrocristatus. Herpetologica 53:14-30

Mendelson, J.R., H.R. Silva, and A.M. Maglia. 2000. Phylogenetic relationships among marsupial frog genera (Anura: Hylidae: Hemiphractinae) based on evidence from morphology and natural history. Zoological Journal of the Linnean Society 128: 125148.

Mendelson, J.R., D.G. Mulcahy, T.S. Williams, and J.W. Sites. 2011. A phylogeny and evolutionary natural history of Mesoamerican toads (Anura: Bufonidae: Incilius) based on morphology, life history, and molecular data. Zootaxa 3138: 1-34.

Menin, M., D.J. Rodrigues, and A.P. Lima. 2006. The tadpole of Rhinella proboscidea (Anura: Bufonidae) with notes on adult reproductive behavior. Zootaxa 1258: 47-56.

Mercês, E.A., F. Acuña Juncá, and F.S. Cousiño Casal. 2009. Girinos de três espécies do gênero Rhinella Fitzinger, 1926 (Anura-Bufonidae), ocorrentes no estado da Bahia, Brasil. Sitientibus (Biologia) 9: 133-138.

Mijares-Urrutia, A., and A. Arends. 2001. A new toad of the Bufo margaritifer complex (Amphibia: Bufonidae) from northwestern Venezuela. Herpetologica 57: 523-531.

Milto, K.D., and A.V. Barabanov. 2011. An annotated catalogue of the amphibian types in the collection of the Zoological Institute, Russian Academy of Sciences, St. Petersburg. Russian Journal of Herpetology 18: 137-153.

Minh, B.Q., M.A.T. Nguyen, and A. von Haeseler. 2013. Ultrafast approximation for phylogenetic bootstrap. Molecular Biology and Evolution 30: 1188-1195. 
Miranda, N.E.O., N.M. Maciel, K.P. Tepedino, and A. Sebben 2015. Internal larval characters in anuran systematic studies: a phylogenetic hypothesis for Leptodactylus (Anura, Leptodactylidae). Journal of Zoological Systematics and Evolutionary Research 53: $55-66$.

Molina, G.I. 1782. Saggio sulla Storia Naturale del Chili. Bologna: S. Tommaso d'Aquino.

Moravec, J., E. Lehr, J.C. Cusi, J.H. Córdova, and V. Gvoždík. 2014. A new species of the Rhinella margaritifera species group (Anura: Bufonidae) from the montane forest of the Selva Central, Peru. ZooKeys 371: 35-56.

Morrison, M.E. 1992. A reassessment of the Bufo spinulosus group (Anura: Bufonidae) from Peru and Ecuador. M.Sc. thesis, Department of Systematics and Ecology, University of Kansas, Lawrence.

Morrison, M.E. 1994. A phylogenetic analysis of the Bufo spinulosus group (Anura: Bufonidae). Ph.D. dissertation, Department of Systematics and Ecology, University of Kansas, Lawrence.

Motta, J., M. Menin, A.P. Almeida, T. Hrbek, and I.P. Farias. 2018. When the unknown lives next door: a study of central Amazonian anurofauna. Zootaxa 4438: 79-104.

Mulcahy, D.G., and J.R. Mendelson. 2000. Phylogeography and speciation of the morphologically variable, widespread species Bufo valliceps, based on molecular evidence from mtDNA. Molecular Phylogenetics and Evolution 17: 173-189.

Mulcahy, D.G., B.H. Morrill, and J.R. Mendelson. 2006. Historical biogeography of lowland species of toads (Bufo) across the trans-Mexican neovolcanic belt and the Isthmus of Tehuantepec. Journal of Biogeography 33: 1889-1904.

Müller, L., and W. Hellmich. 1932. Beiträge zur kenntnis der herpetofauna Chiles. I. Über Borborocoetes kriegi und die larven einiger Chilenischer anouren. Zoologischer Anzeiger 97: 204-211.

Müller, L., and W. Hellmich. 1936. Amphibien und Reptilien. I. Teil: Amphibia, Chelonia, Loricata. Wissenschaftliche Ergebnisse der Deutschen Gran Chaco-Expedition. Amphibien und Reptilien: 1-120. Stuttgart: Strecker und Schröder.

Murphy, J.C., T.A. Sierra, J.R. Downie, and M.J. Jowers. 2017. Toads, tall mountains and taxonomy: the Rhinella granulosa group (Amphibia: Anura: Bufonidae) on both sides of the Andes. Salamandra 53: 267-278.

Myers, G.S., and A.L. Carvalho. 1945. Notes on some new or little known Brazilian amphibians, with an examination of the history of the Plata salamander, Ensatina platensis. Boletim do Museu Nacional do Rio de Janeiro 35: 1-24.

Myers, G.S., and A.L. Carvalho. 1952. A new dwarf toad from southeastern Brazil. Zoologica, New York 37: $1-3$.

Narvaes, P., and M.T. Rodrigues. 2009. Taxonomic revision of Rhinella granulosa species group (Amphibia, Anura, Bufonidae), with a description of a new species. Arquivos de Zoologia 40: 1-73.

Nguyen, L.T., H.A. Schmidt, A. Von Haeseler, and B.Q. Minh. 2015. IQ-TREE: a fast and effective stochastic algorithm for estimating maximum-likelihood phylogenies. Molecular Biology and Evolution 32: 268-274.

Nixon, K.C. 1999. The parsimony ratchet, a new method for rapid parsimony analysis. Cladistics 15 : 407-414.

Nixon, K.C., and J.M. Carpenter. 1996. On simultaneous analysis. Cladistics 12: 221-241.

Nixon, K.C., and Q.D. Wheeler. 1992. Extinction and the origin of species. In M.J. Novacek and Q.D. Wheeler (editors), Extinction and phylogeny: 119143. New York: Columbia University Press.

Noble, G.K. 1920. Two new batrachians from Colombia. Bulletin of the American Museum of Natural History 42 (9): 441-446.

Noble, G.K. 1922. The phylogeny of the Salientia. I. The osteology and the thigh musculature; their bearing on classification and phylogeny. Bulletin of the American Museum of Natural History 46 (1): 1-87.

Noble, G.K. 1931. The biology of the Amphibia. New York: McGraw-Hill.

Nokhbatolfoghahai, M., and J.R. Downie. 2005. Larval cement gland of frogs: comparative development and morphology. Journal of Morphology 263: 270283.

Nokhbatolfoghahai, M., and J.R. Downie. 2008. The external gills of anuran amphibians: comparative morphology and ultrastructure. Journal of Morphology 269: 1197-1213.

Noronha, J.C., et al. 2013. Climbing behaviour of terrestrial bufonids in the genus Rhinella. Herpetological Bulletin 124: 22-23.

Nussbaum, R.A., and S.-H. Wu. 2007. Morphological assessments and phylogenetic relationships of the seychellean frogs of the family Sooglossidae (Amphibia: Anura). Zoological Studies 46: 322335.

Obertegger, U., A. Cieplinski, D. Fontaneto, and S. Papakostas. 2018. Mitonuclear discordance as a con- 
founding factor in the DNA taxonomy of monogonont rotifers. Zoologica Scripta 47: 122-132.

O’Donohoe, M.E.A., et al. 2019. Diversity and evolution of the parotoid macrogland in true toads (Anura: Bufonidae). Zoological Journal of the Linnean Society 187: 453-478.

Ohler, A., and A. Dubois. 2006. Phylogenetic relationships and generic taxonomy of the tribe Paini (Amphibia, Anura, Ranidae, Dicroglossinae), with diagnoses of two new genera. Zoosystema 28: 769-784.

Oliveira, R., M.I. Rosa, L.N. Weber, and M.F. Napoli. 2014. Chondrocranial and hyobranchial morphology in larvae of the genus Rhinella Fitzinger, 1826 (Amphibia, Anura, Bufonidae). Herpetological Journal 24: 229-237.

Orozco-terWengel, P., F. Andreone, E. Louis, and M. Vences. 2013. Mitochondrial introgressive hybridization following a demographic expansion in the tomato frogs of Madagascar, genus Dyscophus. Molecular Ecology 22: 6074-6090.

Padial, J.M., S. Reichle, R.W. McDiarmid, and I. De la Riva. 2006. A new species of arboreal toad (Anura: Bufonidae: Chaunus) from Madidi National Park, Bolivia. Zootaxa 1278:57-68.

Padial, J.M., J.C. Chaparro, J. Köhler, and I. De la Riva. 2009. Rediscovery, resurrection and redescription of Rhinella leptoscelis (Boulenger, 1912) (Anura: Bufonidae). Zootaxa 2115: 56-64.

Paluh, D.J., E.L. Stanley, and D.C. Blackburn. 2020. Evolution of hyperossification expands skull diversity in frogs. Proceedings of the National Academy of Sciences of the United States of America 117: 8554-8562.

Palumbi, S.R., A. Martin, W.O. McMillan, L. Stice, and G. Grabowski. 1991. The simple fool's guide to PCR, Version 2.0: privately published document compiled by S. Palumbi.

Parham, J.F, et al. 2012. Best practices for justifying fossil calibrations. Systematic Biology 61: 346-359.

Pauly, G.B., D.M. Hillis, and D.C. Cannatella. 2004. The history of a nearctic colonization: molecular phylogenetics and biogeography of the Nearctic toads (Bufo). Evolution 58: 2517-2535.

Penhos, J.C., B. Uno, and B.A. Houssay. 1967. Glucose and lipid metabolism in the toad's perfused liver. General and Comparative Endocrinology 8: 297304.

Peracca, M.G. 1904. Viaggio del Dr. Enrico Festa nell' Ecuador e regioni vicine. Rettili ed anfibi. Bollettino dei Musei di Zoologia e Anatomia Comparata della R. Universita di Torino 19 (465): 1-41.
Pereyra, M.O., M.F. Vera Candioti, J. Faivovich, and D. Baldo. 2015. Egg clutch structure of Rhinella rumbolli (Anura: Bufonidae), a toad from the Yungas of Argentina, with a review of the reproductive diversity in Rhinella. Salamandra 51: 161-170.

Pereyra, M.O., et al. 2016a. Phylogenetic relationships of toads of the Rhinella granulosa group (Anura: Bufonidae): a molecular perspective with comments on hybridization and introgression. Cladistics 32: 36-53.

Pereyra, M.O., et al. 2016b. The complex evolutionary history of the tympanic middle ear in frogs and toads (Anura). Scientific Reports 6: 34130.

Pérez-Ben, C.M., G.O. Gómez, and A.M. Báez. 2014. Intraspecific morphological variation and its implications in the taxonomic status of 'Bufo pisanoi,' a Pliocene anuran from eastern Argentina. Journal of Vertebrate Paleontology 34: 767-773.

Pérez-Ben, C.M., G.O. Gómez, and A.M. Báez. 2019. A new Pliocene true toad (Anura: Bufonidae): first record of an extinct species from South America. Journal of Vertebrate Paleontology 39: e1576183

Perotti, M.G. 1994. Aportes preliminares sobre la reproducción en una comunidad de anuros chaqueños en Argentina. Cuadernos de Herpetología 8: 39-50.

Philippi, R.A. 1902. Suplemento a los batraquios chilenos descritos en la Historia Física i Política de Chile de don Claudio Gay. Santiago de Chile: Libreria Alemana de Jose Ivens.

Pimentel, R.A., and R. Riggins. 1987. The nature of cladistic data. Cladistics 3: 201-209.

Plytycz, B., and H. Szarski. 1987. Inguinal bodies of some Bufo species. Journal of Herpetology 21: 236-237.

Pol, D., and S. Apesteguía. 2005. New Araripesuchus remains from the early late Cretaceous (Cenomanian-Turonian) of Patagonia. American Museum Novitates 3490: 1-38.

Ponssa, M.L. 2008. Cladistic analysis and osteological descriptions of the frog species in the Leptodactylus fuscus species group (Anura, Leptodactylidae). Journal of Zoological Systematics and Evolutionary Research 46: 249-266.

Portik, D.M., and T.J. Papenfuss. 2015. Historical biogeography resolves the origins of endemic Arabian toad lineages (Anura: Bufonidae): evidence for ancient vicariance and dispersal events with the horn of Africa and south Asia. BMC Evolutionary Biology 15: 152.

Posada, D., and K.A. Crandall. 2002. The effect of recombination on the accuracy of phylogeny estimation. Journal of Molecular Evolution 54 : 396402 . 
Power, J. 1925. Notes on the habits and life-histories of certain little-known Anura, with descriptions of the tadpoles. Transactions of the Royal Society of South Africa 13: 107-117.

Poynton, J.C. 1964. Amphibia of the Nyasa-Luangwa region of Africa. Senckenbergiana Biologica 45: 193-225.

Pramuk, J.B. 2006. Phylogeny of South American Bufo (Anura: Bufonidae) inferred from combined evidence. Zoological Journal of the Linnean Society 146: 407-452.

Pramuk, J.B., and E. Lehr. 2005. Taxonomic status of Atelophryniscus chrysophorus McCranie, Wilson, and Williams, 1989 (Anura: Bufonidae) inferred from phylogeny. Journal of Herpetology 39: 610-618.

Pramuk, J.B., and F. Kadivar. 2003. A new species of Bufo (Anura: Bufonidae) from southern Ecuador. Herpetologica 59: 270-283.

Pramuk, J.B., C.A. Hass, and S.B. Hedges. 2001. Molecular phylogeny and biogeography of West Indian toads (Anura: Bufonidae). Molecular Phylogenetics and Evolution 20: 294-301.

Pramuk, J.B., T. Robertson, J.W. Sites, and B.P. Noonan. 2008. Around the world in 10 million years: biogeography of the nearly cosmopolitan true toads (Anura: Bufonidae). Global Ecology and Biogeography 17: 72-83.

Pugener, L.A., A.M. Maglia, and L. Trueb. 2003. Revisiting the contribution of larval characters to an analysis of phylogenetic relationships of basal anurans. Zoological Journal of the Linnean Society 139: 129-155.

Pyron, R.A. 2014. Biogeographic analysis reveals ancient continental vicariance and recent oceanic dispersal in amphibians. Systematic Biology 63: 779-797

Pyron, R.A., and J.J. Wiens. 2011. A large-scale phylogeny of Amphibia including over 2800 species, and a revised classification of extant frogs, salamanders, and caecilians. Molecular Phylogenetics and Evolution 61: 543-583.

Rada de Martínez, D. 1990. Contribución al conocimiento de las larvas de anfibios de Venezuela. Memoria de la Sociedad de Ciencias Naturales La Salle 49-50: 391-403.

Rambaut, A. 2016. FigTree, tree figure drawing tool, Version 1.4.3. Online resource (http://tree.bio.ed. ac.uk/software/figtree).

Rash, L.D., R.A. Morales, S. Vink, and P.F. Alewood. 2011. De novo sequencing of peptides from the parotid secretion of the cane toad, Bufo marinus (Rhinella marina). Toxicon 57: 208-216.
Rivero, J.A., and C.J. Castaño. 1990. A new and peculiar species of Rhamphophryne (Amphibia: Bufonidae) from Antioquia, Colombia. Journal of Herpetology 24: $1-5$.

Rivero, J.A., H. Mayorga, E. Estremera, and I. Izquierdo. 1980. Sobre el Bufo lemur (Cope) (Amphibia, Bufonidae). Caribbean Journal of Science 15: 33-40.

Roberto, I.J., L. Brito, and P. Cascon. 2011. Temporal and spatial patterns of reproductive activity in $R h i-$ nella hoogmoedi (Anura: Bufonidae) from a tropical rainforest in northeastern Brazil, with the description of its advertisement call. South American Journal of Herpetology 6: 87-98.

Roberto, I.J., L. Brito, and M.T.C. Thomé. 2014. A new species of Rhinella (Anura: Bufonidae) from northeastern Brazil. South American Journal of Herpetology 9: 190-199.

Rodríguez, C., L. Rollins-Smith, R. Ibáñez, A.A. Durant-Archibold, and M. Gutiérrez. 2017. Toxins and pharmacologically active compounds from species of the family Bufonidae (Amphibia, Anura). Journal of Ethnopharmacology 198: 235-254.

Rodríguez, L.O., and W.E. Duellman. 1994. Guide to the frogs of the Iquitos region, Amazonian Peru. University of Kansas Natural History Museum Special Publication 22: 1-80.

Rodríguez, L.O., J. H. Córdova, and J. Icochea. 1993. Lista preliminar de los anfibios del Perú. Publicaciones del Museo de Historia Natural, Universidad Nacional Mayor de San Marcos (ser. A) 45: 1-22

Roelants, K., and F. Bossuyt. 2005. Archaeobatrachian paraphyly and Pangaean diversification of crowngroup frogs. Systematic Biology 54: 111-126.

Roelants, K., et al. 2007. Global patterns of diversification in the history of modern amphibians. Proceedings of the National Academy of Sciences of the United States of America 104: 887-892.

Rojas-Runjaic, F.J.M., E. Camargo, V. Carvalho, and E. La Marca. 2017. New record and range extension of the horned toad, Rhinella ceratophrys (Boulenger, 1882) (Anura: Bufonidae), in Venezuela, and confirmation of its presence in Brazil. Check List 13: 2035.

Rollins, L.A., M. F. Richardson, and R. Shine. 2015. A genetic perspective on rapid evolution in cane toads (Rhinella marina). Molecular Ecology 24: 22642276.

Romero, J.H., C.C. Vidal, and J.D. Lynch. 2008. Estudio preliminar de la fauna Amphibia en el cerro Murrucucú, Parque Natural Nacional Paramillo y zona amortiguadora, Tierralta, Córdoba, Colombia. Caldasia 30: 209-229. 
Ron, S.R., et al. 2015. Systematics of the endangered toad genus Andinophryne (Anura: Bufonidae): phylogenetic position and synonymy under the genus Rhaebo. Zootaxa 3947: 347-366.

Rose, W. 1962. The Reptiles and Amphibians of Southern Africa. Cape Town, South Africa: Maskew Miller.

Rossa-Feres, D.C., and F. Nomura. 2006. Characterization and taxonomic key for tadpoles (Amphibia: Anura) from the northwestern region of São Paulo State, Brazil. Biota Neotropica 6.

Ruas, D.S., C.V.M. Mendes, B.B. Szpeiter, and M. Solé. 2012. The tadpole of Rhinella crucifer (Wied-Neuwied, 1821) (Amphibia: Anura: Bufonidae) from southern Bahia, Brazil. Zootaxa 3299: 66-68.

Rueda-Almonacid, J.V., J.D. Lynch, and A. Amézquita. 2004. Libro Rojo de los Anfibios de Colombia: Conservación Internacional Colombia, Instituto de Ciencias Naturales-Universidad Nacional de Colombia, Ministerio de Medio Ambiente, Bogotá, Colombia.

Ruiz-Carranza, P.M., M.C. Ardila-Robayo, and J.D. Lynch. 1996. Lista actualizada de la fauna de Amphibia de Colombia. Revista de la Academia Colombiana de Ciencias Exactas, Físicas y Naturales 20: 365-415.

Saito, E.N., T.S. Kunz, and A. Ambrozio-Assis. 2016. First record of Rhinella achavali (Maneyro, Arrieta \& de Sá, 2004) in the state of Santa Catarina, southern Brazil (Anura: Bufonidae). Check List 12: 1-4.

Salthe, S.N. 1963. The egg capsules in the Amphibia. Journal of Morphology 113: 161-171.

Sanabria, E., L. Quiroga, F. Arias, and R. Cortez. 2010. A new species of Rhinella (Anura: Bufonidae) from Ischigualasto Provincial Park, San Juan, Argentina. Zootaxa 2396: 50-60.

Santana, D.J., R. Gaiga, L.F. Storti, T.G. Santos, and M. Dixo. 2010. New state record and distribution map of Rhinella cerradensis (Anura, Bufonidae) in the Cerrado biome, Brazil. Herpetology Notes 3: 55-56.

Santos, J.C., and D.C. Cannatella. 2011. Phenotypic integration emerges from aposematism and scale in poison frogs. Proceedings of the National Academy of Sciences of the United States of America 108: 6175-6180.

Santos, S., R. Ibáñez, and S. Ron. 2015. Systematics of the Rhinella margaritifera complex (Anura, Bufonidae) from western Ecuador and Panama with insights in the biogeography of Rhinella alata. ZooKeys 501: 109-145.

Sassone, A.G., E. Regueira, M.F. Scaia, M.C. Volonteri, and N.R. Ceballos. 2015. Development and ste- roidogenic properties of the Bidder's organ of the tadpole of Rhinella arenarum (Amphibia, Anura). Journal of Experimental Zoology Part A: Ecological Genetics and Physiology 323: 137-145.

Savage, J.M. 2002. The amphibians and reptiles of Costa Rica. Chicago: University of Chicago Press.

Schmid, M. 1978. Chromosome banding in Amphibia. I. Constitutive heterochromatin and nucleolus organizer regions in Bufo and Hyla. Chromosoma 66: 361-388.

Schmid, M., C. Steinlein, and T. Haaf. 2004. Chromosome banding in Amphibia. XXX. Karyotype aberrations in cultured fibroblast cells. Cytogenetic and Genome Research 104: 277-282.

Schmidt, O. 1857. Diagnosen neuer Frösche des zoologischen Cabinets zu Krakau. Sitzungsberichte der Kaiserlichen Akademie der Wissenschaften, Mathematisch-Naturwissenschaftliche Classe 24: 10-15.

Schwarzer, J., et al. 2012. Repeated trans-watershed hybridization among haplochromine cichlids (Cichlidae) was triggered by Neogene landscape evolution. Proceedings of the Royal Society B: Biological Sciences 279: 4389-4398.

Schwenk, K., N. Brede, and B. Streit. 2008. Extent, processes and evolutionary impact of interspecific hybridization in animals. Philosophical Transactions of the Royal Society of London B 363: 2805-2811.

Scott, E. 2005. A phylogeny of ranid frogs (Anura: Ranoidea: Ranidae), based on a simultaneous analysis of morphological and molecular data. Cladistics 21: $507-574$.

Seba, A. 1734. Locupletissimi rerum naturalium thesauri accurata descriptio, et iconibus artificiosissimis expressio, per universam physices historium, opus, cui in hoc rerum genere, nullum par exstitit. Vol. 1. Amsterdam: Janssonio-Waesbergios.

Sedra, S.N. 1950. The metamorphosis of the jaws and their muscles in the toad, Bufo regularis Reuss, correlated with the changes in the animal's feeding habits. Proceedings of the Zoological Society of London 120: 405-448.

Sedra, S.N., and M.I. Michael. 1958. The metamorphosis and growth of the hyobranchial apparatus of the Egyptian toad, Bufo regularis Reuss. Journal of Morphology 103: 1-30.

Sequeira, F., et al. 2011. Hybridization and massive mtDNA unidirectional introgression between the closely related Neotropical toads Rhinella marina and $R$. schneideri inferred from mtDNA and nuclear markers. BMC Evolutionary Biology 11: 264. 
Servedio, M.R., and J. Hermisson. 2019. The evolution of partial reproductive isolation as an adaptive optimum. Evolution 74: 4-14.

Silva, H.R., and J.R. Mendelson. 1999. A new organ and sternal morphology in toads (Anura: Bufonidae): descriptions, taxonomic distribution, and evolution. Herpetologica 55: 114-126.

Silva, L.A., S.P. Dantas, D.L. Santos, H.B. Neto, and D.J. Santana. 2018. Newly distribution of Rhinella gildae Vaz-Silva et al., 2015 (Anura, Bufonidae): a little known species of the Rhinella margaritifera species group. Herpetology Notes 11: 121-125.

Silva, M.I., L.C. Schiesari, and M. Menin. 2017. The egg clutch and tadpole of Rhinella merianae (Gallardo, 1965) (Anura: Bufonidae) from central Amazonia, Brazil. Zootaxa 4294: 145-150.

Simon, M.N., and G. Marroig. 2015. Landmark precision and reliability and accuracy of linear distances estimated by using 3D computed micro-tomography and the open-source tina manual landmarking tool software. Frontiers in Zoology 12: 12.

Simon, M.N., F.A. Machado, and G. Marroig. 2016. High evolutionary constraints limited adaptive responses to past climate changes in toad skulls. Proceedings of the Royal Society B, Biological Sciences 283: 20161783.

Sinsch, U. 1986. Anfibios de la Sierra Central del Perú - una clave de identificacion para adultos y larvas. Boletín de Lima 45: 23-33

Sinsch, U., I.E. di Tada, and A.L. Martino. 2001. Longevity, demography and sex-specific growth of the Pampa de Achala toad, Bufo achalensis Cei, 1972. Studies on Neotropical Fauna and Environment 36: 95-104.

Smith, S.A., P.R. Stephens, and J.J. Wiens. 2005. Replicate patterns of species richness, historical biogeography, and phylogeny in Holartic treefrogs. Evolution 59: 2433-2450.

Spix, J.B. 1824. Animalia nova sive Species novae Testudinum et Ranarum quas in itinere per Brasiliam annis MDCCCXVII-MDCCCXX jussu et auspiciis Maximiliani Josephi I. Bavariae Regis. München: F.S. Hübschmann.

Stejneger, L. 1913. Results of the Yale Peruvian expedition of 1911. Batrachians and reptiles. Proceedings of the United States National Museum 15: 541547.

Stevaux, M.N. 2002. A new species of Bufo Laurenti (Anura, Bufonidae) from northeastern Brazil. Revista Brasileira de Zoologia 19: 235-242.

Stewart, M.M. 1967. Amphibians of Malawi. Albany: State University of New York.
Stohler, M.R. 1932. Sur la presence de l'ovaire potentiel (organe de Bidder) chez les Bufonidae. Bulletin du Muséum National d'Histoire Naturelle 4: 641-643.

Strong, E.E., and D. Lipscomb. 1999. Character coding and inapplicable data. Cladistics 15: 363-371.

Stynoski, J.L., F.A. Trama, F.L.R. Patrón, E. Tapia, and K.L. Hoke. 2020. Reproductive ecology of the Peruvian earless toad Rhinella yunga (Amphibia, Bufonidae) with descriptions of calls, tadpole, and female competition. South American Journal of Herpetology 15: 85-96.

Sugai, J.L.M.M., F.L. Souza, P. Landgref-Filho, and E. A. Sczesny-Moraes. 2014. Rhinella scitula (Caramaschi \& Niemeyer, 2003) (Amphibia: Anura: Bufonidae): new distribution records. Check List 10: 694-696.

Swofford, D.L. 2002. PAUP* ${ }^{\star}$ Phylogenetic analysis using parsimony ( ${ }^{*}$ and other methods).

Sympson, V., F.G. Jara, and C.A. Úbeda. 2006. Bufo spinulosus papillosus (NCN), reproduction. Herpetological Review 37: 200-201.

Thomé, M.T.C., et al. 2010. Phylogeography of endemic toads and Post-Pliocene persistence of the Brazilian Atlantic Forest. Molecular Phylogenetics and Evolution 55: 1018-1031.

Thomé, M.T.C., K.R. Zamudio, C.F.B. Haddad, and J. Alexandrino. 2012. Delimiting genetic units in Neotropical toads under incomplete lineage sorting and hybridization. BMC Evolutionary Biology 12: 242.

Thominot, A. 1884. Note sur un batracien d'espèce nouvelle provenant de Panama. Bulletin de la Société Philomathique de Paris. Series 7, 8: 151-152.

Tihen, J.A. 1962. Osteological observations on new world Bufo. American Midland Naturalist 67: 157-183.

Titus, T.A., and A. Larson. 1996. Molecular phylogenetics of desmognathine salamanders (Caudata: Plethodontidae): a reevaluation of evolution in ecology, life history, and morphology. Systematic Biology 45: 451-472.

Toews, D.P.L., and A. Brelsford. 2012. The biogeography of mitochondrial and nuclear discordance in animals. Molecular Ecology 21: 3907-3930.

Toledo, R.C., and C. Jared. 1993. Cutaneous adaptations to water balance in amphibians. Comparative Biochemistry and Physiology Part A: Physiology 105: 593-608.

Tolledo, J., and L.F. Toledo. 2010. Tadpole of Rhinella jimi (Anura: Bufonidae) with comments on the tadpoles of species of the Rhinella marina group. Journal of Herpetology 44: 480-483.

Trueb, L. 1970. Evolutionary relationships of casqueheaded tree frogs with co-ossified skulls (family 
Hylidae). University of Kansas Publications, Museum of Natural History 18: 547-716.

Trueb, L. 1971. Phylogenetic relationships of certain Neotropical toads with the description of a new genus (Anura: Bufonidae). Bulletin of Los Angeles County Museum of Natural History 216: 1-40.

Trueb, L. 1973. Bones, frogs, and evolution. In J.L. Vial (editor), Evolutionary biology of the anurans: contemporary research on major problems: 65-132. Columbia: University of Missouri Press.

Trueb, L. 1993. Patterns of cranial diversity among the Lissamphibia. In J. Hanken and B.K. Hall (editors), Patterns of structural and systematic diversity: 255343. Chicago: University of Chicago Press.

Tschudi, J.J. 1845. Reptilium conspectus quae in Republica Peruana reperiuntur et pleraquae observata vel collecta sunt in itinere a Dr. J. J. de Tschudi. Archiv für Naturgeschichte, Berlin 11: 150-170.

Tyler, M.J. 1971. The phylogenetic significance of vocal sac structure in hylid frogs. Miscellaneous Publication University of Kansas Museum of Natural History 19: 319-360.

Urra, F. 2013. Síntesis del conocimiento actual sobre los sapos Rhinella atacamensis, $R$. arunco y R. spinulosa. La Chiricoca 16: 4-15.

Valencia-Zuleta, A., et al. 2020. Vocalizations of Rhinella sebbeni Vaz-Silva, Maciel, Bastos, and Pombal, 2015 (Anura: Bufonidae). Bioacoutics 29: 197-209.

Vallinoto, M., et al. 2010. Phylogeny and biogeography of the Rhinella marina species complex (Amphibia, Bufonidae) revisited: implications for Neotropical diversification hypotheses. Zoologica Scripta 39: 128-140.

Vallinoto, M., D.B. Cunha, A. Bessa-Silva, D. Sodré, and F. Sequeira. 2017. Deep divergence and hybridization among sympatric Neotropical toads. Zoological Journal of the Linnean Society 180: 647-660.

van Bocxlaer, I., S.D. Biju, S.P. Loader, and F. Bossuyt. 2009. Toad radiation reveals into-India dispersal as a source of endemism in the western Ghats-Sri Lanka biodiversity hotspot. BMC Evolutionary Biology 9: 131.

van Bocxlaer, I., et al. 2010. Gradual adaptation toward a range-expansion phenotype initiated the global radiation of toads. Science 327: 679-682.

van Kampen, P.N. 1923. The Amphibia of the IndoAustralian archipelago. Leiden: Brill.

Vanzolini, P.E. 1981. The scientific and political contexts of the Bavarian Expedition to Brasil. In J.B. Spix and J.G. Wagler, Herpetology of Brazil. Ithaca, NY: Society for the Study of Amphibians and Reptiles.
Vaz-Silva, W., P.H. Valdujo, and J.P. Pombal. 2012. New species of the Rhinella crucifer group (Anura, Bufonidae) from the Brazilian Cerrado. Zootaxa 3265: 57-65.

Vaz-Silva, W., N.M. Maciel, R.P. Bastos, and J.P. Pombal. 2015. Revealing two new species of the Rhinella margaritifera species group (Anura, Bufonidae): an enigmatic taxonomic group of Neotropical toads. Herpetologica 71: 212-222.

Vélez-Rodríguez, C.M. 1999. Presencia de Bufo sternosignatus Günther 1859 (Amphibia: Bufonidae) en Colombia. Revista de la Academia Colombiana de Ciencias Exactas, Físicas y Naturales 23 (Supl.): 411-416.

Vélez-Rodríguez, C.M. 2004a. Sapo picudo de Trueb. Rhamphophryne truebae. In J.V. Rueda-Almonacid, J.D. Lynch, and A. Amézquita (editors), Libro rojo de los anfibios de Colombia: 292-295. Bogotá: Conservación Internacional Colombia, Instituto de Ciencias Naturales-Universidad Nacional de Colombia, Ministerio de Medio Ambiente.

Vélez-Rodríguez, C.M. 2004b. Sistemática de los sapos neotropicales pertenecientes al grupo Bufo typhonius (Amphibia: Bufonidae). Ph.D. dissertation, Facultad de Ciencias, Universidad Austral de Chile, Valdivia.

Vélez-Rodriguez, C.M. 2005. Osteology of Bufo sternosignatus Günther, 1858 (Anura: Bufonidae) with comments on phylogenetic implications. Journal of Herpetology 39: 299-303.

Vélez-Rodríguez, C.M., and P.M. Ruiz-Carranza. 2002. A new species of Bufo (Anura: Bufonidae) from Colombia. Herpetologica 58: 453-462.

Vellard, J. 1959. Estudios sobre batracios andinos. V. El género Bufo. Memorias del Museo de Historia Natural "Javier Prado" 8: 1-48.

Venâncio, N.M., M.A. Freitas, A.D. Abegg, and M.N.C. Kokubum. 2017. First record of Rhinella poeppigii (Tschudi, 1845) in Brazil (Anura, Bufonidae). Check List 13: 747-750.

Vences, M., M. Thomas, A. van der Meijden, Y. Chiari, and D.R. Vieites. 2005a. Comparative performance of the 16s rRNA gene in DNA barcoding of amphibians. Frontiers in Zoology 2: 5.

Vences, M., M. Thomas, R.M. Bonett, and D.R. Vieites. 2005b. Deciphering amphibian diversity through DNA barcoding: chances and challenges. Philosophical Transactions of the Royal Society B, Biological Sciences 360: 1859-1868.

Vera Candioti, M.F. 2007. Anatomy of anuran tadpoles from lentic water bodies: systematic relevance and correlation with feeding habits. Zootaxa 1600: $1-175$. 
Vera Candioti, F., et al. 2016. Structural and heterochronic variations during the early ontogeny in toads (Anura: Bufonidae). Herpetological Monographs 30: $79-118$

Vera Candioti, F., et al. 2020. Larval Anatomy of Andean Toads of the Rhinella spinulosa Group (Anura: Bufonidae). Herpetological Monographs 34: $116-130$.

Viertel, B., and A. Channing. 2017. The larva of Schismaderma carens (Smith, 1849) (Anura: Bufonidae): a redescription. Alytes 33: 38-46.

Wassersug, R.J. 1976. A procedure for differential staining of cartilage and bone in whole formalin-fixed vertebrates. Stain Technology 51: 131-134.

Wells, K.D. 2007. The ecology and behavior of amphibians. Chicago: University of Chicago Press.

Werner, F. 1901. Reptilien und Batrachier aus Peru und Bolivien. Abhandlungen und Berichte des Zoologischen und Anthropologisch-Ethnographischen Museums zu Dresden 9: 1-14.

Wheeler, W.C., et al. 2006. Dynamic homology and phylogenetic systematics: a unified approach using POY. New York: American Museum of Natural History.

Wied, M.A.P. 1821. Reise nach Brasilien in den Jahren 1815 bis 1817 . Vol. 2. Franfurt a. M.: Henrich Ludwig Brönner.

Wiegmann, A.F.A. 1833. Herpetologischen Beyträge. I. Ueber die mexicanischen Kröten nebst bemerkungen über ihren verwandte Arten anderer Weltgegenden. Isis von Oken 26: columns 651-662.

Wiegmann, A.F.A. 1834. Amphibien. In F.J.F. Meyen (editor), Reise um die Erde ausfeführt auf dem Königlich Preussischen Seehandlungs-Schiffe Prinzes Louise, comandiert von Captain W. Wendt, in den Jahren 1830, 1831 und 1832 von Dr. F. J. F. Meyen. Dritter Theil. Zoologisher Bericht: 433-522. Berlin: Sander'schen Buchhandlung (C. W. Eichhoff).

Wiens, J.J., J.W. Fetzner, C.L. Parkinson, and T.W. Reeder. 2005. Hylid frog phylogeny and sampling strategies for speciose clades. Systematic Biology 54: 719-748.

Wiley, E.O., G.D. Johnson, and W.W. Dimmick. 1998. The phylogenetic relationships of lampridiform fishes (Teleostei: Acanthomorpha), based on a totalevidence analysis of morphological and molecular data. Molecular Phylogenetics and Evolution 10: 417-425.

Wilkinson, M. 1996. Majority rule reduced consensus and their use in bootstrapping. Molecular Biology and Evolution 13: 437-444.
Wilkinson, J.A., M. Matsui, and T. Terachi. 1996. Geographic variation in a Japanese tree frog (Rhacophorus arboreus) revealed by PCR-aided restriction site analysis of mtDNA. Journal of Herpetology 30 : 418-423.

Will, K.W., and D. Rubinoff. 2004. Myth of the molecule: DNA barcodes for species cannot replace morphology for identification and classification. Cladistics 20: 47-55.

Winokur, R.M., and S. Hillyard. 1992. Pelvic cutaneous musculature in toads of the genus Bufo. Copeia 1992: 760-769.

Wright, A.H., and A.A. Wright. 1949. Handbook of frogs and toads of the United States and Canada. Ithaca, New York: Comstock.

Yamazaki, Y., S. Kouketsu, T. Fukuda, Y. Araki, and H. Nambu. 2008. Natural hybridization and directional introgression of two species of Japanese toads Bufo japonicus formosus and Bufo torrenticola (Anura: Bufonidae) resulting from changes in their spawning habitat. Journal of Herpetology 42: 427-436.

Yanosky, A.A., J.A. Dixon, and C. Mercolli. 1997. Field ecology of the pygmy toad Bufo pygmeus (Anura: Bufonidae), in northeastern Argentina with notes on sympatric sibling species of the granulosus group. Bulletin of the Maryland Herpetological Society 33: 66-77.

Zhang, Q.P., et al. 2018. Interspecies introgressive hybridization in spiny frogs Quasipaa (Family Dicroglossidae) revealed by analyses on multiple mitochondrial and nuclear genes. Ecology and Evolution 8: 1260-1270.

Zhang, D., et al. 2019. "Ghost introgression" as a cause of deep mitochondrial divergence in a bird species complex. Molecular Biology and Evolution 36: 2375-2386.

Zhou, W.W., et al. 2012. Speciation in the Rana chensinensis species complex and its relationship to the uplift of the Qinghai-Tibetan Plateau. Molecular Ecology 21: 960-973. 


\section{APPENDIX 1}

\section{Locality Data of Voucher Specimens and Sources of the Sequences}

Collection abbreviations are as follow: AG, Anna Goebel field series; AACRG, African Amphibian Conservation Research Group, North-West University, Potchefstroom, South Africa; AF, Antoine Fouquet field series; AJC, Andrew J. Crawford field series; AML, Alejandro Montoya L. field series; AMNH, American Museum of Natural History, New York; AMNH-FS, American Museum of Natural History field series, New York; ANDES, Museo de Historia Natural Andes, Universidad de los Andes, Bogotá, Colombia; APL, Albertina P. Lima field series; AR, Alexander Robertson field series; BB, Boris Blotto field series; to be accessioned in MACN; BM, Michel Blanc field series; BMNH, Natural History Museum, London, UK; CAS, California Academy of Sciences, San Francisco, California; CBA, César Barrio-Amoros field series; CFBH, Collection Célio F.B. Haddad, Universidade Estadual Paulista, Rio Claro, São Paulo, Brazil; CFBH-T, Célio F.B. Haddad tissue collection, Universidade Estadual Paulista, Rio Claro, São Paulo, Brazil; CH, Círculo Herpetológico de Panamá, Panamá, Panama; CHUNB, Coleção Herpetológica da Universidade de Brasília, Brasília, Brazil; CORBIDI, Centro de Ornitología y Biodiversidad, Lima, Peru; CTGA-UFAM, tissues collection of Universidade Federal do Amazonas, Manaus, Amazonas, Brazil; CZUT, Colección Zoológica, Facultad de Ciencias, Universidad del Tolima, Tolima, Colombia; DCC, David C. Cannatella field series; DPL, Dwight P. Lawson field series; ESTR, locality code; (Miguel Trefaut Rodrigues field series) FML, Fundación Miguel Lillo, San Miguel de Tucumán, Argentina; FMNH, Field Museum, Chicago, IL; ICN, Universidad Nacional de Colombia, Instituto de Ciencias Naturales, Museo de Historia Natural, Bogotá, Colombia; IDLR, Ignacio de la Riva field series; IIBP, Instituto de Investigación Biológica del Paraguay, Asunción; IWU, Illinois Wesleyan University, Bloomington, IN; IZUA, Instituto de Zoología, Facultad de Ciencias, Universidad Austral de Chile, Valdivia, Chile; JMP, José M. Padial field series; KMH, Kim M. Howell field series; KRL, Karen R. Lips field series; KU, University of Kansas Natural History Museum, Lawrence, Kansas, KS; LAJ, locality code; LGE, Laboratorio de Genética Evolutiva, Universidad Nacional de Misiones, Argentina; LSUMZ, Louisiana State University, Museum of Natural Science, Baton Rouge, Louisiana, LA; MACN, Museo Argentino de Ciencias Naturales "Bernardino Rivadavia"CONICET, Buenos Aires, Argentina; MAR, Marco A. Rada field series; MC, Christian Marty field series; MCZ, Museum of Comparative Zoology, Harvard University, Cambridge, Massachusetts, MA; MHNLS, Museo de Historia Natural La Salle, Fundación La Salle de Ciencias Naturales, Caracas, Venezuela; MHUA, Museo de Herpetología de la Universidad de Antioquia, Medellín, Colombia; MJH, Martin J. Henzl field series; MNCN, Museo Nacional de Ciencias Naturales, Madrid, Spain; MNCN-ADN, Museo Nacional de Ciencias Naturales tissue collection, Madrid, Spain; MNHN-Fr, Muséum national d'Histoire naturelle, Paris, France; MNHN-Uy, Museo Nacional de Historia Natural, Montevideo, Uruguay; MNRJ, Museu Nacional do Rio de Janeiro, Rio de Janeiro, Brazil; MTD, Senckenberg Naturhistorische Sammlungen Dresden, Dresden, Germany; MTR, Miguel Trefaut Rodrigues field series; MTSN, Museo Tridentino di Scienze Naturali, Trento, Italy; MUBI, Museo de Biodiversidad del Perú, Cusco, Peru; MUSM, Museo de Historia Natural, Universidad Nacional Mayor de San Marcos, Lima, Peru; MVUP, Museo de Vertebrados, Universidad de Panamá, Panamá, Panama; MVZ, University of California, Museum of Vertebrate Zoology, Berkeley, CA; MW, Mark Wilkinson field series; MZUSP, Museu de Zoologia, Universidade de São Paulo, São Paulo, Brazil; NB, Néstor Basso field series; MNK, Museo de Historia Natural "Noel Kempff Mercado", Santa Cruz de la Sierra, Bolivia; NMP, Národní muzeum National Museum, Prague, Czech Republic; NP, Nikolai Poyarkov field series; 
PD, Pedro Dias field series; PG, Philippe Gaucher field series; QCAZ, Museo de Zoología, Pontificia Universidad Católica del Ecuador, Quito, Pichincha, Ecuador; RGP, Roberto Gutierrez Poblete field series; ROM, Royal Ontario Museum, Department of Natural History, Toronto, Canada; SBH, S. Blair Hedges field series; SMF, Senckenberg Forschungsinstitut und Naturmuseum, Frankfurt am Main, Germany; TG, Taran Grant field series; TWR, Tod W. Reeder field series; UFMT, Universidade Federal de Mato Grosso, Cuiabá, Mato Grosso, Brazil; UFRGS, Universidade Federal do Rio Grande do Sul, Departamento de Zoologia, Rio Grande do Sul, Brazil; UNSJ, Universidad Nacional de San Juan, San Juan, Argentina; URCA, Universidade Regional do Cariri, Crato, Ceará, Brazil; USNM, National Museum of Natural History, Smithsonian Institution, Washington DC; UTA, University of Texas at Arlington Department of Biology, Arlington, TX; UWIZM, University of the West Indies, Zoology Museum, Department of Life Sciences, St. Augustine, Saint George, Trinidad and Tobago; VG, Václav Gvoždík field series; VUB, Vrije Universiteit Brussel, Belgium; ZUEC, Museu de História Natural, Universidade Estadual de Campinas, Campinas, Brazil; ZUFG, Universidade Federal de Goiás, Goiânia, state of Goiás, Brazil; and ZVC, Colección de Zoología Vertebrados de la Facultad de Ciencias, Montevideo, Uruguay. Abbreviations: nd, no data; nv, no voucher specimen; -, no change in the taxonomy of the species.

\begin{tabular}{|c|c|c|c|c|}
\hline \multicolumn{5}{|l|}{ RHINELLA } \\
\hline Current taxonomy & Updated taxonomy & Voucher & Locality & Sources \\
\hline \multirow{3}{*}{ R. $a b e i$} & \multirow{3}{*}{ R. ornata } & CFBH 18141 & $\begin{array}{l}\text { Brazil: Paraná: Quatro } \\
\text { Barras }\end{array}$ & $\begin{array}{l}\text { This study; Thomé et } \\
\text { al., } 2010\end{array}$ \\
\hline & & MACN 46672 & $\begin{array}{l}\text { Brazil: Santa Catarina: } \\
\text { Garopaba }\end{array}$ & This study \\
\hline & & MZUSP 128425 & $\begin{array}{l}\text { Brazil: Paraná: Wenc- } \\
\text { eslau Brás }\end{array}$ & $\begin{array}{l}\text { This study; Thomé et } \\
\text { al., } 2010\end{array}$ \\
\hline R. achalensis & - & MACN 52406 & $\begin{array}{l}\text { Argentina: San Luis: } \\
\text { La Carolina }\end{array}$ & This study \\
\hline \multirow{2}{*}{ R. achavali } & \multirow{2}{*}{-} & MNHN-Uy 9301 & $\begin{array}{l}\text { Uruguay: Treinta y } \\
\text { Tres: Quebrada de los } \\
\text { Cuervos }\end{array}$ & This study \\
\hline & & ZVC 3801 & $\begin{array}{l}\text { Uruguay: Treinta y } \\
\text { Tres: Estancia Doña } \\
\text { Alba }\end{array}$ & Vallinoto et al., 2010 \\
\hline \multirow{2}{*}{ R. acrolopha } & \multirow{2}{*}{ - } & MAR 1425 & $\begin{array}{l}\text { Colombia: Chocó: } \\
\text { Unguia }\end{array}$ & This study \\
\hline & & MAR 1426 & $\begin{array}{l}\text { Colombia: Chocó: } \\
\text { Unguia }\end{array}$ & This study \\
\hline \multirow{4}{*}{ R. acutirostris } & \multirow{4}{*}{ "R. acutirostris" } & CORBIDI 4635 & Peru: Loreto: Andoas & This study \\
\hline & & MTR 36593 & $\begin{array}{l}\text { Brazil: Amazonas: Rio } \\
\text { Içá }\end{array}$ & This study \\
\hline & & MTR 36684 & $\begin{array}{l}\text { Brazil: Amazonas: Rio } \\
\text { Içá }\end{array}$ & This study \\
\hline & & QCAZ 10601 & $\begin{array}{l}\text { Ecuador: Orellana: } \\
\text { Parque Nacional Yas- } \\
\text { uní, Estación Cientí- } \\
\text { fica Yasuní PUCE }\end{array}$ & $\begin{array}{l}\text { Pramuk, 2006; Pramuk } \\
\text { et al., } 2008\end{array}$ \\
\hline
\end{tabular}


APPENDIX 1 continued

\begin{tabular}{|c|c|c|c|c|}
\hline \multicolumn{5}{|l|}{ RHINELLA } \\
\hline Current taxonomy & Updated taxonomy & Voucher & Locality & Sources \\
\hline R. acutirostris & "R. acutirostris" & QCAZ 28379 & $\begin{array}{l}\text { Ecuador: Sucumbios: } \\
\text { Reserva de Producción } \\
\text { Faunística Cuyabeno, } \\
\text { Playas de Cuyabeno }\end{array}$ & This study \\
\hline \multirow{5}{*}{ R. alata } & \multirow{5}{*}{ "R. alata" } & CH 9192 & $\begin{array}{l}\text { Panama: Colón: } \\
\text { Parque Nacional } \\
\text { Soberania }\end{array}$ & Santos et al., 2015 \\
\hline & & MAR 2574 & $\begin{array}{l}\text { Colombia: Tolima: } \\
\text { Rioblanco }\end{array}$ & This study \\
\hline & & MHUA 8415 & $\begin{array}{l}\text { Colombia: Antioquia: } \\
\text { Sonsón }\end{array}$ & This study \\
\hline & & QCAZ 11597 & $\begin{array}{l}\text { Ecuador: Esmeraldas: } \\
\text { Bosque Protector La } \\
\text { Chiquita }\end{array}$ & $\begin{array}{l}\text { Pramuk, 2006; Pramuk } \\
\text { et al., } 2008\end{array}$ \\
\hline & & QCAZ 13896 & $\begin{array}{l}\text { Ecuador: Cañar: } \\
\text { Manta Real }\end{array}$ & $\begin{array}{l}\text { Pramuk, 2006; Pramuk } \\
\text { et al., } 2008\end{array}$ \\
\hline R. amabilis & R. cf. amabilis & QCAZ 68471 & Peru: Cajamarca & This study \\
\hline R. amboroensis & R. quechua & MNK 5302 & $\begin{array}{l}\text { Bolivia: Santa Cruz: } \\
\text { Parque Nacional } \\
\text { Amboró }\end{array}$ & Frost et al., 2006 \\
\hline \multirow{3}{*}{ R. arborescandens } & \multirow{3}{*}{-} & CORBIDI 2020 & $\begin{array}{l}\text { Peru: Amazonas: } \\
\text { Bagua, Cataratas de } \\
\text { Camñopite }\end{array}$ & This study \\
\hline & & MUBI 14076 & $\begin{array}{l}\text { Peru: Amazonas: Bon- } \\
\text { gara }\end{array}$ & This study \\
\hline & & MUBI 14082 & $\begin{array}{l}\text { Peru: Amazonas: Bon- } \\
\text { gara }\end{array}$ & This study \\
\hline R. arenarum & - & AR 305 & Argentina & $\begin{array}{l}\text { Pramuk, 2006; Pramuk } \\
\text { et al., } 2008\end{array}$ \\
\hline \multirow{3}{*}{ R. arenarum arenarum } & \multirow{3}{*}{ R. arenarum } & MNHN-Uy 9935 & $\begin{array}{l}\text { Uruguay: Cerro Largo: } \\
\text { Laguna Merín }\end{array}$ & This study \\
\hline & & MACN 38639 & $\begin{array}{l}\text { Argentina: San Luis: } \\
\text { Lomas Blancas }\end{array}$ & $\begin{array}{l}\text { This study; Faivovich } \\
\text { et al., 2005; Frost et al., } \\
2006\end{array}$ \\
\hline & & MNCN-ADN 5972 & $\begin{array}{l}\text { Bolivia: Tarija: Reserva } \\
\text { Tariquia }\end{array}$ & This study \\
\hline $\begin{array}{l}R . \text { arenarum men- } \\
\text { docina }\end{array}$ & R. arenarum & MACN 49141 & $\begin{array}{l}\text { Argentina: Mendoza: } \\
\text { Tunuyán }\end{array}$ & This study \\
\hline \multirow{2}{*}{$R$. arequipensis } & \multirow{2}{*}{ R. spinulosa } & KU 214792 & $\begin{array}{l}\text { Peru: Arequipa: Zama- } \\
\text { cola, Cerro Colorado }\end{array}$ & $\begin{array}{l}\text { Pramuk, 2006; Pramuk } \\
\text { et al., } 2008\end{array}$ \\
\hline & & LGE 2516 & $\begin{array}{l}\text { Peru: Arequipa: Cañón } \\
\text { del Colca }\end{array}$ & This study \\
\hline R. arunco & - & KU 217369 & $\begin{array}{l}\text { Chile: Santiago: Run- } \\
\text { gue }\end{array}$ & $\begin{array}{l}\text { Pramuk, 2006; Pramuk } \\
\text { et al., } 2008\end{array}$ \\
\hline
\end{tabular}




\section{APPENDIX 1 continued}

\begin{tabular}{|c|c|c|c|c|}
\hline \multicolumn{5}{|l|}{ RHINELLA } \\
\hline Current taxonomy & Updated taxonomy & Voucher & Locality & Sources \\
\hline \multirow[b]{2}{*}{ R. atacamensis } & \multirow[b]{2}{*}{-} & AMNH 168401 & $\mathrm{n} / \mathrm{d}$ & Frost et al., 2006 \\
\hline & & KU 217352 & $\begin{array}{l}\text { Chile: Coquimbo: } \\
\text { Cuesta Pajonales }\end{array}$ & $\begin{array}{l}\text { Pramuk, 2006; Pramuk } \\
\text { et al., } 2008\end{array}$ \\
\hline \multirow{2}{*}{ R. azarai } & \multirow{2}{*}{-} & LGE 8710 & $\begin{array}{l}\text { Argentina: Misiones: } \\
\text { Candelaria }\end{array}$ & Pereyra et al., 2016a \\
\hline & & LGE 8711 & $\begin{array}{l}\text { Argentina: Misiones: } \\
\text { Candelaria }\end{array}$ & Pereyra et al., 2016a \\
\hline \multirow{5}{*}{ R. beebei } & \multirow{5}{*}{-} & CBA 5732 & $\begin{array}{l}\text { Venezuela: Bolivar: } \\
\text { Chivatón, Gran Sabana }\end{array}$ & Pereyra et al., 2016a \\
\hline & & ICN 55776 & $\begin{array}{l}\text { Colombia: Casanare: } \\
\text { Paz de Ariporo, Vereda } \\
\text { La Colombina, Finca } \\
\text { El Porvenir }\end{array}$ & Murphy et al., 2017 \\
\hline & & ICN 55784 & $\begin{array}{l}\text { Colombia: Casanare: } \\
\text { Trinidad, Vereda La } \\
\text { Cañada, Finca La Pal- } \\
\text { mita }\end{array}$ & Murphy et al., 2017 \\
\hline & & $\mathrm{nv}$ & $\begin{array}{l}\text { Venezuela: Amazonas: } \\
\text { Puerto Ayacucho }\end{array}$ & Pereyra et al., 2016a \\
\hline & & UWIZM 2012.27.72.3 & $\begin{array}{l}\text { Trinidad and Tobago: } \\
\text { Trinidad: Trincity } \\
\text { Central Road }\end{array}$ & Murphy et al., 2017 \\
\hline \multirow{2}{*}{ R. bergi } & \multirow{2}{*}{-} & LGE 8723 & $\begin{array}{l}\text { Argentina: Formosa: } \\
\text { Pilcomayo, Palma Sola }\end{array}$ & Pereyra et al., 2016a \\
\hline & & MACN 46555 & $\begin{array}{l}\text { Argentina: Chaco: San } \\
\text { Fernando }\end{array}$ & Pereyra et al., 2016a \\
\hline \multirow[t]{2}{*}{ R. bernardoi } & \multirow[t]{2}{*}{-} & FML 23921 & $\begin{array}{l}\text { Argentina: San Juan: } \\
\text { Parque Provincial } \\
\text { Ischigualasto }\end{array}$ & Pereyra et al., 2016a \\
\hline & & UNSJ 5046 & $\begin{array}{l}\text { Argentina: San Juan: } \\
\text { Caucete }\end{array}$ & Pereyra et al., 2016a \\
\hline \multirow{2}{*}{ R. casconi } & \multirow{2}{*}{-} & CFBH 22863 & $\begin{array}{l}\text { Brazil: Ceará: } \\
\text { Guaramiranga }\end{array}$ & This study \\
\hline & & CFBH 22865 & $\begin{array}{l}\text { Brazil: Ceará: } \\
\text { Guaramiranga }\end{array}$ & This study \\
\hline R. castaneotica & - & LSUMZ 17429 & $\begin{array}{l}\text { Brazil: Pará: } 100 \text { km S } \\
\text { Santarém }\end{array}$ & $\begin{array}{l}\text { Pramuk, 2006; Pramuk } \\
\text { et al., } 2008\end{array}$ \\
\hline \multirow{3}{*}{ R. cf. castaneotica } & \multirow{3}{*}{ "R. castaneotica" } & NMP6V 74261 & $\begin{array}{l}\text { Bolivia: Pando, Fed- } \\
\text { erico Román: Santa } \\
\text { Crucito }\end{array}$ & Moravec et al., 2014 \\
\hline & & BM 131 & $\begin{array}{l}\text { French Guiana: } \\
\text { Mataroni }\end{array}$ & Fouquet et al., 2007c \\
\hline & & ZUFG 8171 & $\begin{array}{l}\text { Brazil: Acre: Boca do } \\
\text { Acre }\end{array}$ & This study \\
\hline
\end{tabular}


APPENDIX 1 continued

\begin{tabular}{|c|c|c|c|c|}
\hline \multicolumn{5}{|l|}{ RHINELLA } \\
\hline Current taxonomy & Updated taxonomy & Voucher & Locality & Sources \\
\hline R. cf. castaneotica & R. proboscidea & MTR 10003 & $\begin{array}{l}\text { Brazil: Amazonas: } \\
\text { Lago Cipotuba }\end{array}$ & Fouquet et al., 2012a \\
\hline \multirow{2}{*}{ R. centralis } & \multirow{2}{*}{-} & CH 9383 & $\begin{array}{l}\text { Panama: Coclé: Valle } \\
\text { de Antón }\end{array}$ & Pereyra et al., 2016a \\
\hline & & MVUP 2305 & $\begin{array}{l}\text { Panama: Coclé: Valle } \\
\text { de Antón }\end{array}$ & Pereyra et al., 2016a \\
\hline \multirow[b]{2}{*}{ R. ceratophrys } & \multirow[b]{2}{*}{ Rhaebo ceratophrys } & JMP 2284 & $\begin{array}{l}\text { Colombia: Amazonas: } \\
\text { Leticia }\end{array}$ & This study \\
\hline & & QCAZ 40240 & $\begin{array}{l}\text { Ecuador: Sucumbios: } \\
\text { Sansa Huari, Comuna } \\
\text { Singue } 1\end{array}$ & This study \\
\hline \multirow{3}{*}{ R. cerradensis } & \multirow{3}{*}{-} & CFBH 20517 & $\begin{array}{l}\text { Brazil: Bahia: Jabo- } \\
\text { randi }\end{array}$ & This study \\
\hline & & CHUNB 38671 & $\begin{array}{l}\text { Brazil: Distrito Fed- } \\
\text { eral: Brasília }\end{array}$ & This study \\
\hline & & CHUNB 39953 & $\begin{array}{l}\text { Brazil: Distrito Fed- } \\
\text { eral: Brasília }\end{array}$ & This study \\
\hline \multirow{3}{*}{ R. aff. cerradensis } & \multirow{3}{*}{-} & LGE 19096 & $\begin{array}{l}\text { Argentina: Misiones: } \\
\text { Posadas }\end{array}$ & This study \\
\hline & & LGE 19103 & $\begin{array}{l}\text { Argentina: Misiones: } \\
\text { Posadas }\end{array}$ & This study \\
\hline & & MNHN-Uy 9514 & $\begin{array}{l}\text { Uruguay: Rivera: } \\
\text { Pueblo Madera }\end{array}$ & This study \\
\hline R. chavin & - & MTD 43789 & $\begin{array}{l}\text { Peru: Huánuco: } \\
\text { Pachitea, Palma Pampa }\end{array}$ & $\begin{array}{l}\text { Pramuk, 2006; Pramuk } \\
\text { et al., } 2008\end{array}$ \\
\hline \multirow{2}{*}{ R. crucifer } & \multirow{2}{*}{-} & CFBH 2867 & $\begin{array}{l}\text { Brazil: Espírito Santo: } \\
\text { Aracruz }\end{array}$ & $\begin{array}{l}\text { This study; Thomé et } \\
\text { al., } 2010\end{array}$ \\
\hline & & CFBH 24630 & $\begin{array}{l}\text { Brazil: Bahia: Cama- } \\
\text { can }\end{array}$ & $\begin{array}{l}\text { This study; Thomé et } \\
\text { al., } 2010\end{array}$ \\
\hline \multirow{3}{*}{ R. dapsilis } & \multirow{3}{*}{-} & QCAZ 17719 & Ecuador: Napo: Cando & $\begin{array}{l}\text { Pramuk, 2006; Pramuk } \\
\text { et al., } 2008\end{array}$ \\
\hline & & QCAZ 38892 & $\begin{array}{l}\text { Ecuador: Pastaza: Vil- } \\
\text { lano }\end{array}$ & Santos et al., 2015 \\
\hline & & QCAZ 43967 & $\begin{array}{l}\text { Ecuador: Orellana: } \\
\text { Parque Nacional Yas- } \\
\text { uní, Comunidad } \\
\text { Añangu, Río Napo }\end{array}$ & This study \\
\hline \multirow{4}{*}{ R. cf. dapsilis } & \multirow{4}{*}{ R. dapsilis } & CORBIDI 1969 & $\begin{array}{l}\text { Peru: Amazonas: } \\
\text { Bagua, Chonza Alta }\end{array}$ & This study \\
\hline & & MTR 6313 & $\begin{array}{l}\text { Brazil: Pará: Serra do } \\
\text { Kukoinhokren }\end{array}$ & Fouquet et al., 2012a \\
\hline & & MZUSP 139598 & Brazil: Pará & This study \\
\hline & & QCAZ 38621 & $\begin{array}{l}\text { Ecuador: Pastaza, Vil- } \\
\text { lano }\end{array}$ & Santos et al., 2015 \\
\hline
\end{tabular}


APPENDIX 1 continued

\begin{tabular}{|c|c|c|c|c|}
\hline \multicolumn{5}{|l|}{ RHINELLA } \\
\hline Current taxonomy & Updated taxonomy & Voucher & Locality & Sources \\
\hline R. cf. dapsilis & R. dapsilis & QCAZ 39474 & $\begin{array}{l}\text { Ecuador: Orellana: } \\
\text { Alta Florencia, } 6.5 \mathrm{~km} \\
\text { NO de Nuevo Roca- } \\
\text { fuerte, Río Napo }\end{array}$ & This study \\
\hline \multirow{3}{*}{ R. diptycha } & \multirow{3}{*}{ - } & KU 289057 & $\begin{array}{l}\text { Paraguay: Concepción: } \\
\text { Parque Nacional Ser- } \\
\text { ranía San Luis }\end{array}$ & Mulcahy et al., 2006 \\
\hline & & MACN 51118 & $\begin{array}{l}\text { Argentina: Santiago } \\
\text { del Estero: Guasayán }\end{array}$ & $\begin{array}{l}\text { This study; Frost et al., } \\
2006\end{array}$ \\
\hline & & MNCN-ADN 6044 & $\begin{array}{l}\text { Bolivia: La Paz: San } \\
\text { José de Uchupiamonas }\end{array}$ & $\begin{array}{l}\text { This study; Vallinoto } \\
\text { et al., } 2010\end{array}$ \\
\hline R. cf. diptycha & R. diptycha & LGE 9867 & $\begin{array}{l}\text { Argentina: Misiones: } \\
\text { Capital, Fachinal }\end{array}$ & This study \\
\hline \multirow[b]{2}{*}{$R$. dorbignyi } & \multirow[b]{2}{*}{ - } & MACN 43695 & $\begin{array}{l}\text { Argentina: Buenos } \\
\text { Aires: Dolores }\end{array}$ & Pereyra et al., 2016a \\
\hline & & MNHN-Uy 9492 & $\begin{array}{l}\text { Uruguay: Treinta y } \\
\text { Tres: Bañado de los } \\
\text { Oliveras }\end{array}$ & Pereyra et al., 2016a \\
\hline R. cf. dorbignyi & R. dorbignyi & CFBH 14062 & $\begin{array}{l}\text { Brazil: Rio Grande do } \\
\text { Sul: Rio Grande }\end{array}$ & Pereyra et al., $2016 a$ \\
\hline \multirow{2}{*}{ R. fernandezae } & \multirow{2}{*}{ R. dorbignyi } & LGE 8717 & $\begin{array}{l}\text { Argentina: Corrientes: } \\
\text { General Paz, Itá Ibaté }\end{array}$ & Pereyra et al., 2016a \\
\hline & & LGE 8718 & $\begin{array}{l}\text { Argentina: Santa Fe: } 9 \\
\text { de Julio, Tostado }\end{array}$ & Pereyra et al., $2016 a$ \\
\hline \multirow{5}{*}{ R. festae } & \multirow{5}{*}{-} & CORBIDI 7505 & $\begin{array}{l}\text { Peru: Loreto: Datem } \\
\text { del Marañon, Morona }\end{array}$ & This study \\
\hline & & KU 217501 & $\begin{array}{l}\text { Ecuador: Pastaza: } \\
\text { Montalvo }\end{array}$ & $\begin{array}{l}\text { Pramuk, 2006; Pramuk } \\
\text { et al., 2008; Mendelson } \\
\text { et al., } 2011\end{array}$ \\
\hline & & QCAZ 18203 & $\begin{array}{l}\text { Ecuador: Napo: } \\
\text { Estación Biológica } \\
\text { Jatun Sacha }\end{array}$ & Santos et al., 2015 \\
\hline & & QCAZ 41490 & $\begin{array}{l}\text { Ecuador: Zamora: } \\
\text { Miazi Alto }\end{array}$ & This study \\
\hline & & QCAZ 46457 & $\begin{array}{l}\text { Ecuador: Morona: } \\
\text { Santiago, Nuevo Israel }\end{array}$ & This study \\
\hline R. fissippes & - & MNCN-ADN 6310 & $\begin{array}{l}\text { Bolivia: Beni-Cocha- } \\
\text { bamba: Santo } \\
\text { Domingo, Parque } \\
\text { Nacional Isiboro- } \\
\text { Sécure }\end{array}$ & This study \\
\hline \multirow{2}{*}{ R. gallardo $i$} & \multirow{2}{*}{ - } & LGE 4546 & $\begin{array}{l}\text { Argentina: Jujuy: Man - } \\
\text { uel Belgrano }\end{array}$ & This study \\
\hline & & LGE 4735 & $\begin{array}{l}\text { Argentina: Jujuy: Abra } \\
\text { Colorada }\end{array}$ & This study \\
\hline
\end{tabular}


APPENDIX 1 continued

\begin{tabular}{|c|c|c|c|c|}
\hline \multicolumn{5}{|l|}{ RHINELLA } \\
\hline Current taxonomy & Updated taxonomy & Voucher & Locality & Sources \\
\hline \multirow{3}{*}{ R. gildae } & \multirow{3}{*}{ R. dapsilis } & CFBH 11400 & $\begin{array}{l}\text { Brazil: Tocantins: } \\
\text { Babaçulândia }\end{array}$ & This study \\
\hline & & ESTR 173 & $\begin{array}{l}\text { Brazil: Maranhão: } \\
\text { Carolina }\end{array}$ & Fouquet et al., $2012 b$ \\
\hline & & URCA 12651 & $\begin{array}{l}\text { Brazil: Maranhão: São } \\
\text { Pedro da Água Branca }\end{array}$ & Avila et al., 2018 \\
\hline \multirow{2}{*}{ R. granulosa } & \multirow{2}{*}{-} & CFBH 7341 & $\begin{array}{l}\text { Brazil: Alagoas: Passo } \\
\text { de Camaragibe }\end{array}$ & Pereyra et al., 2016a \\
\hline & & CFBH 18706 & $\begin{array}{l}\text { Brazil: Espírito Santo: } \\
\text { Linhares }\end{array}$ & Pereyra et al., 2016a \\
\hline \multirow{3}{*}{ R. henseli } & \multirow{3}{*}{-} & CFBH 20117 & $\begin{array}{l}\text { Brazil: Rio Grande do } \\
\text { Sul: Catiporã }\end{array}$ & This study \\
\hline & & MNRJ 33006 & $\begin{array}{l}\text { Brazil: Rio Grande do } \\
\text { Sul: Mato Castelhano }\end{array}$ & $\begin{array}{l}\text { Thomé et al., 2010; } \\
\text { Pereyra et al., 2016a }\end{array}$ \\
\hline & & UFRGS 3569 & $\begin{array}{l}\text { Brazil: Rio Grande do } \\
\text { Sul: Nova Roma do Sul }\end{array}$ & This study \\
\hline \multirow{4}{*}{ R. hoogmoedi } & \multirow{4}{*}{-} & CFBH 13286 & Brazil: Bahia: Una & This study \\
\hline & & CFBH 15962 & $\begin{array}{l}\text { Brazil: São Paulo: San- } \\
\text { tos }\end{array}$ & This study \\
\hline & & MTR 16199 & $\begin{array}{l}\text { Brazil: Bahia: Serra } \\
\text { Bonita, Camacan }\end{array}$ & Fouquet et al., 2012b \\
\hline & & ZUECDCC 3393 & $\begin{array}{l}\text { Brazil: Rio de Janeiro: } \\
\text { Magé, Santo Aleixo }\end{array}$ & Pauly et al., 2004 \\
\hline \multirow[t]{8}{*}{ R. horribilis } & \multirow[t]{4}{*}{-} & KRL 744 & $\begin{array}{l}\text { Panamá: Coclé: El } \\
\text { Cope }\end{array}$ & Crawford et al., 2010 \\
\hline & & KU 289750 & $\begin{array}{l}\text { El Salvador: Ahuacha- } \\
\text { pan: Parque Nacional } \\
\text { El Imposible }\end{array}$ & $\begin{array}{l}\text { Mulcahy et al., 2006; } \\
\text { Pramuk, 2006; Pramuk } \\
\text { et al., } 2008\end{array}$ \\
\hline & & MAR 2057 & $\begin{array}{l}\text { Colombia: Valle del } \\
\text { Cauca: Dagua }\end{array}$ & This study \\
\hline & & UTA 54882 & Mexico: Veracruz & Mulcahy et al., 2006 \\
\hline & \multirow[t]{4}{*}{ Rhinella sp. 1} & KU 202274 & $\begin{array}{l}\text { Ecuador: Pichincha: } \\
\text { Tinalandia }\end{array}$ & Pauly et al., 2004 \\
\hline & & KU 217482 & $\begin{array}{l}\text { Ecuador: Loja: Vilca- } \\
\text { bamba }\end{array}$ & $\begin{array}{l}\text { Mulcahy et al., 2006; } \\
\text { Pramuk, 2006; Pramuk } \\
\text { et al., } 2008\end{array}$ \\
\hline & & QCAZ 47444 & $\begin{array}{l}\text { Ecuador: Loja: San } \\
\text { Bernabé }\end{array}$ & This study \\
\hline & & QCAZ 50698 & $\begin{array}{l}\text { Ecuador: Manabí: } \\
\text { Puerto Cayo }\end{array}$ & This study \\
\hline R. humboldti & "R. humboldti" & AJC 3533 & $\begin{array}{l}\text { Colombia: Santander: } \\
\text { San Vicente de Chu- } \\
\text { curi, Reserva El Arbo- } \\
\text { retum }\end{array}$ & Guarnizo et al., 2015 \\
\hline
\end{tabular}


APPENDIX 1 continued

\begin{tabular}{|c|c|c|c|c|}
\hline \multicolumn{5}{|l|}{ RHINELLA } \\
\hline Current taxonomy & Updated taxonomy & Voucher & Locality & Sources \\
\hline R. humboldti & "R. humboldti" & CZUT 1717 & $\begin{array}{l}\text { Colombia: Tolima: } \\
\text { Prado, Vereda El Cai- } \\
\text { mán, Represa hidro- } \\
\text { eléctrica Hidroprado }\end{array}$ & Murphy et al., 2017 \\
\hline \multirow{4}{*}{ R. icterica } & \multirow{4}{*}{ "R. icterica" } & CFBH 11027 & $\begin{array}{l}\text { Brazil: Santa Catarina: } \\
\text { Bom Jardim da Serra, } \\
\text { Serra do Rio do Rastro }\end{array}$ & $\begin{array}{l}\text { This study; Thomé et } \\
\text { al., } 2010\end{array}$ \\
\hline & & CFBH 13965 & $\begin{array}{l}\text { Brazil: Rio de Janeiro: } \\
\text { Petrópolis }\end{array}$ & This study \\
\hline & & CFBH 27410 & $\begin{array}{l}\text { Brazil: Rio de Janeiro: } \\
\text { Parque Estadual dos } \\
\text { Três Picos }\end{array}$ & This study \\
\hline & & CFBH 38392 & $\begin{array}{l}\text { Brazil: Minas Gerais: } \\
\text { Rio Preto }\end{array}$ & This study \\
\hline R. cf. icterica & "R. icterica" & MACN 43789 & $\begin{array}{l}\text { Argentina: Misiones: } \\
\text { San Vicente }\end{array}$ & This study \\
\hline \multirow{4}{*}{ R. inca } & \multirow{4}{*}{ - } & CORBIDI 6920 & $\begin{array}{l}\text { Peru: Ayacucho: San } \\
\text { Antonio, La Mar }\end{array}$ & This study \\
\hline & & LGE 2554 & $\begin{array}{l}\text { Peru: Cusco: } \\
\text { Urubamba }\end{array}$ & This study \\
\hline & & MNCN 44405 & $\begin{array}{l}\text { Peru: Cusco: La Con- } \\
\text { vención, Río Kimbiri, } \\
\text { Comunidad } \\
\text { Machiguenga } \\
\text { Pomoreni }\end{array}$ & This study \\
\hline & & MNCN 44406 & $\begin{array}{l}\text { Peru: Cusco: La Con- } \\
\text { vención: Río Kimbiri, } \\
\text { Comunidad } \\
\text { Machiguenga } \\
\text { Pomoreni }\end{array}$ & This study \\
\hline \multirow{3}{*}{ R. inopina } & \multirow{3}{*}{-} & CHUNB 51110 & $\begin{array}{l}\text { Brazil: Bahia: São } \\
\text { Desidério }\end{array}$ & This study \\
\hline & & MZUSP 142356 & $\begin{array}{l}\text { Brazil: Minas Gerais: } \\
\text { Januaría }\end{array}$ & This study \\
\hline & & MZUSP 142094 & $\begin{array}{l}\text { Brazil: Minas Gerais: } \\
\text { Januaría }\end{array}$ & This study \\
\hline \multirow{2}{*}{ R. jimi } & \multirow{2}{*}{ R. diptycha } & CFBH 19335 & Brazil: Bahia: Maracás & This study \\
\hline & & CFBH 19523 & Brazil: Bahia: Maracás & This study \\
\hline R. justinianoi & - & MNCN-ADN 6065 & $\begin{array}{l}\text { Bolivia: Santa Cruz: } \\
\text { Florida, La Yunga de } \\
\text { Mairana }\end{array}$ & This study \\
\hline \multirow{3}{*}{ R. leptoscelis } & \multirow{2}{*}{ - } & MUBI 5976 & Peru: Puno: Carabaya & This study \\
\hline & & MUBI 5989 & Peru: Puno: Carabaya & This study \\
\hline & Rhinella sp. 2 & CORBIDI 7266 & $\begin{array}{l}\text { Peru: Oxapampa: Huan- } \\
\text { cabamba, Huampal }\end{array}$ & This study \\
\hline
\end{tabular}




\section{APPENDIX 1 continued}

\begin{tabular}{|c|c|c|c|c|}
\hline RHINELLA & & & & \\
\hline Current taxonomy & Updated taxonomy & Voucher & Locality & Sources \\
\hline \multirow{3}{*}{ R. leptoscelis } & \multirow{3}{*}{ Rhinella sp. 2} & MUBI 14523 & $\begin{array}{l}\text { Peru: Pasco: Oxa- } \\
\text { pampa }\end{array}$ & This study \\
\hline & & MUSM 31150 & $\begin{array}{l}\text { Peru: Pasco: Oxa- } \\
\text { pampa, Oxapampa }\end{array}$ & Moravec et al., 2014 \\
\hline & & NMP6V 74749 & $\begin{array}{l}\text { Peru: Pasco: Oxa- } \\
\text { pampa, Quebrada San } \\
\text { Alberto }\end{array}$ & Moravec et al., 2014 \\
\hline \multirow{3}{*}{ R. lescurei } & \multirow{3}{*}{-} & AF 1613 & $\begin{array}{l}\text { French Guiana: St } \\
\text { Laurent Du Maroni } \\
\text { Saul }\end{array}$ & This study \\
\hline & & MC 5 & $\begin{array}{l}\text { French Guiana: Cis- } \\
\text { ame }\end{array}$ & Fouquet et al., 2007c \\
\hline & & MNHN-Fr 2006.2611 & $\begin{array}{l}\text { French Guiana: Haute } \\
\text { Wanapi }\end{array}$ & Fouquet et al., 2012a \\
\hline \multirow{4}{*}{ R. lilyrodriguezae } & \multirow{4}{*}{-} & CORBIDI 6778 & $\begin{array}{l}\text { Peru: San Martín: } \\
\text { Mariscal Caceres }\end{array}$ & This study \\
\hline & & CORBIDI 6780 & $\begin{array}{l}\text { Peru: San Martín: } \\
\text { Mariscal Caceres }\end{array}$ & This study \\
\hline & & CORBIDI 8839 & $\begin{array}{l}\text { Peru: San Martín: Alto } \\
\text { Biavo, Parque Nacional } \\
\text { Cordillera Azul }\end{array}$ & This study \\
\hline & & MUSM 32205 & $\begin{array}{l}\text { Peru: San Martín: Alto } \\
\text { Biavo, Parque Nacional } \\
\text { Cordillera Azul }\end{array}$ & Cusi et al., 2017 \\
\hline \multirow{3}{*}{ R. limensis } & \multirow{3}{*}{-} & $\mathrm{nv}$ & Peru: Lima: Lima & This study \\
\hline & & KU 215587 & $\begin{array}{l}\text { Peru: Ancash: Casma, } \\
\text { Rio Casma, Casma }\end{array}$ & $\begin{array}{l}\text { Pramuk, 2006; Pramuk } \\
\text { et al., } 2008\end{array}$ \\
\hline & & RGP 4719 & Peru: Arequipa: Islay & This study \\
\hline \multirow{3}{*}{ R. lindae } & \multirow{3}{*}{-} & MAR 3330 & $\begin{array}{l}\text { Colombia Antioquia: } \\
\text { Parque Nacional Natu- } \\
\text { ral Las Orquídeas }\end{array}$ & This study \\
\hline & & MAR 3431 & $\begin{array}{l}\text { Colombia: Antioquia: } \\
\text { Parque Nacional Natu- } \\
\text { ral Las Orquídeas }\end{array}$ & This study \\
\hline & & MAR 3432 & $\begin{array}{l}\text { Colombia: Antioquia: } \\
\text { Parque Nacional Natu- } \\
\text { ral Las Orquídeas }\end{array}$ & This study \\
\hline \multirow{2}{*}{ R. macrorhina } & \multirow{2}{*}{-} & MAR 2867 & $\begin{array}{l}\text { Colombia: Caldas: } \\
\text { Parque Nacional Selva } \\
\text { de Florencia }\end{array}$ & This study \\
\hline & & MAR 2903 & $\begin{array}{l}\text { Colombia: Caldas: } \\
\text { Parque Nacional Selva } \\
\text { de Florencia }\end{array}$ & This study \\
\hline
\end{tabular}


APPENDIX 1 continued

\begin{tabular}{|c|c|c|c|c|}
\hline \multicolumn{5}{|l|}{ RHINELLA } \\
\hline Current taxonomy & Updated taxonomy & Voucher & Locality & Sources \\
\hline \multirow{2}{*}{ R. macrorhina } & \multirow{2}{*}{ - } & MHUA 8319 & $\begin{array}{l}\text { Colombia: Antioquia: } \\
\text { Vereda Santa Rita, } \\
\text { Guatapé }\end{array}$ & This study \\
\hline & & MHUA 10262 & $\begin{array}{l}\text { Colombia: Antioquia: } \\
\text { Vereda La Esperanza, } \\
\text { El Carmen de Viboral }\end{array}$ & This study \\
\hline R. magnussoni & - & APL 20530 & Brazil: Pará: Treviso & This study \\
\hline \multirow{2}{*}{ R. major } & \multirow{2}{*}{ - } & LGE 8720 & $\begin{array}{l}\text { Argentina: Salta: } \\
\text { Rivadavia, El Ocultar }\end{array}$ & Pereyra et al., $2016 a$ \\
\hline & & MNCN-ADN 6232 & $\begin{array}{l}\text { Bolivia: Cochabamba: } \\
\text { Chapare }\end{array}$ & Pereyra et al., 2016a \\
\hline \multirow{4}{*}{ R. manu } & \multirow{2}{*}{-} & MUBI 11372 & $\begin{array}{l}\text { Peru: Cusco: Trocha } \\
\text { Unión }\end{array}$ & This study \\
\hline & & MNCN-ADN 20672 & $\begin{array}{l}\text { Peru: Cusco: Parque } \\
\text { Nacional Manu }\end{array}$ & This study \\
\hline & \multirow{2}{*}{ Rhinella sp. 3} & CORBIDI 5152 & $\begin{array}{l}\text { Peru: Madre de Dios: } \\
\text { Tambopata, Baltimore }\end{array}$ & This study \\
\hline & & MUBI 10487 & $\begin{array}{l}\text { Peru: Cusco: La Con- } \\
\text { vencion }\end{array}$ & This study \\
\hline \multirow{13}{*}{ R. cf. margaritifera } & \multirow[t]{2}{*}{ R. dapsilis } & IWU 334 & $\begin{array}{l}\text { Peru: Junín: Chan- } \\
\text { chamayo, Ayte, Bosque } \\
\text { de Protección Pui Pui }\end{array}$ & $\begin{array}{l}\text { This study; Cusi et al., } \\
2017\end{array}$ \\
\hline & & MUSM 32715 & $\begin{array}{l}\text { Peru: Cusco: Oxa- } \\
\text { pampa }\end{array}$ & $\begin{array}{l}\text { This study; Cusi et al., } \\
2017\end{array}$ \\
\hline & Rhinella sp. 6 & ANDES 1723 & $\begin{array}{l}\text { Colombia: Amazonas: } \\
\text { Leticia }\end{array}$ & This study \\
\hline & Rhinella sp. 7 & PD 16 & $\begin{array}{l}\text { Brazil: Amazonas: Rio } \\
\text { Içá }\end{array}$ & This study \\
\hline & Rhinella sp. 10 & QCAZ 42269 & $\begin{array}{l}\text { Ecuador: Napo: } \\
\text { Reserva Ecológica } \\
\text { Yachana }\end{array}$ & This study \\
\hline & Rhinella sp. 11 & CHUNB 32342 & $\begin{array}{l}\text { Brazil: Amazonas: } \\
\text { Humaitá }\end{array}$ & This study \\
\hline & \multirow{3}{*}{ Rhinella sp. 12} & NMP6V 74260 & $\begin{array}{l}\text { Bolivia: Pando: } \\
\text { Manuripi, San Antonio }\end{array}$ & Moravec et al., 2014 \\
\hline & & ROM 40103 & $\begin{array}{l}\text { Peru: Madre de Dios: } \\
\text { Tambopata }\end{array}$ & Fouquet et al., 2012b \\
\hline & & USNM 268828 & $\begin{array}{l}\text { Peru: Madre de Dios: } \\
\text { Reserva Tambopata }\end{array}$ & $\begin{array}{l}\text { Pramuk, 2006; Pramuk } \\
\text { et al., } 2008\end{array}$ \\
\hline & \multirow{2}{*}{ Rhinella sp. 13} & CORBIDI 5840 & Peru: Loreto: Curupa & Santos et al., 2015 \\
\hline & & CORBIDI 5848 & Peru: Loreto: Curupa & This study \\
\hline & \multirow{2}{*}{ Rhinella sp. 14} & MUBI 6374 & Peru: Loreto: Mayna & This study \\
\hline & & MUBI 14775 & Peru: Ucayali & This study \\
\hline
\end{tabular}




\section{APPENDIX 1 continued}

\begin{tabular}{|c|c|c|c|c|}
\hline RHINELLA & & & & \\
\hline Current taxonomy & Updated taxonomy & Voucher & Locality & Sources \\
\hline \multirow{6}{*}{ R. cf. margaritifera } & \multirow{6}{*}{ Rhinella sp. 14} & MUBI 14776 & Peru: Ucayali & This study \\
\hline & & CORBIDI 5468 & $\begin{array}{l}\text { Peru: Cusco: La Con- } \\
\text { vención }\end{array}$ & This study \\
\hline & & KU 215145 & $\begin{array}{l}\text { Peru: Madre de Dios: } \\
\text { Cusco Amazónico, } \\
\text { Puerto Maldonado }\end{array}$ & $\begin{array}{l}\text { Pramuk, 2006; Pramuk } \\
\text { et al., } 2008\end{array}$ \\
\hline & & KU 215146 & $\begin{array}{l}\text { Peru: Madre de Dios: } \\
\text { Cusco Amazónico, } \\
\text { Puerto Maldonado }\end{array}$ & Mendelson et al., 2011 \\
\hline & & MNCN-ADN 20639 & $\begin{array}{l}\text { Peru: Puno: Carabaya, } \\
\text { between Puerto Leguia } \\
\text { and San Gabán }\end{array}$ & This study \\
\hline & & NMP6V 74915 & $\begin{array}{l}\text { Peru: Ucayali: } \\
\text { Pucallpa, Masisea }\end{array}$ & Moravec et al., 2014 \\
\hline \multirow{3}{*}{ R. marina } & \multirow{3}{*}{-} & MAR 1982 & $\begin{array}{l}\text { Colombia: Caquetá: } \\
\text { Florencia }\end{array}$ & This study \\
\hline & & SBH 190696 & $\begin{array}{l}\text { Jamaica: St. Mary: } \\
\text { Galina }\end{array}$ & Pramuk et al., 2001 \\
\hline & & VUB 1965 & Suriname & $\begin{array}{l}\text { van Bocxlaer et al., 2009; } \\
\text { Liedtke et al., } 2016\end{array}$ \\
\hline \multirow{3}{*}{ R. martyi } & \multirow{3}{*}{ R. margaritifera } & MC 156 & $\begin{array}{l}\text { French Guiana: Tri- } \\
\text { jonction }\end{array}$ & Fouquet et al., 2007c \\
\hline & & MNHN-Fr 2006.2602 & $\begin{array}{l}\text { Suriname: Brownsberg } \\
\text { Nature Park }\end{array}$ & Fouquet et al., 2012a \\
\hline & & MW 1006 & Guyana & $\begin{array}{l}\text { van Bocxlaer et al., } \\
\text { 2009; van Bocxlaer et } \\
\text { al., } 2010\end{array}$ \\
\hline \multirow{2}{*}{ R. merianae } & \multirow{2}{*}{-} & CFBH 16641 & $\begin{array}{l}\text { Brazil: Amazonas: } \\
\text { Manaus, Reserva } \\
\text { Ducke }\end{array}$ & Pereyra et al., 2016a \\
\hline & & MTR 20517 & $\begin{array}{l}\text { Brazil: Roraima: Esta- } \\
\text { ção Ecológica de } \\
\text { Maracá }\end{array}$ & This study \\
\hline \multirow[b]{2}{*}{ R. mirandaribeiroi } & \multirow[b]{2}{*}{-} & CFBH 10254 & $\begin{array}{l}\text { Brazil: Tocantins: Ara- } \\
\text { guacema }\end{array}$ & Pereyra et al., 2016a \\
\hline & & СFBH 13849 & $\begin{array}{l}\text { Brazil: Marãnhao: } \\
\text { Parque Nacional dos } \\
\text { Lençóis Maranhenses }\end{array}$ & Pereyra et al., 2016a \\
\hline R. multiverrucosa & R. cf. multiverrucosa & MUBI 11455 & Peru: Huanúnco & This study \\
\hline \multirow{2}{*}{ R. nesiotes } & \multirow{2}{*}{-} & CORBIDI 8122 & $\begin{array}{l}\text { Peru: Cusco: La Con- } \\
\text { vención, Echarati }\end{array}$ & This study \\
\hline & & CORBIDI 13953 & $\begin{array}{l}\text { Peru: Huanúco: Puerto } \\
\text { Inca, Yuyapichis }\end{array}$ & This study \\
\hline R. nicefori & R. cf. nicefori & MHUA 4793 & $\begin{array}{l}\text { Colombia: Antioquia: } \\
\text { Belmira }\end{array}$ & This study \\
\hline
\end{tabular}


APPENDIX 1 continued

\begin{tabular}{|c|c|c|c|c|}
\hline RHINELLA & & & & \\
\hline Current taxonomy & Updated taxonomy & Voucher & Locality & Sources \\
\hline \multirow{4}{*}{ R. ocellata } & \multirow{4}{*}{-} & CFBH 26592 & $\begin{array}{l}\text { Brazil: Maranhão: } \\
\text { Barreirinhas }\end{array}$ & This study \\
\hline & & LAJ 210 & $\begin{array}{l}\text { Brazil: Tocantins: } \\
\text { Parque Estadual do } \\
\text { Lajeado }\end{array}$ & Fouquet et al., 2012b \\
\hline & & MZUSP 103261 & Brazil: Tocantins: Peixe & $\begin{array}{l}\text { Pramuk, 2006; Pramuk } \\
\text { et al., } 2008\end{array}$ \\
\hline & & ZUFG 8519 & $\begin{array}{l}\text { Brazil: Goiás: Morrin- } \\
\text { hos }\end{array}$ & This study \\
\hline \multirow{8}{*}{ R. ornata } & \multirow{8}{*}{-} & CFBH 18815 & $\begin{array}{l}\text { Brazil: Rio de Janeiro: } \\
\text { Parque Nacional da } \\
\text { Serra dos Órgãos }\end{array}$ & $\begin{array}{l}\text { This study; Thomé et } \\
\text { al., } 2010\end{array}$ \\
\hline & & CFBH 38375 & $\begin{array}{l}\text { Brazil: Rio de Janeiro: } \\
\text { Visconde de Mauá }\end{array}$ & This study \\
\hline & & LGE 6503 & $\begin{array}{l}\text { Argentina: Misiones: } \\
\text { Cuña Pirú }\end{array}$ & This study \\
\hline & & LGE 8729 & $\begin{array}{l}\text { Argentina: Misiones: } \\
\text { Profundidad }\end{array}$ & This study \\
\hline & & LGE 19020 & $\begin{array}{l}\text { Argentina: Misiones: } \\
\text { El Soberbio }\end{array}$ & This study \\
\hline & & LGE 19027 & $\begin{array}{l}\text { Argentina: Misiones: } \\
\text { Capital }\end{array}$ & This study \\
\hline & & USNM 303015 & $\begin{array}{l}\text { Brazil: São Paulo: } \\
\text { Salesópolis, Serra do } \\
\text { Mar }\end{array}$ & $\begin{array}{l}\text { Mulcahy et al., 2006; } \\
\text { Pramuk, } 2006\end{array}$ \\
\hline & & ZUECDCC 3392 & $\begin{array}{l}\text { Brazil: Rio de Janeiro: } \\
\text { Magé, Campo de } \\
\text { Escoteiros, Santo } \\
\text { Aleixo }\end{array}$ & $\begin{array}{l}\text { Pauly et al., 2004; } \\
\text { Brandvain et al., } 2014\end{array}$ \\
\hline \multirow{2}{*}{ R. paraguas } & \multirow{2}{*}{-} & TG 1415 & $\begin{array}{l}\text { Colombia: Valle del } \\
\text { Cauca: El Cairo }\end{array}$ & This study \\
\hline & & TG 1480 & $\begin{array}{l}\text { Colombia: Valle del } \\
\text { Cauca: El Cairo }\end{array}$ & This study \\
\hline R. paraguayensis & R. scitula & UFMT 1876 & $\begin{array}{l}\text { Brazil: Mato Grosso: } \\
\text { Poconé }\end{array}$ & This study \\
\hline R. cf. paraguayensis & R. stanlaii & SMF 88237 & $\begin{array}{l}\text { Bolivia: Santa Cruz: } \\
\text { Ñuflo de Chavez, San } \\
\text { Sebastián }\end{array}$ & Jansen et al., 2011 \\
\hline \multirow{3}{*}{ R. poeppigii } & \multirow{3}{*}{-} & MUBI 6863 & $\begin{array}{l}\text { Peru: Cusco: Quispi- } \\
\text { canchis }\end{array}$ & This study \\
\hline & & MUBI 6864 & $\begin{array}{l}\text { Peru: Cusco: Quispi- } \\
\text { canchis }\end{array}$ & This study \\
\hline & & USNM 268824 & $\begin{array}{l}\text { Peru: Madre de Dios: } \\
\text { Puerto Maldonado }\end{array}$ & $\begin{array}{l}\text { Pramuk, 2006; Pramuk } \\
\text { et al., 2008, Brandvain } \\
\text { et al., } 2014\end{array}$ \\
\hline
\end{tabular}




\section{APPENDIX 1 continued}

\begin{tabular}{|c|c|c|c|c|}
\hline \multicolumn{5}{|l|}{ RHINELLA } \\
\hline Current taxonomy & Updated taxonomy & Voucher & Locality & Sources \\
\hline \multirow{6}{*}{ R. proboscidea } & \multirow[b]{2}{*}{-} & AMNH-FS 20085 & $\begin{array}{l}\text { Brazil: Roraima: Flo- } \\
\text { resta }\end{array}$ & This study \\
\hline & & CTGA-UFAM 5602 & $\begin{array}{l}\text { Brazil: Amazonas: } \\
\text { Universidade Federal } \\
\text { do Amazonas, near } \\
\text { Manaus }\end{array}$ & Motta et al., 2018 \\
\hline & Rhinella sp. 8 & QCAZ 28573 & $\begin{array}{l}\text { Ecuador: Sucumbios: } \\
\text { Sucumbíos: Campo } \\
\text { Vinita, vía Palma Roja- } \\
\text { Pto El Carmen de } \\
\text { Putumayo }\end{array}$ & This study \\
\hline & \multirow{3}{*}{ Rhinella sp. 9} & CORBIDI 102 & Peru: Loreto: Maynas & This study \\
\hline & & CORBIDI 5835 & Peru: Loreto: Maynas & This study \\
\hline & & MNCN-ADN 26559 & $\begin{array}{l}\text { Peru: Loreto: Maronal, } \\
\text { Río Ampiyacu }\end{array}$ & This study \\
\hline \multirow{2}{*}{ R. pygmaea } & \multirow{2}{*}{-} & CFBH 2894 & $\begin{array}{l}\text { Brazil: Rio de Janeiro: } \\
\text { Sao João da Barra }\end{array}$ & Pereyra et al., 2016a \\
\hline & & CFBH-T 15163 & $\begin{array}{l}\text { Brazil: Espírito Santo: } \\
\text { Mimoso do Sul }\end{array}$ & $\begin{array}{l}\text { This study, Pereyra et } \\
\text { al., 2016a }\end{array}$ \\
\hline R. quechua & - & MNCN-ADN 3927 & $\begin{array}{l}\text { Bolivia: Cochabamba: } \\
\text { Parque Nacional } \\
\text { Carrasco, Sehuencas }\end{array}$ & This study \\
\hline \multirow{2}{*}{ R. rubescens } & \multirow{2}{*}{-} & CFBH 5836 & $\begin{array}{l}\text { Brazil: Minas Gerais: } \\
\text { Poços de Caldas }\end{array}$ & This study \\
\hline & & CFBH 7696 & $\begin{array}{l}\text { Brazil: Goiás: Cocalz- } \\
\text { inho de Goiás }\end{array}$ & $\begin{array}{l}\text { This study; Thomé et } \\
\text { al., } 2010\end{array}$ \\
\hline R. rubropunctata & - & MACN 52275 & $\begin{array}{l}\text { Argentina: Chubut: } \\
\text { Cushamen }\end{array}$ & This study \\
\hline \multirow{2}{*}{ R. ruizi } & \multirow{2}{*}{-} & AML 39 & $\begin{array}{l}\text { Colombia: Antioquia: } \\
\text { Medellín }\end{array}$ & This study \\
\hline & & AML 40 & $\begin{array}{l}\text { Colombia: Antioquia: } \\
\text { Medellín }\end{array}$ & This study \\
\hline \multirow{2}{*}{ R. rumbolli } & \multirow{2}{*}{-} & MACN 53782 & $\begin{array}{l}\text { Argentina: Salta: Santa } \\
\text { Victoria, Parque } \\
\text { Nacional Baritú }\end{array}$ & This study \\
\hline & & MACN 43719 & $\begin{array}{l}\text { Argentina: Salta: Santa } \\
\text { Victoria, Parque } \\
\text { Nacional Baritú }\end{array}$ & This study \\
\hline R. scitula & - & IIBP 849 & Paraguay: Concepción & This study \\
\hline R. cf. scitula & R. scitula & CFBH 42359 & $\begin{array}{l}\text { Brazil: Mato Grosso do } \\
\text { Sul: Corumbá }\end{array}$ & This study \\
\hline R. sclerocephala & - & MHNLS 7495 & $\begin{array}{l}\text { Venezuela: Cojedes: } \\
\text { Cerro Azul, fila La } \\
\text { Blanquera }\end{array}$ & This study \\
\hline
\end{tabular}




\section{APPENDIX 1 continued}

\begin{tabular}{|c|c|c|c|c|}
\hline RHINELLA & & & & \\
\hline Current taxonomy & Updated taxonomy & Voucher & Locality & Sources \\
\hline \multirow{4}{*}{ R. spinulosa papillosa } & \multirow{4}{*}{ R. papillosa } & ВВ 983 & $\begin{array}{l}\text { Argentina: Neuquén: } \\
\text { Minas }\end{array}$ & This study \\
\hline & & BB 1032 & $\begin{array}{l}\text { Argentina: Rio Negro: } \\
\text { Bariloche, Pampa } \\
\text { Linda }\end{array}$ & Frost et al., 2006 \\
\hline & & MACN 49782 & $\begin{array}{l}\text { Argentina: Chubut: } \\
\text { Lago Puelo }\end{array}$ & This study \\
\hline & & NB 96-23 & $\begin{array}{l}\text { Argentina: Neuquén: } \\
\text { Laguna Blanca }\end{array}$ & $\begin{array}{l}\text { Pauly et al., 2004, } \\
\text { Brandvain et al., } 2014\end{array}$ \\
\hline \multirow{6}{*}{ R. spinulosa spinulosa } & \multirow{4}{*}{ R. spinulosa } & IDLR 3837 & $\begin{array}{l}\text { Bolivia: La Paz: stream } \\
\text { between Charazani } \\
\text { and Curva }\end{array}$ & $\begin{array}{l}\text { Pramuk, 2006; Pramuk } \\
\text { et al., } 2008\end{array}$ \\
\hline & & MUBI 10737 & Peru: Cusco: Colcha & This study \\
\hline & & MUBI 10770 & $\begin{array}{l}\text { Peru: Cusco: } \\
\text { Chumbivilcas }\end{array}$ & This study \\
\hline & & nv & Peru: Puno: Acocollo & This study \\
\hline & \multirow{2}{*}{ R. altiperuviana } & MACN 49701 & $\begin{array}{l}\text { Argentina: Jujuy: Tum- } \\
\text { baya, Quebrada de } \\
\text { Sepultura }\end{array}$ & This study \\
\hline & & MNCN 41989 & $\begin{array}{l}\text { Bolivia: La Paz: Inqui- } \\
\text { sivi, Quebrada entre } \\
\text { Quime e Inquisivi }\end{array}$ & This study \\
\hline \multirow[t]{2}{*}{ R. spinulosa trifolium } & \multirow[t]{2}{*}{ R. trifolium } & CORBIDI 5530 & $\begin{array}{l}\text { Peru: Lima: Huancaya, } \\
\text { Vilca }\end{array}$ & This study \\
\hline & & nv & Peru: Junín: Huancayo & This study \\
\hline \multirow{3}{*}{ R. stanlaii } & \multirow{3}{*}{-} & ZUFG 6456 & $\begin{array}{l}\text { Brazil: Mato Grosso: } \\
\text { Tangará da Serra }\end{array}$ & This study \\
\hline & & MNCN-ADN 4160 & $\begin{array}{l}\text { Bolivia: Santa Cruz: } \\
\text { Amboró, Ichilo }\end{array}$ & This study \\
\hline & & MNCN-ADN 6274 & $\begin{array}{l}\text { Bolivia: Cochabamba: } \\
\text { Carrasco, Chaquisacha }\end{array}$ & This study \\
\hline R. aff. stanlaii & Rhinella sp. 15 & MNCN-ADN 4159 & $\begin{array}{l}\text { Bolivia: La Paz: Parque } \\
\text { Nacional Madidi, Ser- } \\
\text { ranía Sadiri }\end{array}$ & This study \\
\hline R. sternosignata & - & nv & $\begin{array}{l}\text { Venezuela: Barinas: } \\
\text { Cano Los Monos, } \\
\text { Acequias }\end{array}$ & Pereyra et al., 2016a \\
\hline \multirow{2}{*}{ R. cf. sternosignata } & \multirow{2}{*}{ Rhinella sp. 13} & MAR 1314 & $\begin{array}{l}\text { Colombia: Boyacá: } \\
\text { Pajarito }\end{array}$ & This study \\
\hline & & MAR 1955 & $\begin{array}{l}\text { Colombia: Caquetá: } \\
\text { Florencia }\end{array}$ & This study \\
\hline
\end{tabular}


APPENDIX 1 continued

\begin{tabular}{|c|c|c|c|c|}
\hline \multicolumn{5}{|l|}{ RHINELLA } \\
\hline Current taxonomy & Updated taxonomy & Voucher & Locality & Sources \\
\hline \multirow{5}{*}{ R. tacana } & \multirow{5}{*}{-} & MUBI 6950 & $\begin{array}{l}\text { Peru: Cusco: Quispi- } \\
\text { canchis }\end{array}$ & This study \\
\hline & & MUBI 7409 & $\begin{array}{l}\text { Peru: Cusco: Quispi- } \\
\text { canchis }\end{array}$ & This study \\
\hline & & MUBI 7007 & $\begin{array}{l}\text { Peru: Cusco: Quispi- } \\
\text { canchis, Camanti }\end{array}$ & This study \\
\hline & & MNK 7187 & $\begin{array}{l}\text { Bolivia: La Paz: Parque } \\
\text { Nacional Madidi }\end{array}$ & This study \\
\hline & & UTA 53310 & Bolivia: La Paz & $\begin{array}{l}\text { Pramuk, 2006; Pramuk } \\
\text { et al., } 2008\end{array}$ \\
\hline \multirow{2}{*}{ R. tenrec } & \multirow{2}{*}{-} & MAR 3584 & $\begin{array}{l}\text { Colombia: Antioquia: } \\
\text { Parque Nacional Las } \\
\text { Orquídeas }\end{array}$ & This study \\
\hline & & MAR 3585 & $\begin{array}{l}\text { Colombia: Antioquia: } \\
\text { Parque Nacional Las } \\
\text { Orquídeas }\end{array}$ & This study \\
\hline \multirow{4}{*}{ R. vellardi } & \multirow{4}{*}{-} & CORBIDI 7626 & $\begin{array}{l}\text { Peru: La Libertad: } \\
\text { Pataz, Vijus }\end{array}$ & This study \\
\hline & & KU 211765 & $\begin{array}{l}\text { Peru: Cajamarca: Caja- } \\
\text { bamba }\end{array}$ & $\begin{array}{l}\text { Pramuk, 2006; Pramuk } \\
\text { et al., } 2008\end{array}$ \\
\hline & & MUBI 14281 & $\begin{array}{l}\text { Peru: La Libertad: } \\
\text { Sánchez Carrion }\end{array}$ & This study \\
\hline & & MUBI 14291 & $\begin{array}{l}\text { Peru: La Libertad: } \\
\text { Sánchez Carrion }\end{array}$ & This study \\
\hline \multirow{4}{*}{ R. veraguensis } & \multirow{4}{*}{-} & IDLR 3820 & Bolivia: La Paz & $\begin{array}{l}\text { Pramuk, 2006; Pramuk } \\
\text { et al., } 2008\end{array}$ \\
\hline & & MNCN-ADN 5808 & $\begin{array}{l}\text { Bolivia: Cochabamba: } \\
\text { Parque Nacional } \\
\text { Carrasco, Kharahuasi }\end{array}$ & This study \\
\hline & & MUBI 5946 & $\begin{array}{l}\text { Peru: Puno: Santo } \\
\text { Domingo }\end{array}$ & This study \\
\hline & & USNM 346048 & $\begin{array}{l}\text { Peru: Cusco: Paucart- } \\
\text { ambo }\end{array}$ & $\begin{array}{l}\text { Pramuk, 2006; Pramuk } \\
\text { et al., } 2008\end{array}$ \\
\hline \multirow{2}{*}{$R$. veredas } & \multirow{2}{*}{-} & CFBH 20516 & $\begin{array}{l}\text { Brazil: Bahia: São } \\
\text { Desidério }\end{array}$ & This study \\
\hline & & CHUNB 44609 & $\begin{array}{l}\text { Brazil: Minas Gerais: } \\
\text { Buritizeiro }\end{array}$ & $\begin{array}{l}\text { This study; Maciel et } \\
\text { al., } 2010\end{array}$ \\
\hline \multirow{3}{*}{ R. yanachaga } & \multirow{3}{*}{-} & CORBIDI 7269 & $\begin{array}{l}\text { Peru: Pasco: Oxa- } \\
\text { pampa }\end{array}$ & This study \\
\hline & & MUBI 7119 & $\begin{array}{l}\text { Peru: Pasco: Oxa- } \\
\text { pampa }\end{array}$ & This study \\
\hline & & MUBI 7121 & $\begin{array}{l}\text { Peru: Pasco: Oxa- } \\
\text { pampa }\end{array}$ & This study \\
\hline
\end{tabular}


APPENDIX 1 continued

\begin{tabular}{|c|c|c|c|c|}
\hline RHINELLA & & & & \\
\hline Current taxonomy & Updated taxonomy & Voucher & Locality & Sources \\
\hline \multirow{4}{*}{ R. yunga } & \multirow{4}{*}{ R. iserni } & NMP6V 75552 & $\begin{array}{l}\text { Peru: Junín: Chan- } \\
\text { chamayo, Bosque de } \\
\text { Protección Pui Pui }\end{array}$ & $\begin{array}{l}\text { This study; Cusi et al., } \\
2017\end{array}$ \\
\hline & & MUSM 31096 & $\begin{array}{l}\text { Peru: Cusco: Oxa- } \\
\text { pampa, Quebrada San } \\
\text { Alberto, Parque Nacio- } \\
\text { nal Yanachaga-Chemi- } \\
\text { llén }\end{array}$ & This study \\
\hline & & MUSM 31097 & $\begin{array}{l}\text { Peru: Cusco: Oxa- } \\
\text { pampa, Quebrada San } \\
\text { Alberto, Parque Nacio- } \\
\text { nal Yanachaga-Chemi- } \\
\text { llén }\end{array}$ & This study \\
\hline & & MUSM 31950 & $\begin{array}{l}\text { Peru: Junín: Chan- } \\
\text { chamayo, Bosque de } \\
\text { Protección Pui Pui }\end{array}$ & This study \\
\hline R. sp. "gr. acrolopha" & Rhinella sp. 4 & TG 2115 & $\begin{array}{l}\text { Colombia: Tolima: } \\
\text { Murillo, km } 22 \text { carret- } \\
\text { era Murillo-Manizales }\end{array}$ & Machado et al., 2016 \\
\hline \multirow{5}{*}{ R. sp. gr. margaritifera } & \multirow{2}{*}{ R. dapsilis } & MC 204 & French Guiana: Saul & Fouquet et al., 2007c \\
\hline & & PG 144 & French Guiana: Patawa & Fouquet et al., 2007c \\
\hline & \multirow{2}{*}{ Rhinella sp. 5} & QCAZ 53072 & $\begin{array}{l}\text { Ecuador: Pastaza: } \\
\text { Comunidad Puka Yaku }\end{array}$ & This study \\
\hline & & QCAZ 53142 & $\begin{array}{l}\text { Ecuador: Pastaza: } \\
\text { Comunidad Campus }\end{array}$ & This study \\
\hline & Rhinella sp. 13 & MHNLS 21837 & $\begin{array}{l}\text { Venezuela: Miranda: } \\
\text { Río Araira }\end{array}$ & This study \\
\hline R. sp. gr. marina & "R. icterica" & LGE 19195 & $\begin{array}{l}\text { Argentina: Misiones: } \\
\text { Posadas }\end{array}$ & This study \\
\hline
\end{tabular}

\begin{tabular}{llll}
\hline OUTGROUPS & Voucher & Locality & Sources \\
\hline Amazophrynella aff. minuta & MJH 7095 & $\begin{array}{l}\text { Peru: Huanuco: Rio Llullapi- } \\
\text { chis, Panguana }\end{array}$ & Faivovich et al., 2005; Grant et al., 2006 \\
\hline Anaxyrus americanus & CAS 207258 & U.S.: Mississippi: Boswell Lake & $\begin{array}{l}\text { van Bocxlaer et al., 2009; Liedtke et al., } \\
\text { 2016 }\end{array}$ \\
\hline Anaxyrus boreas & CAS 201586 & $\begin{array}{l}\text { U.S.: California: Grover Hot } \\
\text { Springs Rd }\end{array}$ & $\begin{array}{l}\text { van Bocxlaer et al., 2009; Liedtke et al., } \\
\text { 2016 }\end{array}$ \\
\hline Anaxyrus quercicus & MVZ 223370 & U.S.: Florida: Charlotte & Pauly et al., 2004; Brandvain et al., 2014 \\
\hline Anaxyrus woodhousii & KU 224658 & U.S.: Kansas: Barber, Sharon & Pramuk, 2006; Pramuk et al., 2008 \\
\hline Ansonia longidigita & VUB 666 & Malaysia: Borneo & $\begin{array}{l}\text { van Bocxlaer et al 2009; Liedtke et al } \\
\text { 2016 }\end{array}$ \\
\hline
\end{tabular}


APPENDIX 1 continued

\begin{tabular}{|c|c|c|c|}
\hline OUTGROUPS & Voucher & Locality & Sources \\
\hline \multirow{2}{*}{ Bufo bufo } & VUB 982 & Belgium & van Bocxlaer et al., 2009 \\
\hline & MVZ 230209 & Turkey: Bursa: Bursa & Pramuk, 2006; Pramuk et al., 2008 \\
\hline \multirow{2}{*}{ Bufo gargarizans } & CAS 228184 & $\begin{array}{l}\text { China: Yunnan: Fugong- } \\
\text { Gongshan rd, N of Fugong }\end{array}$ & $\begin{array}{l}\text { van Bocxlaer et al., 2009; Liedtke et al., } \\
2016\end{array}$ \\
\hline & USNM 292081 & China: Sichuan: Shimian & Pramuk, 2006; Pramuk et al., 2008 \\
\hline Bufotes luristanicus & NP 13-1 & nd & van Bocxlaer et al., 2010 \\
\hline $\begin{array}{l}\text { Duttaphrynus } \\
\text { melanostictus }\end{array}$ & VUB 52 & India & $\begin{array}{l}\text { Bossuyt and Milinkovitch, 2000; Biju and } \\
\text { Bossuyt, 2003; Roelants et al., 2007; van } \\
\text { Bocxlaer et al., } 2009\end{array}$ \\
\hline \multirow{2}{*}{ Incilius alvarius } & USNM 320001 & U.S.: Arizona: Continental & Pramuk, 2006; Pramuk et al., 2008 \\
\hline & TWR 628 & U.S.: Arizona: Pima, Why & Wiens et al., 2005 \\
\hline Incilius coniferus & KU 217480 & $\begin{array}{l}\text { Ecuador: Pichincha: Vicente } \\
\text { Maldonado }\end{array}$ & Pramuk, 2006; Pramuk et al., 2008 \\
\hline \multirow[t]{2}{*}{ Incilius nebulifer } & UTA 52489 & U.S.: Louisiana: Tangipahoa & $\begin{array}{l}\text { Mulcahy and Mendelson, 2000; Mendel- } \\
\text { son et al., } 2011\end{array}$ \\
\hline & DCC 3170 & U.S.: Texas: Gulf Coast & Santos and Cannatella, 2011 \\
\hline Incilius valliceps & UTA 13097 & Mexico: Chiapas & $\begin{array}{l}\text { Mulcahy and Mendelson, 2000, Pauly et } \\
\text { al., 2004; Brandvain et al., } 2014\end{array}$ \\
\hline Ingerophrynus galeatus & FMNH 256443 & $\begin{array}{l}\text { Lao PDR: } \\
\text { Khammouan: Nakai }\end{array}$ & Pramuk, 2006; Pramuk et al., 2008 \\
\hline Leptophryne borbonica & VUB 673 & Malaysia & $\begin{array}{l}\text { Roelants et al., 2007; van Bocxlaer et al., } \\
\text { 2009; Liedtke et al., } 2016\end{array}$ \\
\hline Melanophryniscus stelzneri & VUB 985 & nd & $\begin{array}{l}\text { Roelants et al., 2007; van Bocxlaer et al., } \\
\text { 2009; Liedtke et al., } 2016\end{array}$ \\
\hline \multirow{2}{*}{ Mertensophryne loveridgei } & KMH 26653 & Tanzania & van Bocxlaer et al., 2009 \\
\hline & MCZ 32084 & Tanzania & Liedtke et al., 2016 \\
\hline Nannophryne variegata & IZUA 3198 & $\begin{array}{l}\text { Chile: XII Region: Puerto } \\
\text { Edén, Isla Wellington }\end{array}$ & Pramuk, 2006; Pramuk et al., 2008 \\
\hline \multirow[b]{2}{*}{ Nectophrynoides tornieri } & MW 1822 & Tanzania & van Bocxlaer et al., 2009 \\
\hline & $\begin{array}{l}\mathrm{BMNH} \\
2005.1375\end{array}$ & Tanzania & Roelants et al., 2007 \\
\hline Pelophryne misera & VUB 641 & Malaysia: Borneo & $\begin{array}{l}\text { van Bocxlaer et al., 2009; Liedtke et al., } \\
2016\end{array}$ \\
\hline \multirow{2}{*}{ Peltophryne empusa } & $\mathrm{nv}$ & $\begin{array}{l}\text { Cuba: Isla de la Juventud: Los } \\
\text { Indios }\end{array}$ & Alonso et al., 2012 \\
\hline & SBH 193517 & $\begin{array}{l}\text { Cuba: Granma: Bartolome } \\
\text { Maso }\end{array}$ & $\begin{array}{l}\text { Pramuk et al., 2001; Landestoy et al., } \\
2018\end{array}$ \\
\hline \multirow{2}{*}{ Peltophryne lemur } & AG nd & Puerto Rico & Pramuk, 2006; Pramuk et al., 2008 \\
\hline & SBH 190657 & Puerto Rico & Pramuk et al., 2001 \\
\hline Phrynoidis juxtaspera & VUB 649 & Malaysia: Borneo & $\begin{array}{l}\text { van Bocxlaer et al., 2009, Liedtke et al., } \\
2016\end{array}$ \\
\hline
\end{tabular}


APPENDIX 1 continued

\begin{tabular}{|c|c|c|c|}
\hline OUTGROUPS & Voucher & Locality & Sources \\
\hline Rentapia hosii & VUB 661 & Malaysia & $\begin{array}{l}\text { Roelants et al., 2007; van Bocxlaer et al., } \\
\text { 2009; Liedtke et al., } 2016\end{array}$ \\
\hline Rhaebo ecuadorensis & QCAZ 13234 & Ecuador: Napo: Talag Alto & Pramuk, 2006; Pramuk et al., 2008 \\
\hline Rhaebo guttatus & LSUMZ 17418 & $\begin{array}{l}\text { Brazil: Rondônia: Parque } \\
\text { Estadual Guajara-Mirim }\end{array}$ & Pramuk, 2006; Pramuk et al., 2008 \\
\hline Rhaebo nasicus & ROM 20650 & $\begin{array}{l}\text { Guyana: Mazaruni-Potaro: } \\
\text { Tukeit }\end{array}$ & Pramuk, 2006; Pramuk et al., 2008 \\
\hline Schismaderma carens & MW 4279 & Tanzania & van Bocxlaer et al., 2009 \\
\hline \multirow[b]{2}{*}{ Sclerophrys brauni } & MW 3840 & Tanzania & Van Bocxlaer et al., 2009 \\
\hline & $\begin{array}{l}\text { BMNH } \\
2002.350\end{array}$ & Tanzania & Roelants et al., 2007; Liedtke et al., 2016 \\
\hline Sclerophrys garmani & CAS 214829 & $\begin{array}{l}\text { Kenya: Coast Province: } \\
\text { Watamu }\end{array}$ & $\begin{array}{l}\text { Pramuk, 2006; Pramuk et al., 2008; van } \\
\text { Bocxlaer et al., 2009; Brandvain et al., } \\
2014\end{array}$ \\
\hline \multirow{2}{*}{ Sclerophrys mauritanica } & NP 22-1 & Morocco & van Bocxlaer et al., 2009 \\
\hline & VG 7-025 & Morocco & Liedtke et al., 2016 \\
\hline Sclerophrys regularis & KU 290435 & $\begin{array}{l}\text { Ghana: Eastern Region: Win- } \\
\text { neba }\end{array}$ & Pramuk, 2006; Pramuk et al., 2008 \\
\hline Sclerophrys steindachneri & CAS 214839 & $\begin{array}{l}\text { Kenya: Coast: Kilifi, Karara- } \\
\text { cha pond }\end{array}$ & $\begin{array}{l}\text { Pramuk, 2006; Pramuk et al., 2008; van } \\
\text { Bocxlaer et al., 2009; Liedtke et al., } 2016\end{array}$ \\
\hline \multirow[t]{2}{*}{ Vandijkophrynus robinsoni } & CAS 193549 & $\begin{array}{l}\text { South Africa: Northern Cape: } \\
\text { Richtersveld National Park }\end{array}$ & van Bocxlaer et al., 2010 \\
\hline & AACRG 68 & nd & Liedtke et al., 2016 \\
\hline \multirow{2}{*}{ Wolterstorffina parvipalmata } & DPL 5101 & Cameroon & Frost et al., 2006 \\
\hline & MTSN 5895 & Cameroon & van Bocxlaer et al., 2009 \\
\hline
\end{tabular}




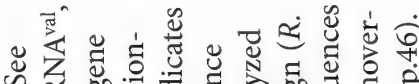

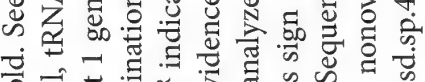

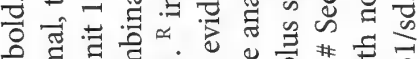

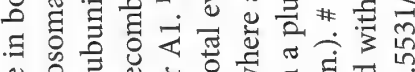

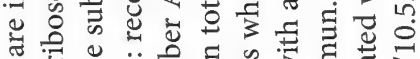

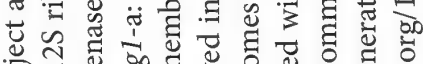
然

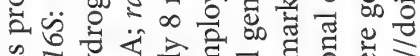

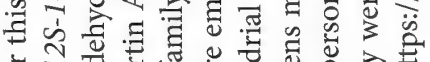

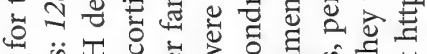
는 प्र 을 는

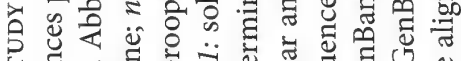

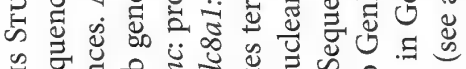

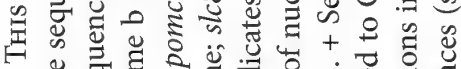

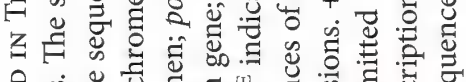

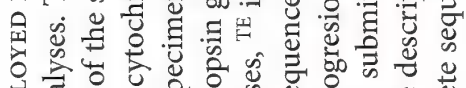

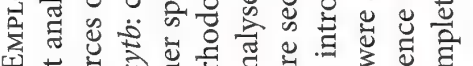
त 4 界言言

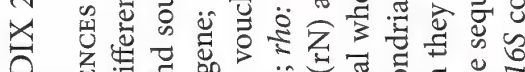

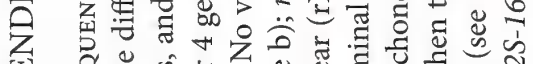

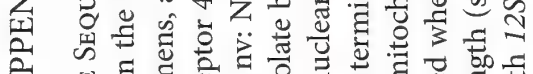

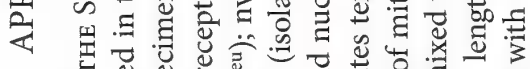

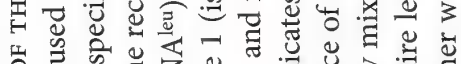

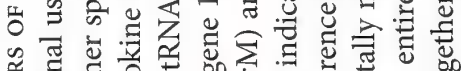

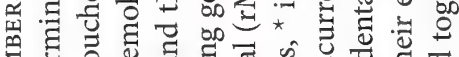

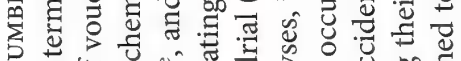
5

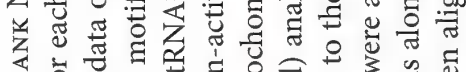

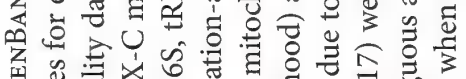

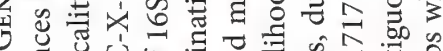

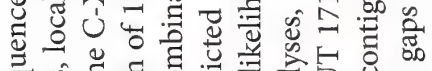

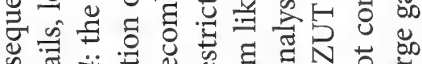

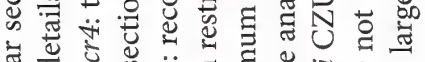

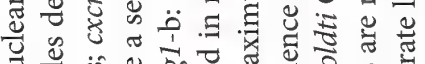

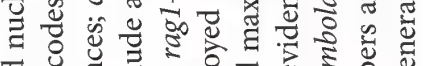

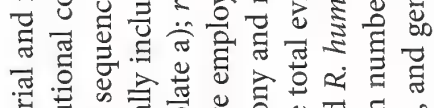

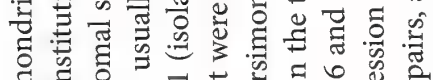

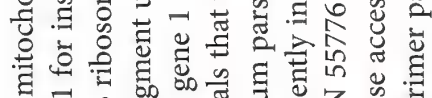

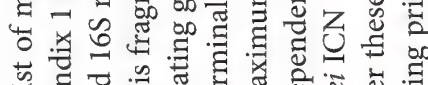

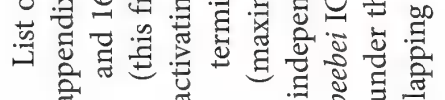

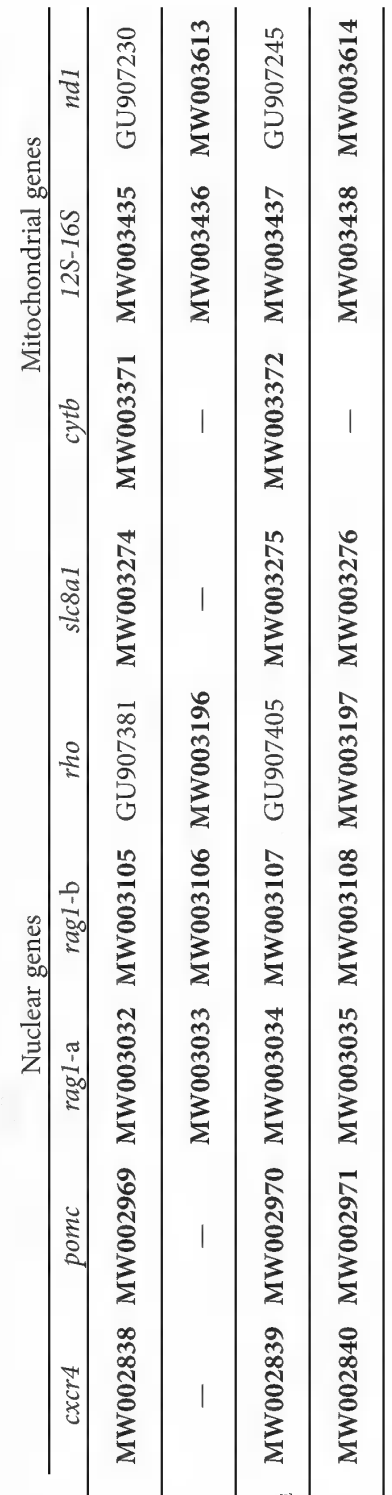

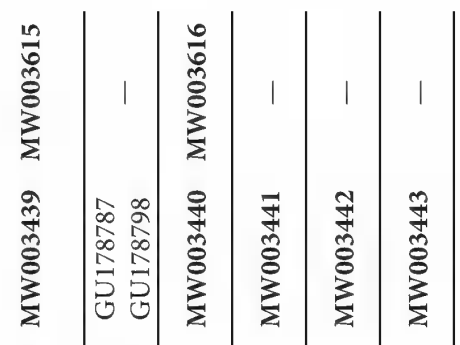

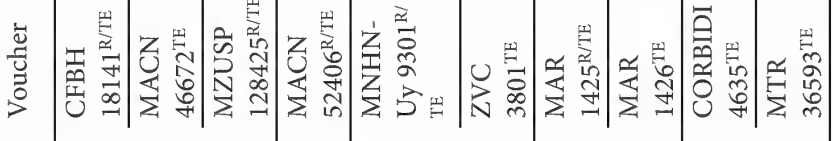

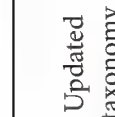

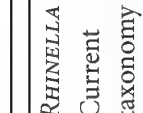
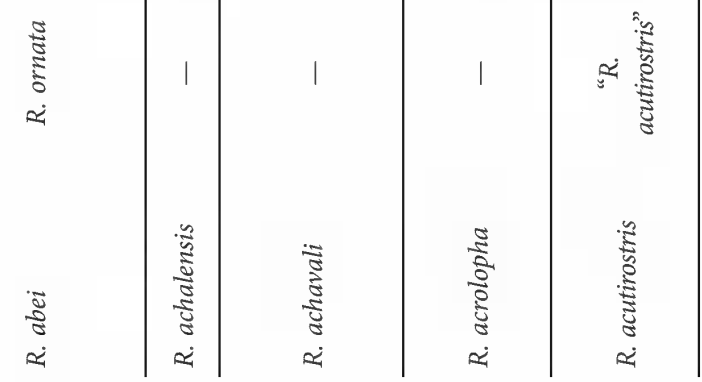


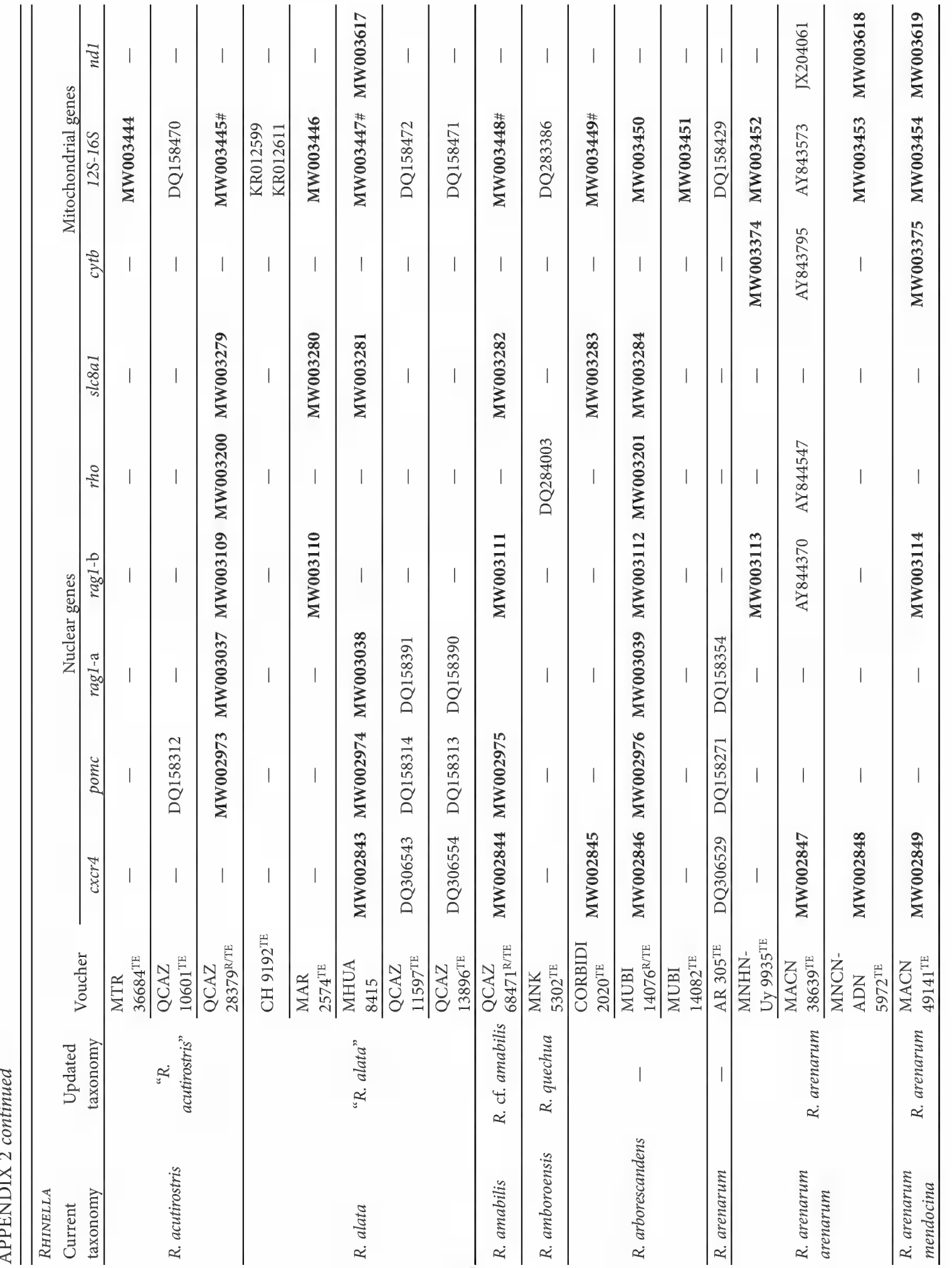




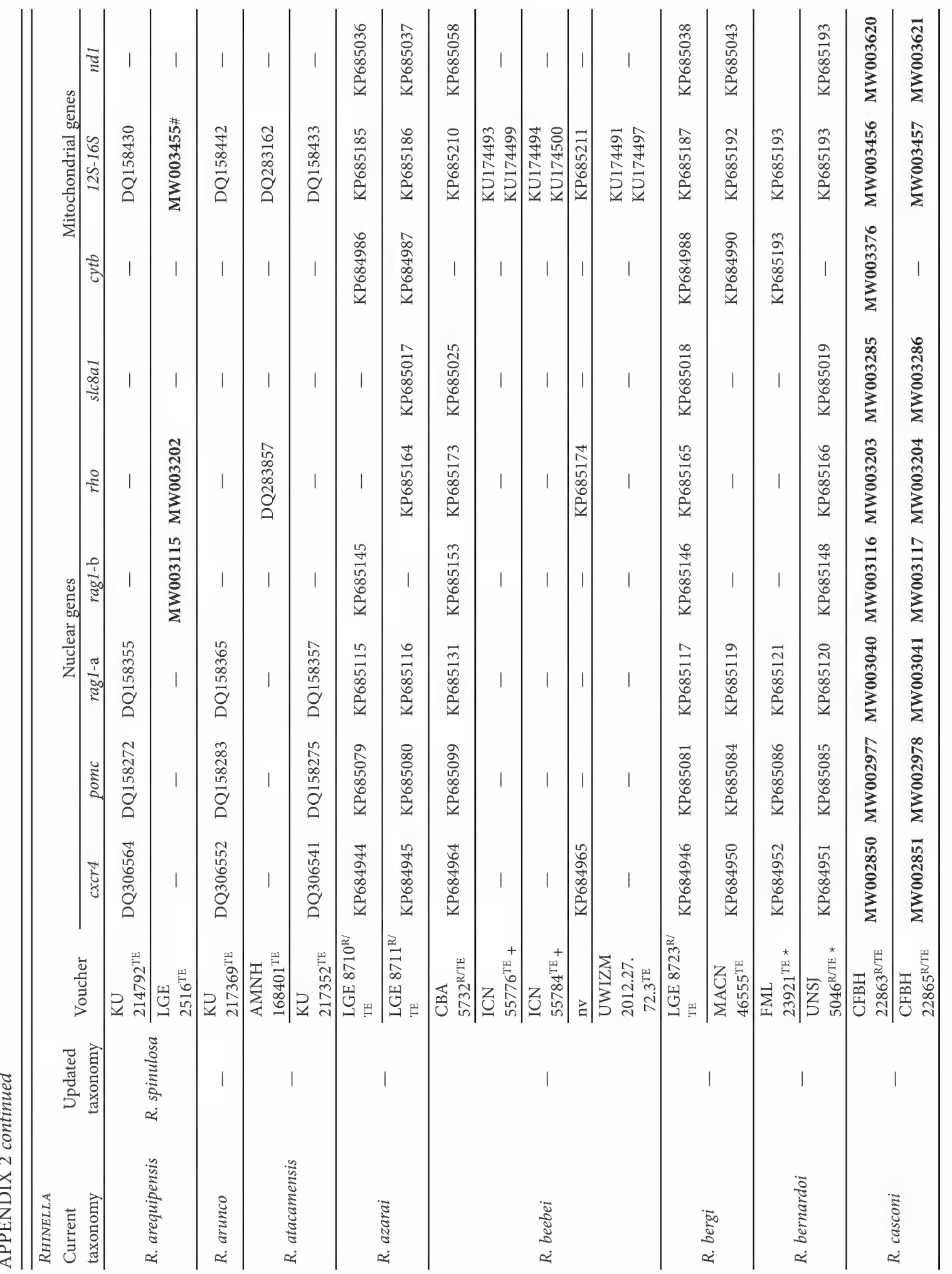



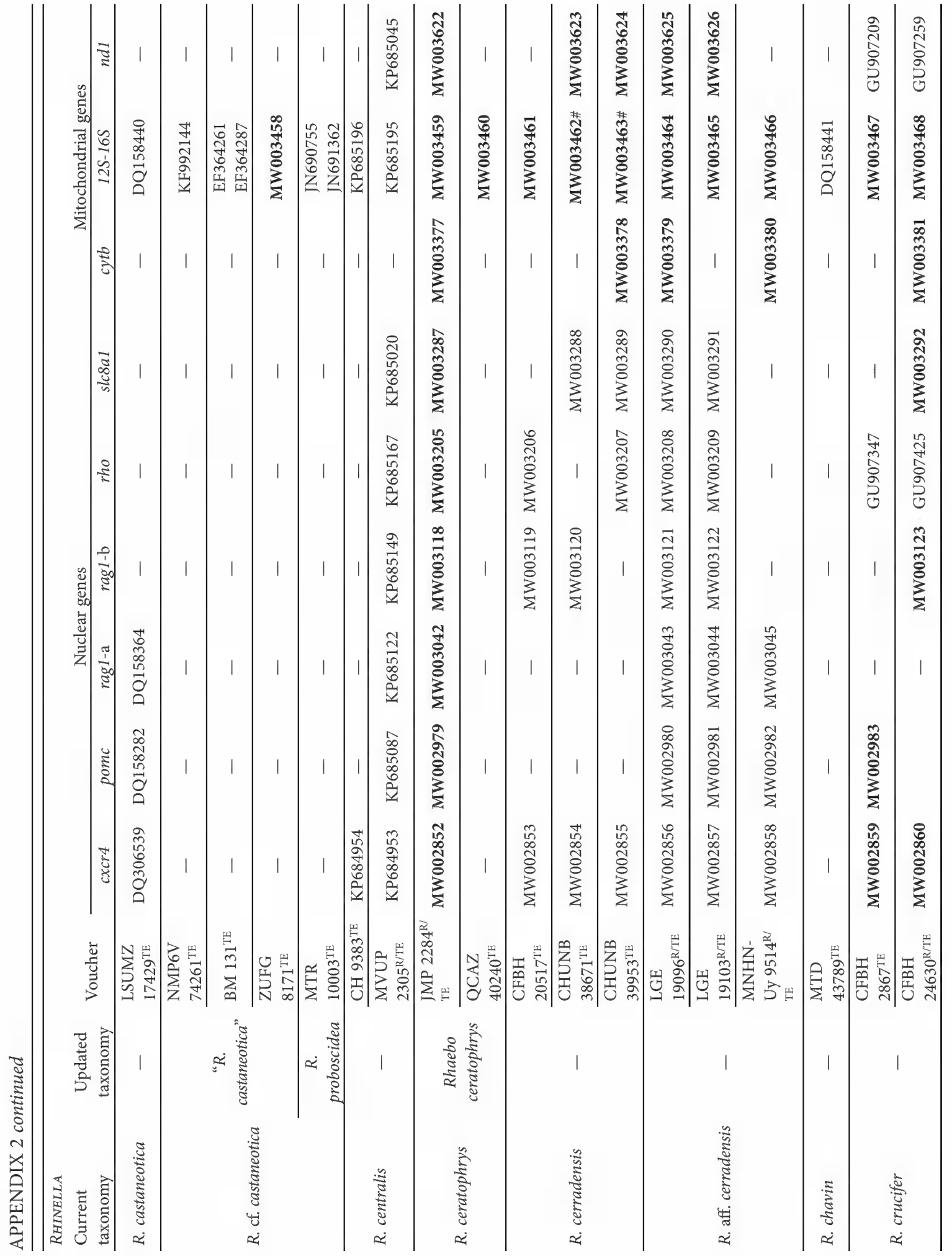


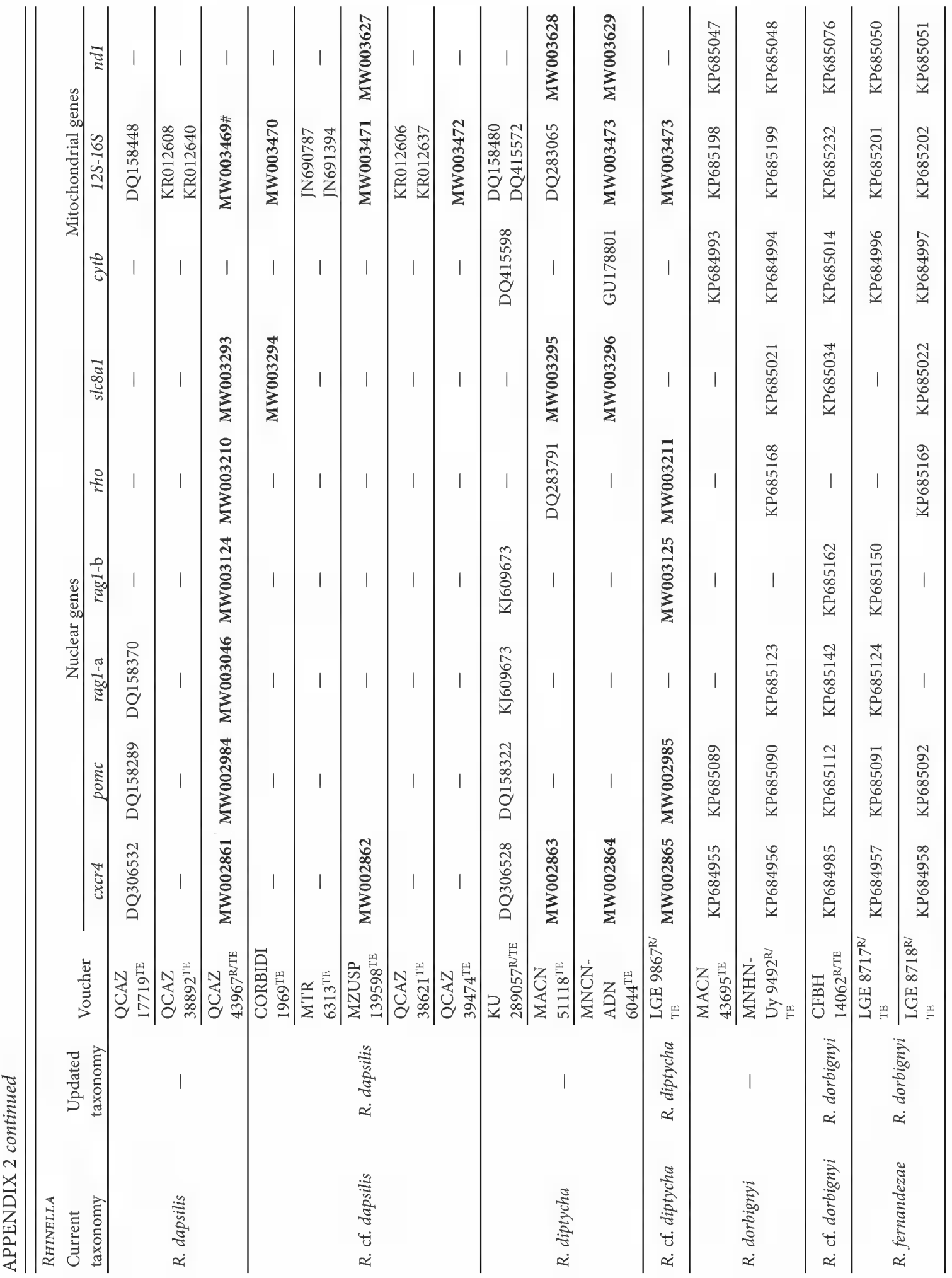




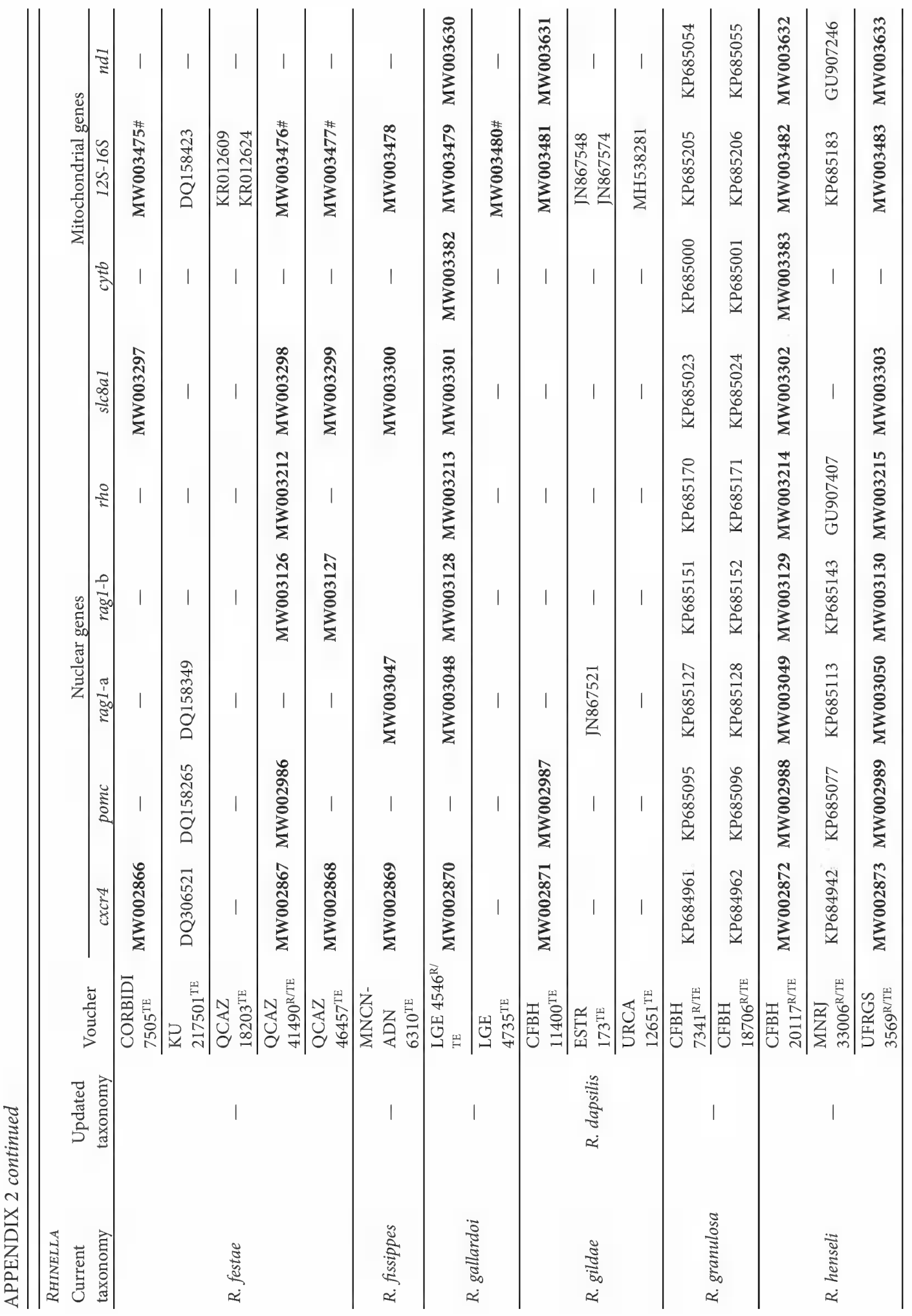




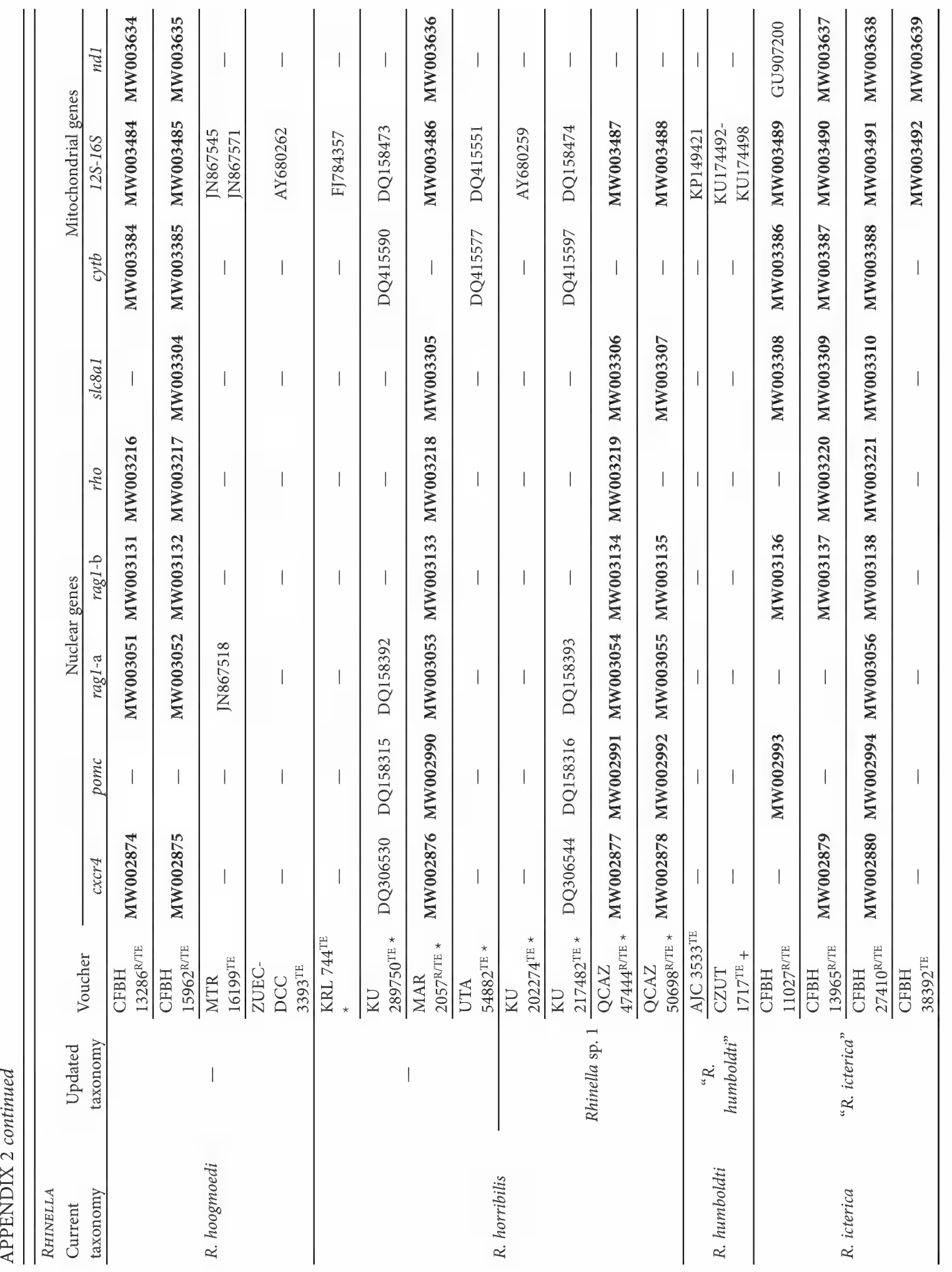




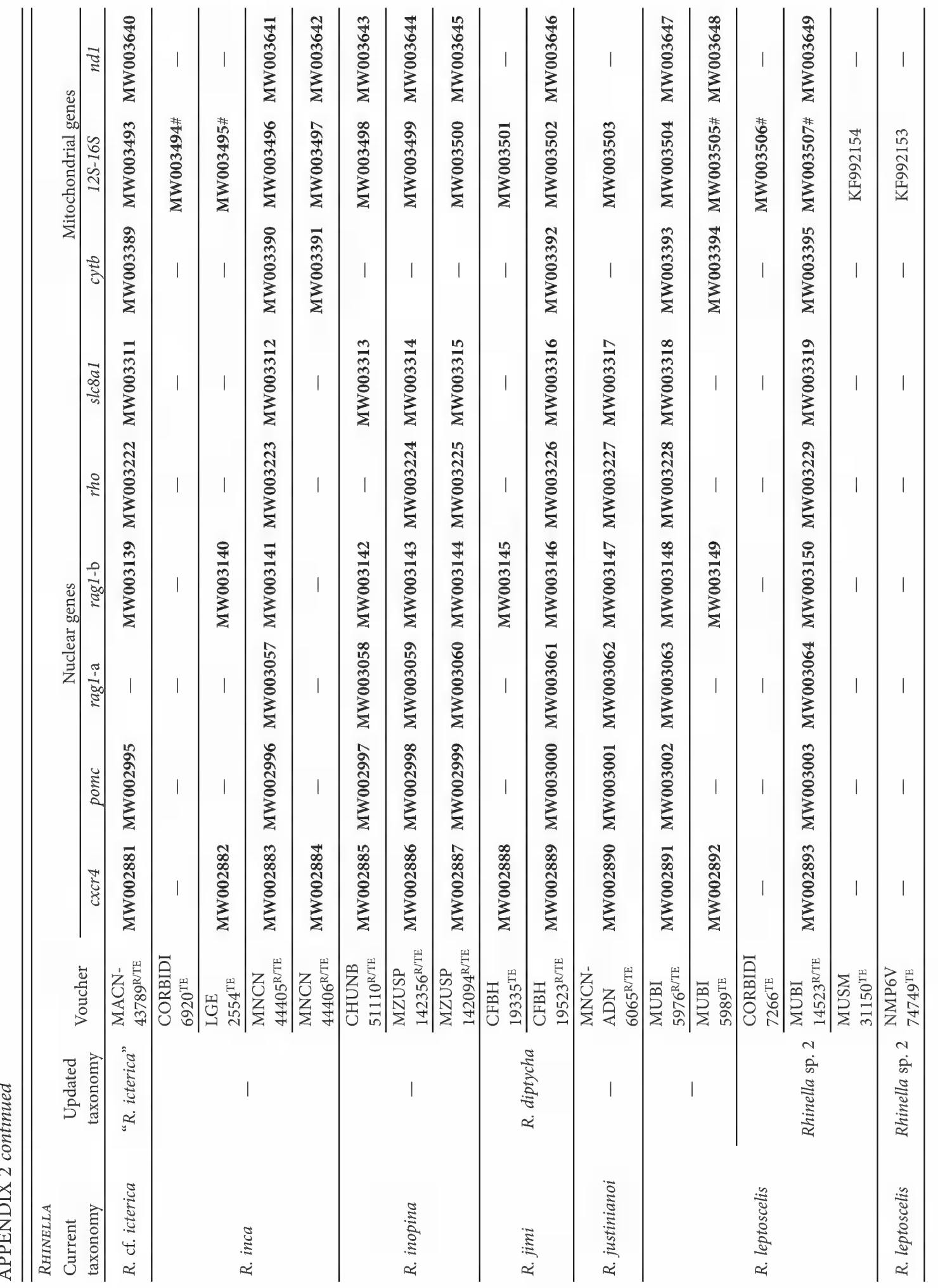




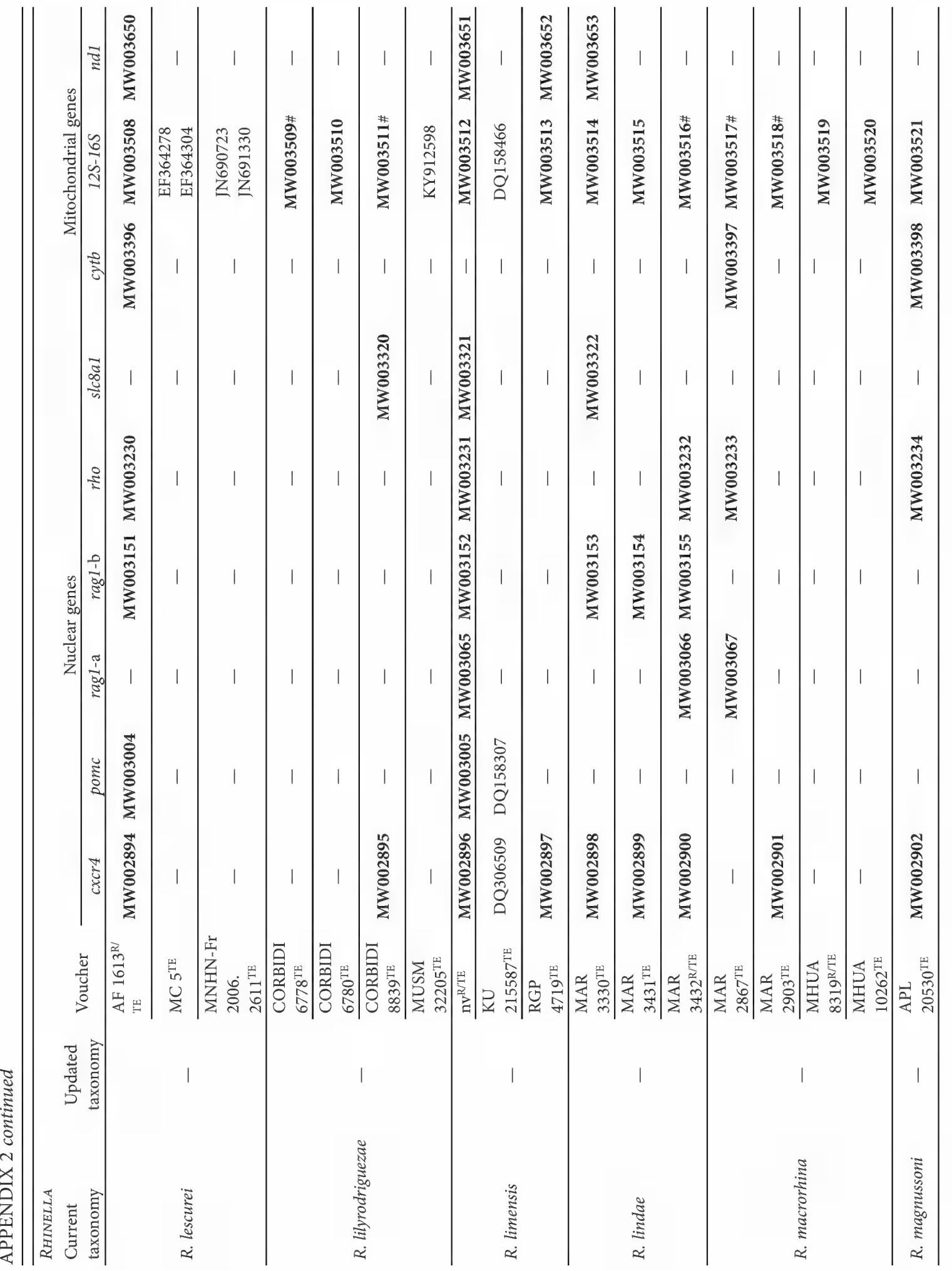




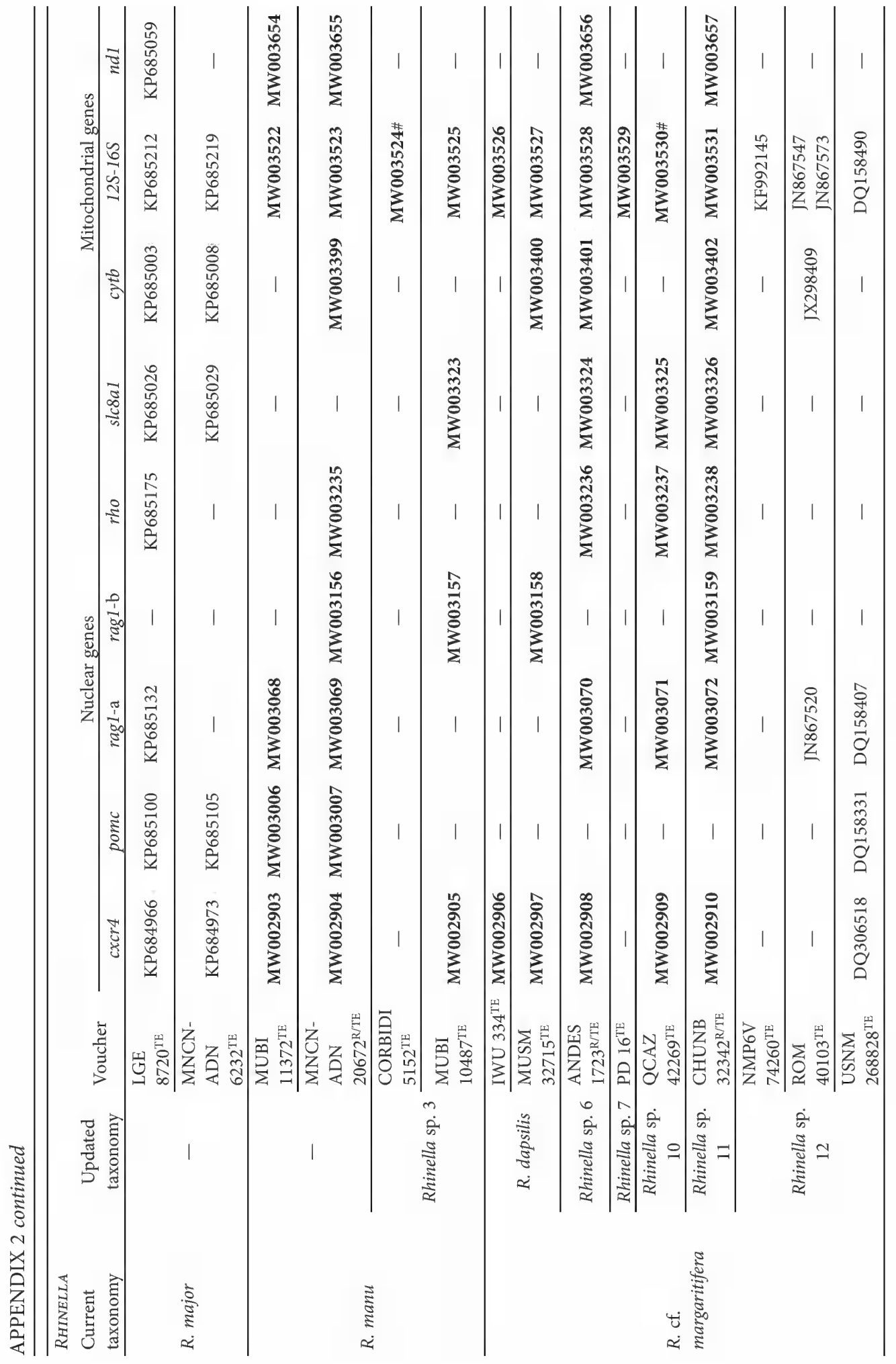




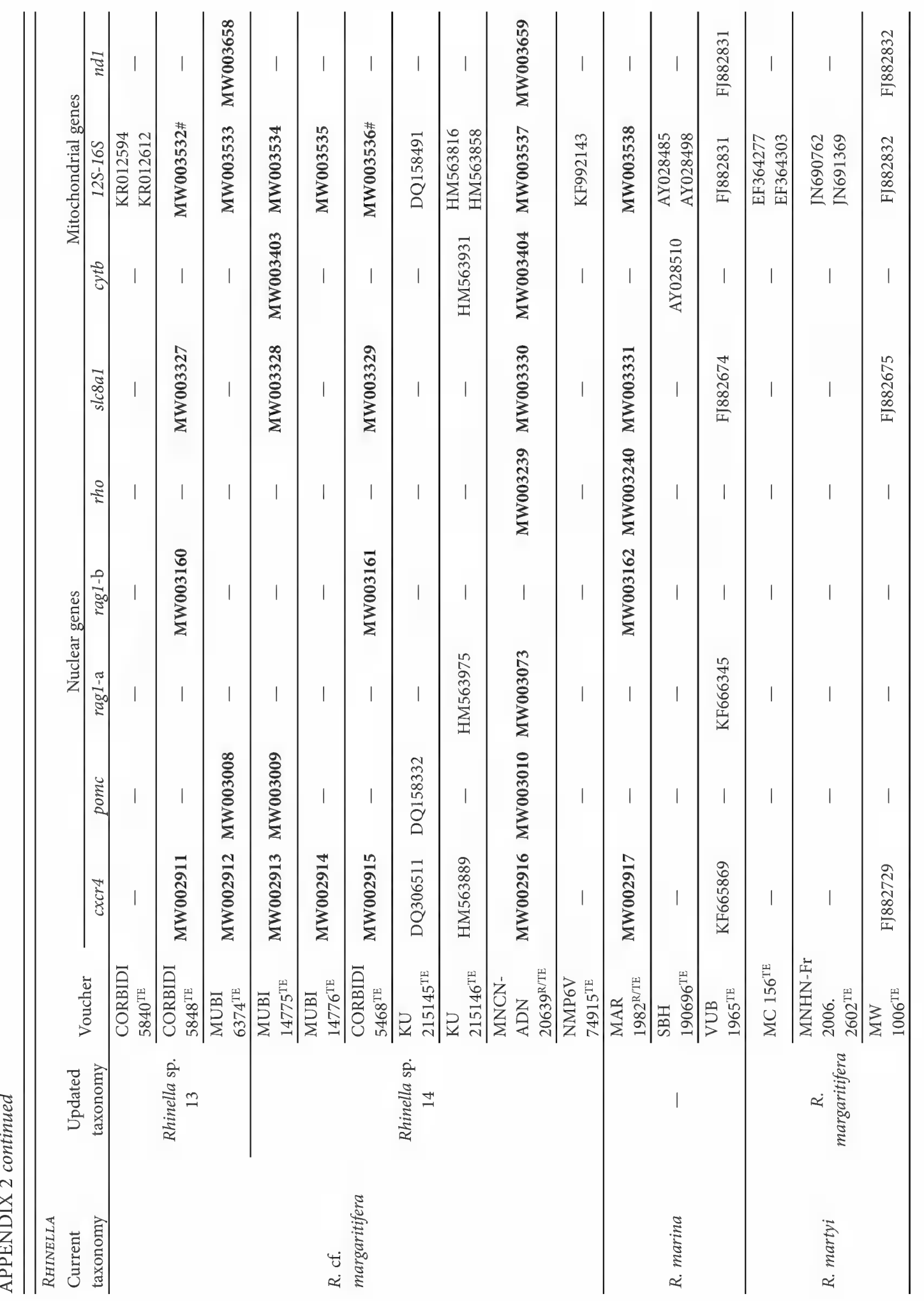




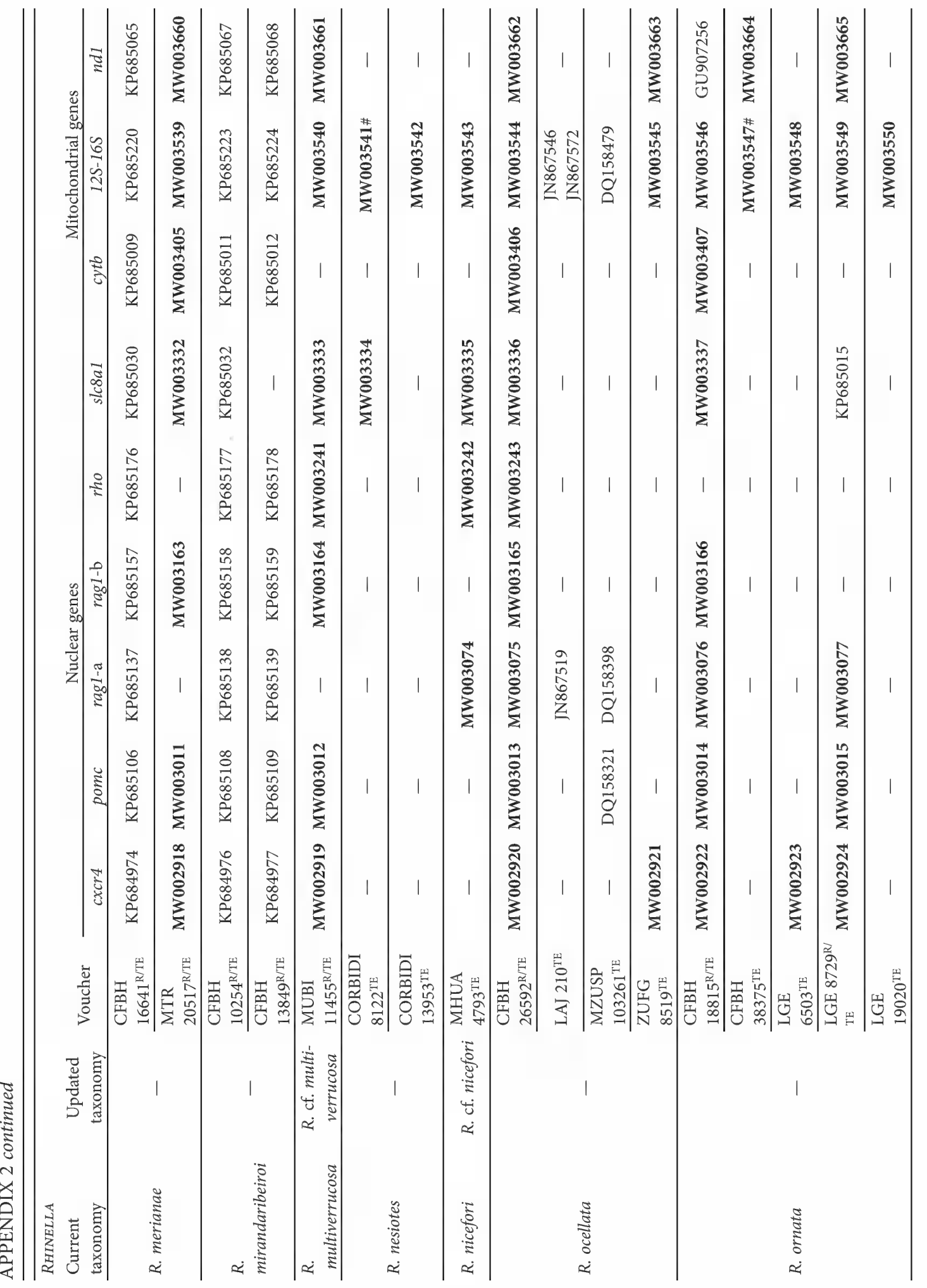




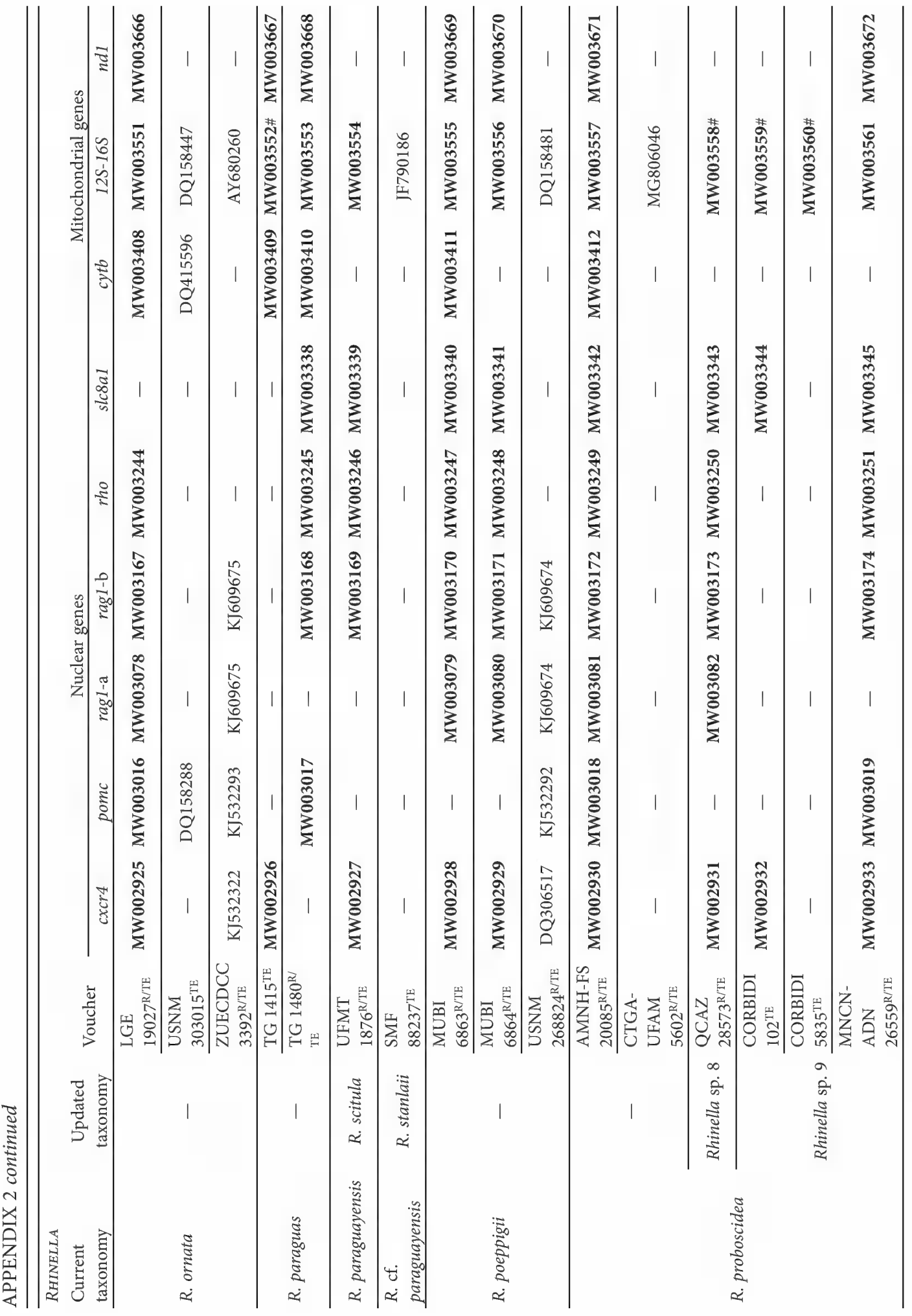




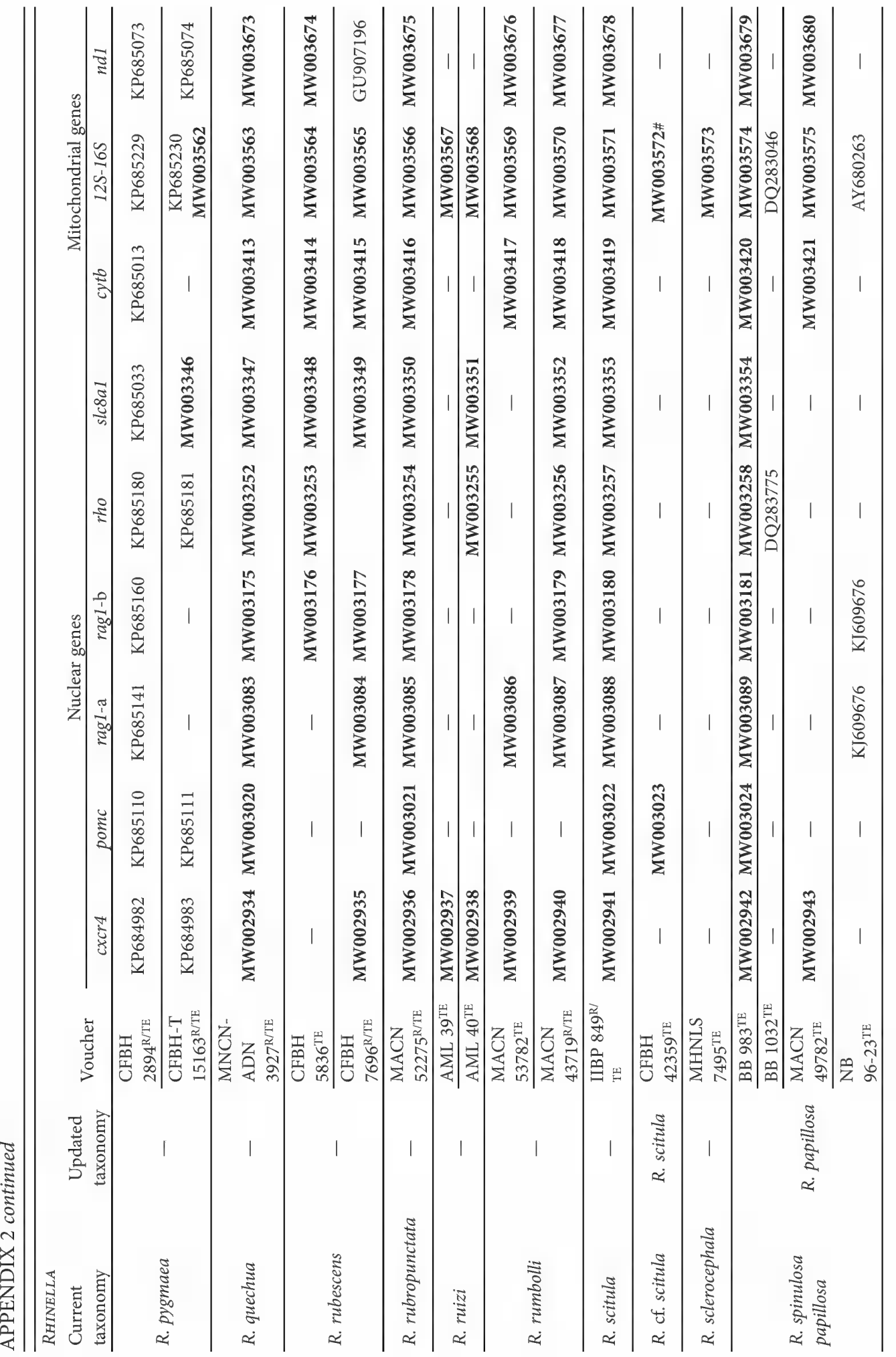




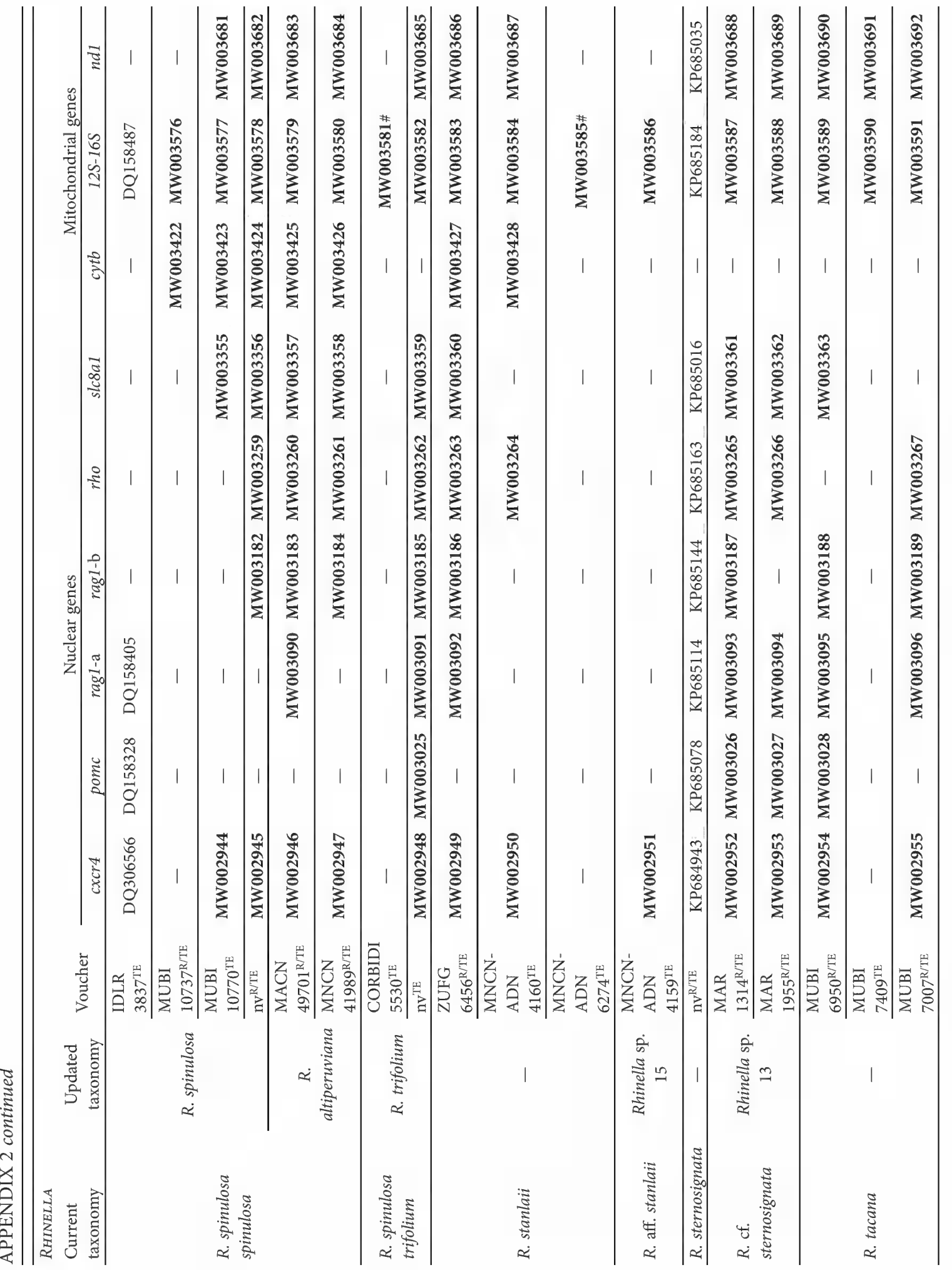




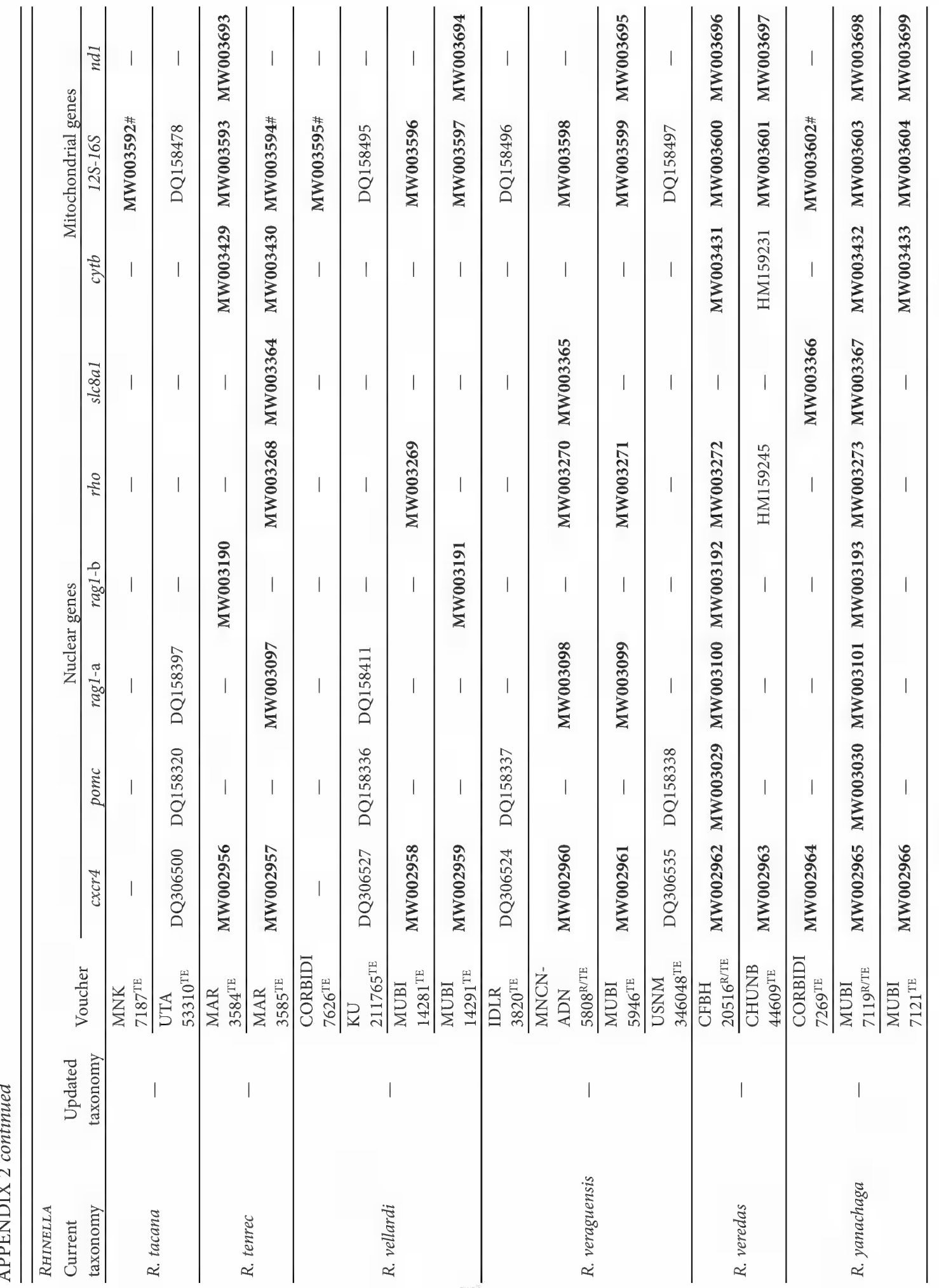




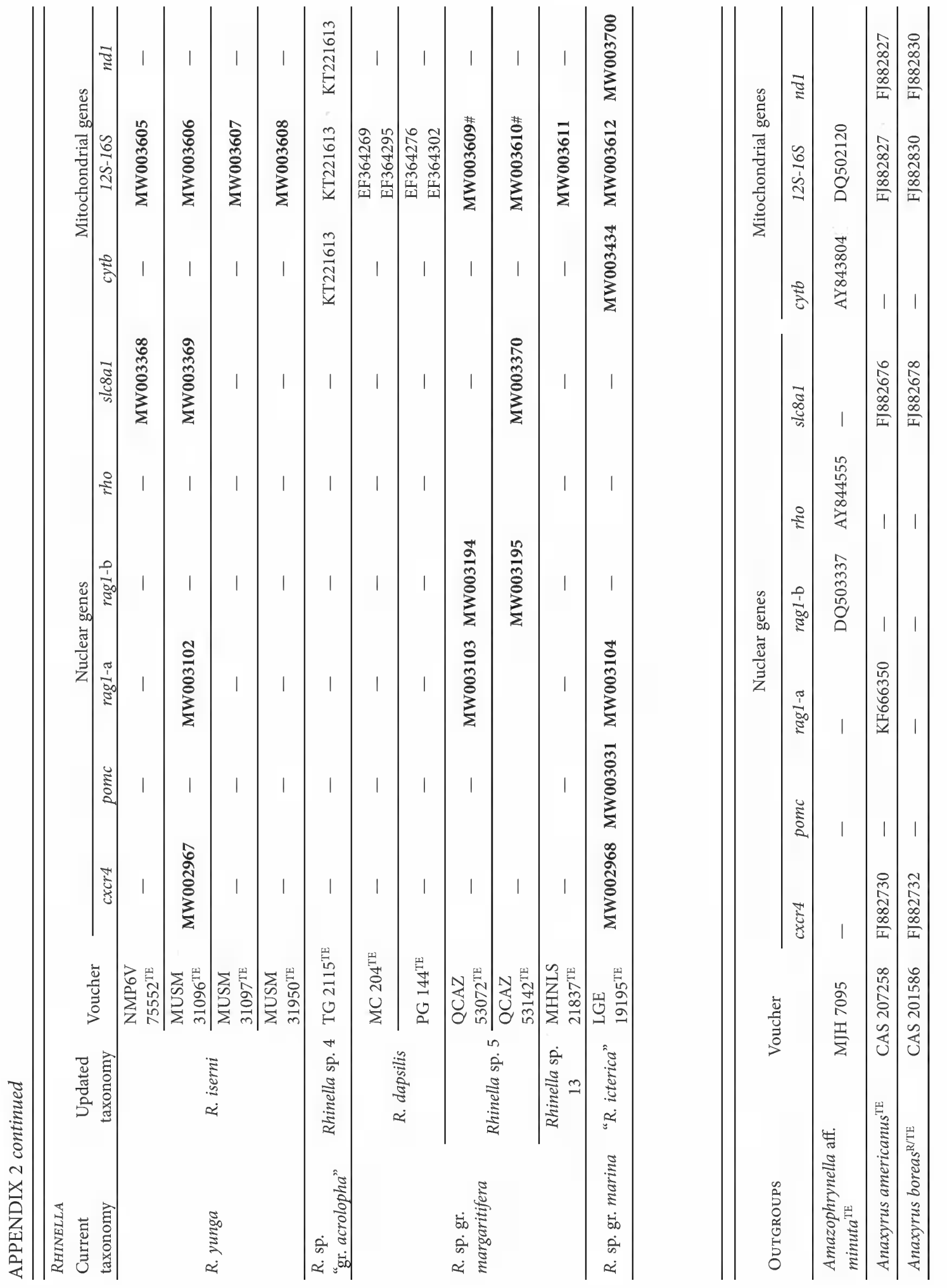




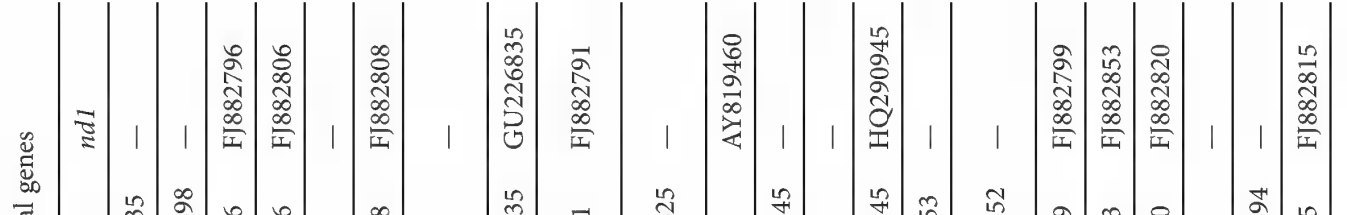

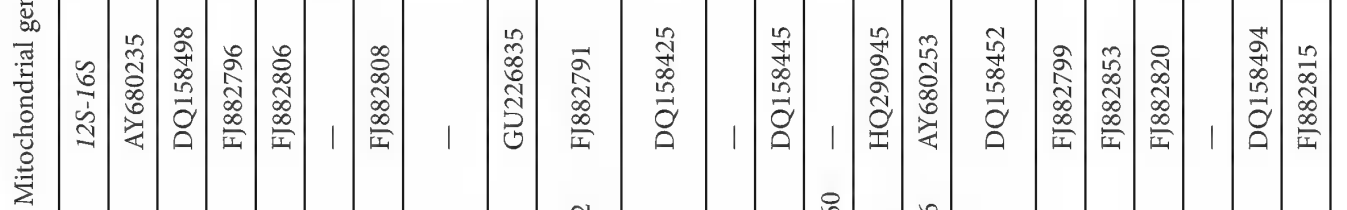
ํํㄴ

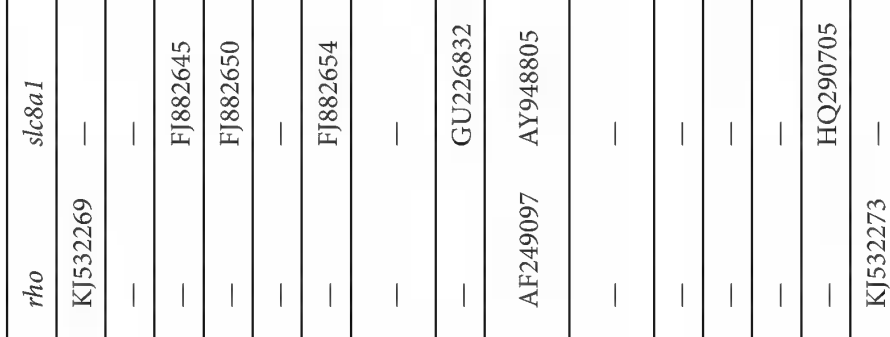

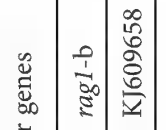

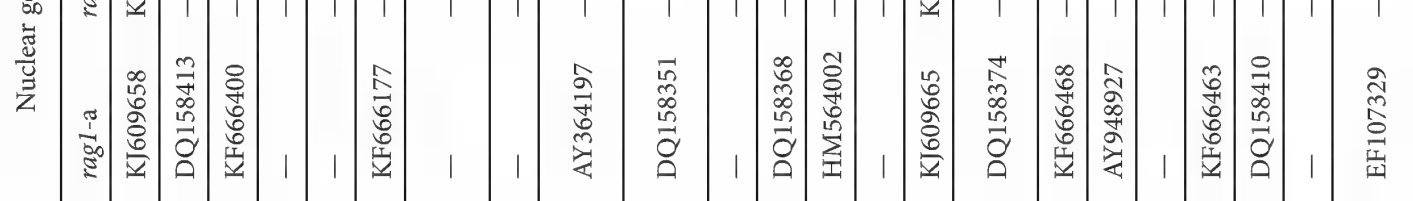

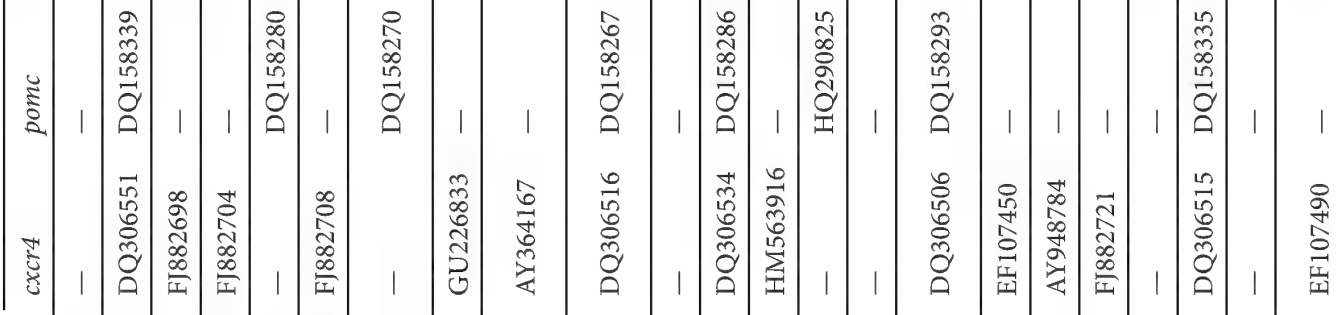

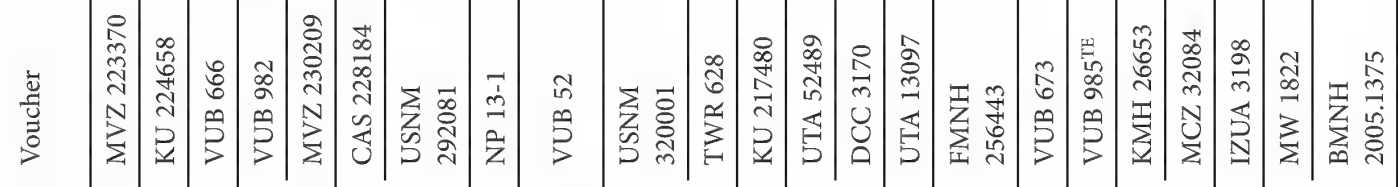

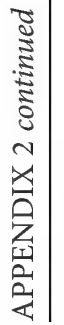

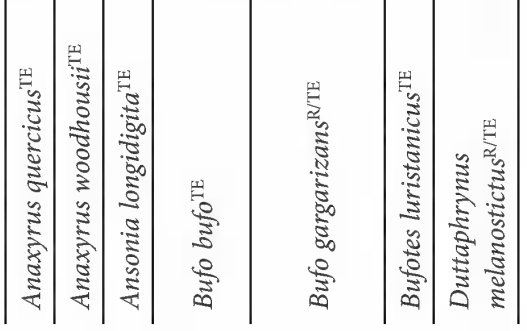

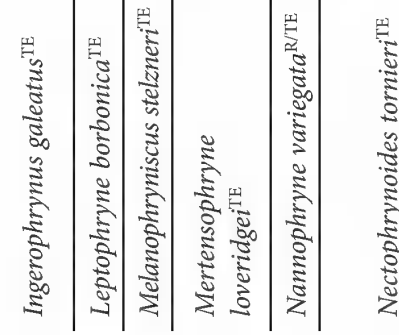




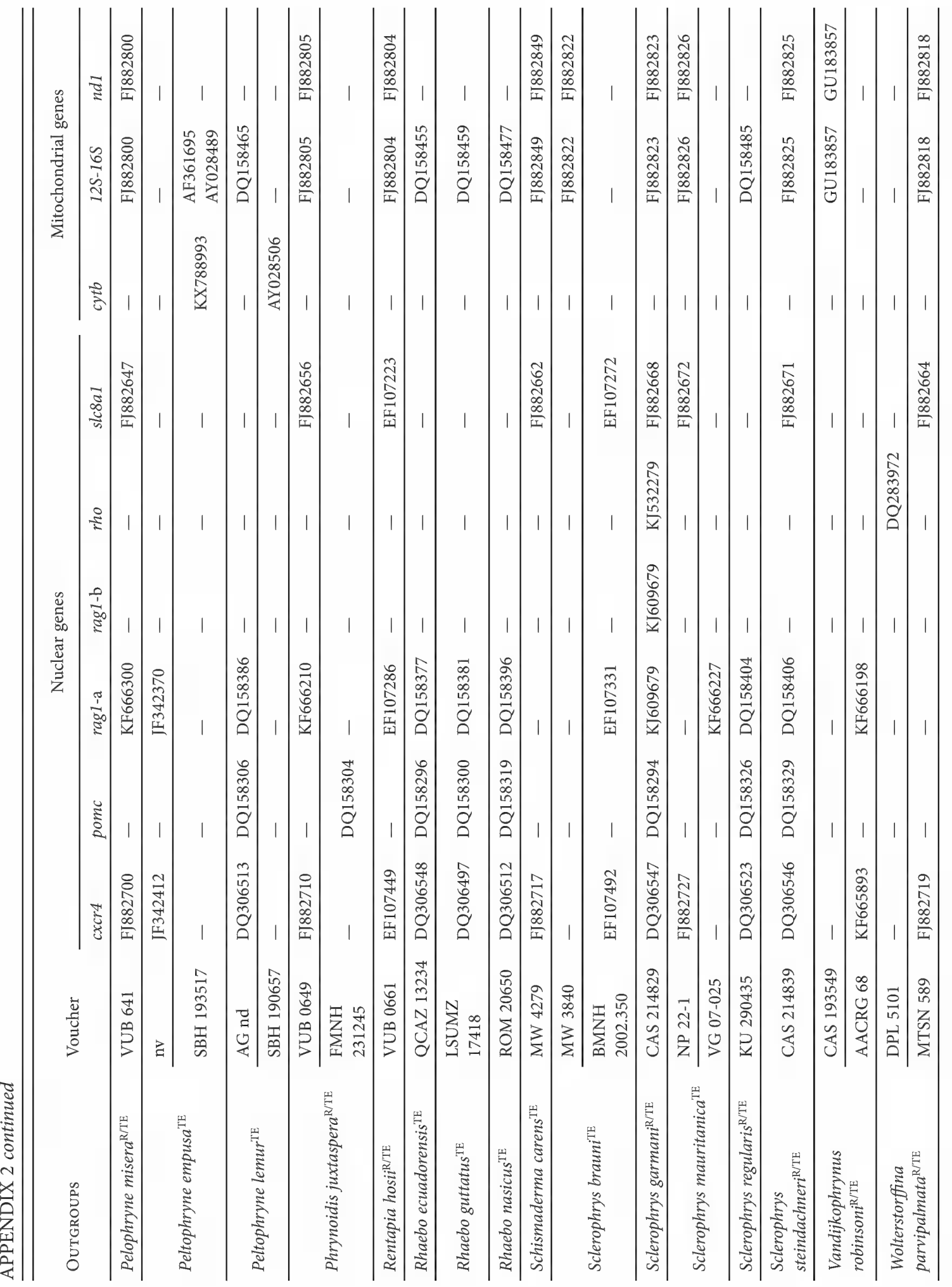




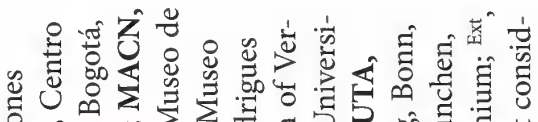

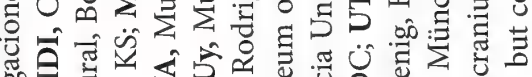

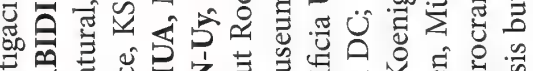

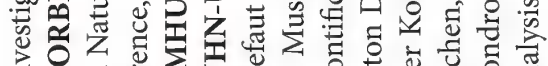

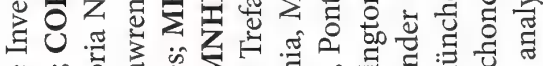

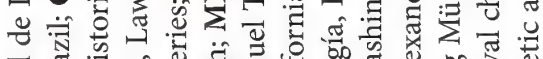

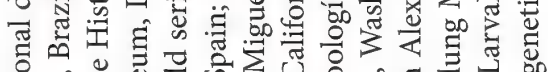

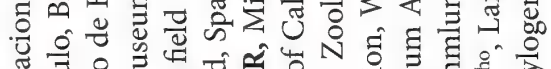

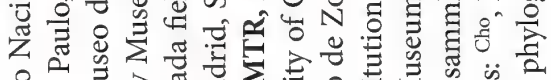

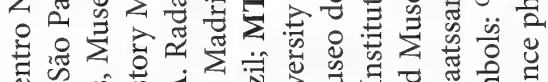
Uूँ

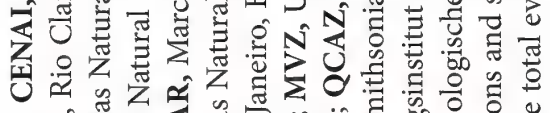

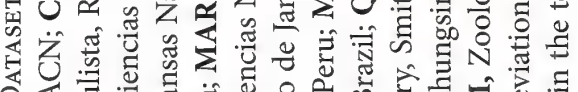

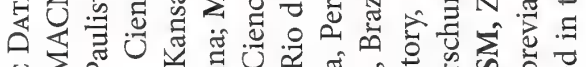

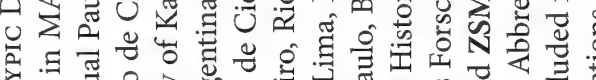

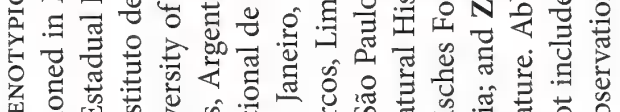

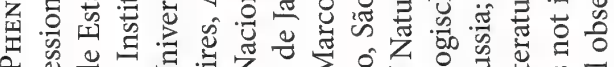
क 凶 ค

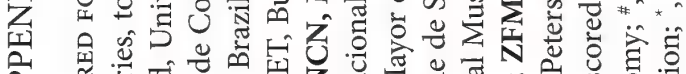

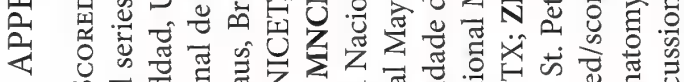

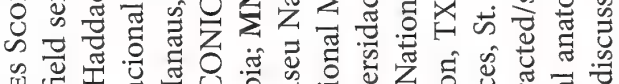

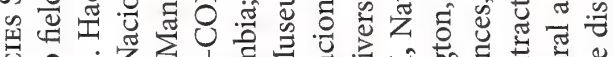
일 के

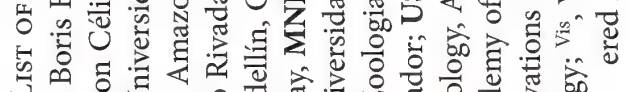
นิ थै

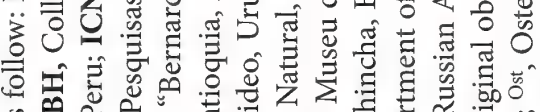

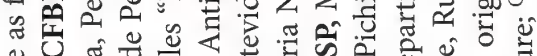

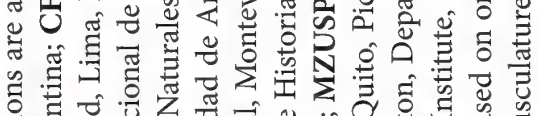

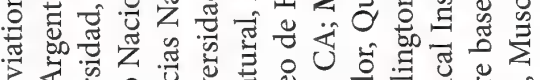

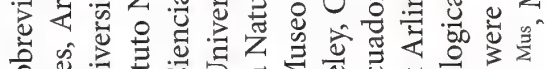
की

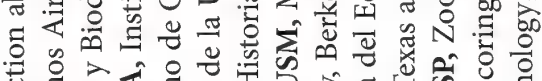

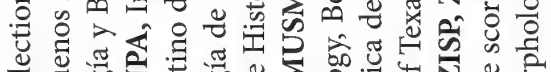

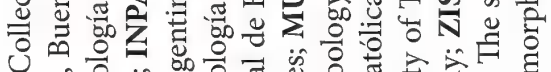

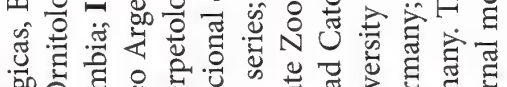

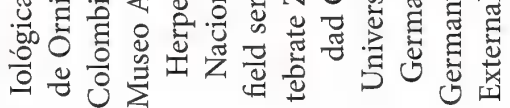

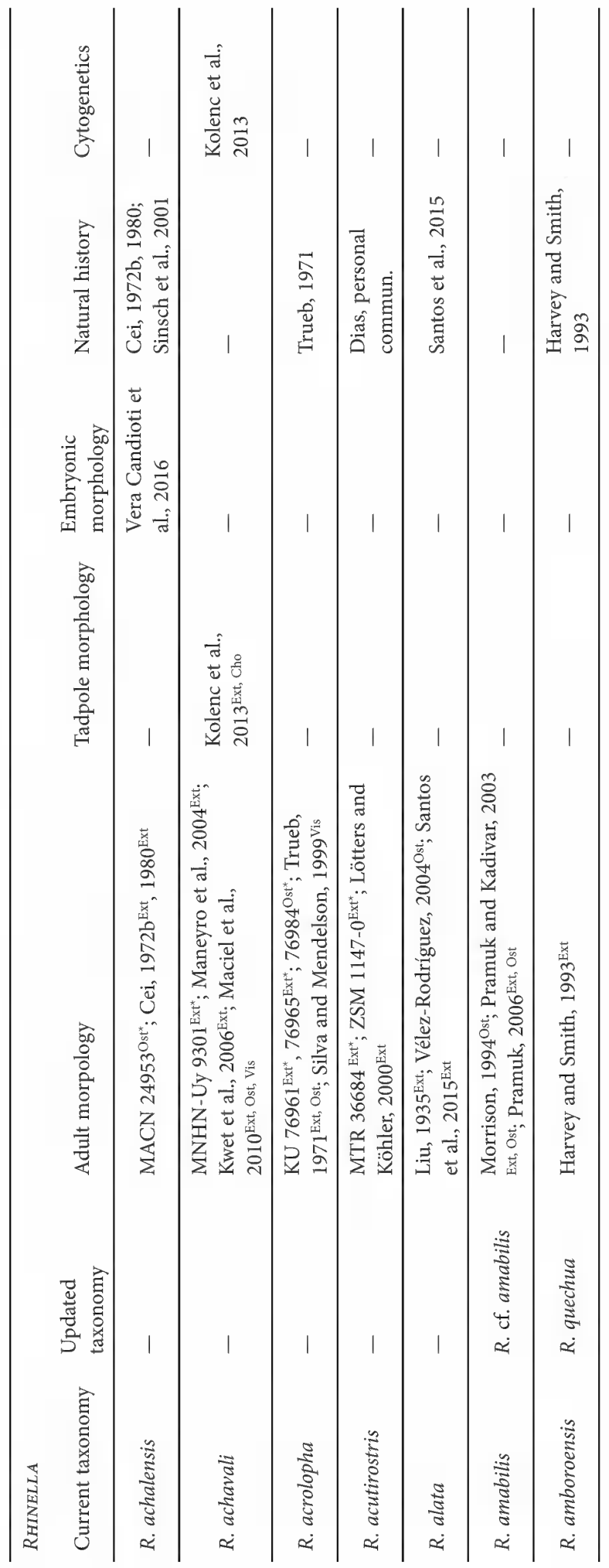




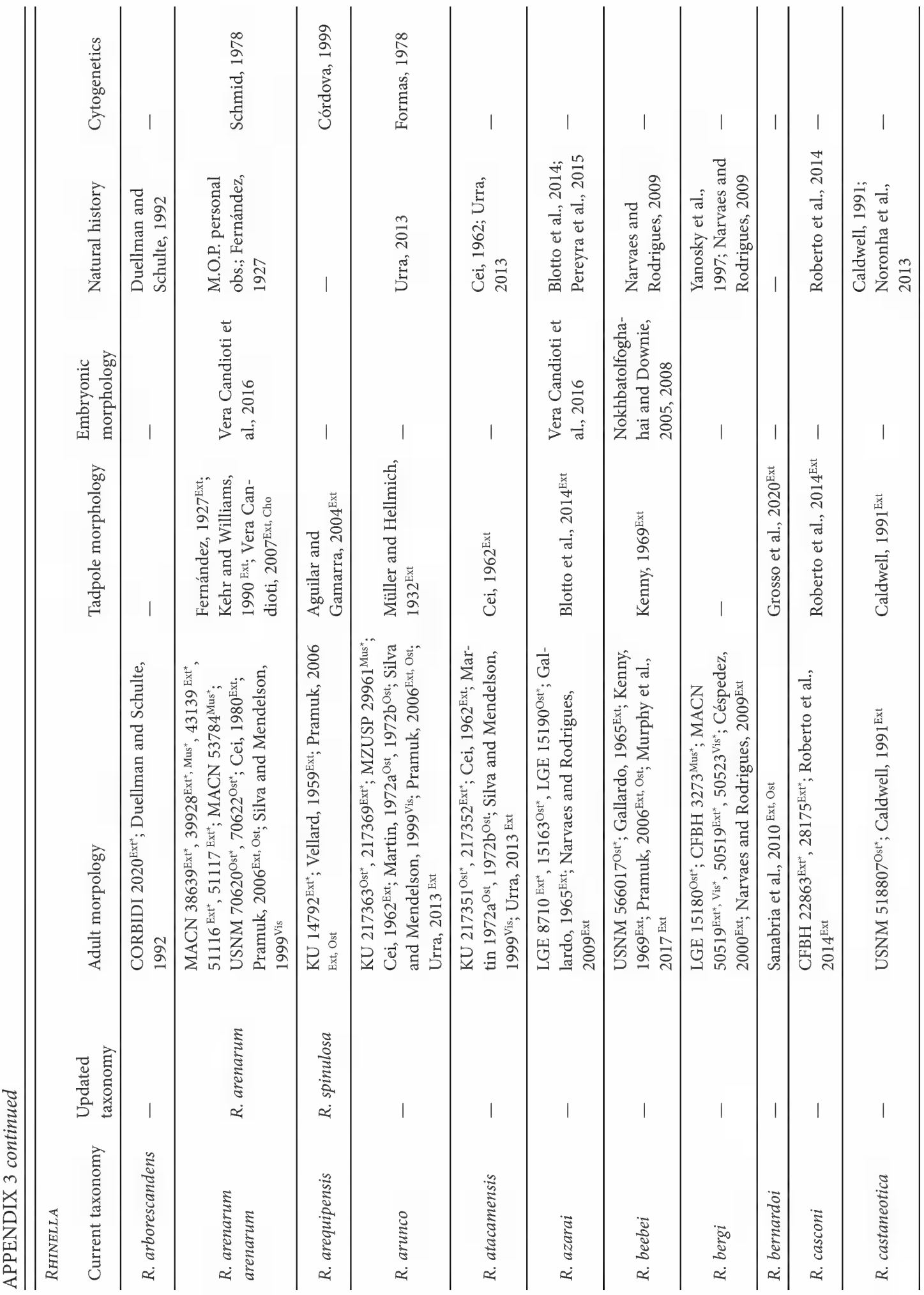




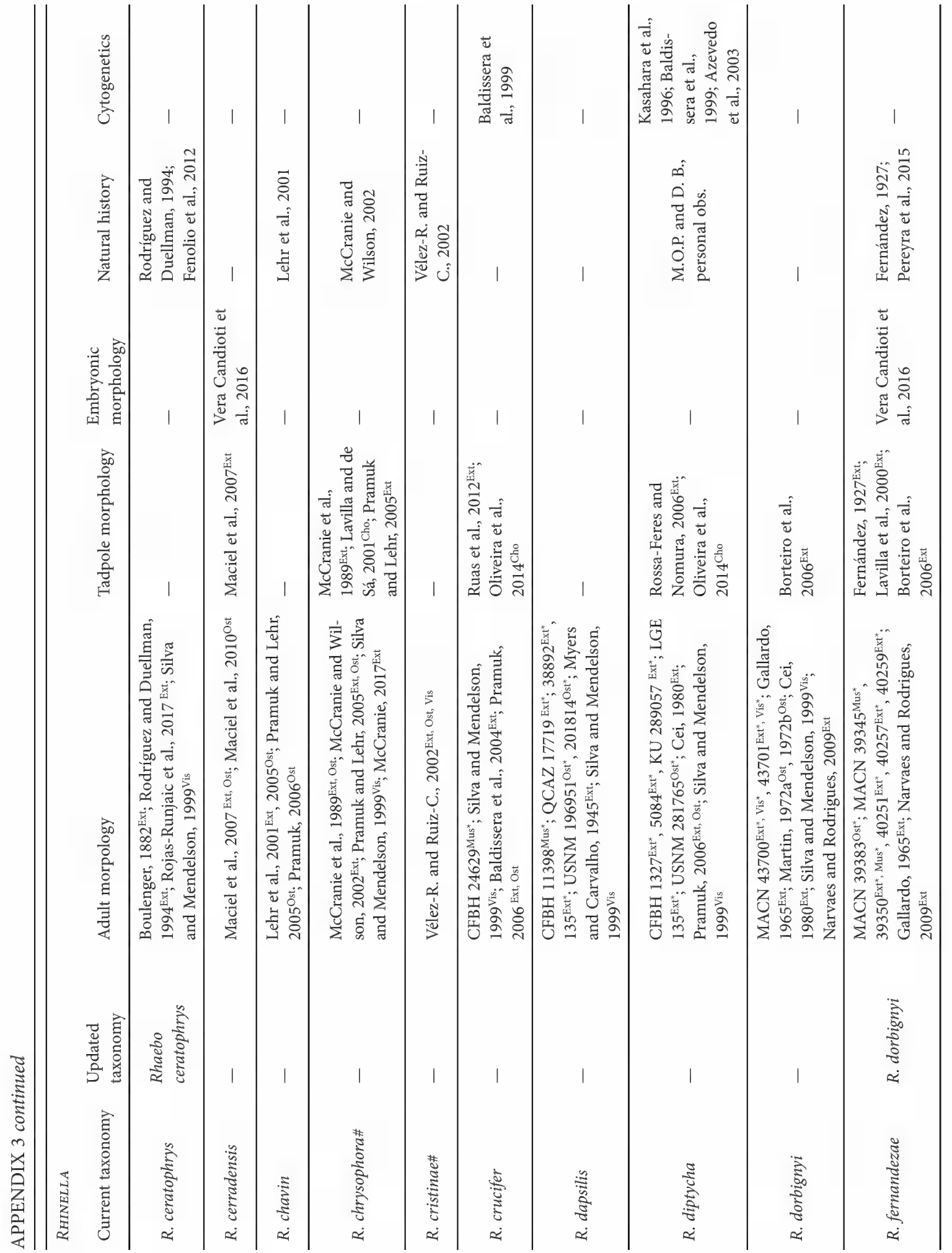




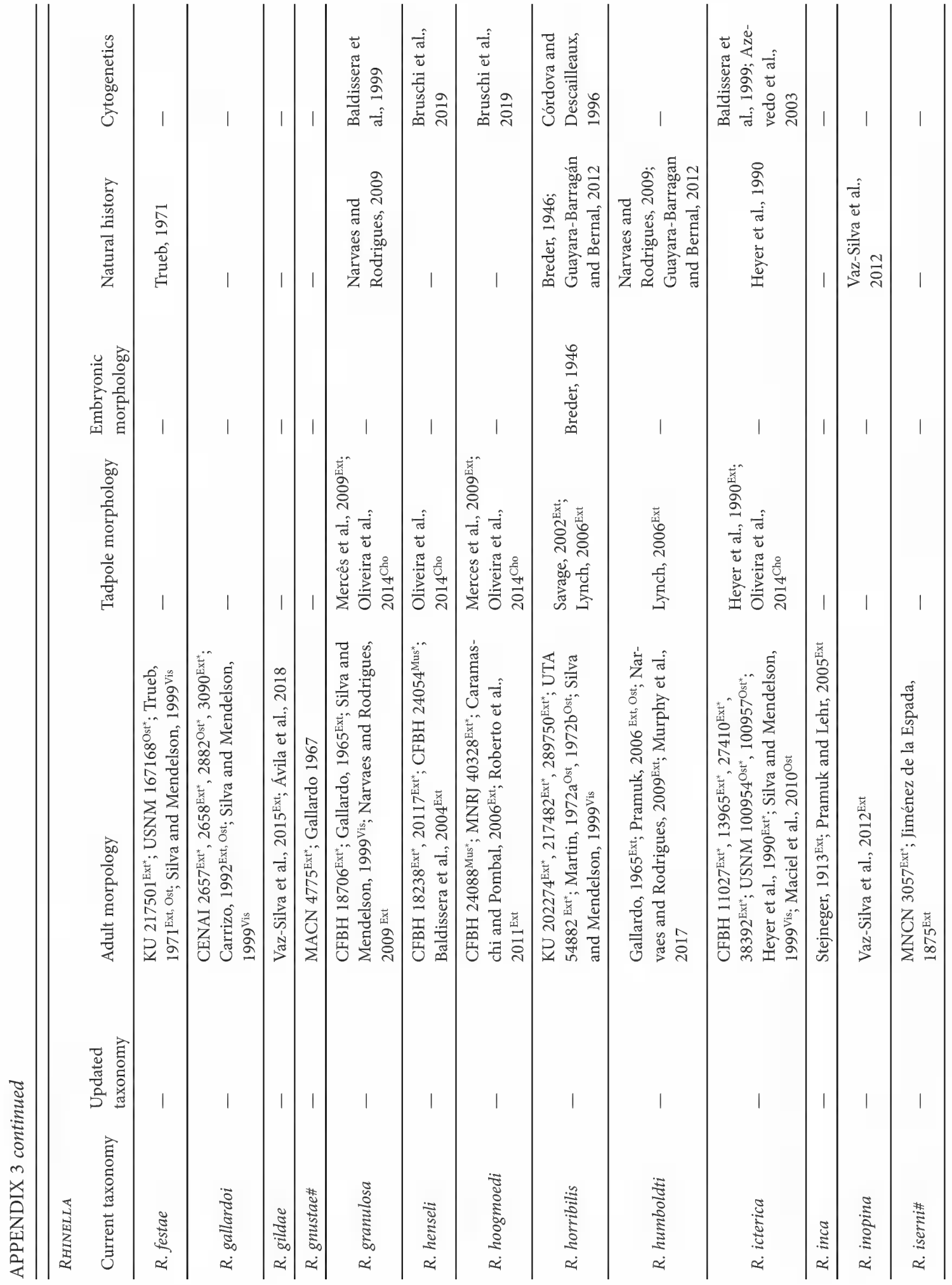




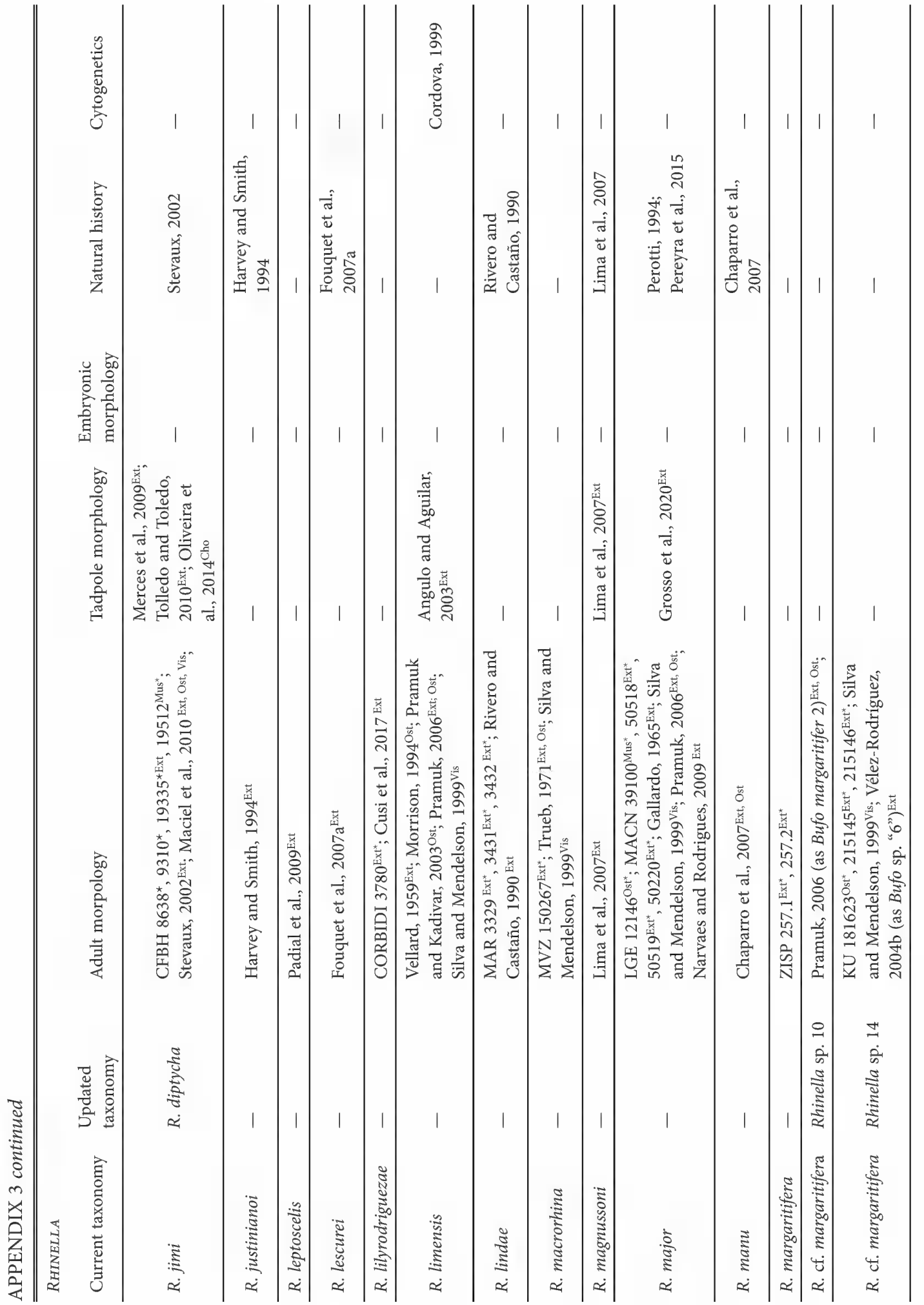




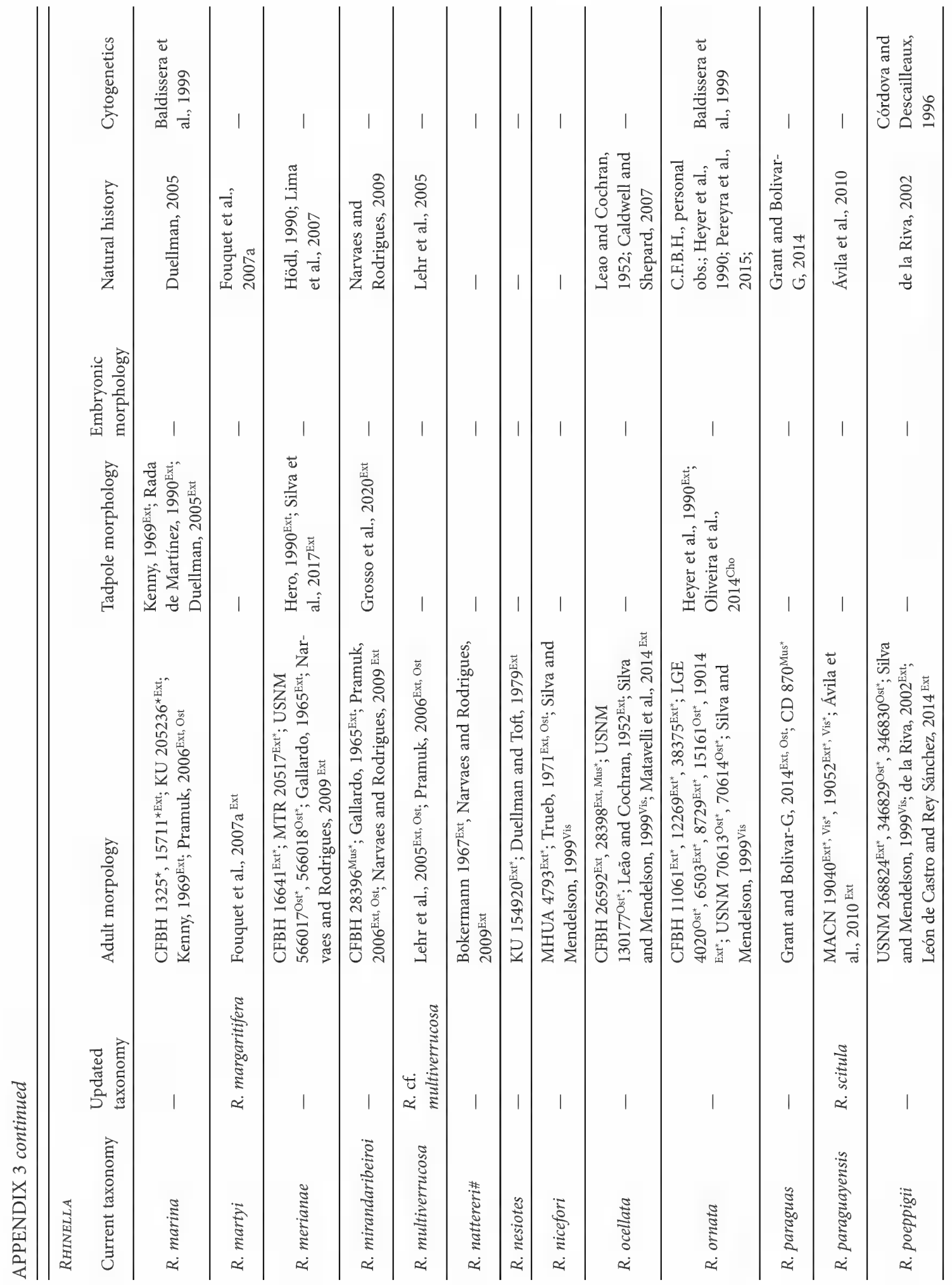




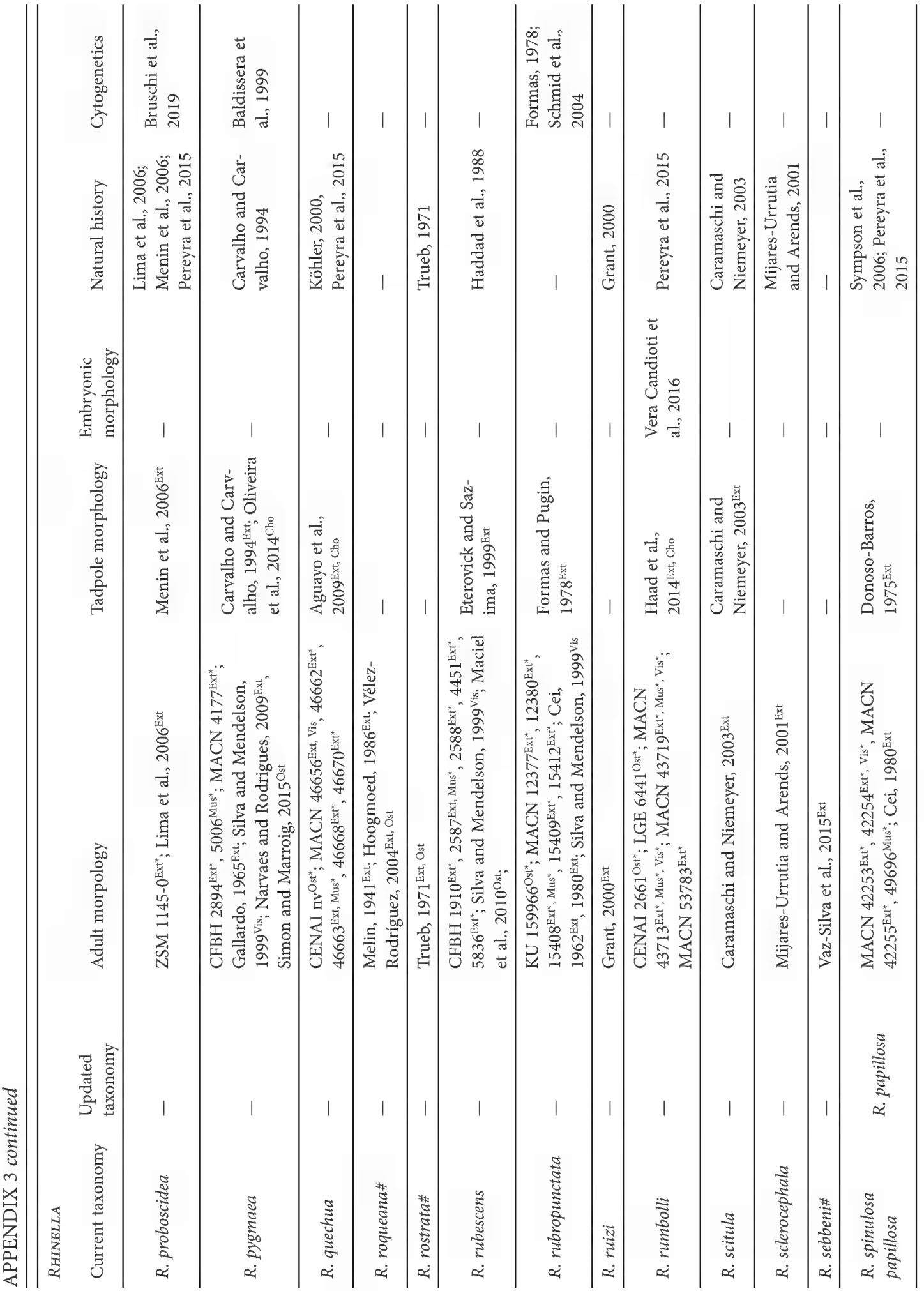




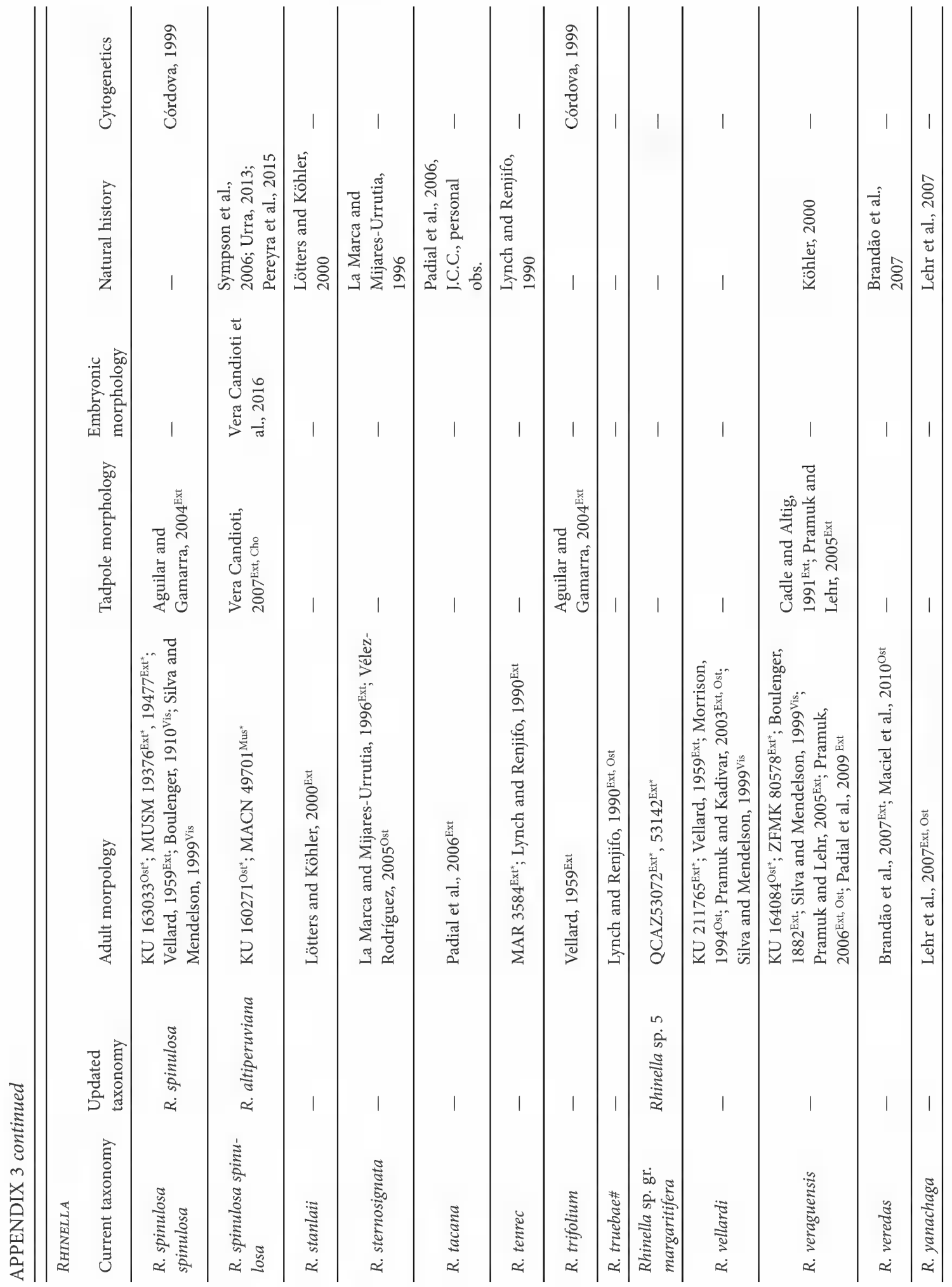




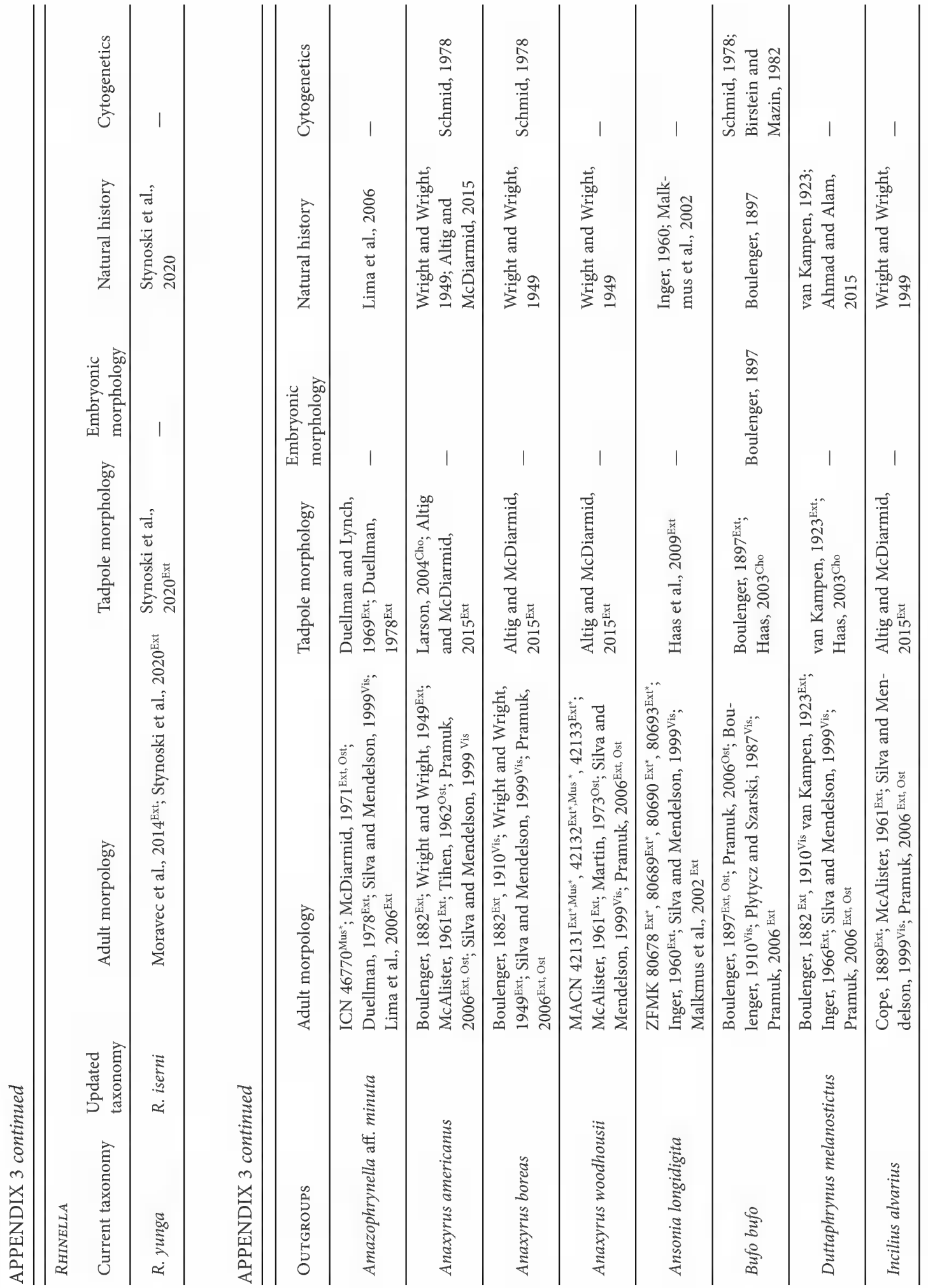




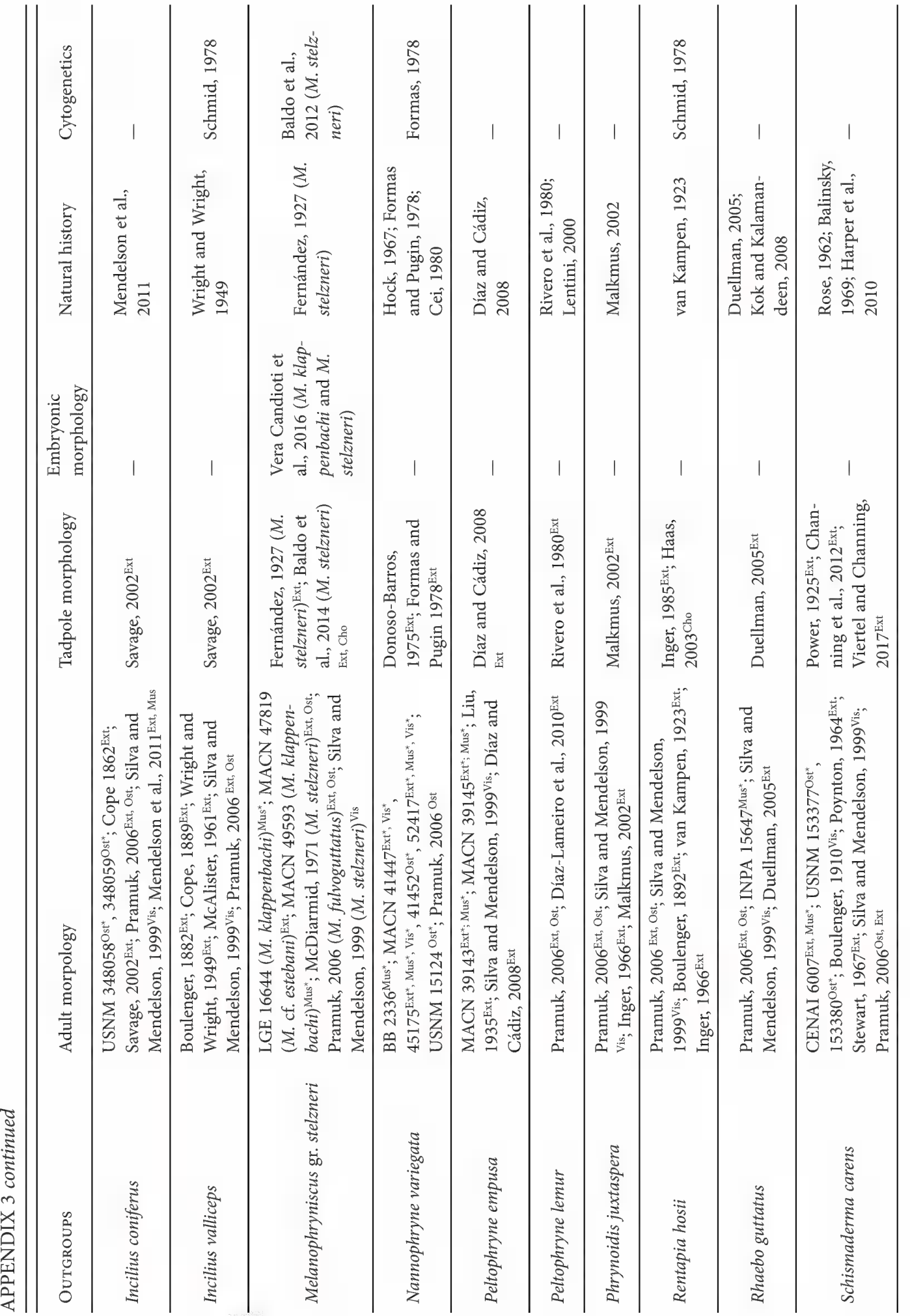




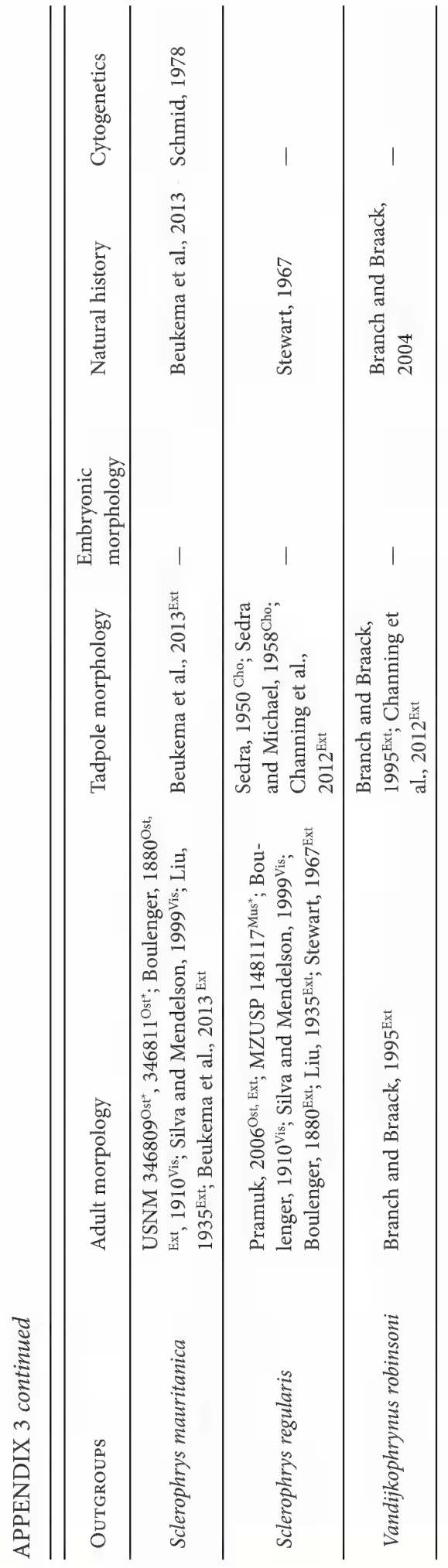




\section{APPENDIX 4}

List of Studied Specimens for the Phenotypic Dataset

Museum specimens used to score the character states reported in appendix 3. Species are listed following the taxonomic changes implemented in this study (synonyms between quotation marks).

See appendix 3 for institutional codes details. Abbreviations: C\&S, cleared and stained specimen; $\mu \mathrm{CT}$, Tridimensional osteological reconstructions of the specimen; DSk, dry skeleton; F, female;

M, male; SA, subadult; nd, not determined.

\begin{tabular}{|c|c|c|c|c|c|}
\hline \multicolumn{6}{|l|}{ RHINELLA } \\
\hline Current taxonomy & $\begin{array}{l}\text { Updated } \\
\text { taxonomy }\end{array}$ & Acronym & Locality & Sex & Observations \\
\hline R. achalensis & - & MACN 24953 & $\begin{array}{l}\text { Argentina: Córdoba: San Alberto, Pampa de } \\
\text { Achala }\end{array}$ & nd & $\mathrm{C} \& \mathrm{~S}$ \\
\hline R. achavali & - & $\begin{array}{l}\text { MNHN-Uy } \\
9301\end{array}$ & $\begin{array}{l}\text { Uruguay: Treinta y Tres: Quebrada de los } \\
\text { Cuervos }\end{array}$ & nd & - \\
\hline \multirow{3}{*}{ R. acrolopha } & \multirow{3}{*}{-} & KU 76961 & Panama: Darién: Cerro Mali & SA & - \\
\hline & & KU 76965 & Panama: Darién: Cerro Mali & M & - \\
\hline & & KU 76984 & Colombia: Chocó: $\mathrm{N}$ slope Cerro Mali & SA & C\&S \\
\hline \multirow[t]{2}{*}{ R. acutirostris } & \multirow[t]{2}{*}{-} & MTR 36684 & $\begin{array}{l}\text { Brazil: Amazonas: Comunidade Indígena } \\
\text { Caiuá, Rio Içá }\end{array}$ & M & - \\
\hline & & ZSM 1147-0 & Brazil: "flumen Amazonum" & M & holotype \\
\hline R. arborescandens & - & CORBIDI 2020 & Peru: Amazonas: Bagua & nd & - \\
\hline \multirow{8}{*}{$\begin{array}{l}R . \text { arenarum } \\
\text { arenarum }\end{array}$} & \multirow{8}{*}{ R. arenarum } & MACN 38639 & Argentina: San Luis: Ayacucho & $\mathrm{F}$ & - \\
\hline & & MACN 39928 & Argentina: Ciudad de Buenos Aires & $\mathrm{F}$ & - \\
\hline & & MACN 43139 & $\begin{array}{l}\text { Argentina: San Luis: Junín, Santa Rosa de } \\
\text { Conlara }\end{array}$ & M & - \\
\hline & & MACN 51116 & $\begin{array}{l}\text { Argentina: San Luis: Pringles, near La Caro- } \\
\text { lina }\end{array}$ & nd & - \\
\hline & & MACN 51117 & $\begin{array}{l}\text { Argentina: San Luis: Pringles, near La Caro- } \\
\text { lina }\end{array}$ & nd & - \\
\hline & & MACN 53784 & $\begin{array}{l}\text { Argentina: Salta: Santa Victoria, Quebrada } \\
\text { "El Lapachar" }\end{array}$ & M & - \\
\hline & & USNM 70620 & Uruguay: Montevideo: Montevideo & nd & $\begin{array}{l}\mu \mathrm{CT} \text { (Morpho- } \\
\text { source ID 22592) }\end{array}$ \\
\hline & & USNM 70622 & Uruguay: Montevideo: Montevideo & nd & $\begin{array}{l}\mu \mathrm{CT} \text { (Morpho- } \\
\text { source ID 22593) }\end{array}$ \\
\hline R. arequipensis & R. spinulosa & KU 14792 & Peru: Arequipa: Zamacola, Cerro Colorado & $\mathrm{F}$ & - \\
\hline \multirow{3}{*}{ R. arunco } & \multirow{3}{*}{-} & KU 217363 & Chile: Santiago: $2 \mathrm{~km}$ S Rungue & SA & $C \& S$ \\
\hline & & KU 217369 & Chile: Santiago: $2 \mathrm{~km}$ S Rungue & SA & - \\
\hline & & MZUSP 29961 & Chile: Santiago: Santiago & $\mathrm{F}$ & - \\
\hline \multirow{2}{*}{ R. atacamensis } & \multirow{2}{*}{-} & KU 217351 & $\begin{array}{l}\text { Chile: Coquimbo: Cuesta Pajonales, } 117 \text { km } \\
\text { N La Serena }\end{array}$ & nd & $\mathrm{C} \& \mathrm{~S}$ \\
\hline & & KU 217352 & $\begin{array}{l}\text { Chile: Coquimbo: Cuesta Pajonales, } 117 \text { km } \\
\text { N La Serena }\end{array}$ & SA & - \\
\hline
\end{tabular}


APPENDIX 4 continued

\begin{tabular}{|c|c|c|c|c|c|}
\hline RHINELLA & & & & & \\
\hline Current taxonomy & $\begin{array}{l}\text { Updated } \\
\text { taxonomy }\end{array}$ & Acronym & Locality & Sex & Observations \\
\hline \multirow{3}{*}{ R. azarai } & \multirow{3}{*}{-} & LGE 15163 & $\begin{array}{l}\text { Argentina: Misiones: Capital: Villa Lanús, } \\
\text { Campus Universidad Nacional de Misiones }\end{array}$ & $\mathrm{F}$ & C\&S \\
\hline & & LGE 15190 & $\begin{array}{l}\text { Argentina: Misiones: Capital: Villa Lanús, } \\
\text { Campus Universidad Nacional de Misiones }\end{array}$ & nd & C\&S \\
\hline & & LGE 8710 & Argentina: Misiones: Candelaria & nd & - \\
\hline R. beebei & - & USNM 566017 & $\begin{array}{l}\text { Guyana: East Berbice: Dubulay Ranch on } \\
\text { the Berbice River }\end{array}$ & nd & $C \& S$ \\
\hline \multirow{2}{*}{ R. bergi } & \multirow{2}{*}{-} & LGE 15180 & $\begin{array}{l}\text { Argentina: Santa Fe: General Obligado, } \\
\text { Ruta } 32,13 \mathrm{~km} \text { S Villa Ana }\end{array}$ & M & - \\
\hline & & CFBH 3273 & $\begin{array}{l}\text { Brazil: Mato Grosso do Sul: Corumbá, } \\
\text { Passo da Lontra }\end{array}$ & M & - \\
\hline \multirow{2}{*}{ R. casconi } & \multirow{2}{*}{ - } & CFBH 22863 & Brazil: Ceará: Guaramiranga & $\mathrm{F}$ & \\
\hline & & CFBH 28175 & Brazil: Ceará: Guaramiranga & M & holotype \\
\hline R. castaneotica & - & USNM 518807 & $\begin{array}{l}\text { Brazil: Pará: Itaituba: Río Tapajos ca. } 65 \text { km } \\
\text { SW of Parque Nacional da Amazônia }\end{array}$ & nd & C\&S \\
\hline \multirow{2}{*}{ R. cerradensis } & \multirow{2}{*}{-} & CHUNB 38670 & Brazil: Bahia: Cocos & M & - \\
\hline & & CHUNB 38671 & Brazil: Bahia: Cocos & M & - \\
\hline R. crucifer & - & CFBH 24629 & Brazil: Bahia: Camacan, Serra Bonita & M & - \\
\hline \multirow{6}{*}{ R. dapsilis } & \multirow{6}{*}{-} & CFBH 11398 & $\begin{array}{l}\text { Tocantins: Babaçulândia: Eixo Ferrovia } \\
\text { Norte-Sul - Brejinho }\end{array}$ & $\mathrm{F}$ & - \\
\hline & & QCAZ 17719 & Ecuador: Napo: Cando & M & - \\
\hline & & QCAZ 38892 & Comunidad Kurintza: Campo Villano & $\mathrm{F}$ & - \\
\hline & & QCAZ 43967 & $\begin{array}{l}\text { Ecuador: Orellana: Parque Nacional Yasuní, } \\
\text { Comunidad Añangu, Río Napo }\end{array}$ & nd & - \\
\hline & & USNM 196951 & $\begin{array}{l}\text { Ecuador: Pastaza: Río Rutuno, tributario } \\
\text { del Río Bobonaza }\end{array}$ & nd & C\&S \\
\hline & & USNM 201814 & Brazil: Amazonas: Borba, Rio Madeira & nd & $\begin{array}{l}\mu \mathrm{CT} \text { (Morpho- } \\
\text { source ID 23326) }\end{array}$ \\
\hline \multirow{5}{*}{ R. diptycha } & \multirow{5}{*}{-} & CFBH 1327 & Brazil: São Paulo: Rio Claro & M & - \\
\hline & & CFBH 5084 & Brazil: São Paulo: Rio Claro & M & - \\
\hline & & KU 289057 & $\begin{array}{l}\text { Paraguay: Concepción: Parque Nacional } \\
\text { Serranía San Luis }\end{array}$ & $\mathrm{F}$ & - \\
\hline & & LGE 135 & $\begin{array}{l}\text { Argentina: Santiago del Estero: } \\
\text { Ojo de Agua }\end{array}$ & M & - \\
\hline & & USNM 281765 & Bolivia: Santa Cruz: Santa Cruz & nd & $\begin{array}{l}\mu \mathrm{CT} \text { (Morpho- } \\
\text { source ID 23334) }\end{array}$ \\
\hline \multirow{2}{*}{ R. dorbignyi } & \multirow{2}{*}{-} & MACN 43700 & Argentina: Buenos Aires: Dolores & $\mathrm{M}$ & - \\
\hline & & MACN 43701 & Argentina: Buenos Aires: Dolores & M & - \\
\hline
\end{tabular}


APPENDIX 4 continued

\begin{tabular}{|c|c|c|c|c|c|}
\hline \multicolumn{6}{|l|}{ RHINELLA } \\
\hline Current taxonomy & $\begin{array}{l}\text { Updated } \\
\text { taxonomy }\end{array}$ & Acronym & Locality & Sex & Observations \\
\hline \multirow{6}{*}{ R. fernandezae } & \multirow{6}{*}{ R. dorbignyi } & MACN 40251 & $\begin{array}{l}\text { Argentina: Chaco: Resistencia, Peaje Gen- } \\
\text { eral Belgrano, Antequera }\end{array}$ & M & - \\
\hline & & MACN 40257 & $\begin{array}{l}\text { Argentina: Chaco: Resistencia, Peaje Gen- } \\
\text { eral Belgrano, Antequera }\end{array}$ & M & - \\
\hline & & MACN 40259 & $\begin{array}{l}\text { Argentina: Chaco: Resistencia, Peaje Gen- } \\
\text { eral Belgrano, Antequera }\end{array}$ & $\mathrm{F}$ & - \\
\hline & & MACN 39345 & Entre Ríos: Islas del Ibicuy & M & - \\
\hline & & MACN 39350 & Entre Ríos: Islas del Ibicuy & nd & - \\
\hline & & MACN 39383 & Entre Ríos: Islas del Ibicuy & $\mathrm{F}$ & $\mathrm{C} \& \mathrm{~S}$ \\
\hline \multirow{2}{*}{ R. festae } & \multirow{2}{*}{-} & USNM 167168 & Ecuador: Pastaza: Alto Río Pucayacu & nd & $\mathrm{C} \& \mathrm{~S}$ \\
\hline & & KU 217501 & Locación Petrolera Garza 1, NE Montalvo & $\mathrm{F}$ & - \\
\hline \multirow{4}{*}{ R. gallardoi } & \multirow{4}{*}{-} & CENAI 2657 & Argentina: Jujuy: Calilegua, Monolito & M & holotype \\
\hline & & CENAI 2658 & Argentina: Jujuy: Calilegua, Monolito & $\mathrm{F}$ & - \\
\hline & & CENAI 2882 & Argentina: Jujuy: Calilegua, Monolito & nd & DSk \\
\hline & & CENAI 3090 & Argentina: Jujuy: Calilegua, Monolito & $\mathrm{F}$ & - \\
\hline R. gnustae\# & - & MACN 4775 & Argentina: Jujuy: Rio Grande & SA & holotype \\
\hline R. granulosa & - & CFBH 18706 & Brazil: Espírito Santo: Linhares & nd & - \\
\hline \multirow{3}{*}{ R. henseli } & \multirow{3}{*}{-} & CFBH 18238 & Brazil: Rio Grande do Sul: Bento Gonçalves & nd & - \\
\hline & & CFBH 20117 & Brazil: Rio Grande do Sul: Catiporã & nd & - \\
\hline & & CFBH 24054 & Brazil: Paraná: São Mateus do Sul & M & - \\
\hline \multirow{2}{*}{ R. hoogmoedi } & \multirow{2}{*}{-} & CFBH 24088 & Brazil: São Paulo: Peruíbe, Trilha 11 & M & - \\
\hline & & MNRJ 40328 & Brazil: Bahia: Canavieiras & $\mathrm{F}$ & paratype \\
\hline \multirow{4}{*}{ R. horribilis } & \multirow[t]{2}{*}{-} & KU 289750 & $\begin{array}{l}\text { El Salvador: Ahuachapan: Parque Nacional } \\
\text { El Imposible, La Fincona }\end{array}$ & $\mathrm{F}$ & - \\
\hline & & UTA 54882 & Mexico: Veracruz: Veracruz & $\mathrm{F}$ & - \\
\hline & \multirow[t]{2}{*}{ Rhinella sp. 1} & KU 202274 & $\begin{array}{l}\text { Ecuador: Pichincha: Tinalandia, } 15.5 \mathrm{~km} \mathrm{SE} \\
\text { Santo Domingo de los Colorados }\end{array}$ & M & - \\
\hline & & KU 217482 & Ecuador: Loja: Vilcabamba & M & - \\
\hline \multirow{6}{*}{ R. icterica } & \multirow{6}{*}{-} & CFBH 11027 & Brazil: Santa Catarina: Bom Jardim da Serra & M & - \\
\hline & & CFBH 13965 & Brazil: Rio de Janeiro: Petrópolis & M & - \\
\hline & & CFBH 27410 & $\begin{array}{l}\text { Brazil: Rio de Janeiro: Município de } \\
\text { Cachoeiras de Macacu, Parque Estadual dos } \\
\text { Três Picos }\end{array}$ & M & - \\
\hline & & CFBH 38392 & Brazil: Rio de Janeiro & $\mathrm{F}$ & - \\
\hline & & USNM 100954 & $\begin{array}{l}\text { Brazil: São Paulo: São Paulo, Parque } \\
\text { Jabaquara }\end{array}$ & nd & $\begin{array}{l}\mu \mathrm{CT} \text { (Morpho- } \\
\text { source ID 23329) }\end{array}$ \\
\hline & & USNM 100957 & $\begin{array}{l}\text { Brazil: São Paulo: São Paulo: Parque } \\
\text { Jabaquara }\end{array}$ & nd & $\begin{array}{l}\mu \mathrm{CT} \text { (Morpho- } \\
\text { source ID 23330) }\end{array}$ \\
\hline R. iserni & - & MNCN 3057 & $\begin{array}{l}\text { Peru: Junin: N.E. Tarma, Andes de Chan- } \\
\text { chamayo }\end{array}$ & $\mathrm{F}$ & holotype \\
\hline
\end{tabular}


APPENDIX 4 continued

\begin{tabular}{|c|c|c|c|c|c|}
\hline \multicolumn{6}{|l|}{ RHINELLA } \\
\hline Current taxonomy & $\begin{array}{l}\text { Updated } \\
\text { taxonomy }\end{array}$ & Acronym & Locality & Sex & Observations \\
\hline \multirow{4}{*}{ R. jimi } & \multirow{4}{*}{ R. diptycha } & CFBH 8638 & Brazil: Pernambuco: Fernando de Noronha & M & - \\
\hline & & CFBH 9310 & Brazil: Pernambuco: Fernando de Noronha & M & - \\
\hline & & CFBH 19335 & Brazil: Bahia, Maracás: Fazenda Cana Brava & $\mathrm{F}$ & - \\
\hline & & CFBH 19512 & Brazil: Bahia, Maracás: Fazenda Cana Brava & M & - \\
\hline R. lilyrodriguezae & - & CORBIDI 3780 & Peru: Huancabamba & $\mathrm{F}$ & - \\
\hline \multirow{3}{*}{ R. lindae } & \multirow{3}{*}{-} & MAR 3329 & $\begin{array}{l}\text { Colombia: Antioquia: Parque Nacional } \\
\text { Natural Las Orquídeas }\end{array}$ & nd & - \\
\hline & & MAR 3431 & $\begin{array}{l}\text { Colombia: Antioquia: Parque Nacional } \\
\text { Natural Las Orquídeas }\end{array}$ & $\mathrm{M}$ & - \\
\hline & & MAR 3432 & $\begin{array}{l}\text { Colombia: Antioquia: Parque Nacional } \\
\text { Natural Las Orquídeas }\end{array}$ & nd & - \\
\hline R. macrorhina\# & - & MVZ 150267 & $\begin{array}{l}\text { Colombia: Antioquia: } 0.5 \mathrm{~km} \text { W (by road) } \\
\text { Medellin }\end{array}$ & nd & - \\
\hline \multirow[t]{2}{*}{ R. major } & \multirow[t]{2}{*}{-} & LGE 12146 & $\begin{array}{l}\text { Argentina: Chaco: General Güemes, near } \\
\text { Wichi }\end{array}$ & M & C\&S \\
\hline & & MACN 39100 & Argentina: Salta: near Dragones & M & - \\
\hline \multirow{3}{*}{ R. cf. margaritifera } & \multirow{3}{*}{$\begin{array}{l}\text { Rhinella sp. } \\
14\end{array}$} & KU 181623 & Peru: Amazonas: near Balsapata & nd & C\&S \\
\hline & & KU 215145 & $\begin{array}{l}\text { Peru: Madre de Dios: Cuzco Amazónico, } 15 \\
\text { km E Puerto Maldonado }\end{array}$ & $\mathrm{F}$ & - \\
\hline & & KU 215146 & $\begin{array}{l}\text { Peru: Madre de Dios: Cuzco Amazónico, } 15 \\
\text { km E Puerto Maldonado }\end{array}$ & $\mathrm{F}$ & - \\
\hline \multirow{2}{*}{ R. margaritifera } & \multirow{2}{*}{-} & ZISP257.1 & "Brasilia" & $\mathrm{F}$ & lectotype \\
\hline & & ZISP257.2 & "Brasilia" & $\mathrm{F}$ & paralectotype \\
\hline \multirow{3}{*}{ R. marina } & \multirow{3}{*}{-} & CFBH 1325 & Brazil: Pará: Paraopebas & M & - \\
\hline & & CFBH 15711 & Brazil: Acre: Tarauacá & nd & - \\
\hline & & KU 205236 & $\begin{array}{l}\text { Peru: Madre de Dios: Cuzco Amazónico, } 15 \\
\text { km E Puerto Maldonado }\end{array}$ & M & - \\
\hline \multirow{4}{*}{ R. merianae } & \multirow{4}{*}{-} & CFBH 16641 & Brazil: Amazonas: Manaus, Reserva Duke & nd & - \\
\hline & & MTR 20517 & $\begin{array}{l}\text { Brazil: Roraima: Estação Ecológica de } \\
\text { Maracá }\end{array}$ & nd & - \\
\hline & & USNM 566017 & Guyana: East Berbice: Dubulay Ranch & nd & $\begin{array}{l}\mu \mathrm{CT} \text { (Morpho- } \\
\text { source ID 23327) }\end{array}$ \\
\hline & & USNM 566018 & Guyana: East Berbice: Dubulay Ranch & nd & $\begin{array}{l}\mu \mathrm{CT} \text { (Morpho- } \\
\text { source ID 23328) }\end{array}$ \\
\hline R. mirandaribeiroi & - & CFBH 28396 & Brazil: Tocantins: Porto Nacional & M & - \\
\hline R. nesiotes & - & KU 154920 & Peru: Huanuco: W slope Serrania de Sira & $\mathrm{F}$ & holotype \\
\hline R. nicefori & R. cf. nicefori & MHUA 4793 & Colombia: Antioquía: Belmira & nd & - \\
\hline \multirow{3}{*}{ R. ocellata } & \multirow{3}{*}{-} & CFBH 26592 & Brazil: Maranhão: Barreirinhas & nd & - \\
\hline & & CFBH 28398 & Brazil: Tocantins: Porto Nacional & $\mathrm{F}$ & - \\
\hline & & USNM 130177 & $\begin{array}{l}\text { Brazil: Goiás: Rio Araguaia, between Santa } \\
\text { Leopoldina and Ilha do Bananal }\end{array}$ & nd & $\begin{array}{l}\mu \mathrm{CT} \text { (Morpho- } \\
\text { source ID 23331) }\end{array}$ \\
\hline
\end{tabular}


APPENDIX 4 continued

\begin{tabular}{|c|c|c|c|c|c|}
\hline \multicolumn{6}{|l|}{ RHINELLA } \\
\hline Current taxonomy & $\begin{array}{l}\text { Updated } \\
\text { taxonomy }\end{array}$ & Acronym & Locality & Sex & Observations \\
\hline \multirow{10}{*}{ R. ornata } & \multirow{10}{*}{-} & CFBH 11061 & Brazil: Paraná: Antonina, Trilha do Ferro & nd & - \\
\hline & & CFBH 12269 & Brazil: São Paulo: Caraguatatuba & nd & - \\
\hline & & CFBH 38375 & Brazil: Rio de Janeiro: Visconde de Mauá & nd & - \\
\hline & & LGE 15161 & $\begin{array}{l}\text { Argentina: Misiones: Cainguás, Aristóbulo } \\
\text { del Valle, Arroyo Cuñá Pirú Chico }\end{array}$ & M & $\mathrm{C} \& \mathrm{~S}$ \\
\hline & & LGE 19014 & $\begin{array}{l}\text { Argentina: Misiones: Iguazú, Parque Pro- } \\
\text { vincial Uruguaí }\end{array}$ & M & - \\
\hline & & LGE 4020 & Argentina: Misiones & nd & $\mathrm{C} \& \mathrm{~S}$ \\
\hline & & LGE 6503 & $\begin{array}{l}\text { Argentina: Misiones: Cainguás, Aristóbulo } \\
\text { del Valle, Arroyo Cuñá Pirú Chico }\end{array}$ & & - \\
\hline & & LGE 8729 & $\begin{array}{l}\text { Argentina: Misiones: Capital, Profundidad, } \\
\text { Parque Provincial Profundidad }\end{array}$ & nd & - \\
\hline & & USNM 70613 & Brazil: Rio de Janeiro: Rio de Janeiro & $\mathrm{F}$ & $\begin{array}{l}\mu \mathrm{CT} \text { (Morpho- } \\
\text { source ID 22596) }\end{array}$ \\
\hline & & USNM 70614 & Brazil: Rio de Janeiro: Rio de Janeiro & $\mathrm{F}$ & $\begin{array}{l}\mu \mathrm{CT} \text { (Morpho- } \\
\text { source ID 22597) }\end{array}$ \\
\hline R. paraguas & - & CD 870 & Colombia: Valle del Cauca: Dagua & M & - \\
\hline \multirow{2}{*}{ R. paraguayensis } & \multirow{2}{*}{ R. scitula } & MACN 19040 & $\begin{array}{l}\text { Bolivia: Santa Cruz: Santa Cruz de la Sierra, } \\
\text { Surutú, Río Colorado }\end{array}$ & nd & - \\
\hline & & MACN 19052 & $\begin{array}{l}\text { Bolivia: Santa Cruz: Santa Cruz de la Sierra, } \\
\text { Surutú, Río Colorado }\end{array}$ & nd & - \\
\hline \multirow{2}{*}{ R. poeppigii } & \multirow{2}{*}{-} & USNM 346829 & Peru: San Martín: Aucayacu, Río Huallaga & nd & $\begin{array}{l}\mu \mathrm{CT} \text { (Morpho- } \\
\text { source ID 23332) }\end{array}$ \\
\hline & & USNM 346830 & Peru: San Martín: Aucayacu, Río Huallaga & nd & $\begin{array}{l}\mu \mathrm{CT} \text { (Morpho- } \\
\text { source ID 23333) }\end{array}$ \\
\hline \multirow{3}{*}{ R. pygmaea } & \multirow{3}{*}{-} & CFBH 2894 & Brazil: Bahia: Ilhéus & nd & - \\
\hline & & CFBH 5006 & Brazil: Rio de Janeiro: São João da Barra & M & - \\
\hline & & MACN 4177 & Brazil: Rio de Janeiro: São João da Barra & nd & paratype \\
\hline \multirow{6}{*}{ R. quechua } & \multirow{6}{*}{-} & CENAI ND & Bolivia: Carrasco: Cochabamba & SA & DSk \\
\hline & & MACN 46656 & Bolivia: Carrasco: Cochabamba & $\mathrm{F}$ & - \\
\hline & & MACN 46662 & Bolivia: Carrasco: Cochabamba & M & - \\
\hline & & MACN 46663 & Bolivia: Carrasco: Cochabamba & M & - \\
\hline & & MACN 46668 & Bolivia: Carrasco: Cochabamba & M. & - \\
\hline & & MACN 46670 & Bolivia: Carrasco: Cochabamba & M. & - \\
\hline \multirow{5}{*}{ R. rubescens } & \multirow{5}{*}{-} & CFBH 2587 & Brazil: Distrito Federal: Brasília & M & - \\
\hline & & CFBH 1910 & Brazil: Distrito Federal: Brasília & M & - \\
\hline & & CFBH 2587 & Brazil: Distrito Federal: Brasília & M & - \\
\hline & & CFBH 2588 & Brazil: Distrito Federal: Brasília & M & - \\
\hline & & CFBH 4451 & $\begin{array}{l}\text { Brazil: Minas Gerais: Morro do Ferro, } \\
\text { Poços de Caldas }\end{array}$ & $\mathrm{F}$ & - \\
\hline
\end{tabular}


APPENDIX 4 continued

\begin{tabular}{|c|c|c|c|c|c|}
\hline \multicolumn{6}{|l|}{ RHINELLA } \\
\hline Current taxonomy & $\begin{array}{l}\text { Updated } \\
\text { taxonomy }\end{array}$ & Acronym & Locality & Sex & Observations \\
\hline R. rubescens & - & CFBH 5836 & $\begin{array}{l}\text { Brazil: Minas Gerais: Morro do Ferro, } \\
\text { Poços de Caldas }\end{array}$ & SA & - \\
\hline \multirow{6}{*}{ R. rubropunctata } & \multirow{6}{*}{-} & KU 159966 & Chile, Llanquihue: Lago Todos Los Santos & nd & $\mathrm{C} \& \mathrm{~S}$ \\
\hline & & MACN 15408 & Argentina: Río Negro: Bariloche, El Bolsón & $\mathrm{F}$ & \\
\hline & & MACN 12377 & Argentina: Río Negro: Bariloche, El Bolsón & $\mathrm{F}$ & - \\
\hline & & MACN 12380 & Argentina: Río Negro: Bariloche, El Bolsón & M & - \\
\hline & & MACN 15409 & Argentina: Río Negro: Bariloche, El Bolsón & $\mathrm{F}$ & - \\
\hline & & MACN 15412 & Argentina: Río Negro: Bariloche, El Bolsón & M & - \\
\hline \multirow{5}{*}{ R. rumbolli } & \multirow{5}{*}{-} & CENAI 2661 & Argentina: Jujuy: Calilegua, Monolito & nd & DSk \\
\hline & & LGE 6441 & $\begin{array}{l}\text { Argentina: Jujuy: Doctor Manuel Belgrano, } \\
\text { Ocloyas }\end{array}$ & M & C\&S \\
\hline & & MACN 43713 & $\begin{array}{l}\text { Argentina: Salta: Santa Victoria, Parque } \\
\text { Nacional Baritú }\end{array}$ & M & - \\
\hline & & MACN 43719 & $\begin{array}{l}\text { Argentina: Salta: Santa Victoria, Parque } \\
\text { Nacional Baritú }\end{array}$ & M & - \\
\hline & & MACN 53783 & $\begin{array}{l}\text { Argentina: Salta: Santa Victoria, Parque } \\
\text { Nacional Baritú }\end{array}$ & $\mathrm{F}$ & - \\
\hline \multirow{4}{*}{$\begin{array}{l}\text { R. spinulosa } \\
\text { papillosa }\end{array}$} & \multirow{4}{*}{ R. papillosa } & MACN 49696 & Argentina: Rio Negro: Bariloche & M & - \\
\hline & & MACN 42253 & $\begin{array}{l}\text { Argentina: Río Negro: Bariloche, Pampa } \\
\text { Linda, near Cerro Tronador }\end{array}$ & $\mathrm{F}$ & - \\
\hline & & MACN 42254 & $\begin{array}{l}\text { Argentina: Río Negro: Bariloche, Pampa } \\
\text { Linda, near Cerro Tronador }\end{array}$ & M & - \\
\hline & & MACN 42255 & $\begin{array}{l}\text { Argentina: Río Negro: Bariloche, Pampa } \\
\text { Linda, near Cerro Tronador }\end{array}$ & M & - \\
\hline \multirow{4}{*}{$\begin{array}{l}\text { R. spinulosa } \\
\text { spinulosa }\end{array}$} & \multirow{3}{*}{ R. spinulosa } & KU 163033 & Peru: Puno: 4 km W Santa Rosa & nd & $\mathrm{C} \& \mathrm{~S}$ \\
\hline & & MUSM 19376 & Peru: Puno & $\mathrm{F}$ & - \\
\hline & & MUSM 19477 & Peru: Puno & M & - \\
\hline & $\begin{array}{l}R . \\
\text { altiperuviana }\end{array}$ & MACN 49701 & Argentina: Jujuy: Quebrada de Sepultura & M & - \\
\hline R. tenrec & - & MAR 3584 & $\begin{array}{l}\text { Colombia: Antioquia: Parque Nacional } \\
\text { Natural Las Orquídeas }\end{array}$ & nd & - \\
\hline \multirow{2}{*}{$\begin{array}{l}\text { Rhinella sp. gr. } \\
\text { margaritifera }\end{array}$} & \multirow{2}{*}{ Rhinella sp. 5} & QCAZ 53072 & Ecuador: Pastaza: Montalvo & M & - \\
\hline & & QCAZ 53142 & Ecuador: Pastaza: Montalvo & $\mathrm{F}$ & - \\
\hline R. vellardi & - & KU 211765 & $\begin{array}{l}\text { Peru: Cajamarca: Cajabamba, } 10 \mathrm{~km} \text { SSE } \\
\text { Cajabamba }\end{array}$ & nd & - \\
\hline \multirow{2}{*}{ R. veraguensis } & & ZFMK 80578 & $\begin{array}{l}\text { Bolivia: La Paz: between Caranavi and Palos } \\
\text { Blancos }\end{array}$ & M & - \\
\hline & - & KU 164084 & $\begin{array}{l}\text { Peru: Cusco: } 4 \text { km SW Santa Isabel, Rio } \\
\text { Cosnipata }\end{array}$ & M & DSk \\
\hline
\end{tabular}


APPENDIX 4 continued

\begin{tabular}{|c|c|c|c|c|}
\hline OUTGROUPS & Acronym & Locality & Sex & Observations \\
\hline Amazophrynella aff. minuta & ICN 46770 & Colombia: Amazonas: Leticia & M & - \\
\hline \multirow{3}{*}{ Anaxyrus woodhousii } & MACN 42131 & ND (Pet trade) & $\mathrm{M}$ & - \\
\hline & MACN 42132 & ND (Pet trade) & M & - \\
\hline & MACN 42133 & ND (Pet trade) & $\mathrm{M}$ & - \\
\hline \multirow{4}{*}{ Ansonia longidigita } & ZFMK 80678 & Malaysia: Sabah: Mt. Kinabalu & $\mathrm{M}$ & - \\
\hline & ZFMK 80689 & Malaysia: Sabah: Mt. Kinabalu & M & - \\
\hline & ZFMK 80690 & Malaysia: Sabah: Mt. Kinabalu & M & - \\
\hline & ZFMK 80693 & Malaysia: Sabah: Mt. Kinabalu & M & - \\
\hline \multirow{2}{*}{ Incilius coniferus } & USNM 348058 & $\begin{array}{l}\text { Panama: Bocas del Toro: Isla } \\
\text { Cristobal }\end{array}$ & $\mathrm{F}$ & $\begin{array}{l}\mu \mathrm{CT} \text { (Morphosource ID } \\
21896)\end{array}$ \\
\hline & USNM 348059 & $\begin{array}{l}\text { Panama: Bocas del Toro: Isla } \\
\text { Cristobal }\end{array}$ & $\mathrm{F}$ & $\begin{array}{l}\mu \mathrm{CT} \text { (Morphosource ID } \\
21897 \text { ) }\end{array}$ \\
\hline \multirow{3}{*}{$\begin{array}{l}\text { Melanophryniscus } \\
\text { stelzneri group }\end{array}$} & $\begin{array}{l}\text { LGE } 16644 \\
\text { (M. klappenbachi) }\end{array}$ & $\begin{array}{l}\text { Argentina: Chaco: San Fer- } \\
\text { nando, Club Sixty Resistencia }\end{array}$ & nd & - \\
\hline & $\begin{array}{l}\text { MACN } 47819 \\
\text { (M. cf. estebani) }\end{array}$ & $\begin{array}{l}\text { Argentina: San Luis: Libertador } \\
\text { General San Martín, Dique La } \\
\text { Huertita }\end{array}$ & M & - \\
\hline & $\begin{array}{l}\text { MACN } 49593 \\
\text { (M. klappenbachi) }\end{array}$ & $\begin{array}{l}\text { Argentina: Chaco: San Fer- } \\
\text { nando, Club Sixty Resistencia }\end{array}$ & nd & - \\
\hline \multirow{5}{*}{ Nannophryne variegata } & MACN 52417 & $\begin{array}{l}\text { Argentina: Santa Cruz: Lago } \\
\text { Argentino, Lago del Desierto }\end{array}$ & M & - \\
\hline & BB 2336 & $\begin{array}{l}\text { Argentina: Santa Cruz: Lago } \\
\text { Argentino, Lago del Desierto }\end{array}$ & nd & - \\
\hline & MACN 41452 & $\begin{array}{l}\text { Argentina: Santa Cruz: Lago } \\
\text { Argentino, Lago del Desierto }\end{array}$ & M & $\mathrm{C} \& \mathrm{~S}$ \\
\hline & MACN 41475 & $\begin{array}{l}\text { Argentina: Santa Cruz: Lago } \\
\text { Argentino, Lago del Desierto }\end{array}$ & nd & - \\
\hline & USNM 15124 & $\begin{array}{l}\text { Chile: Magallanes: Mayne Har- } \\
\text { bor }\end{array}$ & nd & $\begin{array}{l}\mu \mathrm{CT} \text { (Morphosource ID } \\
21909 \text { ) }\end{array}$ \\
\hline \multirow{2}{*}{ Peltophryne empusa } & MACN 39143 & $\begin{array}{l}\text { Cuba: Isla de la Juventud: } 14.7 \\
\text { mi al SSW de Nueva Gerona }\end{array}$ & $\mathrm{M}$ & - \\
\hline & MACN 39145 & $\begin{array}{l}\text { Cuba: Isla de la Juventud: } 14,7 \\
\text { mi al SSW de Nueva Gerona }\end{array}$ & M & - \\
\hline Rhaebo guttatus & INPA 15647 & Brazil: Amazonas & nd & - \\
\hline \multirow{3}{*}{ Schismaderma carens } & CENAI 6007 & nd & SA & - \\
\hline & USNM 153377 & Malawi: Rumphi: Rumph & nd & $\begin{array}{l}\mu \mathrm{CT} \text { (Morphosource ID } \\
23335 \text { ) }\end{array}$ \\
\hline & USNM 153380 & Malawi: Rumphi: Rumph & nd & $\begin{array}{l}\mu \mathrm{CT} \text { (Morphosource ID } \\
23336 \text { ) }\end{array}$ \\
\hline \multirow{2}{*}{ Sclerophrys mauritanica } & USNM 346809 & $\begin{array}{l}\text { Morocco: Tetouan: } 20 \mathrm{~km} \mathrm{SE} \\
\text { Larache }\end{array}$ & nd & $\begin{array}{l}\mu \mathrm{CT} \text { (Morphosource ID } \\
21854 \text { ) }\end{array}$ \\
\hline & USNM 346811 & $\begin{array}{l}\text { Morocco: Tetouan: } 20 \mathrm{~km} \mathrm{SE} \\
\text { Larache }\end{array}$ & nd & $\begin{array}{l}\mu \mathrm{CT} \text { (Morphosource ID } \\
21855 \text { ) }\end{array}$ \\
\hline Sclerophrys regularis & MZUSP 148117 & Guiné Bissau: Beli, Boé & M & - \\
\hline
\end{tabular}




\section{APPENDIX 5}

Assignation of the Species of Rhinella to Species Groups by Different Authors Assignations provided in the original description of the species are indicated with an asterisk $\left(^{*}\right)$.

\begin{tabular}{|c|c|c|}
\hline Species & Original description & Species group assigned \\
\hline R. abei & Baldissera et al., 2004 (as Bufo abei) & R. crucifer Group* \\
\hline R. achalensis & Cei, 1972b (as Bufo achalensis) & R. spinulosa Group* \\
\hline R. achavali & Maneyro et al., 2004 (as Bufo achavali) & R. marina Group ${ }^{*}$ \\
\hline R. acrolopha & Trueb, 1971 (as Rhamphophryne acrolopha) & R. acrolopha Group (Grant and Bolivar-G., 2014) \\
\hline R. acutirostris & Spix, 1824 (as Bufo acutirostris) & R. margaritifera Group (Hoogmoed, 1986) \\
\hline R. alata & Thominot, 1884 (as Bufo alatus) & R. margaritifera Group (Cei, 1972b) \\
\hline R. amabilis & Pramuk and Kadivar, 2003 (as Bufo amabilis) & R. spinulosa Group ${ }^{*}$ \\
\hline R. amboroensis & Harvey and Smith, 1993 (as Bufo amboroensis) & R. veraguensis Group* \\
\hline$R$. arborescandens & $\begin{array}{l}\text { Duellman and Schulte, } 1992 \\
\text { (as Bufo arborescandens) }\end{array}$ & R. veraguensis Group ${ }^{*}$ \\
\hline R. arenarum & Hensel, 1867 (as Bufo arenarum) & $\begin{array}{l}\text { R. marina Group (Martin, 1972b); R. arenarum } \\
\text { Group (Cei, 1980); R. marina Group (Duellman and } \\
\text { Schulte, 1992) }\end{array}$ \\
\hline$R$. arequipensis & Vellard, 1959 (as Bufo spinulosus arequipensis) & R. spinulosa Group ${ }^{\star}$ \\
\hline R. arunco & Molina, 1782 (as Rana arunco) & R. spinulosa Group (Martin, 1972b) \\
\hline R. atacamensis & Cei, 1962 (as Bufo spinulosus atacamensis) & R. spinulosa Group* \\
\hline R. azarai & Gallardo, 1965 (as Bufo granulosus azarai) & R. granulosa Group (Cei, 1972b) \\
\hline R. beebei & Gallardo, 1965 (as Bufo granulosus beebei) & R. granulosa Group (Cei, 1972b) \\
\hline R. bergi & Céspedez, 2000 (as Bufo bergi) & R. granulosa Group ${ }^{*}$ \\
\hline R. bernardoi & Sanabria et al., 2010 & R. granulosa Group ${ }^{*}$ \\
\hline R. casconi & Roberto et al., 2014 & R. crucifer Group* \\
\hline R. castaneotica & Caldwell, 1991 (as Bufo castaneoticus) & R. margaritifera Group ${ }^{*}$ \\
\hline R. centralis & Narvaes and Rodrigues, 2009 & R. granulosa Group ${ }^{*}$ \\
\hline R. ceratophrys & Boulenger, 1882 (as Bufo ceratophrys) & $\begin{array}{l}\text { R. margaritifera Group (Cei, 1972b); unnassigned } \\
\text { (Pramuk, 2006) }\end{array}$ \\
\hline R. cerradensis & Maciel et al., 2007 & R. marina Group ${ }^{*}$ \\
\hline R. chavin & Lehr et al., 2001 (as Bufo chavin) & $\begin{array}{l}\text { R. veraguensis Group }{ }^{*} \text { R. festae Group (Moravec et } \\
\text { al. 2014) }\end{array}$ \\
\hline R. chrysophora & $\begin{array}{l}\text { McCranie et al., } 1989 \\
\text { (as Atelophryniscus chrysophorus) }\end{array}$ & R. veraguensis Group (Pramuk and Lehr, 2005) \\
\hline R. cristinae & Vélez and Ruiz, 2002 (as Bufo cristinae) & $\begin{array}{l}\text { R. margaritifera Group (Pramuk 2006); unassigned } \\
\text { (Fouquet et al., 2007a) }\end{array}$ \\
\hline R. crucifer & Wied, 1821 (as Bufo crucifer) & R. crucifer Group (Martin, 1972b) \\
\hline R. dapsilis & Myers and Carvalho, 1945 (as Bufo dapsilis) & R. margaritifera Group* \\
\hline R. diptycha & Cope, 1862 (as Bufo diptychus) & $\begin{array}{l}\text { R. diptycha Group (Vellard, 1959); unassigned } \\
\text { (Duellman and Schulte, 1992); R. marina Group } \\
\text { (Lavilla and Brusquetti, 2018) }\end{array}$ \\
\hline R. dorbignyi & Duméril and Bibron, 1841 (as Bufo dorbignyi) & R. granulosa Group (Cei, 1972b; Martin, 1972b) \\
\hline R. fernandezae & Gallardo, 1957 (as Bufo granulosus fernandezae) & R. granulosa Group (Cei, 1972b; Martin, 1972b) \\
\hline
\end{tabular}


APPENDIX 5 continued

\begin{tabular}{|c|c|c|}
\hline Species & Original description & Species group assigned \\
\hline R. festae & Peracca, 1904 (as Atelopus festae) & $\begin{array}{l}\text { R. acrolopha Group (Grant and Bolivar-G.); R. festae } \\
\text { Group (Moravec et al., 2014) }\end{array}$ \\
\hline R. fissipes & Boulenger, 1903 (as Bufo fissipes) & $\begin{array}{l}\text { R. marina Group (Vellard, 1959); R. margaritifera Group } \\
\text { (Cei, 1972b), R. veraguensis Group (Hoogmoed, 1990) }\end{array}$ \\
\hline R. gallardoi & Carrizo, 1992 (as Bufo gallardoi) & R. veraguensis-margaritifera Group* \\
\hline R. gildae & Vaz-Silva et al., 2015 & R. margaritifera Group ${ }^{*}$ \\
\hline R. gnustae & Gallardo, 1967 (as Bufo gnustae) & Unassigned (Duellman and Schulte, 1992) \\
\hline R. granulosa & Spix, 1824 (as Bufo granulosus) & R. granulosa Group (Cei, 1972b) \\
\hline R. henseli & Lutz, 1934 (as Bufo crucifer henseli) & R. crucifer Group (Baldissera et al. 2004) \\
\hline R. hoogmoedi & Caramaschi and Pombal, 2006 & R. margaritifera Group* \\
\hline R. horribilis & Wiegmann, 1833 (as Bufo horribilis) & R. marina Group (Acevedo et al., 2016) \\
\hline R. humboldti & Gallardo, 1965 (as Bufo granulosus humboldti) & R. granulosa Group (Cei, 1972b; Martin, 1972b) \\
\hline R. icterica & Spix, 1824 (as Bufo ictericus) & R. marina Group (Martin, 1972b) \\
\hline R. inca & Stejneger, 1913 (as Bufo inca) & $\begin{array}{l}\text { R. margaritifera Group (Vellard, 1959); R. veraguensis } \\
\text { Group (Gallardo, 1961), R. margaritifera Group (Cei, } \\
\text { 1972b); R. veraguensis group (Hoogmoed, 1990) }\end{array}$ \\
\hline R. inopina & Vaz-Silva et al., 2012 & R. crucifer Group ${ }^{*}$ \\
\hline R. iserni & $\begin{array}{l}\text { Jiménez de la Espada, } 1875 \\
\text { (as Oxyrhynchus iserni) }\end{array}$ & $\begin{array}{l}\text { R. margaritifera Group (Hoogmoed, 1986); unas- } \\
\text { signed (Fouquet et al., 2007a) }\end{array}$ \\
\hline R. jimi & Stevaux, 2002 (as Bufo jimi) & R. marina Group* \\
\hline R. justinianoi & Harvey and Smith, 1994 (as Bufo justinianoi) & R. veraguensis Group ${ }^{*}$ \\
\hline R. leptoscelis & Boulenger, 1912 (as Bufo leptoscelis) & $\begin{array}{l}\text { R. margaritifera Group (Vellard, 1959); } R \text {. veraguensis } \\
\text { Group (Gallardo 1961); R. margaritifera Group (Cei, } \\
\text { 1972b); R. veraguensis Group (Hoogmoed, 1990) }\end{array}$ \\
\hline R. lescurei & Fouquet et al., 2007a & R. margaritifera Group ${ }^{*}$ \\
\hline R. lilyrodriguezae & Cusi et al., 2017 & R. festae Group ${ }^{\star}$ \\
\hline R. limensis & Werner, 1901 (as Bufo limensis) & R. spinulosa Group (Vellard, 1959) \\
\hline R. lindae & $\begin{array}{l}\text { Rivero and Castaño, } 1990 \\
\text { (as Rhamphophryne lindae) }\end{array}$ & R. acrolopha Group (Grant and Bolivar, 2014) \\
\hline R. macrorhina & Trueb, 1971 (as Rhamphophryne macrorhina) & $\begin{array}{l}\text { R. acrolopha Group (Grant and Bolivar-G., 2014); } R \text {. } \\
\text { festae Group (Moravec et al., 2014) }\end{array}$ \\
\hline R. magnussoni & Lima et al., 2007 & R. margaritifera Group $^{*}$ \\
\hline R. major & $\begin{array}{l}\text { Müller and Hellmich, } 1936 \\
\text { (as Bufo granulosus major) }\end{array}$ & R. granulosa Group (Martin, 1972b) \\
\hline R. manu & Chaparro et al., 2007 & R. festae Group (Moravec et al., 2014) \\
\hline R. margaritifera & Laurenti, 1768 (as Rana margaritifera) & R. margaritifera Group (Vellard, 1959) \\
\hline R. marina & Linnaeus, 1758 (as Rana marina) & R. marina Group (Vellard, 1959) \\
\hline R. martyi & Fouquet et al., 2007a & R. margaritifera Group* \\
\hline R. merianae & Gallardo, 1965 (as Bufo granulosus merianae) & R. granulosa Group (Cei, 1972b) \\
\hline R. mirandaribeiroi & $\begin{array}{l}\text { Gallardo, } 1965 \\
\text { (as Bufo granulosus mirandaribeiroi) }\end{array}$ & R. granulosa Group (Cei, 1972b) \\
\hline R. multiverrucosa & Lehr et al., 2005 (as Bufo multiverrucosus) & R. veraguensis Group ${ }^{*}$ \\
\hline
\end{tabular}


APPENDIX 5 continued

\begin{tabular}{|c|c|c|}
\hline Species & Original description & Species group assigned \\
\hline R. nattereri & Bokermann, 1967 (as Bufo granulosus nattereri) & R. granulosa Group (Cei, 1972b) \\
\hline R. nesiotes & Duellman and Toft, 1979 (as Bufo nesiotes) & $\begin{array}{l}\text { R. veraguensis Group }{ }^{*} \text { R. festae Group (Moravec et } \\
\text { al., 2014) }\end{array}$ \\
\hline R. nicefori & $\begin{array}{l}\text { Cochran and Goin, } 1970 \\
\text { (as Bufo rostratus nicefori) }\end{array}$ & R. acrolopha Group (Grant and Bolivar-G., 2014) \\
\hline R. ocellata & Günther, $1858 \mathrm{~b}$ (as Bufo ocellatus) & $\begin{array}{l}\text { R. margaritifera Group (Cei, 1972b); unassigned } \\
\text { Hoogmoed, 1990); R. margaritifera Group (Lavilla et } \\
\text { al., 2013) }\end{array}$ \\
\hline R. ornata & Spix, 1824 (as Bufo ornatus) & R. crucifer Group ${ }^{*}$ \\
\hline R. paraguas & Grant and Bolívar-G., 2014 & R. acrolopha Group ${ }^{*}$ \\
\hline R. paraguayensis & Ávila et al., 2010 & R. margaritifera Group ${ }^{*}$ \\
\hline R. poeppigii & Tschudi, 1845 (as Bufo poeppigii) & R. marina Group (Vellard, 1959) \\
\hline R. proboscidea & Spix, 1824 (as Bufo proboscideus) & R. margaritifera Group (Hoogmoed, 1986) \\
\hline R. pygmaea & Myers and Carvalho, 1952 (as Bufo pygmaeus) & R. granulosa Group (Cei, 1972b) \\
\hline R. quechua & Gallardo, 1961 (as Bufo quechua) & $\begin{array}{l}\text { R. veraguensis Group }{ }^{*} ; \text { R. margaritifera Group (Cei, } \\
\text { 1972b); R. veraguensis Group (Hoogmoed, 1990) }\end{array}$ \\
\hline R. roqueana & Melin, 1941 (as Bufo typhonius roqueanus) & R. margaritifera Group (Hoogmoed, 1986) \\
\hline R. rostrata & Noble, 1920 (as Bufo rostratus) & $\begin{array}{l}\text { R. acrolopha Group (Grant and Bolivar-G., 2014); } R \text {. } \\
\text { festae Group (Moravec et al., 2014) }\end{array}$ \\
\hline R. rubescens & Lutz, 1925 (as Bufo rubescens) & $\begin{array}{l}\text { R. marina Group (Cei, 1972b); R. arenarum Group } \\
(\text { Cei, 1980) }\end{array}$ \\
\hline R. rubropunctata & Guichenot, 1848 (as Bufo rubropunctatus) & R. spinulosa Group (Cei, 1972b) \\
\hline R. ruizi & Grant, 2000 (as Rhamphophryne ruizi) & R. acrolopha (Grant and Bolivar-G., 2014) \\
\hline R. rumbolli & Carrizo, 1992 (as Bufo rumbolli) & R. veraguensis Group ${ }^{*}$ \\
\hline R. scitula & $\begin{array}{l}\text { Caramaschi and Niemeyer, } 2003 \\
\text { (as Bufo scitulus) }\end{array}$ & R. margaritifera Group ${ }^{*}$ \\
\hline R. sclerocephala & $\begin{array}{l}\text { Mijares and Arends, } 2001 \\
\text { (as Bufo sclerocephalus) }\end{array}$ & R. margaritifera Group ${ }^{*}$ \\
\hline R. sebbeni & Vaz-Silva et al., 2015 & R. margaritifera Group ${ }^{*}$ \\
\hline R. spinulosa & Wiegmann, 1834 (as Bufo spinulosus) & R. spinulosa Group (Vellard, 1959) \\
\hline R. stanlaii & Lötters and Köhler, 2000 (as Bufo stanlaii) & R. margaritifera Group* \\
\hline R. sternosignata & Günther, 1858b (as Bufo sternosignatus) & $\begin{array}{l}\text { R. margaritifera Group (Cei, 1972b); unassigned } \\
\text { (Hoogmoed, 1990); R. margaritifera Group } \\
\text { (Duellman and Schulte, 1992); unassigned (Fouquet } \\
\text { et al., 2007a) }\end{array}$ \\
\hline R. tacana & Padial et al., 2006 (as Chaunus tacana) & $R$. veraguensis Group ${ }^{*}$ \\
\hline R. tenrec & $\begin{array}{l}\text { Lynch and Renjifo, } 1990 \\
\text { (as Rhamphophryne tenrec) }\end{array}$ & R. acrolopha (Grant and Bolivar-G., 2014) \\
\hline R. truebae & $\begin{array}{l}\text { Lynch and Renjifo, } 1990 \\
\text { (as Rhamphophryne truebae) }\end{array}$ & R. acrolopha (Grant and Bolivar-G., 2014) \\
\hline R. vellardi & Leviton and Duellman, 1978 (as Bufo vellardi) & R. spinulosa Group* \\
\hline R. veraguensis & Schmidt, 1857 (as Bufo veraguensis) & $\begin{array}{l}\text { R. margaritifera Group (Vellard, 1959); } R \text {. veraguensis } \\
\text { Group (Gallardo, 1961); R. margaritifera Group (Cei, } \\
\text { 1972b); R. veraguensis Group (Hoogmoed, 1990) }\end{array}$ \\
\hline
\end{tabular}


APPENDIX 5 continued

\begin{tabular}{lll}
\hline \hline Species & Original description & Species group assigned \\
\hline R. veredas & Brandão et al., 2007 (as Chaunus veredas) & R. marina Group* \\
\hline R. yanachaga & Lehr et al., 2007 & $\begin{array}{l}\text { R. veraguensis Group } \\
\text { al., } \text { R. fostae Group (Moravec et }\end{array}$ \\
\hline R. yunga & Moravec et al., 2014 & R. margaritifera Group* \\
\hline
\end{tabular}

\section{NOTE ADDED IN PROOF}

Two new species of the Rhinella margaritifera Group were described while this publication was in the proofing stage: $R$. parecis Ávila et al., 2020, from Brazil and $R$. exostosica Ferrão et al., 2020, from Bolivia, Brazil, and Peru. The inclusion of the available 16S sequence (KDQF01003635, voucher specimen MTR 25730) of $R$. parecis in our TE dataset and the parsimony analysis in TNT found this specimen as the sister taxon to Rhinella sp. 12 (although with low support; JAF $=51 \%$ ). Rhinella exostosica corresponds to Rhinella sp. 14, as our study and that of Ferrão et al. (2020) included some GenBank sequences in common from three terminals (KU 215145-6 and NMP6V 74915).

\section{REFERENCES:}

Ávila, R.W., et al. 2020. A new species of the Rhinella margaritifera (Laurenti 1768) species group (Anura, Bufonidae) from southern Brazilian Amazonia. Zootaxa 4868: 368-388.

Ferrão, M., A.P. Lima, S.R. Ron, S.P.L. dos Santos, and J. Hanken. 2020. New species of leaf-litter toad of the Rhinella margaritifera species group (Anura: Bufonidae) from Amazonia. Copeia 108: 967-986. 



\title{
SCientific Publications of the American Museum of Natural History
}

AMERICAN MUSEUM NOVITATES

BULletin of the American Museum of Natural History

Anthropological papers of the american Museum of Natural History

\author{
Publications Committee \\ ROBERT S. VOSS, CHAIR \\ BOARD OF EDITORS \\ Jin MENG, PALEONTOLOGY \\ LORENZO PRENDINI, INVERTEBRATE ZOOLOGY \\ ROBERT S. VOSS, VERTEBRATE ZOOLOGY \\ Peter M. Whiteley, Anthropology \\ MANAGING EDITOR \\ MARY KNIGHT
}

Submission procedures can be found at http://research.amnh.org/scipubs

All issues of Novitates and Bulletin are available on the web (http://digitallibrary.amnh.

org/dspace). Order printed copies on the web from:

http://shop.amnh.org/a701/shop-by-category/books/scientific-publications.html

or via standard mail from:

American Museum of Natural History-Scientific Publications

Central Park West at 79th Street

New York, NY 10024

(2) This paper meets the requirements of ANSI/NISO Z39.48-1992 (permanence of paper).

ON the COVER: Representatives of the PHYLOGENETIC DiVERSity OF RHINELLA.OUTER RING,CLOCKWISE FROMTOP:R. LINDAE (PHOTO BYM.RADA), R. RUBESCENS (C.F.B. HADDAD), R. MANU (I. DE LA RIVA), R. ALTIPERUVIANA (B.L. Blotto), R. lilyrodriguezaE (P. VENEGAS), R. PARAGUAS (T. GRANT), R. ATACAMENSIS (V. VALDÉS), R. HUMBOLDTI (I. DE LA RIVA), R. CASTANEOTICA (T. GRANT), R. orNAtA (M. TEXEIRA, JR.), R. DORBIGNYI (F. KOLENC), AND R. DAPSILIS (S. RON). INNER RING, CLOCKWISE FROM TOP: R. CRUCIFER (C.F.B. HAdDAD), R. sternosignata (C. BARrio-Amorós), R. quechua (A. MuÑoz), R. marina (J. MORAVEC), AND R. VERAGUENSIS (I. DE LA Riva). 